\title{
MELT RATE IMPROVEMENT FOR DWPF MB3:
}

\section{Frit Development and Model Assessment (U)}

\author{
D.K. Peeler \\ T.H. Lorier \\ D.F. Bickford \\ D.C. Witt
}

T.B. Edwards

K.G. Brown

I.A. Reamer

R.J. Workman

Westinghouse Savannah River Company

Savannah River Technology Center

Aiken, SC 29808

\section{J.D. Vienna}

Pacific Northwest National Laboratory

Richland, WA

TTP\#: SR-1-6-WT-31

TTR\#: HLW/DWPF/TTR-00-0044

Westinghouse Savannah River Company

Savannah River Technology Center Aiken, SC 29808

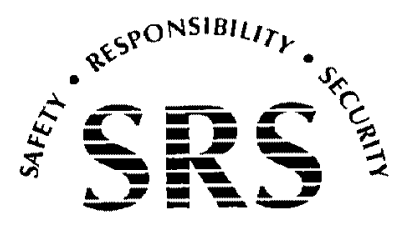


This document was prepared in conjunction with work accomplished under Contract No.

DE-AC09-96SR18500 with the U.S. Department of Energy.

\section{DISCLAIMER}

This report was prepared as an account of work sponsored by an agency of the United States Government. Neither the United States Government nor any agency thereof, nor any of their employees, makes any warranty, express or implied, or assumes any legal liability or responsibility for the accuracy, completeness, or usefulness of any information, apparatus, product or process disclosed, or represents that its use would not infringe privately owned rights. Reference herein to any specific commercial product, process or service by trade name, trademark, manufacturer, or otherwise does not necessarily constitute or imply its endorsement, recommendation, or favoring by the United States Government or any agency thereof. The views and opinions of authors expressed herein do not necessarily state or reflect those of the United States Government or any agency thereof.

This report has been reproduced directly from the best available copy.

Available for sale to the public, in paper, from: U.S. Department of Commerce, National Technical Information Service, 5285 Port Royal Road, Springfield, VA 22161, phone: (800)

553-6847, fax: (703) 605-6900, email: orders@ ntis.fedworld.gov online ordering: http://www.ntis.gov/ordering.htm

Available electronically at http://www.doe.gov/bridge

Available for a processing fee to U.S. Department of Energy and its contractors, in paper, from: U.S. Department of Energy, Office of Scientific and Technical Information, P.O. Box 62, Oak Ridge, TN 37831-0062, phone: (865 ) 576-8401, fax: (865) 576-5728, email: reports@adonis.osti.gov 


\title{
IMPROVE MELT RATE FOR DWPF MB3:
}

\section{FRIT DEVELOPMENT AND MODEL ASSESSMENT (U)}

\author{
D.K. Peeler \\ T.B. Edwards \\ T.H. Lorier \\ K.G. Brown \\ D.F. Bickford \\ I.A. Reamer \\ D.C. Witt \\ R.J. Workman \\ Westinghouse Savannah River Company \\ Savannah River Technology Center \\ Aiken, SC
}

\section{J.D. Vienna \\ Pacific Northwest National Laboratory \\ Richland, WA}

This report was prepared by Westinghouse Savannah River Company (WSRC) for the United States Department of Energy under Contract No. DE-AC09-96SR18500 and is an account of work performed under that contract. 


\begin{tabular}{|c|c|c|c|}
\hline Task Title: Improve Melt Rate & & $\begin{array}{l}\text { TTR Number: } \\
\text { HLW/DWPF/TTR- } \\
\text { 00-0044, Rev. } 0\end{array}$ & $\begin{array}{l}\text { TTR Date: } \\
10 / 24 / 00\end{array}$ \\
\hline $\begin{array}{l}\text { Task Leader: } \\
\text { D.K. Peeler }\end{array}$ & signaura Ledel & $\begin{array}{l}\text { Organization: } \\
\text { ITS }\end{array}$ & $\begin{array}{l}\text { Date: } \\
5-8-01\end{array}$ \\
\hline $\begin{array}{l}\text { Task Leader: } \\
\text { T.H. Lorier }\end{array}$ & Signature: & $\begin{array}{l}\text { Organization: } \\
\text { ITS } \\
\end{array}$ & $\begin{array}{l}\text { Date: } \\
5-9-01\end{array}$ \\
\hline $\begin{array}{l}\text { Task Leader: } \\
\text { J.D. Vienna }\end{array}$ & sighatury & $\begin{array}{l}\text { Organization: } \\
\text { PNNL }\end{array}$ & Date: $15 / 01$ \\
\hline $\begin{array}{l}\text { Task Leader: } \\
\text { T.B. Edwards }\end{array}$ & gendom & $\begin{array}{l}\text { Organization: } \\
\text { SCS }\end{array}$ & Date: $-9-0$ \\
\hline $\begin{array}{l}\text { Technical Reviewer: } \\
\text { C.M. Jantzen }\end{array}$ & Signature: M Lantien & $\begin{array}{l}\text { Organization: } \\
\text { ITS }\end{array}$ & $\begin{array}{l}\text { Date: } \\
5-10-01\end{array}$ \\
\hline $\begin{array}{l}\text { Technical Reviewer: } \\
\text { A.D. Cozzi }\end{array}$ & Signature: Col 8 & $\begin{array}{l}\text { Organization: } \\
\text { ITS }\end{array}$ & $\begin{array}{l}\text { Date: } \\
5-9-01\end{array}$ \\
\hline $\begin{array}{l}\text { Level } 3 \text { Manager: } \\
\text { E.W. Holtzscheiter }\end{array}$ & Signaturetraherts & $\begin{array}{l}\text { Organization: } \\
\text { ITS } \\
\end{array}$ & \begin{tabular}{|l} 
Date: \\
$5-10-01$
\end{tabular} \\
\hline $\begin{array}{l}\text { Level } 4 \text { Manager: } \\
\text { S.L. Marra }\end{array}$ & Signature: & $\begin{array}{l}\text { Organization: } \\
\text { ITS }\end{array}$ & Date: \\
\hline
\end{tabular}




\section{GLOSSARY}

$\eta$

ADS

AES

ARM

ASTM

bc

CELS

clc

CVS

DTA

DWPF

EA

$\mathrm{g} / \mathrm{L}$

ICP

HLW

LM

MB2

MB3

NL

PAR

PCCS

PCT

PF

PHA

PNNL

ppm

SME

SRAT

SRTC

SRTC-ML

TGA viscosity

Analytical Development Section

atomic emission spectroscopy

Approved Reference Material

American Society for Testing and Materials

bias corrected

Corning Engineering Laboratory Services

centerline canister cooled

composition variation study

differential thermal analysis

Defense Waste Processing Facility

Environmental Assessment

grams per liter

inductively coupled plasma

high-level waste

lithium metaborate

macrobatch 2

macrobatch 3

normalized leachate

Property Acceptability Region

Product Composition Control System

product consistency test

peroxide fusion

precipitate hydrolysis aqueous

Pacific Northwest National Laboratory

parts per million

slurry mix evaporator

slurry receipt and adjustment tank

Savannah River Technology Center

Savannah River Technology Center - Mobile Laboratory

thermogravimetric analysis 
Immobilization Technology Section

Savannah River Technology Center

Rev. 0

Westinghouse Savannah River Company

TFA Tanks Focus Area

THERMO $^{\mathrm{TM}}$ Thermodynamic Hydration Energy Reaction Model

$\mathrm{T}_{\mathrm{L}} \quad$ liquidus temperature

TTR Task Technical Request

$\mathrm{U}_{\text {std }} \quad$ uranium-containing glass

WAPS Waste Acceptance Product Specifications

WCP Wasteform Compliance Plan

WL waste loading

WSRC Westinghouse Savannah River Company 


\section{ACKNOWLEDGMENTS}

The authors would like to acknowledge Alex Cozzi, Carol Jantzen, Dan Lambert, Mike Stone (Savannah River Technology Center) and Pavel Hrma (Pacific Northwest National Laboratory) for their technical guidance and reviews, and William Holtzscheiter (Tanks Focus Area Technical Integration Manager for Immobilization) and Sharon Marra for management and guidance. This study was co-funded by the Department of Energy's Office of Science and Technology through the Tanks Focus Area and through the Defense Waste Processing Facility. Westinghouse Savannah River Company is operated to the U.S. Department of Energy under Contract No. DE-AC09-96SR18500. 
Immobilization Technology Section

Savannah River Technology Center

Rev. 0

Westinghouse Savannah River Company

This page intentionally left blank. 


\section{CONTENTS}

GLOSSARY

ACKNOWLEDGEMENTS

LIST OF FIGURES

LIST OF TABLES

1.0 INTRODUCTION

2.0 BACKGROUND: PREVIOUS WORK ON FRIT OPTIMIZATION FOR DWPF

3.0 OBJECTIVE

4.0 MB3 WASTE COMPOSITION

5.0 Frit DeVELOPMENT AND Model ASSESSMENT ACTIVITIES

5.1 Frit Development

5.2 PCCS Model Assessment of Candidate Frits

5.2.1 Nominal and Underwashed Scenarios at Maximum Waste Loading

5.2.2 Nominal and Underwashed Scenarios at a Fixed Waste Loading

6.0 EXPERIMENTAL

6.1 Glass Fabrication

6.2 Property Measurements

6.2.1 Chemical Composition Analysis

6.2.2 Durability

6.2.3 Viscosity

7.0 RESULTS AND DISCUSSION

7.1 Chemical Composition Analysis

7.2 Durability

7.2.1 PCT Results for the Glasses Representing the Nominally Washed Sludge

7.2.2 PCT Results for the Glasses Representing the Underwashed Sludge

7.2.3 PCT Results versus Model Predictions

7.2.4 Quenched Versus Centerline Cooled PCTs

7.3 Viscosity

8.0 ISSUES AND IMPACTS OF SME ACCEPTABILITY

9.0 SUMMARY

10.0 REFERENCES

APPENDICES 


\section{LIST OF FIGURES}

Figure 1. Pair-Wise Plots (Scatter Plot Matrix) of Calculated Frit Compositions (in weight fraction).

Figure 2. Quenched versus CLC PCTs for Nominal Case.

Figure 3. Quenched versus CLC PCTs for Underwashed Case.

Figure 4. XRD Results of the MB3N313q.

Figure 5. XRD Results of the MB3N313clc.

Figure 6. XRD Results of the MB3U313q.

Figure 7. XRD Results of the MB3U313clc.

Figure 8. Schematic of $\Delta \mathrm{G}_{\mathrm{P}}$ Versus Log NL [B] (g/L) for Various MB3 Glasses. 


\section{LIST OF TABLES}

Table 1. Oxide Sludge Concentrations (wt\%) of an Equal Blend of Tank 8 and Tank 40 (nominal, underwashed, and overwashed scenarios).

Table 2. Composition (wt\%) of MB3 Sludge Used for Initial Model Assessments and Frit Development Activities.

Table 3. First-Order Coefficients for Selected Glass Properties.

Table 4. Constraints Used in this Study on Predicted Glass Properties.

Table 5. Frit Compositions (wt\%) and Resultant Glass Property Predictions.

Table 6. Target and Measured Composition (wt\%) of MB3N320q at $25.5 \mathrm{wt} \%$ Sludge Loading.

Table 7. Comparison of Predicted and Measured Property Values for MB3N320q at $25.5 \mathrm{wt} \%$ Sludge Loading.

Table 8. Frit Composition (wt\%) of Existing Frits and Those Developed to Supplement the Existing Database.

Table 9. Candidate Frit Compositions and Estimated Properties at the Maximum Allowable Waste Loading (per PCCS model predictions) for Nominal and Underwashed MB3 Sludge.

Table 10. Candidate Frit Compositions and Estimated Properties at a Fixed Waste Loading (25.5 wt\%) (per PCCS model predictions) for Nominal and Underwashed MB3 Sludge.

Table 11. PAR Limits for Various Properties.

Table 12. Targeted Oxide Compositions (in weight percents, wt\%'s) of the MR Glasses.

Table 13. Average Measurements by Analytical Block for Glass Standards.

Table 14. Measured, Measured Bias-Corrected (bc), and targeted compositions for the MB3 Study Glasses.

Table 15. Average Measurements of Standard Solution by Analytical Block - PCTs for Glasses from Nominally Washed Sludge.

Table 16. Normalized PCTs before Screening for Solution-Weight Problems - Nominally Washed Sludge.

Table 17. Average Measurements of Standard Solution by Analytical Block - PCTs for Glasses from Underwashed Sludge.

Table 18. Average Leachate Concentrations from Screened and Unscreened PCTs.

Table 19. Measured and Predicted $\eta_{1150^{\circ} \mathrm{C}}$ (in Poise) of Select MB3 Glasses.

Table 20. Normalized Release for Glasses Produced with MB3 (Nominal and Underwashed Sludge). 


\section{APPENDICES}

Appendix A: Frit and Glass Nomenclature

Appendix B: Analytical Plan for Measuring Chemical Compositions (SRT-SCS-2001-00008)

Appendix C: Analytical Plan for Measuring PCT Solutions: Nominal MB3 Sludge (SRT-SCS-2001-00009)

Appendix D: Analytical Plan for Measuring PCT Solutions: Underwashed MB3 Sludge (SRT-SCS-2001-00011)

Appendix E: Tables of Chemical Composition Measurements, Tables of PCT Measurements, and Exhibits of Statistical Analyses 


\section{EXECUTIVE SUMMARY}

The objective of this research was to enhance the basic understanding of the role of glass batch chemistry (more specifically via control of frit composition) on the overall melting process for Macrobatch 3 (MB3). The overall strategy for the frit development activities was to explore frit compositional regions which challenged "acceptable" predicted property behavior. Once major frit components were identified, ranges were established to challenge current model predictions in an attempt to maximize melt rate. A series of frit compositions were developed not only to maintain the projected operational window (25$30 \%$ sludge oxide waste loading) relative to the Frit 200 baseline but to also increase melt rate. The intent of this effort was to explore compositional extremes in frit space to bound the effect on melt rate for MB3. While exploring these compositional limits, model assessments were made but not necessarily used to limit the final compositional envelope.

The decision or technical basis as to whether a candidate frit will improve melt rate relative to Frit 200 can not be made based on the model assessments or the limited data discussed in this report. However, the information presented in this report does provide input into the selection process for those glasses that have been shown to have an improved melt rate relative to the current Frit 200 baseline.

Glasses were fabricated using selected candidate frit compositions at a fixed target waste loading and various properties were measured. An important objective of this study was to investigate the potential impact on glass durability (as defined by the Product Consistency Test (PCT)) due to cooling rate. The data indicated no statistically significant difference between the quenched and centerline cooled PCTs for either the nominally washed or the underwashed MB3 sludge cases. The measured PCT data indicates that glasses produced from either the nominal or underwashed sludge (for both thermal heat treatments) are $<2 \mathrm{~g} / \mathrm{L}$ (for all reportable elements); these are still well below that of the Environmental Assessment (EA) glass.

Based on an assessment of durability, the majority of the frits developed satisfy the current slurry mix evaporator (SME) acceptability criteria (i.e., $\Delta \mathrm{G}_{\mathrm{P}}$ limit) and lie within the $95 \%$ prediction confidence interval indicating that the PCTs are well predicted by the current model. Selection of a frit in this category (i.e., SME acceptance for durability is passed and the model predicts well) lowers the additional data needs prior to implementation in the Defense Waste Processing Facility (DWPF). However, this path may result in a frit that does not optimize melt rate for MB3. 
Technical issues have been identified relative to SME acceptability issues for one candidate frit composition. Although the current durability model does not predict an acceptable release, the measured PCT data for glasses produced using this frit indicate that all elemental releases are less than $2 \mathrm{~g} / \mathrm{L}$.

For those glasses "failing" the current SME acceptability criteria for durability, alternative solutions have been proposed. Alternative pathways proposed include (but are not limited to): (i) developing nonparametric models over the composition regions, and/or (ii) refining the current DWPF durability model for this new composition region. Resolution of these alternative pathways is beyond the scope of this document and the supporting data.

It should be noted that the current task is focused specifically on improving melt rate for MB3. Although the "systems approach" will be utilized to the extent possible, this task does not attempt to optimize a frit for all "sludge-only" waste processing as projected by the current High Level Waste (HLW) System Plan. Therefore, the use of candidate frits to improve melt rate for MB3 may not be warranted for future sludge-only macrobatches.

The selection or recommendation of an alternative frit will ultimately be influenced by the relative increase in melt rate that one frit has over the current baseline, the acceptable risk level, and/or budget and schedule restrictions. The level of risk that is set must be balanced by the potential gains in terms of melter throughput. 


\subsection{INTRODUCTION}

Glass melting is a complex process that involves a number of reactions and transformations.

Consequently, it is necessary to identify the relevant processing properties for cold cap melting and possible laboratory test methods to evaluate these pertinent processing properties. Kim and Hrma (1994) suggested that developing appropriate laboratory test methods to assess melt rate should be preceded by understanding the basic processes involved in cold cap melting. The effects of various melter operating conditions and physical/chemical properties of the feed on the melting rate need to be clearly established. Because of the complexities involved, several analytical techniques should be used to characterize the local processes leading from batch to homogeneous glass and to link the bulk melt to the reaction at the interfaces.

The objective of this research was to enhance the basic understanding of the role of glass batch chemistry (more specifically via control of frit composition) on the overall melting process for Macrobatch 3 (MB3) (sludge-only processing). Through control of batch chemistry, cold cap reactions can be altered which may result in higher melter throughput. For melt rate limited systems, a small increase in melting efficiency translates into substantial savings by reducing operational costs without compromising product quality.

Although the primary focus was to improve melt rate for a specific macrobatch, a "systems approach" (Jantzen 1986) will be utilized to ensure other criteria important to glass production in DWPF will not be ignored. That is, any proposed frit composition change will be assessed using the current Thermodynamic Hydration Energy Reaction Model (THERMO ${ }^{\mathrm{TM}}$ ) predictions (Jantzen et al. 1995) in terms of the established Slurry Mix Evaporator (SME) acceptability criteria (Brown and Edwards 1995, Brown and Postles 1996). Potential chemical processing changes (see Lambert et al. 2001) must be tempered by a thorough assessment of related safety issues. If the systems approach or a thorough assessment of safety related issues are not utilized in an attempt to improve melt rate, one could make an off-specification glass faster or compromise melter processing and/or the safety basis.

Regardless of whether a potential frit composition change is acceptable or not in terms of model predictions, it is recommended that prior to implementation in the Defense Waste Processing Facility (DWPF) all properties be assessed to ensure that the proposed compositional changes do not invalidate current model predictions (e.g., the proposed glass composition is still within the compositional envelope over which the models were developed). It should be noted that frit development activities were directed 
solely toward MB3 and the use of a specific frit composition with another sludge batch may not be warranted.

This report does not address potential chemical processing changes (e.g., redox adjustments and/or acid addition strategies) and the downstream impacts they may have on process and product properties. Although the joint DWPF / Tanks Focus Area (TFA) program is evaluating both potential frit and/or redox/acid additions changes to enhance melt rate, this report focuses solely on compositional changes to the frit. Stone and Josephs (2001) provided a detailed discussion of the redox / acid addition strategy changes on enhancing melt rate.

Reliable recommendations for compositional and/or chemical processing changes will be possible if the mechanisms leading to foam are understood. Mechanisms of interest include the reaction pathway or kinetics of the batch-to-glass conversion process, off-gas behavior as a function of redox and/or decomposition reactions, and the temperature - viscosity $(\eta)$ relationships for both the glass and frit (Peeler et al. 2001). A detailed knowledge of these fundamental parameters will be necessary to determine the optimum chemical-processing or fit-composition changes needed. This being the case, it would be desirable to focus on one of the parameters leaving the other "fixed" and hope to bound the effect. In the event that both frit compositional and chemical process changes are independently identified to improve melt rate, these effects may not be additive. Therefore, prior to recommending a coupled change, the effects must be jointly evaluated. This assumes that the test methodology or suite of tests being used to assess melt rate directly translates to a full scale system.

As previously mentioned, because of the complexities involved, several analytical techniques should be used in an effort to gain a fundamental understanding of the mechanisms leading to a high melt rate. It would be ideal to have a single, inexpensive short-term test that could provide a direct measure of melt rate for the full-scale system. Unfortunately, such a test does not exist. Previous studies have utilized a specific test or suite of tests to assess melt rate for various glass systems. These included (but were not limited to): isothermal tests to assess melting behavior as a function of time and temperature, batch-free time studies, gradient furnace studies to identify the major processes occurring during the batch-to-glass conversion, quartz crucible studies to assess the propensity for batch expansion and to characterize offgas generated, DTA/TGA analysis of reaction pathways, evaluation of cold cap samples from melters, and melt rate furnace tests where unidirectional heat is applied to simulate heat transfer from the molten glass pool. 
This program utilized the available analytical tools within the allocated funds to develop a sound technical basis (minimizing risk to the extent possible) for the recommended change to the current baseline DWPF operations. Test protocols and methodology established through the recent work by Stone and Lambert (2000) for MB2 form the foundation used to assess melt rate for MB3. The primary tools to be utilized include: laboratory-scale isothermal tests and melt rate furnace tests. Additional tests and/or analytical tools will be utilized as warranted. Lambert et al. (2001) discussed additional tests that should be performed prior to implementation of a frit or chemical processing change in DWPF (e.g., slurry-fed melt rate and mini-melter tests).

This report discusses the primary assessment of potential frit changes on various properties via model predictions. It should be noted that models to directly assess melt rate are not available (or do not exist). However, the "systems approach" concept (Jantzen 1986) will be applied to ensure that although a candidate frit may improve melt rate other properties (e.g., durability, liquidus temperature $\left(\mathrm{T}_{\mathrm{L}}\right)$, and $\eta$ ) will not be compromised. Property - composition models will be used to assess the impact of a range of frit compositions on various properties. A limited number of tests will be performed on critical properties of interest in an effort to confirm model predictions and/or reduce technical risks.

This report is one in a series of reports that provide details on the MB3 melt rate improvement task. Lambert et al. (2001) provided the recommendations resulting from this integrated effort. The focus of the current report is on the joint Savannah River Technology Center (SRTC) / Pacific Northwest National Laboratory (PNNL) frit development and model assessment activities leading to candidate frit compositions to improve melt rate for MB3. Section 2 of this report provides a brief discussion of previous frit development activities for the Defense Waste Processing Facility (DWPF). Section 3 describes the objectives of the overall program to improve melt rate for MB3. The MB3 waste composition is discussed in Section 4. Section 5 presents the SRTC / PNNL frit development and model assessment activities. In Sections 6 and 7 we describe a limited number of tests that were performed on critical properties of interest (durability and $\eta$ ) in an effort to validate model predictions and reduce technical risks. Section 8 addresses technical issues regarding SME acceptability for candidate Frit compositions and provides input to the selection process for those glasses that have been shown to have an improved melt rate relative to the current Frit 200 baseline. Section 9 presents a summary and conclusions regarding the frit development and model assessment activities. This work is being performed in response to Technical Task Request (TTR) \#HLW/DWPF/TTR-00-0044, DWPF Macrobatch 3 Melt Rate Study. 


\subsection{Background: Previous Work On Frit Optimization for DWPF}

Soper et al. (1983) defined an "optimum" sludge-only frit as "one which produced waste glass with leachability as low as possible, with a maximum viscosity at $1150^{\circ} \mathrm{C}$ as near $15 \mathrm{~N}-\mathrm{s} / \mathrm{m}^{2}$ (or Pa-s) as possible, with a liquidus temperature as low as possible and with a coefficient of thermal expansion as low as possible." Through a statistically designed study and after only 25 trials, a frit meeting this definition was found in spite of the fact that 8 chemical components were evaluated. Frit 165 was found to be superior to other potential frit candidates (including Frit 131) for sludge-only processing.

Jantzen (1988) mathematically developed a family of glasses for DWPF for both coupled-feed (waste sludge plus precipitate hydrolysis aqueous (PHA) product) based on Soper's work. The glasses were formulated to meet specific durability and processing $\left(T_{L}\right.$ and $\left.\eta\right)$ criteria. One such criterion was that of melt temperature which was limited to $1150^{\circ} \mathrm{C}$ by volatilization of radionuclides (e.g., cesium and ruthenium) and the need to minimize corrosion / erosion of materials of construction. Therefore, frits were developed to dissolve the waste in the glass at this temperature while maintaining a high resistance to aqueous attack. Based on processing, fabrication, and durability considerations, Frit 202 (the coupledfeed frit formulation with the lowest alkali content) was recommended for initial DWPF coupledoperations.

It should be noted that the current task focuses specifically on MB3 and improving melt rate. Although the "systems approach" (Jantzen 1986) will be utilized to the extent possible, this task does not attempt to optimize a frit for all "sludge-only" waste processing as projected by the current HLW System Plan (HLW 2000). Therefore, the use of candidate frits to improve melt rate for MB3 may not be warranted for future sludge-only macrobatches.

Although this research is focused on increasing melt rate for MB3, the incentive stems from the following issues:

1. Interim Frit: A frit designed for coupled operation (e.g., the Frit 200 baseline) was being used as an "interim frit" for sludge-only operations until a decision was made on alternative salt processing.

2. Poor Melt Rate: Since the additional alkali and boron from salt processing is now questionable and will be delayed for several years, the DWPF melt rate was poor because of this flux deficiency. 
3. High $\mathbf{A l}_{2} \mathbf{O}_{3}$ Sludge: $\mathrm{MB} 3$ is a high-alumina-containing sludge that, if used with the interim frit, would make the attainment of melt rate even worse, e.g., high-iron-containing sludges could be used with the interim frit, but high-alumina sludge cannot.

4. Less Alkali Removal: Removal of alkali from sludge in the tank farm was proving problematic, and there was a desire to process the sludge less, e.g., wash less alkali out of the sludge, than in previous sludge-only flowsheets developed in the 1980-1990 timeframe.

5. Multiple Frits: Due to limited space in the tank farm for blending sludge types, e.g., high Al with high $\mathrm{Fe}$, the strategy of having one frit that can accommodate every waste type is being re-examined in favor of a strategy to optimize a frit for each type of sludge.

\subsection{OBJECTIVE}

The objective of this research was to enhance the basic understanding of the role of glass batch chemistry (more specifically via control of frit composition) and/or changes in acid addition strategies on the overall melting process for MB3 (sludge-only processing). Through control of batch chemistry, cold cap reactions can be altered resulting in higher melter throughput. For melt rate limited systems, a small increase in melting efficiency translates into substantial savings by reducing operational costs without compromising the quality of the final waste form or product.

\subsection{MB3 WASTE COMPOSITION}

According to the HLW System Plan (HLW 2000), the next sludge batch to be processed will be sludge batch 2 (referred to as MB3). Macrobatch 3 is assumed to be an equal blend of Tank 8 and Tank 40 . Harbour et al. (2000) have completed the variability study, which is required prior to receiving this sludge in DWPF. The batch will also be qualified by process simulation with actual waste in the SRTC high level caves. Elder (2000) provided the target sludge compositions obtained from the HLW Database (Hester 1996). Elder indicated that the compositions derived compared favorably with samples taken from streams with similar histories.

Elder (2000) noted that the extent of sludge washing was somewhat uncertain which could result in variation of sodium and aluminum (to a lesser extent given aluminum dissolution issues) concentrations in the sludge feed to the melter. This potential unknown was accounted for in the fact that nominal, underwashed, and overwashed sludge scenarios were calculated. 
Harbour et al. (2000) and Edwards (2000) also utilized Elder's sludge estimates as the basis for the MB3 variability study. The variability study evaluated two potential scenarios for processing MB3: (1) Tank 40 only and (2) a blended sludge consisting of equal parts (on an oxide wt\% basis) of Tank 8 and Tank 40 (representing a transfer of the complete contents of Tank 8 into Tank 40). 1 For the current MB3 melt rate study, only the blended sludge option will be considered. The oxide concentrations for this option (i.e., sludge produced from an equal blend of Tank 8 / Tank 40) are given in Table 1.

${ }^{1}$ Since Elder's report was issued, Tank 8 has been transferred into Tank 40. 
Table 1. Oxide Sludge Concentrations (wt\%) of an Equal Blend of Tank 8 and Tank 40.

\begin{tabular}{|c|c|c|c|}
\hline \multirow[b]{2}{*}{ Oxide } & \multicolumn{3}{|c|}{ Tank 8/40 Blend } \\
\hline & Underwashed & Nominal & Overwashed \\
\hline $\mathrm{Al}_{2} \mathrm{O}_{3}$ & 15.86 & 16.23 & 16.63 \\
\hline $\mathrm{BaO}$ & 0.26 & 0.27 & 0.28 \\
\hline $\mathrm{CaO}$ & 3.56 & 3.64 & 3.72 \\
\hline $\mathrm{CeO}_{2}$ & 0.44 & 0.45 & 0.45 \\
\hline $\mathrm{Cr}_{2} \mathrm{O}_{3}$ & 0.37 & 0.37 & 0.39 \\
\hline $\mathrm{CuO}$ & 0.20 & 0.22 & 0.22 \\
\hline $\mathrm{Fe}_{2} \mathrm{O}_{3}$ & 40.97 & 41.91 & 42.93 \\
\hline $\mathrm{K}_{2} \mathrm{O}$ & 0.40 & 0.42 & 0.42 \\
\hline $\mathrm{La}_{2} \mathrm{O}_{3}$ & 0.42 & 0.45 & 0.45 \\
\hline $\mathrm{MgO}$ & 0.22 & 0.23 & 0.23 \\
\hline $\mathrm{MnO}$ & 2.62 & 2.68 & 2.75 \\
\hline $\mathrm{Na}_{2} \mathrm{O}$ & 19.90 & 17.99 & 15.99 \\
\hline $\mathrm{NiO}$ & 1.74 & 1.78 & 1.83 \\
\hline $\mathrm{PbO}$ & 0.24 & 0.25 & 0.25 \\
\hline $\mathrm{SiO}_{2}$ & 1.92 & 1.96 & 2.01 \\
\hline $\mathrm{ThO}_{2}$ & 0.10 & 0.11 & 0.11 \\
\hline $\mathrm{U}_{3} \mathrm{O}_{8}$ & 9.71 & 9.94 & 10.20 \\
\hline $\mathrm{ZnO}$ & 0.38 & 0.40 & 0.41 \\
\hline $\mathrm{ZrO}_{2}$ & 0.73 & 0.75 & 0.77 \\
\hline
\end{tabular}


For the 50/50-blend MB3 option, the major components are $\mathrm{Fe}_{2} \mathrm{O}_{3}, \mathrm{Na}_{2} \mathrm{O}, \mathrm{Al}_{2} \mathrm{O}_{3}$, and $\mathrm{U}_{3} \mathrm{O}_{8}$. As the extent of washing increases, the $\mathrm{Na}_{2} \mathrm{O}$ concentration is reduced while the concentrations of the other oxides increases. Table 1 does not summarize the anions (or how they varied with sludge washing) associated with MB3. It is assumed that the anions will not have a negative impact or limit waste loadings in the model assessment activities. Experimental assessment of melt rate (Stone and Josephs 2001 and Lorier 2001) used a nonradioactive simulate MB3 sludge containing minor components (including the anions).

The potential for DWPF to receive an underwashed sludge is relatively high given current retrieval and/or tank farm space issues which may force a decision to minimize sludge washwater generation. This being the case, model assessments and frit development activities will be performed for both the nominal and underwashed sludge scenarios. As previously mentioned, one impact of sludge underwashing is a more $\mathrm{Na}_{2} \mathrm{O}$-rich sludge. The total alkali concentration will be dictated by the frit composition as well as the sludge washing scenario chosen. Glass formulation efforts will attempt to develop a frit that increases melt rate (relative to the use of Frit 200) for MB3 regardless of the sludge washing scenario but does not compromise product performance. It should be noted that the model assessments and frit development activities will not account for compositional variation in the sludge due to sampling and/or analytical errors, washing efficiency, blending issues and/or waste loading (WL) differences. These latter issues would be addressed in a separate study (e.g., variability study) assuming an alternative is recommended for MB3.

Another major component for $\mathrm{MB} 3$ is $\mathrm{U}_{3} \mathrm{O}_{8}$. As this program utilizes a developed test methodology to assess melt rate, the majority of the research will be performed using non-radioactive simulants (to minimize cost and schedule impacts). With $\mathrm{U}_{3} \mathrm{O}_{8}$ comprising approximately $10 \mathrm{wt} \%$ of the sludge, fabrication of a non-radioactive simulant must account for this major component being absent. Stone and Lambert (2001) discussed the fabrication of the non-radioactive MB3 simulants used to support this testing program. Frit development and model assessment activities will utilize the projected $\mathrm{U}_{3} \mathrm{O}_{8^{-}}$ containing MB3 composition (shown in Table 1). Laboratory tests to assess various glass property composition relationships will also be performed with uranium-bearing glasses to assess this issue and minimize risks.

\footnotetext{
2 It is noted that Frit 200 was not formulated for sludge-only processing. The term "baseline" refers to the current use of Frit 200 with MB2 and its planned use for MB3 (consistent with Harbour et al. 2000).
} 
The relatively high $\mathrm{Al}_{2} \mathrm{O}_{3}$ and $\mathrm{Fe}_{2} \mathrm{O}_{3}$ concentrations for $\mathrm{MB} 3$ may have an impact on both melt rate and $\mathrm{T}_{\mathrm{L}}$ predictions. In an effort to implement the "systems approach" to the fullest extent possible, this task is integrated with current efforts by SRTC to improve the $\mathrm{T}_{\mathrm{L}}$ correlation for DWPF (in an attempt to increase waste loadings). As the list of potential frits developed to increase melt rate is narrowed, the $T_{L}$ should be assessed with the new model to ensure the processing window is not limited. It should be noted that initial model assessments will be performed using the current $\mathrm{T}_{\mathrm{L}}$ model.

This task will utilize the compositional estimates provided by Elder (2000) for the blended MB3 option to assess compositional changes to the frit in an effort to increase melt rate. Based on programmatic direction, only the nominal and underwashed MB3 sludge scenarios will be evaluated.

\subsection{Frit DEVELOPMENT AND MODEL ASSESSMENTS ACTIVITIES}

The approach for the joint SRTC / PNNL frit development activity was to define a frit compositional envelope that when blended with MB3 may yield improved melt rates relative to the "baseline" Frit 200 / MB3 flowsheet. This joint effort utilized knowledge from previous research focused on frit optimization for DWPF (Jantzen 1988; Soper et al. 1983) and model predictions to assess various property composition relationships.

The overall strategy for the frit development activities was to explore frit compositional regions (both oxide components and ranges) that challenged "acceptable" predicted property behavior. Once major frit components were identified, ranges were established to push or challenge model predictions in an attempt to maximize melt rate. For example, an assessment on the extent that one could push total alkali content to increase melt rate but not have a negative effect on durability (either via model assessment and/or actual measurements) was addressed.

SRTC performed a series of calculations using models currently implemented in DWPF to assess the impacts of potential frit compositions on the projected operational window. The properties assessed included $\mathrm{T}_{\mathrm{L}}, \eta$, homogeneity, durability, and the constraints associated with the sum of alkali and/or $\mathrm{Al}_{2} \mathrm{O}_{3}$ concentrations (Edwards and Brown 1998; Peeler et al. 2000). These properties were assessed at the Property Acceptability Region (PAR) limits (Brown and Postles 1996).

PNNL conducted a similar series of calculations (utilizing existing Hanford property - composition models) to help define the frit compositional envelope that may yield improved melt rate for MB3. Both 
the SRTC and PNNL calculations were not limited to frit components that are currently used to fabricate DWPF frits, although a borosilicate glass system is required due to current Waste Acceptance Product Specifications (WAPS) criteria. In addition, the PNNL study did not take into account uncertainties associated with property and/or measurement errors. SRTC has the responsibility to ensure that a proposed alternative frit provides an adequate operational window and meets current DWPF acceptability criteria with the associated model error/uncertainties.

For consistency with the MB3 variability study (Harbour et al. 2000), initial SRTC and PNNL assessments used the nominal washed MB3 sludge composition with those oxides present in the sludge at concentrations that lead to amounts over $0.5 \mathrm{wt} \%$ in glass at sludge loadings up to $34 \mathrm{wt} \%$. As noted by Edwards (2000), trace components (elements whose oxides are present in the glass at concentrations less than $0.5 \mathrm{wt} \%$ ) are not expected to significantly impact durability and were omitted from the variability study. 3 It is noted that the current task focuses on melt rate for MB3 but the components expected to increase melt rate also have a significant impact on durability (in particular alkali). Table 2 shows the composition used in the SRTC / PNNL frit development and model assessment activities. It should also be noted that the composition used does not account for anions in glass (e.g., $\mathrm{SO}_{3}, \mathrm{Cl}, \mathrm{F}$ ) for either the nominal or underwashed sludge scenarios. It is assumed that the concentrations of these components in glass will not negatively affect melter processing, product performance, or limit waste loading (WL) by other single component constraints currently implemented in DWPF.

The lack of $\mathrm{Cr}_{2} \mathrm{O}_{3}$, which is known to increase the $\mathrm{T}_{\mathrm{L}}$ of many high-level waste (HLW) glasses, is probably the most questionable oxide. Based on Table 1, the maximum projected $\mathrm{Cr}_{2} \mathrm{O}_{3}$ content in the blended MB3 sludge (not considering the overwashed scenario) is $0.37 \mathrm{wt} \%$. At waste loadings of 25 to $35 \mathrm{wt} \%, \mathrm{Cr}_{2} \mathrm{O}_{3}$ levels in glass are projected to be 0.09 and $0.13 \mathrm{wt} \%$, respectively. These levels may be important in the final assessment of $\mathrm{T}_{\mathrm{L}}$ and the potential impacts on waste loading. Exclusion of these minor components from the paper studies will have minimal (if any) effect on model assessment or frit development activities regarding melt rate 4

3 Components not included: $\mathrm{Ba}, \mathrm{Ce}, \mathrm{Cr}, \mathrm{Cu}, \mathrm{K}, \mathrm{La}, \mathrm{Pb}$, Th, Zn, and Zr. See Plodinec, M.J., et al., "Technical Bases for the DWPF Glass Product Control Program (U),” WSRC-IM-91-116-5, Rev. 1, December 1995, pp. 68 - 71. It should also be noted that there are slight discrepancies between the values used by Harbour et al (2000) and those reported by Elder (2000) when the minor components are removed and the remaining components are renormalized.

${ }^{4} \mathrm{Cr}_{2} \mathrm{O}_{3}$ was added to the sludge simulant used in the laboratory tests and melt rate furnace tests. 


\section{Table 2. Composition (wt\%) of Nominal MB3 Sludge Used for Initial Model Assessments and Frit Development Activities.}

\begin{tabular}{||l|r||}
\hline \hline Oxide & Wt\% \\
\hline $\mathrm{Al}_{2} \mathrm{O}_{3}$ & 16.846 \\
\hline $\mathrm{CaO}$ & 3.743 \\
\hline $\mathrm{Cr}_{2} \mathrm{O}_{3}$ & 0.000 \\
\hline $\mathrm{Fe}_{2} \mathrm{O}_{3}$ & 43.087 \\
\hline $\mathrm{MgO}$ & 0.241 \\
\hline $\mathrm{MnO}$ & 2.824 \\
\hline $\mathrm{Na}_{2} \mathrm{O}$ & 18.740 \\
\hline $\mathrm{NiO}$ & 1.926 \\
\hline $\mathrm{SiO}_{2}$ & 2.018 \\
\hline $\mathrm{U}_{3} \mathrm{O}_{8}$ & 10.575 \\
\hline Total & 100.000 \\
\hline
\end{tabular}

The outcome of the SRTC/PNNL assessment and development activities 5 was a series of frit compositions (both components and percentages) that should maintain the projected waste loadings for the nominal MB3 sludge ( $25-28$ wt\% with Frit 200) based on model predictions while potentially increasing melt rate. The intent of this joint effort was to explore compositional extremes in frit space to bound the effect on melt rate for MB3. While exploring these compositional limits, model assessments were made but not necessarily used to limit the final compositional envelope. That is, given a specific frit composition, the frit was not excluded due to model predictions alone.

\footnotetext{
5 Although discussed separately, the assessments and frit development activities at SRTC and PNNL were highly integrated to
} minimize overlap and to ensure complementary outputs to meet the overall task objective. 


\subsection{FRIT DEVELOPMENT}

A series of frit compositions were generated which were expected to form acceptable glasses with 25.5 wt\% MB3 while challenging "acceptable" predicted property behavior. Glass and melt properties including glass $\eta$ at $1150^{\circ} \mathrm{C}\left(\eta_{1150}\right)$, electrical conductivity at $1150^{\circ} \mathrm{C}\left(\varepsilon_{1150}\right)$, normalized $\mathrm{Na}, \mathrm{B}$, and Li releases by the PCT $\left(\mathrm{r}_{\mathrm{Na}}, \mathrm{r}_{\mathrm{B}}\right.$, and $\mathrm{r}_{\mathrm{Li}}$, respectively), and $\mathrm{T}_{\mathrm{L}}$ (assuming a spinel primary crystalline phase) were predicted using coefficients from first-order expansion of glass properties in composition (listed in Table 3). The constraints placed on predicted properties (listed in Table 4) were generally more restrictive than those required for plant operation to account for model and waste composition uncertainties and in the case of $\eta_{1150}$, to allow for possible improvements in melt rate. A rough estimate of glasses propensity to form nepheline upon slow cooling, specifically, the normalized $\mathrm{SiO}_{2}$ concentration in the $\mathrm{SiO}_{2}-\mathrm{Na}_{2} \mathrm{O}$ $-\mathrm{Al}_{2} \mathrm{O}_{3}$ submixture ( $\mathrm{Li}$ et al. 1997), was calculated but was not found to restrict glass composition.

Figure 1 shows the initial frit compositions in a pair-wise plot or scatter plot matrix. These compositions adequately cover (in two dimensions) the area created by the bounds for each component in the frits with the exceptions of: (1) high $\mathrm{Al}_{2} \mathrm{O}_{3}$ and high $\mathrm{ZrO}_{2}$, (2) high $\mathrm{SiO}_{2}$ and low $\mathrm{Li}_{2} \mathrm{O}$, (3) high $\mathrm{SiO}_{2}$ and high $\mathrm{B}_{2} \mathrm{O}_{3}$, (4) high $\mathrm{SiO}_{2}$ and high $\mathrm{ZrO}_{2}$, (5) high $\mathrm{SiO}_{2}$ and high $\mathrm{Al}_{2} \mathrm{O}_{3}$, and (6) high $\mathrm{Na}_{2} \mathrm{O}$ and high $\mathrm{Li}_{2} \mathrm{O}$. Table 5 lists these initial frit compositions and the predicted properties that would result from melting each with $25.5 \mathrm{wt} \%$ of MB3. Each of the frits listed in Table 5 have acceptable properties (relative to those listed in Table 4) when blended with $25.5 \mathrm{wt} \%$ of the nominal washed MB3 (as shown in Table 2) based on the coefficients from the first-order expansion. This latter statement assumes that the minor components that were not used in this initial assessment have a minimal (or no) impact on these properties. As previously mentioned, of particular interest is the impact of $\mathrm{Cr}_{2} \mathrm{O}_{3}$ on $\mathrm{T}_{\mathrm{L}}$ predictions since it is absent in the sludge composition used for model assessments. 
Table 3. First-Order Coefficients for Selected Glass Properties.

\begin{tabular}{|c|c|c|c|c|c|c|c|c|c|c|c|c|c|c|c|}
\hline \multirow[b]{2}{*}{ Oxide $^{(a)}$} & \multicolumn{6}{|c|}{$\begin{array}{c}\text { PCT Durability } \\
\left(\mathrm{g} / \mathrm{m}^{2}\right)\end{array}$} & \multirow{2}{*}{$\begin{array}{c}\begin{array}{c}\text { Liquidus } \\
\left({ }^{\circ} \mathbf{C}\right)\end{array} \\
\mathbf{T}_{\mathbf{L}}{ }^{(\mathbf{c}, \mathbf{d})} \\
\text { new } \\
\end{array}$} & \multicolumn{5}{|c|}{$\begin{array}{c}\text { Viscosity } \\
\text { (Pa-s) }\end{array}$} & \multicolumn{3}{|c|}{$\begin{array}{c}\text { Electrical Conductivity } \\
(\mathrm{S} / \mathrm{m})\end{array}$} \\
\hline & $\begin{array}{c}\ln \left(\mathbf{r}_{\mathrm{B}}\right),^{(\mathbf{b})} \\
\text { new }\end{array}$ & $\begin{array}{c}\ln \left(\mathbf{r}_{\mathrm{Li}}\right) \\
\text { new } \\
\end{array}$ & $\begin{array}{c}\ln \left(\mathbf{r}_{\mathrm{Na}}\right) \\
\text { new } \\
\end{array}$ & $\begin{array}{c}\operatorname{Ln}\left(\mathbf{r}_{\mathbf{B}}\right) \\
\mathrm{CVS} \\
\end{array}$ & $\begin{array}{c}\ln \left(\mathbf{r}_{\mathrm{Li}}\right) \\
\text { CVS } \\
\end{array}$ & $\begin{array}{c}\ln \left(\mathbf{r}_{\mathrm{Na}}\right) \\
\mathbf{C V S} \\
\end{array}$ & & $\begin{array}{c}A_{\eta}(e, f) \\
\text { new }\end{array}$ & $\mathbf{B}_{\text {n }}$, new & \begin{tabular}{c|}
$\mathbf{A}_{\eta}$, \\
$\mathbf{C V S}$ \\
\end{tabular} & $\mathrm{B}_{\eta}, \mathrm{CVS}$ & $\begin{array}{l}\ln \left(\eta_{1150}\right) \\
\text { CVS }\end{array}$ & \begin{tabular}{l|}
$\mathbf{A}_{\boldsymbol{\varepsilon}}(\mathrm{g})$ \\
$\mathrm{CVS}$ \\
\end{tabular} & $\mathbf{B}_{\varepsilon}, \mathbf{C V S}$ & $\begin{array}{c}\ln \left(\varepsilon_{1150}\right) \\
\text { CVS } \\
\end{array}$ \\
\hline $\mathrm{Al}_{2} \mathrm{O}_{3}$ & -32.132 & -29.331 & -32.858 & -41.077 & -36.078 & -41.070 & 3222.386 & -2.860 & 27599.0 & -1.242 & 25113.0 & 17.088 & 6.260 & -8646.92 & 0.170 \\
\hline $\mathrm{B}_{2} \mathrm{O}_{3}$ & 14.509 & 11.958 & 9.957 & 13.009 & 11.044 & 10.237 & 197.575 & -13.594 & 8765.00 & -13.096 & 9054.08 & -6.842 & 12.960 & -15483.0 & 2.153 \\
\hline $\mathrm{CaO}$ & -12.976 & -9.121 & -4.018 & -7.473 & -4.629 & -1.770 & 1183.426 & -25.804 & 27511.0 & -20.768 & 20359.0 & -6.279 & 13.820 & -17621.0 & 1.600 \\
\hline $\mathrm{Cr}_{2} \mathrm{O}_{3}$ & & & & & & & 31677.695 & & & & & & & & \\
\hline $\mathrm{Fe}_{2} \mathrm{O}_{3}$ & -6.740 & -9.463 & -9.110 & -9.027 & -12.051 & -10.975 & 3651.072 & -3.490 & -835.00 & 0.934 & -5306.61 & -2.525 & 10.870 & -13527.0 & 1.778 \\
\hline $\mathrm{K}_{2} \mathrm{O}$ & -10.312 & -6.515 & 0.323 & & & & -929.671 & -16.589 & 14436.0 & & & & & & \\
\hline $\mathrm{Li}_{2} \mathrm{O}$ & 9.558 & 9.507 & 7.328 & 10.431 & 8.487 & 8.688 & 307.915 & -7.100 & -10377.0 & -6.255 & -12639.0 & -15.030 & 7.770 & 6863.36 & 12.694 \\
\hline $\mathrm{MgO}$ & 0.399 & -0.484 & 2.575 & 7.044 & 4.592 & 7.483 & 2664.278 & -19.102 & 25120.0 & -17.677 & 23268.0 & -1.198 & 9.970 & -11589.0 & 2.974 \\
\hline $\mathrm{MnO}$ & -18.462 & -14.389 & -16.326 & & & & -124.103 & & & & & & & & \\
\hline $\mathrm{Na}_{2} \mathrm{O}$ & 16.821 & 13.193 & 18.873 & 17.258 & 13.719 & 18.937 & -440.469 & -9.974 & 632.00 & -8.780 & -2002.31 & -10.686 & 5.820 & 7157.78 & 10.844 \\
\hline $\mathrm{NiO}$ & & & & & & & 13399.326 & & & & & & & & \\
\hline $\mathrm{SiO}_{2}$ & -4.410 & -3.605 & -4.278 & -3.917 & -2.904 & -3.994 & 1151.831 & -10.136 & 26427.2 & -11.711 & 28819.0 & 8.498 & 8.410 & -10470.0 & 0.996 \\
\hline $\mathrm{U}_{3} \mathrm{O}_{8}$ & & & & & & & 3060.342 & & & & & & & & \\
\hline $\mathrm{ZrO}_{2}$ & -14.976 & -13.177 & -18.384 & -21.246 & -20.152 & -22.752 & 3975.649 & -55.621 & 95153.0 & -51.386 & 90415.0 & 12.811 & 7.310 & -11830.0 & -0.867 \\
\hline Others & & & & -1.067 & 0.247 & -2.825 & & & & -26.515 & 29680.0 & -3.561 & 32.060 & -39574.0 & 3.942 \\
\hline $\mathrm{LN}_{2} \mathrm{O}_{3}{ }^{\mathrm{h}}$ & & & & & & & & 43.460 & -78677.0 & & & & & & \\
\hline
\end{tabular}

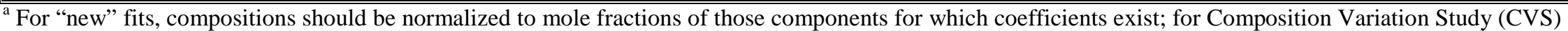

fits, compositions should be in mole fractions of components with all components without coefficients contained in "Others".

${ }^{\mathrm{b}}$ The units assumed for $\mathrm{r}_{\mathrm{i}}$ are $\mathrm{g} \cdot \mathrm{m}^{-2}$.

${ }^{c}$ For the $\mathrm{T}_{\mathrm{L}}$ fit, normalized mole fractions of cations are used rather than oxides.

${ }^{\mathrm{d}}$ The units assumed for $\mathrm{T}_{\mathrm{L}}$ are ${ }^{\circ} \mathrm{C}$.

${ }^{\mathrm{e}} \mathrm{A}_{\alpha}$ and $\mathrm{B}_{\alpha}$ are Arrhenius parameters such that $\operatorname{Ln}(\alpha)=\mathrm{A}+\mathrm{B} / \mathrm{T}$, where, $\alpha$ is the property and $\mathrm{T}$ is absolute temperature.

${ }^{\mathrm{f}}$ The units assumed for $\eta$ are $\mathrm{Pa} \cdot \mathrm{s}$.

${ }^{\mathrm{g}}$ The units assumed for $\varepsilon$ are $\mathrm{S} \cdot \mathrm{m}^{-1}$.

${ }^{\mathrm{h}} \mathrm{LN}_{2} \mathrm{O}_{3}$ represents total three valent lanthanide oxides and yttria $\left(\mathrm{Y}_{2} \mathrm{O}_{3}+\mathrm{La}_{2} \mathrm{O}_{3}+\mathrm{Ce}_{2} \mathrm{O}_{3}+\operatorname{Pr}_{2} \mathrm{O}_{3}+\mathrm{Nd}_{2} \mathrm{O}_{3}+\mathrm{Sm}_{2} \mathrm{O}_{3}+\mathrm{Gd}_{2} \mathrm{O}_{3}\right)$. 
Table 4. Constraints Used in This Study on Predicted Glass Properties.

\begin{tabular}{|c|c|c|c||}
\hline Property & Lower Limit & Upper Limit & Units \\
\hline \hline $\mathrm{r}_{\mathrm{B}}$ & - & $4(2)$ & $\mathrm{g} / \mathrm{L}\left(\mathrm{g} / \mathrm{m}^{2}\right)$ \\
\hline $\mathrm{r}_{\mathrm{Li}}$ & - & $4(2)$ & $\mathrm{g} / \mathrm{L}\left(\mathrm{g} / \mathrm{m}^{2}\right)$ \\
\hline $\mathrm{r}_{\mathrm{Na}}$ & - & $4(2)$ & $\mathrm{g} / \mathrm{L}\left(\mathrm{g} / \mathrm{m}^{2}\right)$ \\
\hline$\eta_{1150}$ & $30(3.0)$ & $45(4.5)$ & Poise $(\mathrm{Pa}-\mathrm{s})$ \\
\hline $\mathrm{T}_{\mathrm{L}}$ & - & 1000 & ${ }^{\circ} \mathrm{C}$ \\
\hline \hline
\end{tabular}

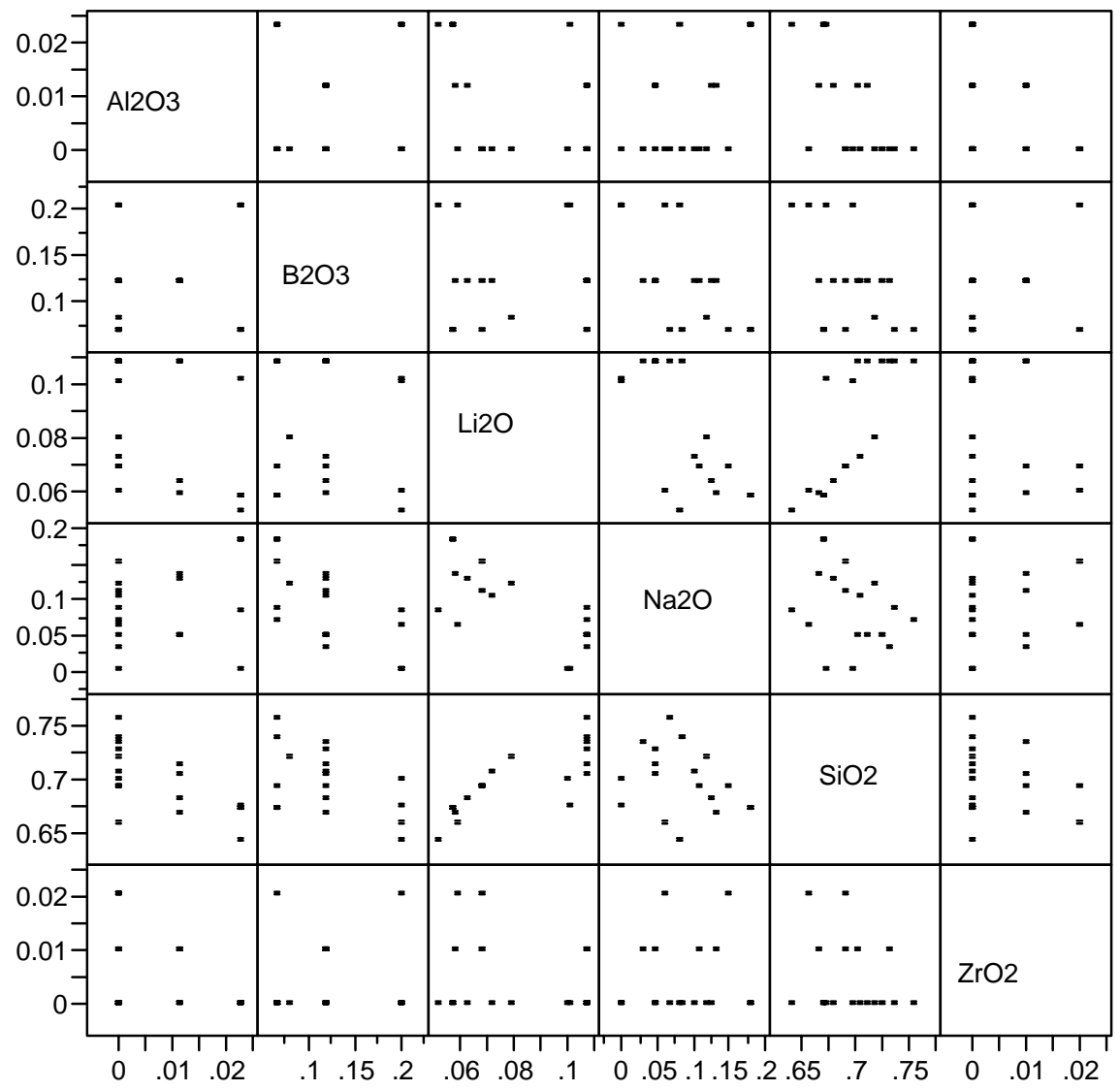

Figure 1. Pair-wise Plots (Scatter Plot Matrix) of Calculated Frit Compositions (in weight fractions). 
Table 5. Frit Compositions (in wt\%) and Resultant Glass Property Predictions.

\begin{tabular}{|c|c|c|c|c|c|c|c|c|c|c|c|c|c|c|c|c|c|c|c|}
\hline Frit ID & $\begin{array}{l}\text { Frit } \\
302 \\
\end{array}$ & $\begin{array}{l}\text { Frit } \\
303 \\
\end{array}$ & $\begin{array}{l}\text { Frit } \\
304 \\
\end{array}$ & $\begin{array}{l}\text { Frit } \\
305\end{array}$ & $\begin{array}{l}\text { Frit } \\
306 \\
\end{array}$ & $\begin{array}{l}\text { Frit } \\
307\end{array}$ & $\begin{array}{l}\text { Frit } \\
308 \\
\end{array}$ & $\begin{array}{l}\text { Frit } \\
309 \\
\end{array}$ & $\begin{array}{l}\text { Frit } \\
310 \\
\end{array}$ & $\begin{array}{c}\text { Frit } \\
3117\end{array}$ & $\begin{array}{l}\text { Frit } \\
312 \\
\end{array}$ & $\begin{array}{l}\text { Frit } \\
313 \\
\end{array}$ & $\begin{array}{l}\text { Frit } \\
314\end{array}$ & $\begin{array}{l}\text { Frit } \\
315 \\
\end{array}$ & $\begin{array}{l}\text { Frit } \\
316\end{array}$ & $\begin{array}{l}\text { Frit } \\
317 \\
\end{array}$ & $\begin{array}{l}\text { Frit } \\
318 \\
\end{array}$ & $\begin{array}{l}\text { Frit } \\
319 \\
\end{array}$ & $\begin{array}{l}\text { Frit } \\
320 \\
\end{array}$ \\
\hline $\mathrm{Al}_{2} \mathrm{O}_{3}$ & 2.29 & 2.29 & 2.29 & 0.00 & 0.00 & 0.00 & 2.29 & 0.00 & 1.14 & 1.14 & 0.00 & 0.00 & 0.00 & 0.00 & 0.00 & 0.00 & 1.14 & 1.14 & 0.00 \\
\hline $\mathrm{B}_{2} \mathrm{O}_{3}$ & 20.13 & 20.13 & 6.71 & 6.71 & 12.08 & 12.08 & 6.71 & 8.07 & 12.00 & 12.00 & 6.71 & 6.71 & 20.13 & 20.13 & 12.00 & 12.00 & 12.00 & 12.00 & 8.00 \\
\hline $\mathrm{Li}_{2} \mathrm{O}$ & 5.19 & 10.12 & 5.80 & 10.74 & 7.22 & 10.74 & 5.80 & 7.91 & 6.27 & 10.74 & 6.89 & 10.74 & 5.94 & 10.03 & 10.74 & 6.82 & 5.87 & 10.74 & 8.00 \\
\hline $\mathrm{Na}_{2} \mathrm{O}$ & 8.28 & 0.00 & 18.07 & 6.93 & 10.24 & 4.67 & 18.07 & 12.00 & 12.51 & 4.83 & 15.18 & 8.75 & 6.12 & 0.00 & 3.07 & 11.06 & 13.28 & 4.72 & 12.00 \\
\hline $\mathrm{SiO}_{2}$ & 64.11 & 67.46 & 67.13 & 75.62 & 70.46 & 72.51 & 67.13 & 72.01 & 68.07 & 71.28 & 69.21 & 73.80 & 65.79 & 69.84 & 73.19 & 69.12 & 66.71 & 70.39 & 72.00 \\
\hline $\mathrm{ZrO}_{2}$ & 0.00 & 0.00 & 0.00 & 0.00 & 0.00 & 0.00 & 0.00 & 0.00 & 0.00 & 0.00 & 2.01 & 0.00 & 2.01 & 0.00 & 1.00 & 1.00 & 1.00 & 1.00 & 0.00 \\
\hline $\mathrm{MgO}$ & 0.00 & 0.00 & 0.00 & 0.00 & 0.00 & 0.00 & 0.00 & 0.00 & 0.00 & 0.00 & 0.00 & 0.00 & 0.00 & 0.00 & 0.00 & 0.00 & 0.00 & 0.00 & 0.00 \\
\hline $\mathrm{r}_{\mathrm{B}}\left(\mathrm{g} / \mathrm{m}^{2}\right)$, new & 2.0 & 1.3 & 1.8 & 0.9 & 1.7 & 1.3 & 1.8 & 1.5 & 1.9 & 1.2 & 1.7 & 1.3 & 2.0 & 1.6 & 1.0 & 1.8 & 1.9 & 1.1 & 1.5 \\
\hline $\mathrm{r}_{\mathrm{Li}}\left(\mathrm{g} / \mathrm{m}^{2}\right)$, new & 1.5 & 1.2 & 1.3 & 1.0 & 1.4 & 1.3 & 1.3 & 1.3 & 1.4 & 1.1 & 1.3 & 1.2 & 1.5 & 1.5 & 1.0 & 1.4 & 1.4 & 1.1 & 1.3 \\
\hline$r_{\mathrm{Na}}\left(\mathrm{g} / \mathrm{m}^{2}\right)$, new & 1.1 & 0.5 & 1.9 & 0.7 & 1.3 & 0.8 & 1.9 & 1.3 & 1.4 & 0.7 & 1.6 & 1.0 & 1.1 & 0.7 & 0.6 & 1.3 & 1.5 & 0.7 & 1.3 \\
\hline$r_{B}\left(g / m^{2}\right), C V S$ & 1.8 & 1.2 & 2.0 & 1.2 & 2.0 & 1.6 & 2.0 & 1.9 & 2.0 & 1.3 & 2.0 & 1.6 & 1.9 & 1.7 & 1.2 & 2.0 & 2.0 & 1.3 & 1.9 \\
\hline $\mathrm{r}_{\mathrm{Li}}\left(\mathrm{g} / \mathrm{m}^{2}\right), \mathrm{CVS}$ & 1.4 & 1.1 & 1.5 & 1.1 & 1.6 & 1.4 & 1.5 & 1.5 & 1.6 & 1.2 & 1.5 & 1.3 & 1.5 & 1.5 & 1.0 & 1.6 & 1.5 & 1.1 & 1.5 \\
\hline $\mathrm{r}_{\mathrm{Na}}\left(\mathrm{g} / \mathrm{m}^{2}\right), \mathrm{CVS}$ & 1.2 & 0.6 & 1.9 & 0.9 & 1.5 & 1.0 & 1.9 & 1.5 & 1.6 & 0.8 & 1.7 & 1.2 & 1.2 & 0.9 & 0.7 & 1.5 & 1.6 & 0.8 & 1.5 \\
\hline $\begin{array}{l}\eta_{\mathrm{T}} @ 1150^{\circ} \mathrm{C} \\
(\mathrm{Pa} \cdot \mathrm{s}), \text { new }\end{array}$ & 4.5 & 3.1 & 4.5 & 4.5 & 4.5 & 3.3 & 4.5 & 4.5 & 4.5 & 3.3 & 4.5 & 3.5 & 4.5 & 3.1 & 4.1 & 4.5 & 4.5 & 3.3 & 4.4 \\
\hline $\begin{array}{l}\eta_{1150}(\mathrm{~Pa} \cdot \mathrm{s}) \\
\mathrm{CVS}\end{array}$ & 4.3 & 3.1 & 3.7 & 3.9 & 4.0 & 3.0 & 3.7 & 3.9 & 4.0 & 3.0 & 3.8 & 3.0 & 4.4 & 3.0 & 3.8 & 4.0 & 4.0 & 3.0 & 3.8 \\
\hline $\mathrm{T}_{\mathrm{L}}\left({ }^{\circ} \mathrm{C}\right)$, new & 912 & 971 & 856 & 930 & 884 & 922 & 856 & 879 & 879 & 935 & 868 & 901 & 918 & 941 & 955 & 885 & 879 & 944 & 878 \\
\hline
\end{tabular}

\footnotetext{
6 Predicted properties are based on a nominally washed MB3 sludge at $25.5 \mathrm{wt} \%$ waste loading.

${ }^{7}$ It should be noted that Frit 311 as defined in Table 5, does not correspond to the Frit 311 developed by Jantzen (1998).
} 
To validate the predictions (using the coefficients from the first-order expansion from Table 3), a glass (Frit 320 with 25.5 wt\% MB3 nominally washed sludge) was fabricated at the Savannah River Technology Center (SRTC) and various properties measured (durability and $\eta$ ). . SRTC measured the glass composition, $\mathrm{r}_{\mathrm{B}}, \mathrm{r}_{\mathrm{Li}}, \mathrm{r}_{\mathrm{Na}}$, and $\eta_{1150}$. Table 6 shows the target and measured compositions (wt $\%$ ) of MB3N320q.

Table 6. Target and Measured Composition (wt\%) of MB3N320q at $25.5 \mathrm{wt} \%$ Loading.

\begin{tabular}{|l|c|c||}
\hline Oxide & Target & Measured \\
\hline \hline $\mathrm{Al}_{2} \mathrm{O}_{3}$ & 4.296 & 4.27 \\
\hline $\mathrm{B}_{2} \mathrm{O}_{3}$ & 5.96 & 5.73 \\
\hline $\mathrm{CaO}$ & 0.954 & 0.21 \\
\hline $\mathrm{Fe}_{2} \mathrm{O}_{3}$ & 10.987 & 9.91 \\
\hline $\mathrm{Li}_{2} \mathrm{O}$ & 5.96 & 5.33 \\
\hline $\mathrm{MgO}$ & 0.061 & 0.0 \\
\hline $\mathrm{MnO}$ & 0.72 & 0.73 \\
\hline $\mathrm{Na}_{2} \mathrm{O}$ & 13.719 & 11.7 \\
\hline $\mathrm{NiO}^{2}$ & 0.491 & 0.45 \\
\hline $\mathrm{SiO}$ & 54.155 & 54.3 \\
\hline $\mathrm{U}_{3} \mathrm{O}_{8}$ & 2.697 & 2.51 \\
\hline Total & 100.00 & 95.14 \\
\hline \hline
\end{tabular}

8 Glasses produced in this study will have the following nomenclature:

$$
\begin{aligned}
& \text { MB3 - to identify MB3 sludge } \\
& \text { N or } \mathrm{U}-\text { to identify nominal or underwashed sludge } \\
& 301-326 \text { - to identify the frit composition } \\
& \text { q or clc - to identify the specific heat treatment (quenched or centerline cooled) }
\end{aligned}
$$


Viscosity (as a function of temperature) and durability (as defined by the PCT) were measured on this particular uranium-bearing glass. Table 7 compares the measured and predicted (based on target composition using the coefficients from the first-order expansion) property values. Normalized elemental releases for $\mathrm{B}, \mathrm{Na}$, and $\mathrm{Li}$ are shown based on measured compositions. The calculations were found to over predict PCT releases (making the calculations conservative) and slightly underpredict $\eta_{1150}$. The $T_{L}$ was not measured.

Table 7. Comparison of Predicted and Measured Property Values for MB3N320q at 25.5 wt\% Loading.

\begin{tabular}{||l|c|c|c|c||}
\hline \hline Property & $\begin{array}{c}\text { Predicted } \\
\text { Value }\end{array}$ & $\begin{array}{c}\text { Measured } \\
\text { Values }\end{array}$ & $\begin{array}{c}\text { Imposed } \\
\text { Limit }\end{array}$ & Unit \\
\hline $\mathrm{r}_{\mathrm{B}}$ & $3.0-3.8$ & 1.12 & $<4$ & $\mathrm{~g} / \mathrm{L}$ \\
\hline $\mathrm{r}_{\mathrm{Li}}$ & $2.6-3.0$ & 1.44 & $<4$ & $\mathrm{~g} / \mathrm{L}$ \\
\hline $\mathrm{r}_{\mathrm{Na}}$ & $2.6-3.0$ & 1.52 & $<4$ & $\mathrm{~g} / \mathrm{L}$ \\
\hline$\eta_{1150 \text { (Poise) }}$ & 38 & 44.8 & $30-45$ & Poise \\
\hline$\eta_{1150 \text { (Pa-s) }}$ & $3.8-4.4$ & 4.48 & $3-4.5$ & $\mathrm{~Pa} \cdot \mathrm{s}$ \\
\hline $\mathrm{T}_{\mathrm{L}}$ & 878 & not measured & $<1000$ & ${ }^{\circ} \mathrm{C}$ \\
\hline
\end{tabular}

A second series of candidate frit compositions were developed using compositional guidelines to supplement those listed in Table 5.PThese guidelines included: an upper limit on total alkali in glass of approximately $20 \mathrm{wt} \%$, an upper limit of $\mathrm{B}_{2} \mathrm{O}_{3}$ in glass of $11 \mathrm{wt} \%$, and no $\mathrm{Al}_{2} \mathrm{O}_{3}$ in frit due to the presence of $\mathrm{Al}_{2} \mathrm{O}_{3}$ in sludge at relatively high concentrations. The resulting compositions, which explored compositional alternatives to the existing set are discussed below. Table 8 provides the compositions (in $\mathrm{wt} \%$ ) for the additional frits.

(i) Frit 322 - a "refractory frit" composition. As discussed by Peeler et al. (2001), one option to minimize foam production is to delay the onset of the initial liquid phase formation until off-gas generation is "complete". This may be accomplished by the use of a "refractory"

${ }^{9}$ Although guidelines were established, they did not restrict previous frits (as shown in Table 5) from being considered as candidate frits to improve melt rate. These guidelines were only applied to the development of the supplemental frit compositions. 
frit. Frit 322 contains $77 \mathrm{wt} \% \mathrm{SiO}_{2}$ and its corresponding glass is predicted to have a relatively high $\eta\left(75\right.$ Poise at $\left.1150^{\circ} \mathrm{C}\right)$.

(ii) Frit 323 - which included adjustments to Frit 302 (shown in Table 5) that addressed concerns of the relatively high $\mathrm{B}_{2} \mathrm{O}_{3}$ content as well as the presence of $\mathrm{Al}_{2} \mathrm{O}_{3}$. One of the concerns was the potential to produce an amorphous phase separated glass if the $\mathrm{B}_{2} \mathrm{O}_{3}$ content exceeded $11-12 \mathrm{wt} \%$ in glass. Another concern was the presence of $\mathrm{Al}_{2} \mathrm{O}_{3}$ in Frit 302 given that $15-17 \mathrm{wt} \% \mathrm{Al}_{2} \mathrm{O}_{3}$ is present in the $\mathrm{MB} 3$ sludge. Speculation was that the presence of additional $\mathrm{Al}_{2} \mathrm{O}_{3}$ in the frit would impede melt rate. The compositional adjustments for Frit 323 were simply to lower the $\mathrm{B}_{2} \mathrm{O}_{3}$ content to $15 \%$ in frit (projected to be $\sim 11.5 \mathrm{wt} \%$ in glass at $25 \%$ waste loading), remove the $\mathrm{Al}_{2} \mathrm{O}_{3}$, and replace the difference (on a mass basis) by adding more $\mathrm{SiO}_{2}$.

(iii) Frit 324 - which included adjustments to evaluate if the $\mathrm{Li}_{2} \mathrm{O}$ or $\mathrm{Na}_{2} \mathrm{O}$ content for a specific composition influenced melt rate. The difference between Frit 323 and Frit 324 is an exchange of the $\mathrm{Li}_{2} \mathrm{O}$ and $\mathrm{Na}_{2} \mathrm{O}$ contents.

(iv) Frit 325 - a composition developed to address a specific DWPF request regarding the potential to couple Frit 202 (currently in inventory) with another frit to improve melt rate while working off the current inventory.

(v) Frit 326 - a composition to address a concern that there were only a limited number of proposed frit compositions that include $\mathrm{MgO}$. It was suggested that $\mathrm{MgO}$ should be included in the frit at the same level as in Frit 165 (i.e., $1 \mathrm{wt} \%$ in frit) as it may have a positive impact on durability. To address this issue as well as to evaluate whether the addition of $1 \% \mathrm{MgO}$ to any of the frits would have an impact on the assessment of melt rate, $1 \mathrm{wt} \% \mathrm{Na}_{2} \mathrm{O}$ was replaced by $1 \% \mathrm{MgO}$ in Frit 320 leading to Frit 326 . This frit should provide for an assessment of whether the addition of $1 \% \mathrm{MgO}$ or a $1 \%$ reduction of $\mathrm{Na}_{2} \mathrm{O}$ impedes melt rate or lowers durability relative to Frit 320 (see Stone and Josephs 2001). 
Table 8. Frit Compositions (wt\%) of Existing Frits and Those Developed To Supplement the Existing Database.

\begin{tabular}{||c|c|c|c|c|c|c|c|c||}
\hline Oxide & Frit 200 & Frit 165 & $\begin{array}{c}\text { Frit 165 w/o } \\
\mathbf{Z r O}_{2}\end{array}$ & Frit 322 & Frit 323 & Frit 324 & Frit 325 & Frit 326 \\
\hline \hline $\mathrm{Al}_{2} \mathrm{O}_{3}$ & 0.00 & 0.00 & 0.00 & 0.00 & 0.00 & 0.00 & 0.00 & 0.00 \\
\hline $\mathrm{B}_{2} \mathrm{O}_{3}$ & 12.00 & 10.00 & 10.10 & 8.00 & 15.00 & 15.00 & 9.10 & 8.00 \\
\hline $\mathrm{Li}_{2} \mathrm{O}$ & 5.00 & 7.00 & 7.07 & 5.00 & 5.19 & 8.28 & 8.10 & 8.00 \\
\hline $\mathrm{Na}_{2} \mathrm{O}$ & 11.00 & 13.00 & 13.13 & 10.00 & 8.28 & 5.19 & 12.20 & 11.00 \\
\hline $\mathrm{SiO}_{2}$ & 70.00 & 68.00 & 68.69 & 77.00 & 71.53 & 71.53 & 68.60 & 72.00 \\
\hline $\mathrm{ZrO}$ & 0.00 & 1.00 & 0.00 & 0.00 & 0.00 & 0.00 & 0.00 & 0.00 \\
\hline $\mathrm{MgO}$ & 2.00 & 1.00 & 1.01 & 0.00 & 0.00 & 0.00 & 1.00 & 1.00 \\
\hline \hline
\end{tabular}

Table 8 includes Frit 200 (considered to be the baseline frit) and Frit 165 (a sludge-only frit developed by Soper et al. [1983]). It should be noted that Frit 165 with and without $\mathrm{ZrO}_{2}$ is also shown in Table 8 and will be evaluated by PCCS model assessments as well as via the suite of melt rate tests. The Frit 165 without $\mathrm{ZrO}_{2}$ option surfaced as it may prove beneficial to DWPF. It should be noted that the PCCS model assessments for Frit 200 and Frit 165 (as discussed in Section 5.2) are based on target compositions as defined by Fowler et al. (1991) and do not include allowable compositional tolerances of minor components.

\subsection{PCCS MOdel ASSESSMENT OF CANDIDATE Frits}

Lorier (2001) discussed the initial down selection process 10 used to narrow the 27 potential frit compositions (shown in Table 5 and 8) down to a more manageable size (i.e., 15). Again, it should also be noted that the PCCS assessments were based on the MB3 sludge composition shown in Table 2 (which includes uranium, but excludes the minor components for consistency with the variability study [Harbour et al. 2000]). Exclusion of these components from the paper studies should have minimal (if any) effect on model assessment or frit development activities regarding melt rate. Although the objective of this task is to improve melt rate for MB3, one must continually assess other properties which are critical to DWPF operations.

10 The initial down selection process was primarily based on the compositional guidelines established by SRTC, laboratory scale crucible scale tests and initial melt rate furnace testing. Refer to Section 5.1 for a discussion of the compositional guidelines. 
Table 9 summarizes the 15 candidate frits compositions and the estimated properties (using existing PCCS model predictions) for both the nominal and underwashed MB3 sludge scenarios. The estimated property values provided are those at the maximum allowable waste loading as defined by the current PCCS model predictions at the PAR. Table 10 provides the predicted properties at a fixed waste loading (25.5 wt $\%$ so that various properties (e.g., $\eta$ or durability) can be directly compared. Definition of acceptable properties for this assessment are based on PAR limit values (see Table 11, Brown and Postles [1996]) for the respective properties.

11 For the non-radioactive laboratory and melt rate furnace test, the required amount of slurry receipt and adjustment tank (SRAT) product was combined with the required amount of frit based on a waste loading of 23.2 $\mathrm{wt} \%$. This is equivalent to a waste laoding of $\sim 25.5 \mathrm{wt} \%$ in glass for the uranium-containing glasses. 
Table 9. Candidate Frit Compositions (wt\%) and Estimated Properties 12 at the

Maximum Allowable Waste Loading For Nominal and Underwashed MB3 Sludge.

\begin{tabular}{|c|c|c|c|c|c|c|c|c|c|c|c|c|c|c|c|}
\hline Oxide & Frit 165 & $\begin{array}{c}\text { Frit } 165 \\
\text { w/o } \mathrm{ZrO}_{2} \\
\end{array}$ & Frit 200 & Frit 303 & Frit 304 & Frit 307 & Frit 313 & Frit 314 & Frit 315 & Frit 320 & Frit 322 & Frit 323 & Frit 324 & Frit 325 & Frit 326 \\
\hline $\mathrm{Al}_{2} \mathrm{O}_{3}$ & 0.00 & 0.00 & 0.00 & 2.29 & 2.29 & 0.00 & 0.00 & 0.00 & 0.00 & 0.00 & 0.00 & 0.00 & 0.00 & 0.00 & 0.00 \\
\hline $\mathrm{B}_{2} \mathrm{O}_{3}$ & 10.00 & 10.10 & 12.00 & 20.13 & 6.71 & 12.08 & 6.71 & 20.13 & 20.13 & 8.00 & 8.00 & 15.00 & 15.00 & 8.55 & 8.00 \\
\hline $\mathrm{Li}_{2} \mathrm{O}$ & 7.00 & 7.07 & 5.00 & 10.12 & 5.80 & 10.74 & 10.74 & 5.94 & 10.03 & 8.00 & 5.00 & 5.19 & 8.28 & 7.55 & 8.00 \\
\hline $\mathrm{Na}_{2} \mathrm{O}$ & 13.00 & 13.13 & 11.00 & 0.00 & 18.07 & 4.67 & 8.75 & 6.12 & 0.00 & 12.00 & 10.00 & 8.28 & 5.19 & 9.10 & 11.00 \\
\hline $\mathrm{SiO}_{2}$ & 68.00 & 68.69 & 70.00 & 67.46 & 67.13 & 72.51 & 73.80 & 65.79 & 69.84 & 72.00 & 77.00 & 71.53 & 71.53 & 72.80 & 72.00 \\
\hline $\mathrm{ZrO}_{2}$ & 1.00 & 0.00 & 0.00 & 0.00 & 0.00 & 0.00 & 0.00 & 2.01 & 0.00 & 0.00 & 0.00 & 0.00 & 0.00 & 0.00 & 0.00 \\
\hline $\mathrm{MgO}$ & 1.00 & 1.01 & 2.00 & 0.00 & 0.00 & 0.00 & 0.00 & 0.00 & 0.00 & 0.00 & 0.00 & 0.00 & 0.00 & 1.50 & 1.00 \\
\hline
\end{tabular}

\section{Nominal Wash Case}

\begin{tabular}{|c|c|c|c|c|c|c|c|c|c|c|c|c|c|c|c|}
\hline WL Range & $23.0-29.0$ & $22.5-29.0$ & $23.0-29.5$ & $21.0-28.0$ & 0 & $22.0-30.5$ & $22.0-30.5$ & $23.0-28.5$ & $22.0-29.5$ & $22.0-30.0$ & $26.5-31.5$ & $22.0-30.0$ & $22.0-30.0$ & $23.0-30.5$ & $22.5-30.0$ \\
\hline MAX WL & 29 & 29 & 29.5 & 28 & Restricted & 30.5 & 30.5 & 28.5 & 29.5 & 30 & 31.5 & 30 & 30 & 30.5 & 30 \\
\hline Viscosity (Poise) & 28.3 & 28.6 & 51.8 & 40.4 & & 33.1 & 30.4 & 40.4 & 38.2 & 33.5 & 83.7 & 59.9 & 43.8 & 47.7 & 36.8 \\
\hline $\mathrm{T}_{\mathrm{L}}\left({ }^{\circ} \mathrm{C}\right)$ & 1022.3 & 1019.8 & 1021.1 & 1023.9 & & 1024.2 & 1019.8 & 1024.4 & 1021.6 & 1020 & 1020.8 & 1021.7 & 1021.7 & 1023.2 & 1020 \\
\hline$\Delta \mathrm{G}_{\mathrm{P}}$ & -12.04 & -12.24 & -10.01 & -6.39 & & -9.08 & -10.89 & -8.22 & -6.85 & -11.54 & -8.4 & -8.48 & -8.37 & -9.68 & -11.05 \\
\hline Homo & 225.5 & 226.6 & 226.7 & 232.1 & & 231.3 & 231.3 & 224.3 & 229 & 230.1 & 233.6 & 230.1 & 230.1 & 229.1 & 229 \\
\hline Alkali (wt\%) & 19.6 & 19.8 & 16.8 & 12.5 & $>22 \%$ & 16.4 & 19.3 & 14.0 & 12.6 & 19.6 & 16.2 & 15.1 & 15.1 & 17.3 & 18.9 \\
\hline $\mathrm{B}_{2} \mathrm{O}_{3}$ in glass $(\mathrm{wt} \%)$ & 7.1 & 7.17 & 8.46 & 14.5 & $\sim 5.0$ & 8.4 & 4.66 & 14.39 & 14.19 & 5.6 & 5.48 & 10.5 & 10.5 & 5.94 & 5.6 \\
\hline $\mathrm{Al}_{2} \mathrm{O}_{3}$ in glass (wt $\%$ ) & 4.9 & 4.9 & 5.0 & 6.4 & $>5.0 \%$ & 5.1 & 5.1 & 4.8 & 5.0 & 5.1 & 5.3 & 5.1 & 5.1 & 5.1 & 5.1 \\
\hline
\end{tabular}

Underwashed Case

\begin{tabular}{|c|c|c|c|c|c|c|c|c|c|c|c|c|c|c|c|}
\hline \multicolumn{16}{|l|}{, } \\
\hline WL Range & $25.0-29.5$ & 0 & $23.5-30.0$ & $21.0-28.0$ & 0 & $22.5-30.5$ & $22.5-31.0$ & $23.5-28.5$ & $22.5-30.0$ & $22.5-30.5$ & $25.0-32.0$ & $22.5-30.5$ & $22.5-30.5$ & $23.5-30.5$ & $23.0-30.5$ \\
\hline MAX WL & 29.5 & Restricted & 30 & 28 & Restricted & 30.5 & 31 & 28.5 & 30 & 30.5 & 32 & 30.5 & 30.5 & 30.5 & 30.5 \\
\hline Viscosity (Poise) & 25.5 & & 46.7 & 37.5 & & 30.6 & 27.5 & 37.3 & 34.5 & 30.2 & 75.6 & 54.1 & 39.5 & 44.2 & 33.2 \\
\hline $\mathrm{T}_{\mathrm{L}}\left({ }^{\circ} \mathrm{C}\right)$ & 1024.3 & & 1023 & 1019.8 & & 1020.1 & 1021.6 & 1020.3 & 1023.6 & 1021.9 & 1022.5 & 1023.5 & 1023.5 & 1019.1 & 1021.9 \\
\hline$\Delta \mathrm{G}_{\mathrm{P}}$ & -12.54 & & -10.52 & -6.88 & & -9.62 & -11.42 & -8.72 & -7.39 & -12.05 & -8.96 & -9.01 & -8.9 & -10.21 & -11.57 \\
\hline Homo & 225.7 & & 226.9 & 231.2 & & 230.3 & 231.4 & 223.4 & 229.2 & 230.3 & 233.7 & 230.3 & 230.3 & 228.1 & 229.2 \\
\hline Alkali (wt\%) & 20.2 & $>20.0 \%$ & 17.4 & 13.1 & $>22 \%$ & 17 & 19.8 & 14.5 & 13.2 & 20.2 & 16.8 & 15.7 & 15.7 & 17.9 & 19.5 \\
\hline $\mathrm{B}_{2} \mathrm{O}_{3}$ in glass (wt $\left.\%\right)$ & 7.05 & $\sim 7.5$ & 8.4 & 14.49 & $\sim 5.0$ & 8.40 & 4.63 & 14.39 & 14.09 & 5.56 & 5.44 & 10.43 & 10.43 & 5.94 & 5.56 \\
\hline $\mathrm{Al}_{2} \mathrm{O}_{3}$ in glass (wt $\%$ ) & 4.8 & $\sim 4.5$ & 4.9 & 6.3 & $>5.0 \%$ & 5.0 & 5.1 & 4.7 & 4.9 & 5.0 & 5.3 & 5.0 & 5.0 & 5.0 & 5.0 \\
\hline
\end{tabular}

12 See Brown and Postles (1996) for a more detailed description of the property predictions. 
Table 10. Candidate Frit Compositions (wt\%) and Estimated Properties at a Fixed Waste Loading (25.5 wt\%) For Nominal and Underwashed MB3 Sludge.

\begin{tabular}{|c|c|c|c|c|c|c|c|c|c|c|c|c|c|c|c|}
\hline Oxide & Frit 165 & $\begin{array}{c}\text { Frit } 165 \\
\text { w/o } \mathrm{ZrO}_{2} \\
\end{array}$ & Frit 200 & Frit 303 & Frit 304 & Frit 307 & Frit 313 & Frit 314 & Frit 315 & Frit 320 & Frit 322 & Frit 323 & Frit 324 & Frit 325 & Frit 326 \\
\hline $\mathrm{Al}_{2} \mathrm{O}_{3}$ & 0.00 & 0.00 & 0.00 & 2.29 & 2.29 & 0.00 & 0.00 & 0.00 & 0.00 & 0.00 & 0.00 & 0.00 & 0.00 & 0.00 & 0.00 \\
\hline $\mathrm{B}_{2} \mathrm{O}_{3}$ & 10.00 & 10.10 & 12.00 & 20.13 & 6.71 & 12.08 & 6.71 & 20.13 & 20.13 & 8.00 & 8.00 & 15.00 & 15.00 & 8.55 & 8.00 \\
\hline $\mathrm{Li}_{2} \mathrm{O}$ & 7.00 & 7.07 & 5.00 & 10.12 & 5.80 & 10.74 & 10.74 & 5.94 & 10.03 & 8.00 & 5.00 & 5.19 & 8.28 & 7.55 & 8.00 \\
\hline $\mathrm{Na}_{2} \mathrm{O}$ & 13.00 & 13.13 & 11.00 & 0.00 & 18.07 & 4.67 & 8.75 & 6.12 & 0.00 & 12.00 & 10.00 & 8.28 & 5.19 & 9.10 & 11.00 \\
\hline $\mathrm{SiO}_{2}$ & 68.00 & 68.69 & 70.00 & 67.46 & 67.13 & 72.51 & 73.80 & 65.79 & 69.84 & 72.00 & 77.00 & 71.53 & 71.53 & 72.80 & 72.00 \\
\hline $\mathrm{ZrO}_{2}$ & 1.00 & 0.00 & 0.00 & 0.00 & 0.00 & 0.00 & 0.00 & 2.01 & 0.00 & 0.00 & 0.00 & 0.00 & 0.00 & 0.00 & 0.00 \\
\hline $\mathrm{MgO}$ & 1.00 & 1.01 & 2.00 & 0.00 & 0.00 & 0.00 & 0.00 & 0.00 & 0.00 & 0.00 & 0.00 & 0.00 & 0.00 & 1.50 & 1.00 \\
\hline \multicolumn{16}{|l|}{ Nominal Wash Case } \\
\hline WL Range & $23.0-29.0$ & $22.5-29.0$ & $23.0-29.5$ & $21.0-28.0$ & 0 & $22.0-30.5$ & $22.0-30.5$ & $23.0-28.5$ & $22.0-29.5$ & $22.0-30.0$ & $26.5-31.5$ & $22.0-30.0$ & $22.0-30.0$ & $23.0-30.5$ & $22.5-30.0$ \\
\hline $\mathrm{WL}=25.5$ & 25.5 & 25.5 & 25.5 & 25.5 & 25.5 & 25.5 & 25.5 & 25.5 & 25.5 & 25.5 & 25.5 & 25.5 & 25.5 & 25.5 & 25.5 \\
\hline Viscosity (Poise) & 33.1 & 33.4 & 61.9 & 45.2 & 32.4 & 41.1 & 37.6 & 46.4 & 45.6 & 40.7 & 107.6 & 73.1 & 53.3 & 59.3 & 44.7 \\
\hline $\mathrm{T}_{\mathrm{L}}\left({ }^{\circ} \mathrm{C}\right)$ & 982.7 & 980.7 & 977.0 & 994.2 & 995.3 & 970.4 & 967.2 & 989.4 & 977.5 & 971.7 & 959.7 & 972.9 & 972.9 & 969.6 & 971.7 \\
\hline$\Delta \mathrm{G}_{\mathrm{P}}$ & -12.25 & -12.45 & -10.12 & -6.33 & -13.79 & -9.16 & -11.1 & -8.23 & -6.79 & -11.77 & -8.44 & -8.51 & -8.39 & -9.80 & -11.25 \\
\hline Homo & 217.3 & 218.4 & 217.3 & 226.6 & 226.6 & 219.7 & 219.7 & 217.3 & 219.7 & 219.7 & 219.7 & 219.7 & 219.7 & 217.3 & 218.5 \\
\hline Alkali (wt\%) & 19.7 & 19.8 & 16.7 & 12.3 & 22.6 & 16.3 & 19.3 & 13.8 & 12.3 & 19.7 & 16.0 & 14.8 & 14.8 & 17.2 & 18.9 \\
\hline $\mathrm{B}_{2} \mathrm{O}_{3}$ in glass (wt $\%$ ) & 7.45 & 7.525 & 8.94 & 14.997 & 4.999 & 9 & 4.999 & 14.997 & 14.997 & 5.96 & 5.96 & 11.175 & 11.175 & 6.37 & 5.96 \\
\hline $\mathrm{Al}_{2} \mathrm{O}_{3}$ in glass (wt $\%$ ) & 4.3 & 4.3 & 4.3 & 6.0 & 6.0 & 4.3 & 4.3 & 4.3 & 4.3 & 4.3 & 4.3 & 4.3 & 4.3 & 4.3 & 4.3 \\
\hline \multicolumn{16}{|l|}{ Underwashed Case } \\
\hline WL Range & $25.0-29.5$ & 0 & $23.5-30.0$ & $21.0-28.0$ & 0 & $22.5-30.5$ & $22.5-31.0$ & $23.5-28.5$ & $22.5-30.0$ & $22.5-30.5$ & $25.0-32.0$ & $22.5-30.5$ & $22.5-30.5$ & $25.5-29.5$ & $23.0-30.5$ \\
\hline $\mathrm{WL}=25.5$ & 25.5 & 25.5 & 25.5 & 25.5 & 25.5 & 25.5 & 25.5 & 25.5 & 25.5 & 25.5 & 25.5 & 25.5 & 25.5 & 25.5 & 25.5 \\
\hline Viscosity (Poise) & 31.1 & 31.4 & 58.1 & 42.4 & 30.3 & 38.7 & 35.5 & 43.4 & 42.8 & 38.3 & 101.6 & 68.7 & 50.1 & 55.8 & 42.1 \\
\hline $\mathrm{T}_{\mathrm{L}}\left({ }^{\circ} \mathrm{C}\right)$ & 979.6 & 977.6 & 974 & 990.8 & 991.8 & 967.5 & 964.3 & 986.1 & 974.4 & 968.7 & 957 & 969.9 & 969.9 & 966.7 & 968.7 \\
\hline$\Delta \mathrm{G}_{\mathrm{P}}$ & -12.69 & -12.90 & -10.57 & -6.78 & -14.24 & -9.61 & -11.55 & -8.68 & -7.24 & -12.22 & -8.89 & -8.96 & -8.84 & -10.25 & -11.7 \\
\hline Homo & 216.4 & 217.6 & 216.4 & 225.7 & 225.7 & 218.8 & 218.8 & 216.4 & 218.8 & 218.8 & 218.8 & 218.8 & 218.8 & 216.4 & 217.6 \\
\hline Alkali (wt\%) & 20.2 & 20.3 & 17.2 & 12.8 & 23 & 16.7 & 19.8 & 14.2 & 12.7 & 20.2 & 16.4 & 15.3 & 15.3 & 17.7 & 19.4 \\
\hline $\mathrm{B}_{2} \mathrm{O}_{3}$ in glass (wt $\%$ ) & 7.45 & 7.525 & 8.94 & 14.997 & 4.999 & 9 & 4.999 & 14.997 & 14.997 & 5.96 & 5.96 & 11.175 & 11.175 & 6.37 & 5.96 \\
\hline $\mathrm{Al}_{2} \mathrm{O}_{3}$ in glass (wt $\%$ ) & 4.2 & 4.2 & 4.2 & 5.9 & \begin{tabular}{l|l}
5.9 \\
\end{tabular} & 4.2 & 4.2 & 4.2 & 4.2 & 4.2 & 4.2 & 4.2 & 4.2 & 4.3 & 4.2 \\
\hline
\end{tabular}


Table 11. PAR Limits for Various Properties.

\begin{tabular}{|l|c|}
\hline \hline Property & PAR Limit \\
\hline \hline $\mathrm{T}_{\mathrm{L}}$ & $<1024.95^{\circ} \mathrm{C}$ \\
\hline Homogeneity & $>210.92$ \\
\hline$\Delta \mathrm{G}_{\mathrm{P}}$ (durability) & $>-12.7178$ \\
\hline$\eta_{1150^{\circ} \mathrm{C}}$ & $21.5-105.4$ Poise \\
\hline
\end{tabular}

\subsubsection{Nominal and Underwashed Scenarios at Maximum Waste Loadings}

From Table 9, all proposed frit compositions, with the exception of Frit 304, yield relatively wide operating windows based on PCCS model predictions. Maximum waste loadings for each of the frits (again excluding Frit 304) range from $28.0 \mathrm{wt} \%$ (Frit 303) to $31.5 \mathrm{wt} \%$ (Frit 322). Current PCCS model predictions will not allow Frit 304 to be processed. This is a result of the predicted $\Delta \mathrm{G}_{\mathrm{P}}$ (durability) values being less than the PAR limit $(-12.7178 \mathrm{kcal} / 100 \mathrm{~g}$ glass $)$ and is an issue that will be discussed in detail in Sections 7.2 and 8.0.

Numerous comparisons could be made given the property predictions listed in Table 9. In this report, a brief description of those that the authors feel are critical to meeting the objectives of this overall task will be discussed. For example, MB3N320q (MB3, nominal wash, Frit 320, quenched) at $30.0 \mathrm{wt} \%$ loading has a predicted $\eta_{1150^{\circ} \mathrm{C}}, \mathrm{T}_{\mathrm{L}}$, and $\Delta \mathrm{G}_{\mathrm{P}}$ of 33.5 Poise, $1020^{\circ} \mathrm{C}$, and -11.54 , respectively. This particular glass appears to be $T_{L}$ limited $\left(P A R\right.$ limit $\left.=1024.95^{\circ} \mathrm{C}\right)$ in terms of waste loading. MB3U320q $(\mathrm{MB} 3$, underwashed, Frit 320, quenched) is predicted to have a slightly higher waste loading compared to its nominal washed counterpart (30.5 wt \% versus $30.0 \mathrm{wt} \%$ ). At 30.5\% loading, the underwashed Frit 320 glass still appears $\mathrm{T}_{\mathrm{L}}$ limited. As expected, the predicted impact of the underwashed sludge (increased $\mathrm{Na}_{2} \mathrm{O}$ content) is on durability. The $\Delta \mathrm{G}_{\mathrm{P}}$ for MB3U320q is -12.05 which is approaching the -12.7178 PAR limit listed in Table 11. If the hypothesis holds true that increasing the total alkali content in the glass should improve melt rate, glass formulation efforts will strive to maximize the sum of alkali while maintaining acceptable property predictions or actual measurements. 
The "baseline" frit (Frit 200) does yield a processing window with both the nominal and underwashed MB3 sludge. Projected maximum waste loadings range up to 29.5 and $30.0 \mathrm{wt} \%$ for the nominal and underwashed scenarios, respectively. For Frit 200, $\eta$ seems to be the property that stands out relative to the majority of other candidate frits. For comparison, if lowering $\eta$ enhances melt rate, the $\eta_{1150^{\circ} \mathrm{C}}$ for MB3N200 and MB3U200 are 51.8 and 46.7 Poise respectively. Although not as high as that predicted for glasses produced from Frit 322, Frit 323, Frit 324, or Frit 325, the relatively high viscosities of MB3N200 and MB3U200 may slow down melt rate relative to other candidate frits (e.g., Frit 165, Frit 304 or Frit 320). It should be noted that the $\eta$ values shown in Tables 9 and 10 are predicted glass viscosities at $1150^{\circ} \mathrm{C}$, not the $\eta$ of the liquid phase critical to foam formation. The $\eta$ of the liquid phase critical to foam formation will likely be the result of an interaction between a molten salt (e.g., $\mathrm{NaNO}_{3}$ or $\mathrm{NaNO}_{2}$ ) and the frit. The $\eta$ of the glass does provide insight into the potential for convection currents in the molten glass pool to sweep the foamy layer (if it exists) from the batch-melt interface, which would accelerate melt rates. A low- $\eta$ glass should increase convection rates resulting in a heat transfer rate increase to the bottom of the cold cap. Therefore, a lower $\eta$ (but still within the PAR limits) is being considered a positive influence on melt rate as candidate frits are assessed.

The use of Frit 165 without $\mathrm{ZrO}_{2}$ is a very interesting case. With the nominally washed sludge, PCCS predictions (at the PAR) provide an operational window from $22.5-29.0 \mathrm{wt} \%$. At the maximum waste loading, the system appears to be either $T_{L}$ or durability limited with predicted values of $T_{L}=1019.8^{\circ} \mathrm{C}$ and a $\Delta \mathrm{G}_{\mathrm{P}}=-12.24$. If Frit 165 without $\mathrm{ZrO}_{2}$ was selected as the primary candidate for $\mathrm{MB} 3$, based on the PCCS calculations (again these initial model assessment calculations excluded some minor components), there would be no operating window for the underwashed MB3 scenario. The system is durability limited with $\Delta \mathrm{G}_{\mathrm{P}}$ 's on the order of -12.90 at $25.5 \% \mathrm{WL}$ (not meeting the PAR limit). Given that uncertainties in washing efficiencies, analysis and loadings have not been considered, it would appear that the use of Frit 165 without $\mathrm{ZrO}_{2}$ for MB3 would be questionable or on the "borderline of unacceptability".13

The use of Frit 165 (with $\mathrm{ZrO}_{2}$ ) does provide operational windows for both the nominal and underwashed MB3 scenarios. Based on $\Delta \mathrm{G}_{\mathrm{P}}$ and $\mathrm{T}_{\mathrm{L}}$ predictions, this system again appears to be either durability or $\mathrm{T}_{\mathrm{L}}$ limited in terms of waste loadings.

13 It should be noted that this discussion is based on the removal of $1 \mathrm{wt} \% \mathrm{ZrO}_{2}$ and renormalizing the remaining frit components. Other options exists for reformulating a non- $\mathrm{ZrO}_{2}$ based Frit 165 that may lead to a predictable operational window. 
Although the initial screening process considered compositional limits (e.g., $\mathrm{B}_{2} \mathrm{O}_{3}$ contents $<11-12$ wt $\%$ in glass), the impact of these higher $\mathrm{B}_{2} \mathrm{O}_{3}$ glasses on predicted properties does not appear to be a concern. This may be the result of the data from which the models were developed did not contain high $\mathrm{B}_{2} \mathrm{O}_{3}$ glasses. Consider MB3N303q which has $14.9 \% \mathrm{~B}_{2} \mathrm{O}_{3}$ in glass $(\mathrm{WL}=28 \mathrm{wt} \%)$ but still yields a predicted $\Delta \mathrm{G}_{\mathrm{P}}$ of -6.39 and a homogeneity calculation of 232.1 . Both predicted values are well within the PAR limits listed in Table 11.

Again, it should be noted that the objective of this study was to improve melt rate and the predictions are only intended to help guide our thought process. General trends indicate that over the frit compositional range evaluated the impact of an underwashed MB3 sludge (for a given frit composition and WL) is that both durability and $\mathrm{T}_{\mathrm{L}}$ are slightly reduced. If total alkali content does enhance melt rate, one would hope to be in a position of making the glass "durability limited" instead of $\mathrm{T}_{\mathrm{L}}$ limited.

\subsubsection{Nominal and Underwashed Scenarios at Fixed Waste Loading}

Numerous comparisons could also be made given the property predictions listed in Table 10. In this report, a brief description of those that the authors feel are critical to meeting the objectives of this overall task will be discussed. By fixing the WL one can make direct comparisons between frit compositions and their effect on predicted properties. The operational window or WL range for Frit 322 is 26.5 - 31.5 wt $\%$. Therefore, the properties shown at 25.5 wt $\%$ are "invalid" for Frit 322 in terms of acceptability per current PCCS model predictions (fails PAR $\eta$ criteria as shown in Table 11). Even though properties are shown for Frit 304, this system is predicted to be durability limited over the entire range of waste loadings evaluated $(21.0-36.0 \mathrm{wt} \%)$ for both nominal and underwashed scenarios.

\subsection{EXPERIMENTAL}

In this section, we describe a limited number of tests that were performed on critical properties of interest in an effort to validate model predictions and/or reduce technical risks. The experimental procedures, test equipment, and application of standards used to generate the required data in support of the test objective(s) are discussed. It should be noted that models to directly assess melt rate do not exist and that the models being utilized to assess specific properties may not have been developed over the projected compositional range of interest for all possible compositional combinations of candidate frit(s) and MB3 mixtures. 


\subsection{GLASS FABRicATION}

Table 12 identifies twenty-six uranium-based MB3 glasses prepared (at a fixed waste loading of 25.5\%) and tested in this study. Thirteen glasses were fabricated based on the nominal MB3 sludge composition. The other thirteen glasses were based on the underwashed MB3 composition. The primary objective was to measure the effect of frit composition on durability (as defined by the PCT) for both nominal and underwashed sludge cases. In an effort to address the potential impacts of thermal history on durability, both quenched and centerline canister cooled (clc) glasses were tested.

Each batch was prepared to produce 100 grams of glass ${ }^{14}$ from the proper proportions of reagent grade chemicals using SRTC technical procedure "Glass Batch Preparation Procedure - GTOP-3-003" (SRTC 1996a). Weigh sheets were filled out as the materials were weighed. Once batched, these glasses were melted in accordance with the technical procedure "Glass Melting Procedure - GTOP-3-004" (SRTC 1996b). In general, the raw materials were thoroughly mixed and placed into a 95\% Platinum / 5\% Gold $250 \mathrm{ml}$ crucible. The batch was subsequently placed into a high temperature furnace and the temperature was increased at $\sim 10^{\circ} \mathrm{C} /$ minute to $1150^{\circ} \mathrm{C}$. After an isothermal hold at $1150^{\circ} \mathrm{C}$ for $4 \mathrm{~h}$, the crucible was removed and the glass was poured onto a clean stainless steel plate and allowed to air cool. It should be noted that these glasses were not fabricated using the dried melter feed prepared by Stone and Lambert (2001). The objective of this study was to assess property - composition relationships; not to assess melt rate behavior.

Approximately 90 grams of glass were removed (poured) from the crucible while $\sim 10$ grams remained in the crucible along the walls. 15 The pour patty was used as a sampling stock for the various heat treatments and property measurements (i.e., chemical composition, durability, and $\eta$ ). Glasses were stored in marked containers (using unique nomenclatures (see Appendix A)).

To bound the effects of thermal history on the product performance, approximately 25 grams of each MB3 glass was heat treated to simulate cooling along the centerline of a DWPF-type canister (Marra and Jantzen 1993). This cooling regime is commonly referred to as the centerline canister cooling (clc) curve. This terminology will be utilized in this report to differentiate samples from different cooling regimes (quenched versus clc).

14 This would produce enough glass from which all processing and product performance properties could be measured.

15 Visual observations of homogeneity were documented in WSRC-NB-99-00237 for both the pour patty and the residual crucible glass. No visual signs of undissolved solids or compositional inhomogeneities were observed. 
Table 12. Targeted Oxide Compositions (in weight percents, wt\%'s) of the MR Glasses.

\begin{tabular}{|c|c|c|c|c|c|c|c|c|c|c|c|c|c|}
\hline \multirow{2}{*}{$\begin{array}{c}\text { Glass } \\
\text { Identifiers }\end{array}$} & \multirow{2}{*}{$\begin{array}{l}\text { Glass ID's } \\
\text { (shortened) }\end{array}$} & \multicolumn{12}{|c|}{ Oxide } \\
\hline & & $\mathrm{Al}_{2} \mathrm{O}_{3}$ & $\mathbf{B}_{2} \mathbf{O}_{3}$ & $\mathrm{CaO}$ & $\mathrm{Fe}_{2} \mathrm{O}_{3}$ & $\mathbf{L i}_{2} \mathbf{O}$ & MgO & $\mathrm{MnO}$ & $\mathrm{Na}_{2} \mathrm{O}$ & $\mathrm{NiO}$ & $\mathrm{SiO}_{2}$ & $\mathrm{U}_{3} \mathrm{O}_{8}$ & $\mathrm{ZrO}_{2}$ \\
\hline MB3N165 & N165 & 4.296 & 7.450 & 0.954 & 10.987 & 5.215 & 0.806 & 0.720 & 14.464 & 0.491 & 51.175 & 2.697 & 0.745 \\
\hline MB3N200 & N200 & 4.296 & 8.940 & 0.954 & 10.987 & 3.725 & 1.551 & 0.720 & 12.974 & 0.491 & 52.665 & 2.697 & 0.000 \\
\hline MB3N303 & N303 & 6.002 & 14.997 & 0.954 & 10.987 & 7.539 & 0.061 & 0.720 & 4.779 & 0.491 & 50.772 & 2.697 & 0.000 \\
\hline MB3N304 & N304 & 6.002 & 4.999 & 0.954 & 10.987 & 4.321 & 0.061 & 0.720 & 18.241 & 0.491 & 50.526 & 2.697 & 0.000 \\
\hline MB3N307 & N307 & 4.296 & 9.000 & 0.954 & 10.987 & 8.001 & 0.061 & 0.720 & 8.258 & 0.491 & 54.535 & 2.697 & 0.000 \\
\hline MB3N313 & N313 & 4.296 & 4.999 & 0.954 & 10.987 & 8.001 & 0.061 & 0.720 & 11.298 & 0.491 & 55.496 & 2.697 & 0.000 \\
\hline MB3N314 & N314 & 4.296 & 14.997 & 0.954 & 10.987 & 4.425 & 0.061 & 0.720 & 9.338 & 0.491 & 49.536 & 2.697 & 1.497 \\
\hline MB3N315 & N315 & 4.296 & 14.997 & 0.954 & 10.987 & 7.472 & 0.061 & 0.720 & 4.779 & 0.491 & 52.545 & 2.697 & 0.000 \\
\hline MB3N320 & N320 & 4.296 & 5.960 & 0.954 & 10.987 & 5.960 & 0.061 & 0.720 & 13.719 & 0.491 & 54.155 & 2.697 & 0.000 \\
\hline MB3N322 & N322 & 4.296 & 5.960 & 0.954 & 10.987 & 3.725 & 0.061 & 0.720 & 12.229 & 0.491 & 57.880 & 2.697 & 0.000 \\
\hline MB3N323 & N323 & 4.296 & 11.175 & 0.954 & 10.987 & 3.867 & 0.061 & 0.720 & 10.947 & 0.491 & 53.804 & 2.697 & 0.000 \\
\hline MB3N324 & N324 & 4.296 & 11.175 & 0.954 & 10.987 & 6.169 & 0.061 & 0.720 & 8.645 & 0.491 & 53.804 & 2.697 & 0.000 \\
\hline MB3N326 & N326 & 4.296 & 5.960 & 0.954 & 10.987 & 5.960 & 0.806 & 0.720 & 12.974 & 0.491 & 54.155 & 2.697 & 0.000 \\
\hline MB3U165 & U165 & 4.192 & 7.450 & 0.941 & 10.824 & 5.215 & 0.806 & 0.691 & 14.944 & 0.460 & 51.168 & 2.563 & 0.745 \\
\hline MB3U200 & U200 & 4.192 & 8.940 & 0.941 & 10.824 & 3.725 & 1.551 & 0.691 & 13.454 & 0.460 & 52.658 & 2.563 & 0.000 \\
\hline MB3U303 & U303 & 5.898 & 14.997 & 0.941 & 10.824 & 7.539 & 0.061 & 0.691 & 5.259 & 0.460 & 50.766 & 2.563 & 0.000 \\
\hline MB3U304 & U304 & 5.898 & 4.999 & 0.941 & 10.824 & 4.321 & 0.061 & 0.691 & 18.721 & 0.460 & 50.520 & 2.563 & 0.000 \\
\hline MB3U307 & U307 & 4.192 & 9.000 & 0.941 & 10.824 & 8.001 & 0.061 & 0.691 & \begin{tabular}{|l|}
8.738 \\
\end{tabular} & 0.460 & 54.528 & 2.563 & 0.000 \\
\hline MB3U313 & U313 & 4.192 & 4.999 & 0.941 & 10.824 & 8.001 & 0.061 & 0.691 & 11.778 & 0.460 & 55.489 & 2.563 & 0.000 \\
\hline MB3U314 & U314 & \begin{tabular}{|l|}
4.192 \\
\end{tabular} & 14.997 & 0.941 & 10.824 & 4.425 & 0.061 & 0.691 & 9.819 & 0.460 & 49.529 & 2.563 & 1.497 \\
\hline MB3U315 & U315 & 4.192 & 14.997 & 0.941 & 10.824 & 7.472 & 0.061 & 0.691 & 5.259 & 0.460 & 52.539 & 2.563 & 0.000 \\
\hline MB3U320 & U320 & 4.192 & 5.960 & 0.941 & 10.824 & 5.960 & 0.061 & 0.691 & 14.199 & 0.460 & 54.148 & 2.563 & 0.000 \\
\hline MB3U322 & U322 & \begin{tabular}{|l|}
4.192 \\
\end{tabular} & 5.960 & 0.941 & 10.824 & 3.725 & 0.061 & \begin{tabular}{|l|}
0.691 \\
\end{tabular} & 12.709 & 0.460 & 57.873 & 2.563 & 0.000 \\
\hline MB3U323 & U323 & 4.192 & 11.175 & 0.941 & 10.824 & 3.867 & 0.061 & 0.691 & 11.428 & 0.460 & 53.798 & 2.563 & 0.000 \\
\hline MB3U324 & U324 & 4.192 & 11.175 & 0.941 & 10.824 & 6.169 & 0.061 & 0.691 & \begin{tabular}{|l|}
9.126 \\
\end{tabular} & 0.460 & 53.798 & 2.563 & 0.000 \\
\hline MB3U326 & U326 & 4.192 & 5.960 & 0.941 & 10.824 & 5.960 & 0.806 & 0.691 & 13.454 & 0.460 & 54.148 & 2.563 & 0.000 \\
\hline
\end{tabular}

\subsection{Property Measurements}

\subsubsection{ChEMiCAL COMPOSITION ANALYSIS}

To confirm that the as-fabricated glasses corresponded to the target compositions (as shown in Table 12), a representative sample from each as-fabricated MB3 glass was submitted to the SRTC Mobile Laboratory (SRTC-ML) for chemical analysis. Edwards provided an analytical plan (see Appendix B) that accompanied these samples. This plan identified the elements to be analyzed and the dissolution techniques (i.e., sodium peroxide fusion [PF] or lithium-metaborate [LM] flux) to be used. Each glass was prepared in duplicate by each of the dissolution techniques. Concentrations (as weight \%) for the following elements were measured by Inductively Coupled Plasma - Atomic Emission Spectroscopy (ICP - AES): aluminum, boron, calcium, chromium, iron, lithium, magnesium, manganese, nickel, sodium, silicon, uranium, and zirconium. The analytical plan was developed in such a way as to provide the opportunity to evaluate potential sources of error. The results were evaluated to confirm that the 
target glass compositions were adequately met. Standard glasses (including the Wasteform Compliance Plan (WCP) Batch 1 standard and a Corning Engineering Laboratory Services [CELS] uraniumcontaining glass $\left[\mathrm{U}_{\mathrm{std}}\right]$ ) were intermittently run to assess the performance of the ICP over the course of these analyses and to determine potential bias-correction $(\mathrm{bc})$ needs.

\subsubsection{DURABILITY}

The Product Consistency Test (PCT) was performed on each glass to assess chemical durability using technical procedure "Nuclear Waste Glass Product Consistency Test (PCT) Method - GTOP-3-025" (SRTC 1998) which is compliant with the ASTM C1285-97 (ASTM 1997). The PCT was conducted in triplicate for each MB3 glass (both quenched and clc versions). Also included in this experimental test matrix were the Environmental Assessment (EA) glass (Jantzen et al. 1993), the Approved Reference Material (ARM-1) glass, and blanks. Samples were ground, washed, and prepared according to procedure. Fifteen (15) $\mathrm{ml}$ of Type I ASTM water were added to 1.5 grams of glass in stainless steel vessels. The vessels were closed, sealed, and placed in an oven at $90 \pm 2^{\circ} \mathrm{C}$. Samples were left at $90^{\circ} \mathrm{C} \pm$ $2{ }^{\circ} \mathrm{C}$ for 7 days. The resulting solutions (once cooled) were sampled (filtered and acidified), labeled (according to the analytical plan), and analyzed. Edwards provided analytical plans for the solution analysis (see Appendices C and D - due to the large number of vessels, two sets of tests were initiated).16 The overall philosophy of these plans was to provide an opportunity to assess the consistency (repeatability) of the PCT and analytical procedures in the effort to evaluate chemical durability of the MB3 glasses. Normalized releases were calculated based on target, measured and bias-corrected compositions using the average of the logs.

\subsubsection{VISCOSITY}

High temperature $\eta$ was measured as a function of temperature (T) using a spindle viscometer for selected MB3 glasses. The measurements were obtained using Glass Technology Operating Procedure (GTOP) 3111 "Determination of Glass Viscosity" (SRTC 1999 and Schumacher and Peeler 1998). High temperature $\eta$ data were measured over the maximum temperature range allowable for each glass. The low temperature limit was based on the effects of crystallization on the melt pool. The high temperature limit was based on limiting the effects of volatilization. To validate the glass- $\eta$ data, the $\eta$ of the Batch 1

\footnotetext{
16 Due to the number of glasses to be tested ( 52 glasses as a result of 26 glasses with two heat treatments - not including blanks and standards), PCT's were performed in two sets. Set \#1 contained all the nominal washed MB3 glasses (both quenched and clc. Set \#2 contained all the underwashed MB3 glasses (both quenched and clc). It should be noted that blanks and standards were run in both sets. See Appendix C and Appendix D for more details.
} 
standard glass (Schumacher and Peeler 1998) was measured at the beginning and end of this study. Viscosity at $1150^{\circ} \mathrm{C}\left(\eta_{1150^{\circ} \mathrm{C}}\right)$ for each glass was predicted from a Fulcher fit of the measured data.

\subsection{RESULTS AND DISCUSSION}

This section provides a detailed discussion and analysis of the measured MB3 glass compositions, homogeneity evaluation via visual observations, PCT results and $\eta$ results.

\subsection{CHEMICAL COMPOSITION ANALYSIS}

The measured chemical compositions of the MB3 glasses are presented and reviewed in this section. Comparisons are made between measured and targeted compositions. Measurements of standards that were analyzed along with the study glasses are also presented. The results from the standards were used to bias correct the measurements for the study glasses (when possible). This approach provides an additional view of the chemical compositions of the study glasses for consideration and interpretation. The statistical review was conducted using JMP® Version 4.0, a commercially available software package from SAS Institute, Inc. (SAS 2000).

Table 12 provides the targeted oxide compositions and the glass identifiers for each of the MB3 study glasses. In some instances, shortened versions (also in Table 12) of the identifiers are used in the discussion that follows.

In addition to the study glasses, a standard glass (WCP Batch 1) and an uranium-bearing glass $\left(\mathrm{U}_{\text {std }}\right)$ were included in the planning of the analyses (for possible bc). An analytical plan (in the form of a memorandum) was provided to assist the SRTC-Mobile Laboratory (SRTC-ML) in conducting these analyses (see Appendix B).

Tables E1 and E2 in Appendix E provide the composition measurements obtained by the SRTC-ML for the analytical plan given in Appendix B. Table E1 provides the measurements generated from lithium metaborate preparations. Table E2 presents the measurements generated from the peroxide fusion preparations. The measurements of Tables E1 and E2 are provided in elemental weight percents (wt\%'s). Values below the detection limit of the procedures are indicated by a "<" symbol followed by the detection limit. These elemental concentrations were replaced by $1 / 2$ of the detection limits in this report. The elemental concentrations were converted to oxide concentrations by multiplying by the appropriate 
gravimetric factors, and in the discussion that follows the chemical compositions of the glasses are presented as oxides. The measurements were completed by the use of ICP-AES.

Exhibit E1 in Appendix E provides plots of the oxide concentration measurements (as wt\%'s) in ICP analytical sequence for the samples prepared via LM, and Exhibit E2 in Appendix E provides similar plots for the samples prepared using PF.

A review of the results from the standards provides insight into the possibility that the ICP calibration contributes (in a systematic way) to the oxide measurements for the study glasses. Exhibit E3 in Appendix E provides plots of the Batch 1 and $\mathrm{U}_{\text {std }}$ oxide measurements per analytical block by oxide for the LM results, and Exhibit E4 in Appendix E provides the same type of plots for the PF results. For many of these oxides, the Batch 1 results indicate statistically significant differences among the block averages. The behavior of the $\mathrm{U}_{\text {std }}$ values for an oxide follows that of the Batch 1 measurements as well. These results suggest that bias correcting for ICP calibration effects may be advantageous.

Table 13 provides the average measured composition by analytical block for the two glass standards included in the analytical plan. It also provides the reference values for the standards.

The analytical results from the Batch 1 samples are to be used to bias correct for a possible ICP calibration effect (a block effect) in the other measurements. This is accomplished for each oxide in turn by taking the original oxide measurement, noting its block, and then multiplying the measurement by the ratio of the corresponding reference value for Batch 1 divided by the average oxide measurement for Batch 1 in that block. This approach was used to bias correct the composition measurements of the MB3 and both standard glasses for all oxides except $\mathrm{U}_{3} \mathrm{O}_{8}$. The $\mathrm{U}_{\text {std }}$ results were used to bias correct only the uranium numbers for the study glasses and for Batch 1. 
Table 13. Average Measurements by Analytical Block for Glass Standards.

\begin{tabular}{|c|c|c|c|c|c|c|c|c|c|}
\hline \multirow{2}{*}{$\begin{array}{l}\text { Batch 1 } \\
\text { Oxide }\end{array}$} & $1-1$ & $1-2$ & $2-1$ & $2-2$ & 3-1 & $3-2$ & 4-1 & $4-2$ & \multirow{2}{*}{$\begin{array}{c}\text { Reference } \\
\text { Value }\end{array}$} \\
\hline & 3 obs & 3 obs & 3 obs & 3 obs & 3 obs & 3 obs & 3 obs & 3 obs & \\
\hline $\mathrm{Al}_{2} \mathrm{O}_{3}$ & 4.831 & 4.875 & 4.837 & 4.919 & 4.881 & 4.888 & 4.799 & 4.799 & 4.877 \\
\hline $\mathrm{B}_{2} \mathrm{O}_{3}$ & 8.179 & 8.093 & 7.824 & 7.964 & 7.910 & 8.039 & 7.910 & 7.803 & 7.777 \\
\hline $\mathrm{CaO}$ & 1.278 & 1.292 & 1.299 & 1.251 & 1.294 & 1.285 & 1.290 & 1.289 & 1.220 \\
\hline $\mathrm{Cr}_{2} \mathrm{O}_{3}$ & 0.103 & 0.111 & 0.100 & 0.128 & 0.118 & 0.104 & 0.108 & 0.110 & 0.107 \\
\hline $\mathrm{Fe}_{2} \mathrm{O}_{3}$ & 13.339 & 12.939 & 12.834 & 13.072 & 13.067 & 12.434 & 13.449 & 13.501 & 12.839 \\
\hline $\mathrm{Li}_{2} \mathrm{O}$ & 4.385 & 4.421 & 4.421 & 4.485 & 4.449 & 4.500 & 4.406 & 4.392 & 4.429 \\
\hline $\mathrm{MgO}$ & 1.418 & 1.448 & 1.429 & 1.521 & 1.437 & 1.452 & 1.451 & 1.448 & 1.419 \\
\hline $\mathrm{MnO}$ & 1.679 & 1.691 & 1.683 & 1.782 & 1.687 & 1.709 & 1.683 & 1.679 & 1.726 \\
\hline $\mathrm{Na}_{2} \mathrm{O}$ & 8.901 & 9.216 & 9.288 & 9.202 & 8.996 & 9.202 & 9.310 & 9.175 & 9.003 \\
\hline $\mathrm{NiO}$ & 0.709 & 0.720 & 0.715 & 0.753 & 0.717 & 0.720 & 0.718 & 0.716 & 0.751 \\
\hline $\mathrm{SiO}_{2}$ & 51.272 & 50.131 & 49.774 & 50.202 & 50.202 & 48.419 & 50.773 & 51.058 & 50.220 \\
\hline $\mathrm{U}_{3} \mathrm{O}_{8}$ & 0.166 & 0.166 & 0.166 & 0.166 & 0.166 & 0.166 & 0.166 & 0.166 & 0.000 \\
\hline $\mathrm{ZrO}_{2}$ & 0.123 & 0.127 & 0.127 & 0.152 & 0.121 & 0.125 & 0.124 & 0.123 & 0.098 \\
\hline Sum of Oxides & 96.38 & 95.23 & 94.50 & 95.60 & 95.05 & 93.04 & 96.19 & 96.26 & 94.37 \\
\hline $\mathbf{U}_{\text {std }}$ & $1-1$ & $1-2$ & $2-1$ & $2-2$ & 3-1 & $3-2$ & 4-1 & 4-2 & \multirow{2}{*}{$\begin{array}{c}\text { Reference } \\
\text { Value }\end{array}$} \\
\hline Oxide & 2 obs & 2 obs & 2 obs & 2 obs & 2 obs & 2 obs & 2 obs & 2 obs & \\
\hline $\mathrm{Al}_{2} \mathrm{O}_{3}$ & 4.025 & 4.119 & 4.015 & 4.138 & 4.053 & 4.100 & 40.072 & 4.053 & 4.100 \\
\hline $\mathrm{B}_{2} \mathrm{O}_{3}$ & 9.241 & 9.354 & 8.984 & 9.273 & 8.984 & 9.064 & 9.257 & 8.839 & 9.209 \\
\hline $\mathrm{CaO}$ & 1.281 & 1.294 & 1.291 & 1.243 & 1.307 & 1.296 & 1.291 & 1.292 & 1.301 \\
\hline $\mathrm{Cr}_{2} \mathrm{O}_{3}$ & 0.243 & 0.253 & 0.245 & 0.277 & 0.254 & 0.246 & 0.251 & 0.247 & 0.000 \\
\hline $\mathrm{Fe}_{2} \mathrm{O}_{3}$ & 13.311 & 13.218 & 12.967 & 13.232 & 13.246 & 12.117 & 13.704 & 13.589 & 13.196 \\
\hline $\mathrm{Li}_{2} \mathrm{O}$ & 2.960 & 3.014 & 2.993 & 3.036 & 2.993 & 3.046 & 2.982 & 2.949 & 3.057 \\
\hline $\mathrm{MgO}$ & 1.181 & 1.212 & 1.210 & 1.277 & 1.191 & 1.207 & 1.221 & 1.201 & 1.210 \\
\hline $\mathrm{MnO}$ & 2.641 & 2.666 & 2.686 & 2.795 & 2.621 & 2.673 & 2.666 & 2.628 & 2.892 \\
\hline $\mathrm{Na}_{2} \mathrm{O}$ & 11.505 & 11.829 & 11.721 & 11.680 & 11.404 & 12.220 & 11.822 & 11.579 & 11.795 \\
\hline $\mathrm{NiO}$ & 0.991 & 1.007 & 1.010 & 1.046 & 0.989 & 1.008 & 1.005 & \begin{tabular}{|l|}
0.993 \\
\end{tabular} & 1.120 \\
\hline $\mathrm{SiO}_{2}$ & 47.279 & 46.851 & 46.209 & 46.530 & 46.637 & 44.497 & 47.279 & 47.279 & 45.353 \\
\hline $\mathrm{U}_{3} \mathrm{O}_{8}$ & 2.288 & 2.364 & 2.258 & 2.400 & 2.376 & 2.335 & 2.382 & 2.347 & 2.406 \\
\hline $\mathrm{ZrO}_{2}$ & 0.006 & 0.007 & 0.001 & 0.030 & 0.005 & 0.005 & 0.009 & 0.004 & 0.000 \\
\hline Sum of Oxides & 96.95 & 97.19 & 95.59 & 96.96 & 96.06 & 93.81 & 97.94 & 97.00 & 95.64 \\
\hline
\end{tabular}


More specifically, for each dissolution method, let $\bar{a}_{i j}$ be the average measurement for the $i^{\text {th }}$ oxide at analytical block $\mathrm{j}$ for Batch 1 (or $\mathrm{U}_{\text {std }}$ for uranium), and let $\mathrm{t}_{\mathrm{i}}$ be the reference value for the $\mathrm{i}^{\text {th }}$ oxide. Table 13 provides the averages and reference values for each oxide of interest. Let $\bar{c}_{i j k}$ be the average measurement for the $\mathrm{i}^{\text {th }}$ oxide at analytical block $\mathrm{j}$ for the $\mathrm{k}^{\text {th }}$ glass prepared by the given dissolution method. The bias adjustment was conducted as follows for each of the two dissolution methods

$$
\bar{c}_{i j k} \bullet\left(1-\frac{\overline{\mathrm{a}}_{i j}-\mathrm{t}_{\mathrm{i}}}{\overline{\mathrm{a}}_{\mathrm{ij}}}\right)=\overline{\mathrm{c}}_{\mathrm{ijk}} \bullet \frac{\mathrm{t}_{\mathrm{i}}}{\overline{\mathrm{a}}_{\mathrm{ij}}}
$$

Bias corrected measurements are indicated by a "bc" suffix, and such adjustments were performed for all of the oxides and both of the dissolution methods of this study. This approach was used to bias correct the composition measurements of the MB3 study glasses and standard glasses. Both measured and measured "bc" values are included in the discussion that follows.

Exhibits E5 and E6 in Appendix E provide plots of the concentration measurements for each oxide for each of the glasses (including the standards) for the LM and PF preparations, respectively. Both measured and bias-corrected values are plotted. These plots are useful in assessing the repeatability of the measurements for each glass. The most significant observation for these glasses is that several glasses show one exceptionally low $\mathrm{Fe}_{2} \mathrm{O}_{3}$ value in Exhibit E5. Most of these low values appear to have occurred in analytical block 3-2. A look back at Exhibit E3 shows that the measurements for the standards were low for this block as well. Bias correction does not appear to remedy this problem as seen in Exhibit E5.

Table 14 provides summary information for the measurements of the study glasses as well as their targeted values. The sums of oxides for the targeted, measured, and measured bias-corrected (bc) compositions are also provided. A review of these sums shows that they are all within the interval of 95 to 105 weight percent with the smallest value being $98.6 \mathrm{wt} \%$ for the measured, bias-corrected composition of N314 and the largest value being $104.4 \mathrm{wt} \%$ for the measured composition of N323. The measurements and bias-corrected measurements that differ from the targeted values by more than $5 \%$ have been shaded in Table 14 . 
Table 14. Measured, Measured Bias-Corrected (bc), and Targeted Compositions for the MB3 Study Glasses.

\begin{tabular}{|c|c|c|c|c|c|c|c|c|c|c|c|c|c|c|c|c|}
\hline $\begin{array}{c}\text { Glass } \\
\text { ID }\end{array}$ & $\begin{array}{c}\begin{array}{c}\text { Glass ID } \\
\text { (shortened) }\end{array} \\
\end{array}$ & $\begin{array}{c}\text { Compositional } \\
\text { View }\end{array}$ & $\mathrm{Al}_{2} \mathrm{O}_{3}$ & $\mathbf{B}_{2} \mathbf{O}_{3}$ & $\mathrm{CaO}$ & $\mathrm{Cr}_{2} \mathrm{O}_{3}$ & $\mathrm{Fe}_{2} \mathrm{O}_{3}$ & $\mathbf{L i}_{2} \mathbf{O}$ & MgO & MnO & $\mathrm{Na}_{2} \mathrm{O}$ & $\mathrm{NiO}$ & $\mathrm{SiO}_{2}$ & $\mathbf{U}_{3} \mathbf{O}_{8}$ & $\mathrm{ZrO}_{2}$ & $\begin{array}{l}\text { Sum of } \\
\text { Oxides }\end{array}$ \\
\hline MB3N165 & N165 & Measured & 4.469 & 7.285 & 0.964 & 0.019 & 11.277 & 5.135 & 0.845 & 0.748 & 14.828 & 0.485 & 52.466 & 2.388 & 0.692 & 101.600 \\
\hline MB3N165 & N165 & Measured bc & 4.468 & 7.177 & 0.912 & 0.018 & 11.359 & 5.107 & 0.830 & 0.760 & 14.673 & 0.506 & 53.444 & 2.467 & 0.551 & 102.272 \\
\hline MB3N165 & N165 & Targeted & 4.296 & 7.450 & 0.954 & 0.000 & 10.987 & 5.215 & 0.806 & 0.720 & 14.464 & 0.491 & 51.175 & 2.697 & 0.745 & 100.000 \\
\hline MB3N200 & N200 & Measured & 4.483 & 8.959 & 0.946 & 0.014 & 11.195 & 3.725 & 1.584 & 0.764 & 13.224 & 0.505 & 53.322 & 2.683 & 0.007 & 101.410 \\
\hline MB3N200 & N200 & Measured bc & 4.482 & 8.826 & 0.905 & 0.012 & 11.095 & 3.704 & 1.525 & 0.761 & 12.878 & 0.516 & 53.569 & 2.771 & 0.005 & 101.049 \\
\hline MB3N200 & N200 & Targeted & 4.296 & 8.940 & 0.954 & 0.000 & 10.987 & 3.725 & 1.551 & 0.720 & 12.974 & 0.491 & 52.665 & 2.697 & 0.000 & 100.000 \\
\hline MB3N303 & N303 & Measured & 6.174 & 14.916 & 0.961 & 0.011 & 11.963 & 7.562 & 0.042 & 0.753 & 5.004 & 0.490 & 51.932 & 2.821 & 0.001 & 102.630 \\
\hline MB3N303 & N303 & Measured bc & 6.165 & 14.548 & 0.909 & 0.011 & 11.399 & 7.486 & 0.041 & 0.773 & 4.874 & 0.513 & 51.223 & 2.881 & 0.001 & 100.824 \\
\hline MB3N303 & N303 & Targeted & 6.002 & 14.997 & 0.954 & 0.000 & 10.987 & 7.539 & 0.061 & 0.720 & 4.779 & 0.491 & 50.772 & 2.697 & 0.000 & 99.999 \\
\hline MB3N304 & N304 & Measured & 6.155 & 5.071 & 0.968 & 0.014 & 11.102 & 4.376 & 0.032 & 0.748 & 18.265 & 0.493 & 51.236 & 2.700 & 0.001 & 101.161 \\
\hline MB3N304 & N304 & Measured bc & 6.255 & 5.019 & 0.916 & 0.013 & 11.173 & 4.405 & 0.031 & 0.760 & 18.074 & 0.515 & 52.196 & 2.748 & 0.001 & 102.106 \\
\hline MB3N304 & N304 & Targeted & 6.002 & 4.999 & 0.954 & 0.000 & 10.987 & 4.321 & 0.061 & 0.720 & 18.241 & 0.491 & 50.526 & 2.697 & 0.000 & 99.999 \\
\hline MB3N307 & N307 & Measured & 4.540 & 9.169 & 0.972 & 0.011 & 11.488 & 8.122 & 0.031 & 0.748 & 8.509 & 0.500 & 56.478 & 2.718 & 0.004 & 103.288 \\
\hline MB3N307 & N307 & Measured bc & 4.613 & 9.073 & 0.923 & 0.011 & 11.228 & 8.177 & 0.030 & 0.766 & 8.458 & 0.525 & 55.948 & 2.765 & 0.003 & 102.522 \\
\hline MB3N307 & N307 & Targeted & 4.296 & 9.000 & 0.954 & 0.000 & 10.987 & 8.001 & 0.061 & 0.720 & 8.258 & 0.491 & 54.535 & 2.697 & 0.000 & 100.000 \\
\hline MB3N313 & $\mathrm{N} 313$ & Measured & 4.374 & 4.822 & 0.942 & 0.024 & 11.270 & 7.950 & 0.044 & 0.777 & 11.566 & 0.511 & 55.889 & 2.518 & 0.007 & 100.693 \\
\hline MB3N313 & $\mathrm{N} 313$ & Measured bc & 4.373 & 4.750 & 0.901 & 0.022 & 11.170 & 7.906 & 0.042 & 0.774 & 11.264 & 0.523 & 56.148 & 2.599 & 0.005 & 100.477 \\
\hline MB3N313 & N313 & Targeted & 4.296 & 4.999 & 0.954 & 0.000 & 10.987 & 8.001 & 0.061 & 0.720 & 11.298 & 0.491 & 55.496 & 2.697 & 0.000 & 100.000 \\
\hline MB3N314 & N314 & Measured & 4.431 & 14.852 & 0.956 & 0.107 & 11.813 & 4.327 & 0.033 & 0.735 & 9.372 & 0.498 & 49.311 & 2.409 & 1.335 & 100.179 \\
\hline MB3N314 & N314 & Measured bc & 4.453 & 14.198 & 0.908 & 0.108 & 11.543 & 4.353 & 0.033 & 0.753 & 9.314 & 0.523 & 48.841 & 2.491 & 1.049 & 98.566 \\
\hline MB3N314 & N314 & Targeted & 4.296 & 14.997 & 0.954 & 0.000 & 10.987 & 4.425 & 0.061 & 0.720 & 9.338 & 0.491 & 49.536 & 2.697 & 1.497 & 99.999 \\
\hline MB3N315 & N315 & Measured & 4.473 & 14.997 & 0.958 & 0.012 & 11.909 & 7.428 & 0.042 & 0.752 & 4.900 & 0.488 & 54.231 & 2.774 & 0.001 & 102.967 \\
\hline MB3N315 & N315 & Measured bc & 4.496 & 14.336 & 0.907 & 0.011 & 11.348 & 7.472 & 0.041 & 0.772 & 4.773 & 0.512 & 53.490 & 2.870 & 0.001 & 101.028 \\
\hline MB3N315 & N315 & Targeted & 4.296 & 14.997 & 0.954 & 0.000 & 10.987 & 7.472 & 0.061 & 0.720 & 4.779 & 0.491 & 52.545 & 2.697 & 0.000 & 99.999 \\
\hline MB3N320 & N320 & Measured & 4.426 & 5.917 & 0.954 & 0.014 & 11.416 & 5.845 & 0.044 & 0.778 & 14.221 & 0.507 & 55.568 & 2.668 & 0.007 & 102.366 \\
\hline MB3N320 & N320 & Measured bc & 4.448 & 5.656 & 0.912 & 0.012 & 11.315 & 5.880 & 0.042 & 0.775 & 13.849 & 0.519 & 55.825 & 2.760 & 0.005 & 101.999 \\
\hline MB3N320 & N320 & Targeted & 4.296 & 5.960 & 0.954 & 0.000 & 10.987 & 5.960 & 0.061 & 0.720 & 13.719 & 0.491 & 54.155 & 2.697 & 0.000 & 100.000 \\
\hline MB3N322 & N322 & Measured & 4.355 & 5.884 & 0.961 & 0.015 & 11.677 & 3.703 & 0.032 & 0.765 & 12.452 & 0.506 & 59.152 & 2.559 & 0.092 & 102.152 \\
\hline MB3N322 & N322 & Measured bc & 4.377 & 5.624 & 0.912 & 0.015 & 11.408 & 3.725 & 0.032 & 0.783 & 12.375 & 0.531 & 58.594 & 2.647 & 0.072 & 101.096 \\
\hline MB3N322 & N322 & Targeted & 4.296 & 5.960 & 0.954 & 0.000 & 10.987 & 3.725 & 0.061 & 0.720 & 12.229 & 0.491 & 57.880 & 2.697 & 0.000 & 100.000 \\
\hline MB3N323 & N323 & Measured & 4.440 & 11.020 & 0.959 & 0.011 & 11.838 & 3.859 & 0.039 & 0.749 & 11.263 & 0.486 & 56.959 & 2.686 & 0.056 & 104.365 \\
\hline
\end{tabular}




\begin{tabular}{|c|c|c|c|c|c|c|c|c|c|c|c|c|c|c|c|c|}
\hline $\begin{array}{c}\text { Glass } \\
\text { ID }\end{array}$ & $\begin{array}{c}\begin{array}{c}\text { Glass ID } \\
\text { (shortened) }\end{array} \\
\end{array}$ & $\begin{array}{c}\text { Compositional } \\
\text { View }\end{array}$ & $\mathbf{A l}_{2} \mathbf{O}_{3}$ & $\mathbf{B}_{2} \mathbf{O}_{3}$ & $\mathrm{CaO}$ & $\mathrm{Cr}_{2} \mathrm{O}_{3}$ & $\mathrm{Fe}_{2} \mathrm{O}_{3}$ & $\mathbf{L i}_{2} \mathbf{O}$ & MgO & $\mathrm{MnO}$ & $\mathrm{Na}_{2} \mathrm{O}$ & $\mathrm{NiO}$ & $\mathrm{SiO}_{2}$ & $\mathbf{U}_{3} \mathbf{O}_{8}$ & $\mathrm{ZrO}_{2}$ & $\begin{array}{l}\text { Sum of } \\
\text { Oxides }\end{array}$ \\
\hline MB3N323 & N323 & Measured bc & 4.439 & 10.855 & 0.908 & 0.011 & 11.280 & 3.838 & 0.038 & 0.769 & 10.971 & 0.509 & 56.183 & 2.774 & 0.045 & 102.620 \\
\hline MB3N323 & $\mathrm{N} 323$ & Targeted & 4.296 & 11.175 & 0.954 & 0.000 & 10.987 & 3.867 & 0.061 & 0.720 & 10.947 & 0.491 & 53.804 & 2.697 & 0.000 & 99.999 \\
\hline MB3N324 & $\mathrm{N} 324$ & Measured & 4.398 & 11.358 & 0.972 & 0.018 & 11.023 & 6.125 & 0.028 & 0.741 & 8.792 & 0.470 & 54.873 & 2.694 & 0.001 & 101.495 \\
\hline MB3N324 & N324 & Measured bc & 4.420 & 10.858 & 0.920 & 0.017 & 11.097 & 6.162 & 0.028 & 0.753 & 8.700 & 0.491 & 55.897 & 2.788 & 0.001 & 102.131 \\
\hline MB3N324 & N324 & Targeted & 4.296 & 11.175 & 0.954 & 0.000 & 10.987 & 6.169 & 0.061 & 0.720 & 8.645 & 0.491 & 53.804 & 2.697 & 0.000 & 99.999 \\
\hline MB3N326 & $\mathrm{N} 326$ & Measured & 4.521 & 5.989 & 0.978 & 0.022 & 11.055 & 6.082 & 0.816 & 0.750 & 13.369 & 0.487 & 54.820 & 2.756 & 0.001 & 101.646 \\
\hline MB3N326 & $\mathrm{N} 326$ & Measured bc & 4.514 & 5.843 & 0.925 & 0.021 & 11.126 & 6.022 & 0.802 & 0.763 & 13.225 & 0.509 & 55.842 & 2.815 & 0.001 & 102.406 \\
\hline MB3N326 & N326 & Targeted & 4.296 & 5.960 & 0.954 & 0.000 & 10.987 & 5.960 & 0.806 & 0.720 & 12.974 & 0.491 & 54.155 & 2.697 & 0.000 & 100.000 \\
\hline MB3U165 & U165 & Measured & 4.204 & 7.285 & 0.972 & 0.019 & 10.769 & 5.210 & 0.851 & 0.718 & 15.232 & 0.461 & 51.985 & 2.273 & 0.686 & 100.665 \\
\hline MB3U165 & U165 & Measured bc & 4.203 & 7.177 & 0.920 & 0.018 & 10.841 & 5.182 & 0.836 & 0.729 & 15.071 & 0.482 & 52.961 & 2.348 & 0.546 & 101.313 \\
\hline MB3U165 & U165 & Targeted & 4.192 & 7.450 & 0.941 & 0.000 & 10.824 & 5.215 & 0.806 & 0.691 & 14.944 & 0.460 & 51.168 & 2.563 & 0.745 & 99.999 \\
\hline MB3U200 & U200 & Measured & 4.384 & 8.927 & 0.945 & 0.016 & 11.459 & 3.741 & 1.572 & 0.724 & 13.692 & 0.497 & 53.215 & 2.671 & 0.001 & 101.845 \\
\hline MB3U200 & U200 & Measured bc & 4.455 & 8.837 & 0.897 & 0.016 & 11.199 & 3.766 & 1.557 & 0.742 & 13.609 & 0.523 & 52.717 & 2.718 & 0.001 & 101.037 \\
\hline MB3U200 & U200 & & 4.192 & & 0.941 & & 10.824 & 3.725 & 1.551 & 0.691 & 13.454 & 0.460 & 52.658 & 2.563 & 0.000 & 99.999 \\
\hline MB3U303 & U303 & Measured & 6.231 & 14.836 & 0.982 & 0.020 & 11.466 & 7.718 & 0.044 & 0.738 & 5.416 & 0.494 & 52.199 & 2.683 & 0.008 & 102.833 \\
\hline MB3U303 & U303 & Measured bc & 6.221 & 14.470 & 0.932 & 0.020 & 11.205 & 7.640 & 0.043 & 0.756 & 5.382 & 0.520 & 51.703 & 2.740 & 0.006 & 101.639 \\
\hline MB3U303 & U303 & Targeted & 5.898 & 14.997 & 0.941 & 0.000 & 10.824 & 7.539 & 0.061 & 0.691 & 5.259 & 0.460 & 50.766 & 2.563 & 0.000 & 99.999 \\
\hline MB3U304 & U304 & Measured & 5.961 & 4.910 & 0.956 & 0.072 & 11.327 & 4.284 & 0.036 & 0.724 & 19.310 & 0.497 & 51.611 & 2.529 & 0.004 & 102.223 \\
\hline MB3U304 & U304 & Measured bc & 6.058 & 4.859 & 0.908 & 0.072 & 11.066 & 4.313 & 0.036 & 0.742 & 19.192 & 0.523 & 51.122 & 2.573 & 0.003 & 101.467 \\
\hline MB3U304 & U304 & Targeted & 5.898 & 4.999 & 0.941 & 0.000 & 10.824 & 4.321 & 0.061 & 0.691 & 18.721 & 0.460 & 50.520 & 2.563 & 0.000 & 99.999 \\
\hline MB3U307 & U307 & Measured & 4.450 & 8.823 & 0.921 & 0.014 & 11.252 & 7.993 & 0.046 & 0.747 & 9.028 & 0.461 & 55.461 & 2.597 & 0.058 & 101.851 \\
\hline MB3U307 & U307 & Measured bc & 4.449 & 8.692 & 0.881 & 0.012 & 11.153 & 7.950 & 0.043 & 0.744 & 8.792 & 0.472 & 55.719 & 2.682 & 0.041 & 101.630 \\
\hline MB3U307 & U307 & Targeted & 4.192 & 9.000 & 0.941 & 0.000 & 10.824 & 8.001 & 0.061 & 0.691 & 8.738 & 0.460 & 54.528 & 2.563 & 0.000 & 99.999 \\
\hline MB3U313 & U313 & Measured & 4.318 & 4.999 & 0.948 & 0.012 & 11.152 & 7.890 & 0.035 & 0.704 & 11.761 & 0.489 & 56.050 & 2.509 & 0.001 & 100.868 \\
\hline MB3U313 & U313 & Measured bc & 4.311 & 4.875 & 0.897 & 0.011 & 11.235 & 7.810 & 0.035 & 0.716 & 11.638 & 0.511 & 57.100 & 2.562 & 0.001 & 101.703 \\
\hline MB3U313 & U313 & Targeted & 4.192 & 4.999 & 0.941 & 0.000 & 10.824 & 8.001 & 0.061 & 0.691 & 11.778 & 0.460 & 55.489 & 2.563 & 0.000 & 99.999 \\
\hline MB3U314 & U314 & Measured & 4.110 & 13.000 & 0.905 & 0.021 & 10.480 & 3.983 & 0.073 & 0.705 & 9.554 & 0.440 & 53.269 & 2.488 & 1.375 & 100.402 \\
\hline MB3U314 & U314 & Measured bc & 4.176 & 12.870 & 0.866 & 0.019 & 10.387 & 4.010 & 0.070 & 0.702 & 9.304 & 0.450 & 53.516 & 2.532 & 0.972 & 99.874 \\
\hline MB3U314 & U314 & Targeted & 4.192 & 14.997 & 0.941 & 0.000 & 10.824 & 4.425 & 0.061 & 0.691 & 9.819 & 0.460 & 49.529 & 2.563 & 1.497 & 99.999 \\
\hline MB3U315 & U315 & Measured & 4.445 & 14.506 & 0.932 & 0.012 & 10.694 & 7.546 & 0.036 & 0.698 & 6.379 & 0.458 & 52.092 & 2.624 & 0.001 & 100.423 \\
\hline MB3U315 & U315 & Measured bc & 4.517 & 14.355 & 0.881 & 0.011 & 10.762 & 7.597 & 0.035 & 0.709 & 6.312 & 0.479 & 53.040 & 2.669 & 0.001 & 101.370 \\
\hline MB3U315 & U315 & Targeted & 4.192 & 14.997 & 0.941 & 0.000 & 10.824 & 7.472 & 0.061 & 0.691 & 5.259 & 0.460 & 52.539 & 2.563 & 0.000 & 99.999 \\
\hline MB3U320 & U320 & Measured & 4.379 & 5.949 & 0.949 & 0.012 & 11.259 & 6.017 & 0.032 & 0.718 & 14.424 & 0.451 & 55.622 & 2.553 & 0.001 & 102.364 \\
\hline
\end{tabular}




\begin{tabular}{|c|c|c|c|c|c|c|c|c|c|c|c|c|c|c|c|c|}
\hline $\begin{array}{c}\text { Glass } \\
\text { ID }\end{array}$ & $\begin{array}{c}\begin{array}{c}\text { Glass ID } \\
\text { (shortened) }\end{array} \\
\end{array}$ & $\begin{array}{c}\text { Compositional } \\
\text { View } \\
\end{array}$ & $\mathbf{A l}_{2} \mathbf{O}_{3}$ & $\mathbf{B}_{2} \mathbf{O}_{3}$ & $\mathrm{CaO}$ & $\mathrm{Cr}_{2} \mathrm{O}_{3}$ & $\mathrm{Fe}_{2} \mathrm{O}_{3}$ & $\mathbf{L i}_{2} \mathbf{O}$ & MgO & MnO & $\mathrm{Na}_{2} \mathrm{O}$ & $\mathrm{NiO}$ & $\mathrm{SiO}_{2}$ & $\mathbf{U}_{3} \mathbf{O}_{8}$ & $\mathrm{ZrO}_{2}$ & $\begin{array}{l}\text { Sum of } \\
\text { Oxides } \\
\end{array}$ \\
\hline "MB3U320 & U320 & Measured bc & 4.372 & 5.802 & 0.901 & 0.012 & "11.001 & 5.957 & 0.031 & 0.735 & 14.335 & 0.474 & 55.097 & 2.607 & 0.001 & 101.325 \\
\hline MB3U320 & U320 & Targeted & 4.192 & 5.960 & 0.941 & 0.000 & 10.824 & 5.960 & 0.061 & 0.691 & 14.199 & 0.460 & 54.148 & 2.563 & 0.000 & 99.999 \\
\hline MB3U322 & U322 & Measured & 4.237 & 5.892 & 0.927 & 0.029 & 11.434 & 3.644 & 0.050 & 0.725 & 12.739 & 0.450 & 60.489 & 2.450 & 0.005 & 103.071 \\
\hline MB3U322 & U322 & Measured bc & 4.258 & 5.633 & 0.877 & 0.028 & 10.895 & 3.666 & 0.049 & 0.745 & 12.408 & 0.471 & 59.663 & 2.534 & 0.004 & 101.232 \\
\hline MB3U322 & U322 & Targeted & 4.192 & 5.960 & 0.941 & 0.000 & 10.824 & 3.725 & 0.061 & 0.691 & 12.709 & 0.460 & 57.873 & 2.563 & 0.000 & 99.999 \\
\hline MB3U323 & U323 & Measured & 4.454 & 11.068 & 0.918 & 0.015 & 10.952 & 3.864 & 0.046 & 0.734 & 11.606 & 0.503 & 54.927 & 2.565 & 0.136 & 101.788 \\
\hline MB3U323 & U323 & Measured bc & 4.448 & 10.797 & 0.878 & 0.013 & 10.854 & 3.826 & 0.043 & 0.732 & 11.303 & 0.515 & 55.181 & 2.619 & 0.096 & 101.303 \\
\hline MB3U323 & U323 & Targeted & 4.192 & 11.175 & 0.941 & 0.000 & 10.824 & 3.867 & 0.061 & 0.691 & 11.428 & 0.460 & 53.798 & 2.563 & 0.000 & 100.000 \\
\hline MB3U324 & U324 & Measured & 4.398 & 10.859 & 0.961 & 0.027 & 11.763 & 6.141 & 0.051 & 0.730 & 9.460 & 0.469 & 55.247 & 2.597 & 0.001 & 102.704 \\
\hline MB3U324 & U324 & Measured bc & 4.391 & 10.590 & 0.910 & 0.026 & 11.208 & 6.079 & 0.050 & 0.750 & 9.214 & 0.491 & 54.492 & 2.653 & 0.001 & 100.854 \\
\hline MB3U324 & U324 & Targeted & 4.192 & 11.175 & 0.941 & 0.000 & 10.824 & 6.169 & 0.061 & 0.691 & 9.126 & 0.460 & 53.798 & 2.563 & 0.000 & 100.000 \\
\hline MB3U326 & U326 & Measured & 4.322 & 6.070 & 0.942 & 0.015 & 11.727 & 5.867 & 0.829 & 0.731 & 13.918 & 0.494 & 54.927 & 2.547 & 0.001 & 102.390 \\
\hline MB3U326 & U326 & Measured bc & 4.344 & 5.802 & 0.892 & 0.015 & 11.174 & 5.902 & 0.811 & 0.751 & 13.557 & 0.517 & 54.177 & 2.635 & 0.001 & 100.577 \\
\hline MB3U326 & U326 & Targeted & 4.192 & 5.960 & 0.941 & 0.000 & 10.824 & 5.960 & 0.806 & 0.691 & 13.454 & 0.460 & 54.148 & 2.563 & 0.000 & 99.999 \\
\hline
\end{tabular}


Exhibit E7 in Appendix E provides plots that compare measured and measure bias-corrected values to targeted values by oxide for the study glasses. A plot of the sum of oxides is also provided in this exhibit. As seen in Table 14 and Exhibit E7, no major problems are seen in the agreement among the measured, measured bias-corrected, and targeted compositional views of the MB3 glasses. All three of these compositional views will be utilized to represent the MB3 glasses in the discussions that follow to ensure that there are no differences in the conclusions.

\subsection{DURABILITY}

Samples of the MB3 study glasses, after being batched and fabricated (via quenching), were subjected to a second heat treatment - they were cooled to simulate a centerline canister cooling profile. Differences in glass durability for these two cooling regimes (quenched versus centerline cooled) are of interest to this study. The investigation into this question required durability to be measured for the quenched and centerline cooled versions of each of the study glasses.

The 7-day Product Consistency Test (PCT) was used as the assessment of glass durability. More specifically, Method A of the PCT (ASTM C1285-97) was used for these measurements. The PCTs were conducted in triplicate for the study glasses in two sets: set \#1 included the glasses representing nominally washed sludge, and set \#2 included glasses representing the underwashed sludge. In addition, PCTs were also conducted in triplicate for samples of the EA glass, the ARM glass, and a blank (ASTM Type I water) for each set. Analytical plans supporting these tests were provided in the form of memoranda (see Appendices C and D). The plans assisted the SRTC-ML in measuring the compositions of the solutions resulting from the two groups of PCTs. Of primary interest were the concentrations (in parts per million, ppm) of boron (B), lithium (Li), sodium (Na), and silicon ( $\mathrm{Si}$ ). Samples of a multi-element solution standard were also included in each analytical plan (as a check on the accuracy of the ICP - AES used for these measurements).

The results from these tests are given in Table E3 (for set \#1) and Table E4 (for set \#2) of Appendix E. The PCT results for the centerline-cooled version of each study glass are indicated by the "clc" suffix on the glass ID. One of the quality control checkpoints for the PCT procedure is solution weight loss over the course of the 7-day test. The shaded entries of Tables E3 and E4 indicate those solutions that fell outside the weight-loss guidelines (weight loss must be less than $5 \mathrm{wt} \%$ ). At least two successful solutions out of the 3 conducted for a glass are required to generate a representative PCT for that glass. Although this criterion has not been met for all of 
the MB3 study glasses, the results are believed to provide meaningful and representative comparisons for assessing the impact of the cooling regimes since the impact must be larger than the measurement variability to be of consequence.

Any measurement in the "as reported" columns of Tables E3 and E4 proceeded by a "<" was below the detection limit for the ICP, and the measurement was replaced by $1 / 2$ of the detection limit in the determination of the parts per million ( $\mathrm{ppm}$ ) columns of the tables. The values in the ppm columns were also adjusted for the dilution factors by multiplying the "as-reported" values by 1.6667 for the MB3 and ARM glasses and by 16.6667 for the EA glass. Thus, the concentrations in the ppm columns reflect detection and dilution adjustments.

\subsubsection{PCT RESULTS FOR THE GLASSES REPRESENTING THE NOMINALLY WASHED SLUDGE}

Exhibit E8 in Appendix E provides plots of the leachate concentrations and standards in the analytical sequence reported by the SRTC-ML for the PCTs from the nominally washed case. These plots include the values from the EA PCTs and the blanks. These values expand the scales of these plots, making it difficult to distinguish among the results of the other analyses. Exhibit E9 in Appendix E provides these same plots excluding the EA and blank, yielding a clearer picture of the behavior of the PCTs for the other glasses and standards.

Exhibit E10 in Appendix E provides plots of the leachate concentrations for each type of submitted solution: the standards, the blanks, EA, ARM, and the study glasses representing the nominally washed case. Once again, excluding the results for EA and the blanks improves the opportunity for investigating the behavior of the PCTs for the other glasses and standards. Such results are shown in Exhibit E11 in Appendix E.

PCT leachate concentrations are typically normalized using the corresponding cation concentration (expressed as a weight percent) in the glass to obtain a grams-per-liter (g/L) leachate concentration. The normalization of the PCTs is usually conducted using the measured compositions of the glasses. This is the preferred normalization process for the PCTs. For completeness, the targeted cation and the bias-corrected cation concentrations will also be used to conduct this normalization. 
As is the usual convention, the common logarithm of the normalized PCT (normalized leachate,

NL) for each element of interest will be determined and used for comparison. To accomplish this computation, one must

1. Determine the common logarithm of the elemental parts per million (ppm) leachate concentration for each of the triplicates and each of the elements of interest (Table E3 of Appendix E provides these values),

2. Average the common logarithms over the triplicates for each element of interest, and then

Normalizing Using Measured Composition (preferred method)

3. Subtract a quantity equal to 1 plus the common logarithm of the average cation measured concentration (expressed as a weight percent of the glass) from the average computed in step 2 .

\section{Or Normalizing Using Target Composition}

3. Subtract a quantity equal to 1 plus the common logarithm of the target cation concentration (expressed as a weight percent of the glass) from the average computed in step 2 .

\section{Or Normalizing Using Measured Bias-Corrected Composition}

3. Subtract a quantity equal to 1 plus the common logarithm of the measured biascorrected cation concentration (expressed as a weight percent of the glass) from the average computed in step 2 .

As a preliminary step to completing these normalizations of the PCTs, statistical analyses were conducted of the results from the three analyses of the multi-element standard solution per analytical block. Exhibit E12 in Appendix E provides these analyses. Although there appears to be statistical differences among the block averages for most of the elements of interest, no bias correction of the PCT results for the study glasses was conducted. This approach was taken since the triplicate PCTs for a single study glass were placed in different ICP blocks. Averaging the ppm results for each set of triplicates helps to minimize the impact of the ICP effects.

Table 15 presents the block averages of the solution standards, and they indicate consistent and reasonably accurate results (e.g., most differences of overall average versus reference value < $5 \%$ ) from these analyses. 


\section{Table 15: Average Measurements of Standard Solution by Analytical Block - PCTs for Glasses from Nominally Washed Sludge.}

\begin{tabular}{|c|c|c|c|c||}
\hline \multirow{2}{*}{$\begin{array}{c}\text { Analytical } \\
\text { Block }\end{array}$} & $\mathbf{4}$ Avg \\
\cline { 2 - 5 } & $\mathbf{B p m})$ & $\begin{array}{c}\mathbf{L i} \\
(\mathbf{p p m})\end{array}$ & $\begin{array}{c}\mathbf{N a} \\
(\mathbf{p p m})\end{array}$ & $\mathbf{S i}(\mathbf{p p m})$ \\
\hline \hline 1 & 20.8 & 10.7 & 74.0 & 51.7 \\
\hline 2 & 18.6 & 10.5 & 77.4 & 46.9 \\
\hline 3 & 19.2 & 10.7 & 77.5 & 46.4 \\
\hline 4 & 18.8 & 10.7 & 78.5 & 48.2 \\
\hline 5 & 18.9 & 10.7 & 78.3 & 47.4 \\
\hline 6 & 18.9 & 11.0 & 80.4 & 49.3 \\
\hline Grand Average & 19.2 & 10.7 & 77.7 & 48.3 \\
\hline Reference Value & 20 & 10 & 81 & 50 \\
\hline \% difference & $-4.0 \%$ & $7.0 \%$ & $-4.1 \%$ & $-3.3 \%$ \\
\hline \hline
\end{tabular}

Table 16 provides the results from the normalization process using the information in Table 15 and all of the data of Table E3 (i.e., before screening the PCT results for solution-weight problems). Exhibit E13 in Appendix E provides scatter plots for these results (both quenched and centerline cooled) offering an opportunity to investigate the consistency in the leaching across the elements for the glasses of this study. The consistency is typically demonstrated by a high degree of linear correlation among the values. PCT values normalized using targeted, measured, and bias-corrected compositions were investigated. A high degree of correlation is seen for these data for some pairs of elements. However, small correlations (as small as 58\%) can be seen between the data for some pairs of elements (e.g., particularly between $\mathrm{B}$ and $\mathrm{Na}$ ). While poor correlation coefficients are expected with $\mathrm{Si}$ in the PCT (due to Si saturation in solution), $\mathrm{Na}, \mathrm{B}$, and $\mathrm{Li}$ should be correlated to a relatively high degree. Low correlations found in this study may indicate that some of the glasses are phase separated (e.g., amorphous phase separation). Similar trends were observed by Jantzen et al. (1999). 
Table 16. Normalized PCTs before Screening for Solution-Weight Problems - Nominally Washed Sludge.

\begin{tabular}{|c|c|c|c|c|c|c|c|c|c|c|c|c|c|c|c|c|c|}
\hline \multirow[b]{2}{*}{$\begin{array}{l}\text { Glass } \\
\text { ID }\end{array}$} & \multirow[b]{2}{*}{ Composition } & \multicolumn{8}{|c|}{ Quenched } & \multicolumn{8}{|c|}{ Centerline Cooled } \\
\hline & & $\left.\begin{array}{c}\log \mathbf{N L} \\
{[\mathbf{B}(\mathrm{g} / \mathbf{L})]}\end{array}\right]$ & $\left.\begin{array}{c}\log N \mathbf{N L} \\
{[\mathbf{L i}(\mathrm{g} / \mathrm{L})]}\end{array}\right]$ & $\begin{array}{c}\log \mathbf{N L} \\
{[\mathrm{Na}(\mathrm{g} / \mathrm{L})]}\end{array}$ & $\left.\begin{array}{c}\log \mathbf{N L} \\
{[\mathrm{Si}(\mathrm{g} / \mathrm{L})]}\end{array}\right]$ & $\begin{array}{c}\mathbf{N L} \\
\mathbf{B}(\mathbf{g} / \mathbf{L})\end{array} \mid$ & $\begin{array}{c}\mathbf{N L} \\
\mathbf{L i}(\mathbf{g} / \mathbf{L})\end{array}$ & $\begin{array}{c}\mathrm{NL} \\
\mathrm{Na}(\mathrm{g} / \mathrm{L})\end{array}$ & $\mid \begin{array}{c}\mathbf{N L} \\
\mathrm{Si}(\mathrm{g} / \mathrm{L})\end{array}$ & \begin{tabular}{|l|}
$\log \mathbf{~ N L}$ \\
{$[\mathbf{B}(\mathrm{g} / \mathbf{L})]$}
\end{tabular} & $\begin{array}{c}\log \mathbf{N L} \\
{[\mathrm{Li}(\mathrm{g} / \mathrm{L})]}\end{array}$ & {$\left[\begin{array}{c}\log \mathbf{N L} \\
{[\mathrm{Na}(\mathrm{g} / \mathrm{L})]}\end{array}\right]$} & $\left|\begin{array}{c}\log \mathbf{N L} \\
{[\mathrm{Si}(\mathrm{g} / \mathrm{L})]}\end{array}\right|$ & $\begin{array}{c}\mathbf{N L} \\
\mathbf{B}(\mathrm{g} / \mathrm{L})\end{array}$ & $\left|\begin{array}{c}\mathbf{N L} \\
\mathbf{L i}(\mathbf{g} / \mathbf{L})\end{array}\right|$ & $\begin{array}{c}\mathrm{NL} \\
\mathrm{Na}(\mathrm{g} / \mathrm{L})\end{array}$ & $\begin{array}{c}\mathbf{N L} \\
\mathrm{Si}(\mathrm{g} / \mathrm{L})\end{array}$ \\
\hline ARM & see $[8]$ & -0.2861 & -0.1716 & -0.2812 & \begin{tabular}{|r|}
-0.5482 \\
\end{tabular} & 0.52 & 0.67 & 0.52 & 0.28 & & & & & & & & \\
\hline EA & see $[8]$ & 1.1976 & 0.9791 & 1.0689 & 0.5506 & 15.76 & 9.53 & 11.72 & 3.55 & & & & & & & & \\
\hline N165 & Measured & -0.0046 & 0.0439 & 0.0493 & -0.2108 & 0.99 & 1.11 & 1.12 & 0.62 & $\begin{array}{l}-0.0348 \\
\end{array}$ & 0.0309 & $\begin{array}{l}-0.0044 \\
\end{array}$ & -0.2222 & 0.92 & 1.07 & 0.99 & 0.60 \\
\hline N165 & Measured bc & 0.0019 & 0.0463 & 0.0539 & -0.2188 & 1.00 & 1.11 & 1.13 & 0.60 & -0.0283 & 0.0333 & 0.0002 & -0.2302 & 0.94 & 1.08 & 1.00 & 0.59 \\
\hline N165 & Targeted & -0.0143 & 0.0372 & 0.0601 & -0.2000 & 0.97 & 1.09 & 1.15 & 0.63 & -0.0445 & 0.0242 & 0.0064 & -0.2114 & 0.90 & 1.06 & 1.01 & 0.61 \\
\hline N200 & Measured & -0.1145 & -0.0451 & -0.1169 & -0.3155 & 0.77 & 0.90 & 0.76 & 0.48 & -0.1352 & -0.0665 & -0.1453 & -0.3215 & 0.73 & 0.86 & 0.72 & 0.48 \\
\hline N200 & Measured bc & -0.1079 & -0.0427 & -0.1054 & -0.3175 & 0.78 & 0.91 & 0.78 & 0.48 & -0.1287 & -0.0641 & -0.1338 & -0.3235 & 0.74 & 0.86 & 0.73 & 0.47 \\
\hline $\mathrm{N} 200$ & Targeted & -0.1135 & -0.0451 & -0.1086 & -0.3101 & 0.77 & 0.90 & 0.78 & 0.49 & -0.1343 & -0.0665 & -0.1370 & -0.3161 & 0.73 & 0.86 & 0.73 & 0.48 \\
\hline N303 & Measured & -0.1023 & -0.0443 & -0.4328 & -0.3010 & 0.79 & 0.90 & 0.37 & 0.50 & -0.1348 & -0.0808 & -0.3797 & -0.3161 & 0.73 & 0.83 & 0.42 & 0.48 \\
\hline $\mathrm{N} 303$ & Measured bc & -0.0914 & -0.0399 & -0.4214 & -0.2950 & 0.81 & 0.91 & 0.38 & 0.51 & -0.1239 & -0.0764 & -0.3683 & -0.3101 & 0.75 & 0.84 & 0.43 & 0.49 \\
\hline $\mathrm{N} 303$ & Targeted & -0.1046 & -0.0429 & -0.4128 & -0.2912 & 0.79 & 0.91 & 0.39 & 0.51 & $\begin{array}{l}-0.1371 \\
\end{array}$ & -0.0794 & $\begin{array}{l}-0.3597 \\
\end{array}$ & $\begin{array}{l}-0.3063 \\
\end{array}$ & 0.73 & 0.83 & 0.44 & 0.49 \\
\hline N304 & Measured & 0.0292 & 0.0956 & 0.2955 & -0.0638 & 1.07 & 1.25 & 1.97 & 0.86 & 0.0191 & 0.1030 & 0.2700 & -0.0557 & 1.05 & 1.27 & 1.86 & 0.88 \\
\hline N304 & Measured bc & 0.0337 & 0.0926 & 0.2991 & -0.0719 & 1.08 & 1.24 & 1.99 & 0.85 & 0.0236 & 0.1001 & 0.2736 & -0.0637 & 1.06 & 1.26 & 1.88 & 0.86 \\
\hline N304 & Targeted & 0.0354 & 0.1010 & 0.2186 & .0577 & 1.09 & 1.26 & 1.65 & 0.88 & 0.0253 & 0.1085 & 0.1931 & -0.0496 & 1.06 & 1.28 & 1.56 & 0.89 \\
\hline N307 & Measured & -0.0518 & 0.0385 & -0.1338 & $\begin{array}{l}-0.1956 \\
\end{array}$ & 0.89 & 1.09 & 0.73 & 0.64 & $\begin{array}{l}-0.0277 \\
\end{array}$ & 0.1534 & -0.0751 & -0.0960 & 0.94 & 1.42 & 0.84 & 0.80 \\
\hline N307 & Measured bc & $\begin{array}{l}-0.0473 \\
\end{array}$ & 0.0355 & -0.1312 & $\begin{array}{l}-0.1915 \\
\end{array}$ & 0.90 & 1.09 & 0.74 & 0.64 & -0.0231 & 0.1505 & -0.0725 & -0.0919 & 0.95 & 1.41 & 0.85 & 0.81 \\
\hline N307 & Targeted & -0.0437 & 0.0450 & -0.1208 & -0.1804 & 0.90 & 1.11 & 0.76 & 0.66 & -0.0196 & 0.1600 & -0.0621 & -0.0808 & 0.96 & 1.45 & 0.87 & 0.83 \\
\hline N313 & Measured & 0.1154 & 0.1773 & 0.1070 & -0.0028 & 1.30 & 1.50 & 1.28 & 0.99 & 0.2499 & 0.4336 & 0.2001 & 0.2005 & 1.78 & 2.71 & 1.59 & 1.59 \\
\hline N313 & Measured bc & 0.1219 & 1797 & 0.1185 & -0.0048 & 1.32 & 1.51 & 1.31 & 0.99 & 0.2564 & 0.4360 & 0.2116 & .1985 & 1.80 & 2.73 & 1.63 & 1.58 \\
\hline N313 & Targeted & 0.0997 & 1745 & 0.1172 & 0.0003 & 1.26 & 1.49 & 1.31 & 1.00 & 0.2342 & 0.4308 & 0.2103 & 0.2036 & 1.71 & 2.70 & 1.62 & 1.60 \\
\hline N314 & asured & .0071 & 0928 & -0.1825 & $\begin{array}{l}-0.3997 \\
\end{array}$ & 1.02 & 1.24 & 0.66 & 0.40 & -0.0512 & 0.0447 & -0.2050 & -0.3969 & 0.89 & 1.11 & 0.62 & 0.40 \\
\hline N314 & Measured bc & 0.0267 & 0.0902 & -0.1798 & -0.3955 & 1.06 & 1.23 & 0.66 & 0.40 & -0.0316 & 0.0421 & -0.2023 & -0.3928 & 0.93 & 1.10 & 0.63 & 0.40 \\
\hline N314 & Targeted & 0.0029 & 0831 & -0.1810 & -0.4017 & 1.01 & 1.21 & 0.66 & 0.40 & -0.0554 & 0.0349 & -0.2034 & -0.3989 & 0.88 & 1.08 & 0.63 & 0.40 \\
\hline N315 & Measured & 0.0902 & 1353 & -0.1579 & -0.2389 & 1.23 & 1.37 & 0.70 & 0.58 & 0.0898 & 0.1296 & -0.1186 & -0.2423 & 1.23 & 1.35 & 0.76 & 0.57 \\
\hline N315 & Measured bc & 0.1098 & 0.1327 & -0.1464 & -0.2329 & 1.29 & 1.36 & 0.71 & 0.58 & 0.1094 & 0.1270 & -0.1072 & -0.2364 & 1.29 & 1.34 & 0.78 & 0.58 \\
\hline N315 & & 0.0902 & & -0.1470 & -0.2252 & 1.23 & 1.36 & 0.71 & 0.60 & 0.0898 & 0.1270 & -0.1078 & -0.2286 & 1.23 & 1.34 & 0.78 & 0.59 \\
\hline N320 & Measured & 0.0001 & 0.1177 & 0.0979 & $\begin{array}{l}-0.1089 \\
\end{array}$ & 1.00 & 1.31 & 1.25 & 0.78 & -0.0104 & 0.1454 & 0.0769 & -0.0947 & 0.98 & 1.40 & 1.19 & 0.80 \\
\hline N320 & Measured bc & & & 1094 & & 1.05 & 1.30 & 1.29 & 0.77 & 0.0092 & & .0884 & -0.0967 & 1.02 & 1.39 & 1.23 & 0.80 \\
\hline N320 & Targeted & .0031 & 1092 & .1135 & $\begin{array}{l}-0.0977 \\
\end{array}$ & 0.99 & 1.29 & 1.30 & 0.80 & -0.0135 & 0.1369 & 0.0925 & -0.0835 & 0.97 & 1.37 & 1.24 & 0.83 \\
\hline N322 & Measured & $\begin{array}{l}-0.2463 \\
\end{array}$ & -0.1005 & -0.2249 & -0.3362 & 0.57 & 0.79 & 0.60 & 0.46 & -0.2314 & $\begin{array}{l}-0.0984 \\
\end{array}$ & -0.2368 & -0.3206 & 0.59 & 0.80 & 0.58 & 0.48 \\
\hline N322 & Measured bc & $\begin{array}{l}-0.2267 \\
\end{array}$ & -0.1031 & -0.2222 & \begin{tabular}{l|}
-0.3321 \\
\end{tabular} & 0.59 & 0.79 & 0.60 & 0.47 & -0.2118 & -0.1010 & -0.2342 & -0.3165 & 0.61 & 0.79 & 0.58 & 0.48 \\
\hline N322 & Targeted & -0.2519 & -0.1031 & -0.2171 & -0.3268 & 0.56 & 0.79 & 0.61 & 0.47 & -0.2370 & -0.1010 & -0.2290 & -0.3112 & 0.58 & 0.79 & 0.59 & 0.49 \\
\hline N323 & & & & -0.2709 & & 0.71 & 0.88 & 0.54 & 0.42 & -0.2115 & -0.0979 & -0.2977 & -0.3969 & 0.61 & 0.80 & 0.50 & 0.40 \\
\hline N323 & Measured bc & -0.1451 & -0.0525 & -0.2595 & -0.3674 & 0.72 & 0.89 & 0.55 & 0.43 & -0.2049 & -0.0955 & -0.2863 & -0.3909 & 0.62 & 0.80 & 0.52 & 0.41 \\
\hline N323 & Targeted & -0.1578 & -0.0557 & -0.2586 & -0.3486 & 0.70 & 0.88 & 0.55 & 0.45 & -0.2175 & -0.0988 & -0.2854 & -0.3721 & 0.61 & 0.80 & 0.52 & 0.42 \\
\hline N324 & Measured & -0.0577 & 0.0155 & -0.2124 & -0.2642 & 0.88 & 1.04 & 0.61 & 0.54 & -0.0657 & 0.0189 & -0.1942 & -0.2665 & 0.86 & 1.04 & 0.64 & 0.54 \\
\hline N324 & Measured bc & -0.0382 & 0.0129 & -0.2078 & -0.2722 & 0.92 & 1.03 & 0.62 & 0.53 & -0.0461 & 0.0163 & -0.1897 & -0.2746 & 0.90 & 1.04 & 0.65 & 0.53 \\
\hline N324 & Targeted & $\begin{array}{l}-0.0507 \\
\end{array}$ & 0.0124 & -0.2051 & -0.2556 & 0.89 & 1.03 & 0.62 & 0.56 & -0.0586 & 0.0158 & -0.1869 & -0.2580 & 0.87 & 1.04 & 0.65 & 0.55 \\
\hline N326 & Measured & 0.0194 & 0.0644 & 0.0393 & -0.1359 & 1.05 & 1.16 & 1.09 & 0.73 & 0.0281 & 0.1032 & 0.0320 & -0.1208 & 1.07 & 1.27 & 1.08 & 0.76 \\
\hline N326 & Measured bc & 0.0302 & 0.0687 & 0.0441 & $\begin{array}{l}-0.1439 \\
\end{array}$ & 1.07 & 1.17 & 1.11 & 0.72 & 0.0388 & 0.1075 & $\begin{array}{l}0.0367 \\
\end{array}$ & -0.1289 & 1.09 & 1.28 & 1.09 & 0.74 \\
\hline N326 & Targeted & 0.0215 & 0.0732 & 0.0524 & -0.1306 & 1.05 & 1.18 & 1.13 & 0.74 & 0.0302 & 0.1120 & 0.0450 & -0.1155 & 1.07 & 1.29 & 1.11 & 0.77 \\
\hline
\end{tabular}




\subsubsection{PCT RESUlts FOR THE GLASSES REPRESENTING THE UNDERWASHED SLUDGE}

Exhibit E14 in Appendix E provides plots of the leachate concentrations and standards in the analytical sequence reported by the SRTC-ML for the PCTs from the underwashed case. These plots include the values from the EA PCTs and the blanks. These values expand the scales of these plots, making it difficult to distinguish among the results of the other analyses. Exhibit E15 in Appendix E provides these same plots excluding the EA and blank, yielding a clearer picture of the behavior of the PCTs for the other glasses and standards.

Exhibit E16 in Appendix E provides plots of the leachate concentrations for each type of submitted solution: the standards, the blanks, EA, ARM, and the study glasses representing the underwashed case. Once again, excluding the results for EA and the blanks improves the opportunity for investigating the behavior of the PCTs for the other glasses and standards. Exhibit E17 in Appendix E shows such results.

As a preliminary step to normalizing the PCTs from the underwashed case, statistical analyses were conducted on the results from the three analyses of the multi-element standard solution per analytical block. Exhibit E18 in Appendix E provides these analyses. Although there appears to be statistical differences among the block averages for most of the elements of interest, no bias correction of the PCT results for the study glasses was conducted. This approach was taken since the triplicate PCTs for a single study glass were placed in different ICP blocks. Averaging the ppm's for each set of triplicates helps to minimize the impact of the ICP effects.

Table 17 presents the block averages of the solution standards, and they indicate consistent and reasonably accurate results (differences of overall averages versus reference values $\sim 5 \%$ ) from these analyses. 
Table 17. Average Measurements of Standard Solution By Analytical Block - PCTs for Glasses from Underwashed Sludge.

\begin{tabular}{|c|c|c|c|c||}
\hline \multirow{2}{*}{$\begin{array}{c}\text { Analytical } \\
\text { Block }\end{array}$} & \multicolumn{4}{|c|}{ Average } \\
\cline { 2 - 5 } & B (ppm) & Li (ppm) & Na (ppm) & Si (ppm) \\
\hline \hline 1 & 18.9 & 9.5 & 79.3 & 47.9 \\
\hline 2 & 18.5 & 9.2 & 76.9 & 47.4 \\
\hline 3 & 18.7 & 9.3 & 78.3 & 47.5 \\
\hline 4 & 18.7 & 9.3 & 79.5 & 46.9 \\
\hline 5 & 19.2 & 9.4 & 77.9 & 48.4 \\
\hline 6 & 19.0 & 9.4 & 79.2 & 45.5 \\
\hline Grand Average & 18.8 & 9.3 & 78.5 & 47.3 \\
\hline Reference Value & 20 & 10 & 81 & 50 \\
\hline \% difference & $-5.9 \%$ & $-6.7 \%$ & $-3.1 \%$ & $-5.5 \%$ \\
\hline
\end{tabular}

The PCTs for the underwashed case were normalized (in a manner similar to the nominallywashed case discussed above) using the measured, measured bias-corrected, and the targeted compositions for the glasses from the underwashed case. Table 18 provides the results from the normalization process using the information in Table 15 and all of the data of Table E4 (i.e., before screening the PCT results for solution-weight problems). Exhibit E19 in Appendix E provides scatter plots for these results (both quenched and centerline cooled) offering an opportunity to investigate the consistency in the leaching across the elements for the glasses of this study. The consistency is typically demonstrated by a high degree of linear correlation among the values. PCT values normalized using targeted, measured, and bias-corrected compositions were investigated. A high degree of correlation is seen for these data for some pairs of elements. However, small correlations (as small as 49\%) can be seen between the data for some pairs of elements (e.g., in particular between $\mathrm{B}$ and $\mathrm{Na}$ as well as $\mathrm{Na}$ and $\mathrm{Li}$ ). 
Table 18. Normalized PCTs before Screening for

\section{Solution-Weight Problems - Underwashed Sludge.}

\begin{tabular}{|c|c|c|c|c|c|c|c|c|c|c|c|c|c|c|c|c|c|}
\hline \multirow[b]{2}{*}{\begin{tabular}{|c|} 
Glass \\
ID \\
\end{tabular}} & \multirow[b]{2}{*}{ Composition } & \multicolumn{8}{|c|}{ Quenched } & \multicolumn{8}{|c|}{ Centerline Cooled } \\
\hline & & {$\left[\begin{array}{c}\log \mathrm{NL} \\
{[\mathrm{B}(\mathrm{g} / \mathrm{L})]}\end{array}\right.$} & $\begin{array}{c}\log \mathbf{N L} \\
{[\mathrm{Li}(\mathrm{g} / \mathrm{L})]}\end{array}$ & $\begin{array}{r}\log \mathbf{N L} \\
{[\mathrm{Na}(\mathrm{g} / \mathrm{L})]}\end{array}$ & $\left.\begin{array}{|c|}\log \mathbf{N L} \\
{[\mathrm{Si}(\mathrm{g} / \mathrm{L})]}\end{array}\right]$ & \begin{tabular}{|c|}
$\mathbf{N L}$ \\
$\mathbf{B}(\mathrm{g} / \mathrm{L})$ \\
\end{tabular} & \begin{tabular}{|c}
$\mathbf{N L}$ \\
$\mathbf{L i}(\mathrm{g} / \mathrm{L})$ \\
\end{tabular} & $\begin{array}{c}\mathrm{NL} \\
\mathrm{Na}(\mathrm{g} / \mathrm{L})\end{array}$ & $\begin{array}{c}\mathbf{N L} \\
\mathrm{Si}(\mathrm{g} / \mathbf{L}) \\
\end{array}$ & $\begin{array}{l}\log \mathbf{N L} \\
{[\mathrm{B}(\mathrm{g} / \mathrm{L})]}\end{array}$ & $\left.\begin{array}{c}\operatorname{log~NL} \\
{[\mathbf{L i}(\mathrm{g} / \mathrm{L})]}\end{array}\right]$ & \begin{tabular}{|c}
$\log \mathrm{NL}$ \\
{$[\mathrm{Na}(\mathrm{g} / \mathrm{L})]$}
\end{tabular} & $\left.\begin{array}{|c}\log \mathrm{NL} \\
{[\mathrm{Si}(\mathrm{g} / \mathrm{L})]}\end{array}\right]$ & $\begin{array}{c}\mathbf{N L} \\
\mathbf{B}(\mathrm{g} / \mathrm{L}) \\
\end{array}$ & $\begin{array}{c}\text { NL } \\
\mathbf{L i}(\mathrm{g} / \mathrm{L})\end{array}$ & $\begin{array}{c}\mathrm{NL} \\
\mathrm{Na}(\mathrm{g} / \mathrm{L})\end{array}$ & $\begin{array}{c}\mathbf{N L} \\
\mathbf{S i}(\mathrm{g} / \mathrm{L} \\
\end{array}$ \\
\hline ARM & $\begin{array}{l}\text { See }[8] \\
\end{array}$ & $\begin{array}{l}-0.2991 \\
\end{array}$ & -0.2178 & -0.2713 & $\mid-0.5380$ & 0.50 & $\begin{array}{l}0.61 \\
\end{array}$ & 0.54 & 0.29 & & & & & & & & \\
\hline EA & e [8] & .2254 & & & & 16.80 & & & & & & & & & & & \\
\hline U165 & & & & & & 1.04 & 1.04 & 1.21 & 66 & .0057 & 0.0150 & 0.0422 & -0.1919 & 0.99 & 1.04 & 1.10 & 0.64 \\
\hline U165 & & 229 & & & & 1.05 & 1.04 & & & & & & & & & & 0.63 \\
\hline U165 & & 0067 & & & & 1.02 & .03 & & .67 & -0.0155 & 0.0146 & & & 0.97 & 1.03 & 1.12 & 0.65 \\
\hline U200 & & 0749 & 0.0743 & & & 0.84 & & & & -0.1150 & -0.1047 & & & 0.77 & & 0.78 & 0.49 \\
\hline $\mathrm{U} 200$ & & 0705 & -0.0773 & & & 0.85 & 84 & 87 & 0.51 & -0.1106 & -0.1076 & -0.1026 & & 0.78 & 0.78 & 0.79 & 0.49 \\
\hline $\mathrm{U} 200$ & & & & & & 0.84 & 85 & & 0.51 & & & & & 0.77 & 0.79 & 0.80 & \\
\hline $\mathrm{U} 303$ & & & & & & 0.81 & 90 & & 48 & & -0.0947 & & & 0.73 & 0.80 & 0.50 & 0.47 \\
\hline U303 & & & & & & 0.83 & 0.90 & & 0.48 & & -0.0902 & & & 0.74 & 0.81 & .50 & 0.47 \\
\hline U303 & & & & & & 0.80 & & & & & & & & 72 & & .51 & 0.48 \\
\hline & & & & & & 1.05 & 24 & & 0.87 & & & & & 0.97 & 1.21 & 1.58 & 0.78 \\
\hline & & & & & & 1.06 & & & & & & & & & & 1.59 & .79 \\
\hline & & & & & & 1.03 & 23 & & 89 & & & & & 9 & & 1.63 & 0.80 \\
\hline & & & & & & 1.05 & & & & & & & & & & 0.94 & \\
\hline U307 & & & & & & 1.07 & 1.50 & & 85 & 5790 & & & & & & 9.96 & 0.72 \\
\hline & & & & & & 1.03 & & & & & & & & & & 0.97 & 0.73 \\
\hline U313 & & & & & & & & & & & & & & & & 1.73 & 1.58 \\
\hline & & & & & & & 54 & & & & & & & & & .75 & .55 \\
\hline U31 & & & & & & 17 & 1.51 & & & & 4419 & & & 1.5 & & 1.73 & 1.60 \\
\hline U3 & & & & & & 1. & 34 & & & & & & & & & 0.77 & 0.38 \\
\hline U314 & & & & & & & & & & & & & & & & 0.79 & 0.38 \\
\hline & & & & & & 1. & 1.21 & & & & & & & & & 0.75 & 0.41 \\
\hline & & & & & & 1.32 & & & & & & & & & & .80 & 0.64 \\
\hline & & & & & & & & & & & & & & & & & .63 \\
\hline & & & & & & & & & & & & & & & & 94 & .63 \\
\hline & & & & & & & & & & & & & & & & 1.17 & 0.77 \\
\hline $\mathrm{U} 320$ & & & & & & & & & & & & & & & & & \\
\hline & & & & & & 0.99 & & & & & & & & 0.94 & & 1.19 & 0.79 \\
\hline & & & & & & & & & & & & & & & & & 0.43 \\
\hline U322 & & & & & & 0.55 & & & & & & & & & & & 0.44 \\
\hline U322 & & & & & & & & & & & & & & & & & \\
\hline \begin{tabular}{|l|} 
U323 \\
\end{tabular} & & & & & & & & & & & & & & 0.75 & & 0.63 & 0.44 \\
\hline & & & & & & & & & & & & & & & & & \\
\hline U32 & & & & & & & & & & & & & & & & & 45 \\
\hline \begin{tabular}{|l|} 
U324 \\
\end{tabular} & & & & & & & & & & & & & & 0.92 & & 71 & 0.54 \\
\hline & & & & & & & & & & & & & & & & & .55 \\
\hline \begin{tabular}{|l|} 
U324 \\
\end{tabular} & & 0056 & 0.0464 & -0.1078 & -0.2264 & 1.01 & & & & -0.0501 & -0.0100 & & & 0.89 & & 0.74 & 0.56 \\
\hline & & & & & & & & & & & & & & & & & 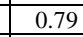 \\
\hline \begin{tabular}{|l|} 
U326 \\
\end{tabular} & Meas & 0572 & 0.0891 & 0.1315 & -0.1062 & 1.14 & 1.23 & & & 0.0629 & & 0.0992 & -0.0967 & & & 1.26 & 0.80 \\
\hline \begin{tabular}{|l|} 
U326 \\
\end{tabular} & Targeted & & 0.0848 & 0.1348 & -0.1059 & 1.11 & 1.22 & 1.36 & 0.78 & 0051 & 0.1100 & 0.1025 & -0.0965 & 1.13 & 1.29 & 1.27 & 0.80 \\
\hline
\end{tabular}


Table 19 compares the common logarithms of the leachate concentrations (in ppm) for the unscreened and screened PCTs where the screening was for the water-loss problem as discussed earlier; it also provides sludge representations. These results demonstrate that for these PCTs there were no significant differences in the screened and unscreened results. In this discussion that follows, all of the PCT results have been used to calculate the values of interest (i.e., the unscreened values were used).

Table 19. Average Leachate Concentrations from Screened and Unscreened PCTs.

\begin{tabular}{|c|c|c|c|c|c||}
\hline Glass ID & \# Used in & \multicolumn{5}{|c||}{ Mean } \\
\cline { 4 - 6 } (shortened) & Calculations & log[B ppm] & log[Li ppm] & log[Na ppm] & log[Si ppm] \\
\hline N165q & 3 & 1.350 & 1.421 & 2.091 & 2.179 \\
\hline N165q & 2 & 1.347 & 1.419 & 2.088 & 2.178 \\
\hline N200q & 3 & 1.330 & 1.193 & 1.875 & 2.081 \\
\hline N200q & 2 & 1.320 & 1.183 & 1.863 & 2.071 \\
\hline N307clc & 3 & 1.403 & 1.615 & 1.666 & 2.226 \\
\hline N307clc & 2 & 1.408 & 1.619 & 1.670 & 2.226 \\
\hline N314clc & 3 & 1.613 & 1.348 & 1.637 & 1.966 \\
\hline N314clc & 2 & 1.611 & 1.344 & 1.642 & 1.962 \\
\hline U165q & 2 & 1.376 & 1.405 & 2.144 & 2.210 \\
\hline U165q & 3 & 1.371 & 1.399 & 2.137 & 2.206 \\
\hline U303clc & 1 & 1.512 & 1.450 & 1.294 & 2.051 \\
\hline U303clc & 3 & 1.524 & 1.460 & 1.300 & 2.058 \\
\hline U313clc & 2 & 1.388 & 2.008 & 2.175 & 2.596 \\
\hline U313clc & 3 & 1.391 & 2.012 & 2.180 & 2.617 \\
\hline \hline
\end{tabular}

\subsubsection{PCT Results Versus Model Predictions}

The PCT response is a measure of the critical product quality metric for vitrified HLW - the durability of the glass. A review of Tables 16 and 18 reveals that the durabilities (as reflected by the PCTs) of the MB3 glasses compare very favorably to the durability of the EA glass. DWPF utilizes models to predict PCT responses based upon glass compositions. These models relate 
PCT response to glass compositions via an approach based on free energy of hydration (Jantzen et al. 1995).

The predictability of the PCT's of the MB3 glasses by these models is of concern. Exhibits E20 through E25 in Appendix E address this concern. Each of these exhibits presents a set of plots covering the four PCT elements of interest: boron (B), lithium (Li), sodium (Na), and silicon ( $\mathrm{Si}$ ). Each plot relates $\log \mathrm{NL}[\mathrm{x}(\mathrm{g} / \mathrm{L})]$ (where $\mathrm{x}$ represents $\mathrm{B}, \mathrm{Li}, \mathrm{Na}$, or $\mathrm{Si}$ ) to a linear function of $\Delta \mathrm{G}_{\mathrm{p}}$ (also represented as del $\mathrm{G}_{\mathrm{P}}$, a measured of the free energy of hydration in units of kcal/100 grams of glass) (Jantzen et al. 1995). Also, each plot shows the linear model surrounded by two lines that form a 95\% prediction interval for an individual PCT response corresponding to a $\Delta \mathrm{G}_{\mathrm{p}}$ value. Thus, one would expect a large portion of the PCT responses to fall within these prediction limits. The exhibits are organized around two features: the nominal versus underwashed cases and the composition view (measured, measured bc, and targeted).

Two final comments regarding these exhibits are warranted. The quenched and clc glasses were plotted using a closed, small square and an open circle, respectively. The EA and ARM results are shown (and labeled) on each plot.

Overall, the PCT's appear to be reasonably well predicted. The model underpredicted some of the PCT's at the more positive $\Delta \mathrm{G}_{\mathrm{p}}$ values. This behavior has been seen and documented in prior studies (Harbour et al. 2000). One or two glasses (e.g., N313 and N307) reveal predictable PCT responses for their quenched versions while their clc versions are just above the prediction limits. The next section provides a closer look at the quenched versus clc results.

\subsubsection{QUENCHED VERSUS CENTERLINE COOLED PCTS}

An important objective of this study was to investigate the potential impact of cooling rate on glass durability. Exhibits E26 (nominal-washed case) and E27 (underwashed case) provide a statistical comparison between the PCTs for the quenched and centerline cooled glasses. These exhibits show no statistically significant difference between the quenched and clc PCTs for either the nominally-washed or the underwashed cases. 
Figure 2 provides a series of scatter plots for the quenched versus clc PCTs for the nominally washed case. A plot is provided for each of the four elements along with a diagonal line as well as a fitted line for the average difference in the PCTs and 95\% confidence limits for this average. Figure 3 provides a similar series of scatter plots for the quenched versus clc PCTs for the underwashed case. In both figures, the nominal and underwashed MB3 glasses produced with Frit 313 appear to be outliers. The quenched versions of these glasses have a lower release than their counterpart clc versions. To identify potential sources for this difference, the nominal and underwashed Frit 313 glasses (both quenched and clc) were submitted to the SRTC-Analytical Development Section (ADS) for XRD analysis. Figures 4 and 5 represent the XRD results for the quenched and clc glasses using the nominally washed MB3 sludge and Frit 313. Figures 6 and 7 represent the XRD results for the quenched and clc glasses using the underwashed MB3 sludge and Frit 313.

XRD patterns of MB3N313q and MB3U313q (quenched) show the characteristic high background devoid of crystalline spectral lines indicative of an amorphous (non-crystalline) product. The absence of distinct spectral lines does not eliminate the possibility of amorphous phase separation in these glasses. For the clc versions of these glasses (see Figures 4 and 6), $\mathrm{Li}_{2} \mathrm{SiO}_{3}$ was detected in the glass (as noted by the well-defined or distinct spectral lines).

Although no formal analysis has been completed, the presence of amorphous phase separation in the quenched glasses and the formation of $\mathrm{Li}_{2} \mathrm{SiO}_{3}$ (during clc) may have led to the higher releases shown in Figures 2 and 3. It is known that amorphous phase separation can be a precursor to devitrification (Tomozawa 1972; Peeler and Hrma 1996) 
Fit of $\log \mathrm{NL}[\mathrm{B}(\mathrm{g} / \mathrm{L})]$ clc By $\log \mathrm{NL}[\mathrm{B}(\mathrm{g} / \mathrm{L})]$ quenched

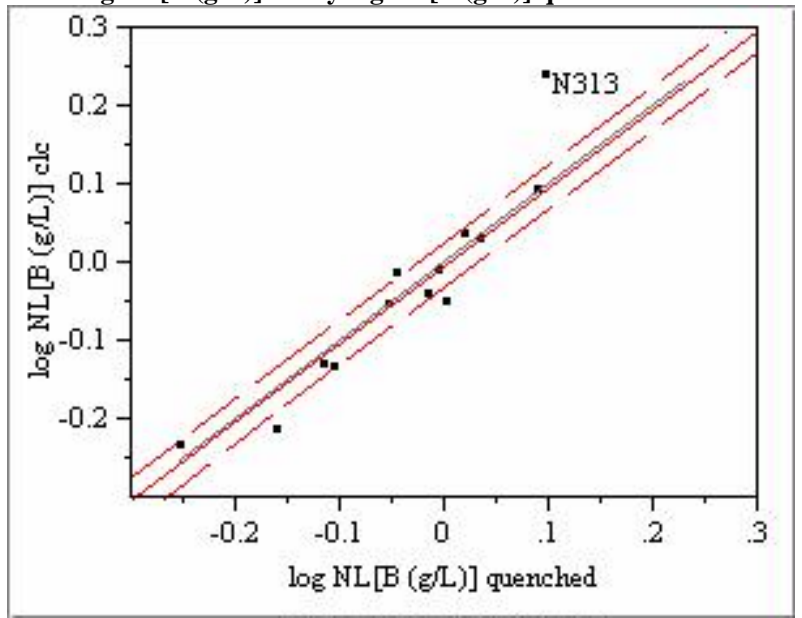

Fit of $\log N L[\mathrm{Li}(\mathrm{g} / \mathrm{L})]$ clc By $\log \mathrm{NL}[\mathrm{Li}(\mathrm{g} / \mathrm{L})]$ quenched

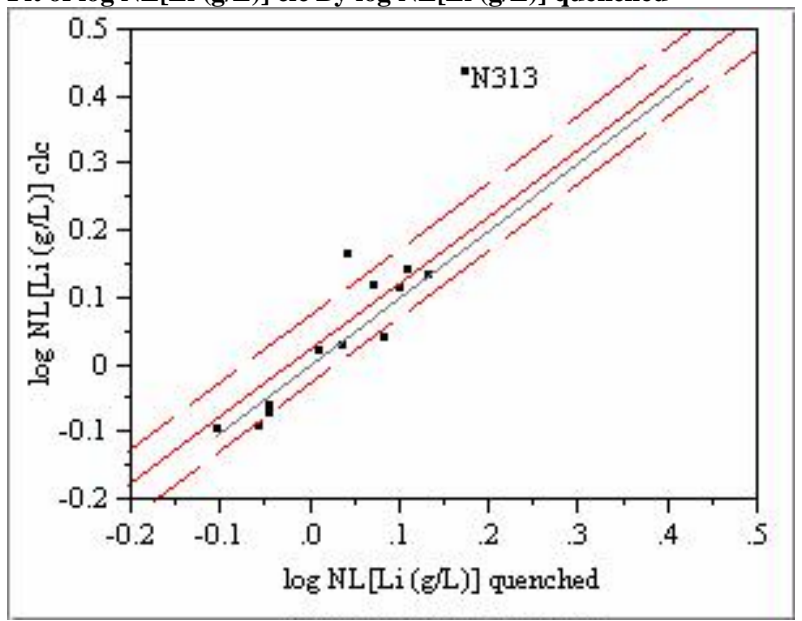

Fit of $\log \mathrm{NL}[\mathrm{Na}(\mathrm{g} / \mathrm{L})]$ clc By $\log \mathrm{NL}[\mathrm{Na}(\mathrm{g} / \mathrm{L})]$ quenched

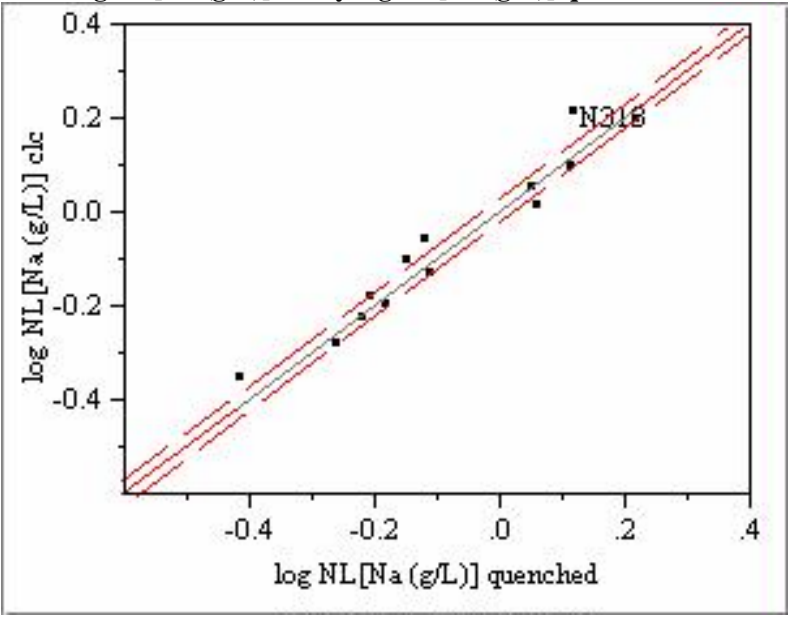

Fit of $\log \mathrm{NL}[\mathrm{Si}(\mathrm{g} / \mathrm{L})]$ clc By $\log \mathrm{NL}[\mathrm{Si}(\mathrm{g} / \mathrm{L})]$ quenched

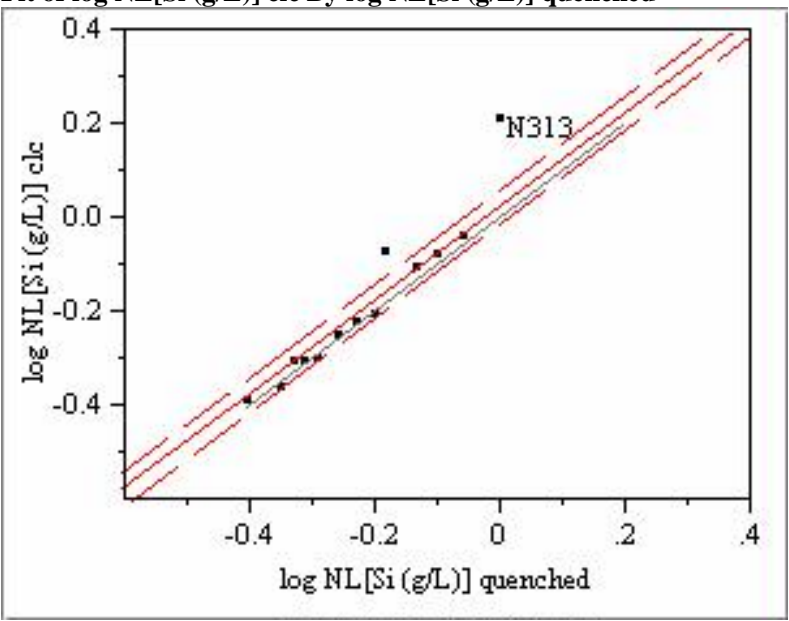

Figure 2. Quenched versus CLC PCTs for Nominally Washed Case. 
Fit of $\log \mathrm{NL}[\mathrm{B}(\mathrm{g} / \mathrm{L})]$ clc By $\log \mathrm{NL}[\mathrm{B}(\mathrm{g} / \mathrm{L})]$ quenched

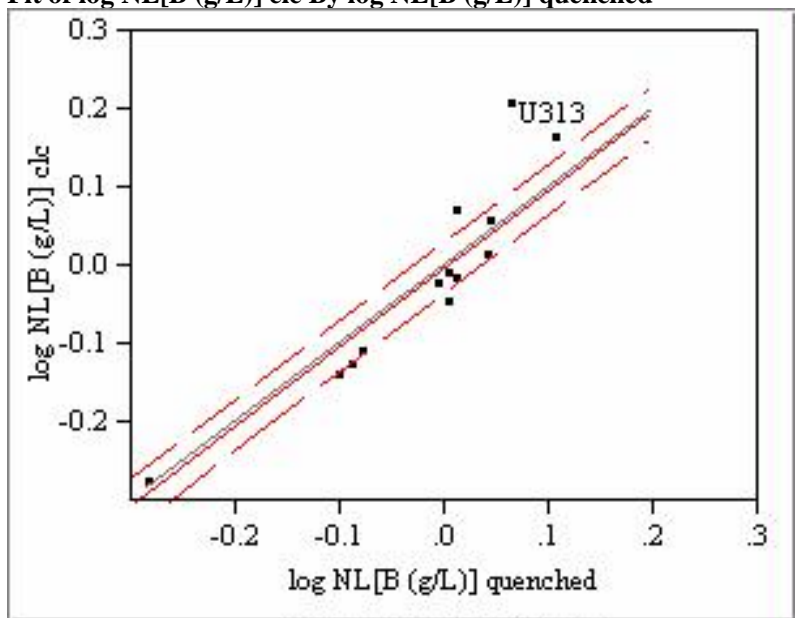

Fit of $\log \mathrm{NL}[\mathrm{Li}(\mathrm{g} / \mathrm{L})]$ clc By $\log \mathrm{NL}[\mathrm{Li}(\mathrm{g} / \mathrm{L})]$ quenched

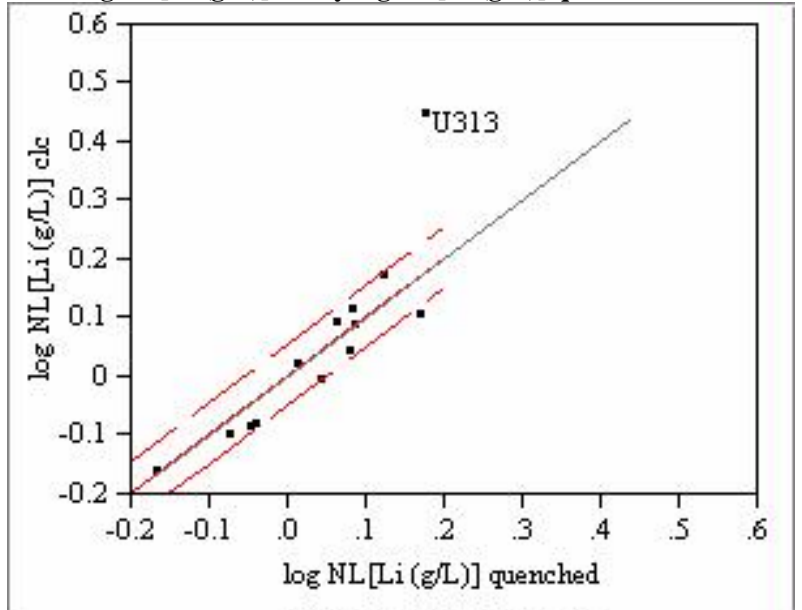

Fit of $\log \mathrm{NL}[\mathrm{Na}(\mathrm{g} / \mathrm{L})]$ clc By $\log \mathrm{NL}[\mathrm{Na}(\mathrm{g} / \mathrm{L})]$ quenched

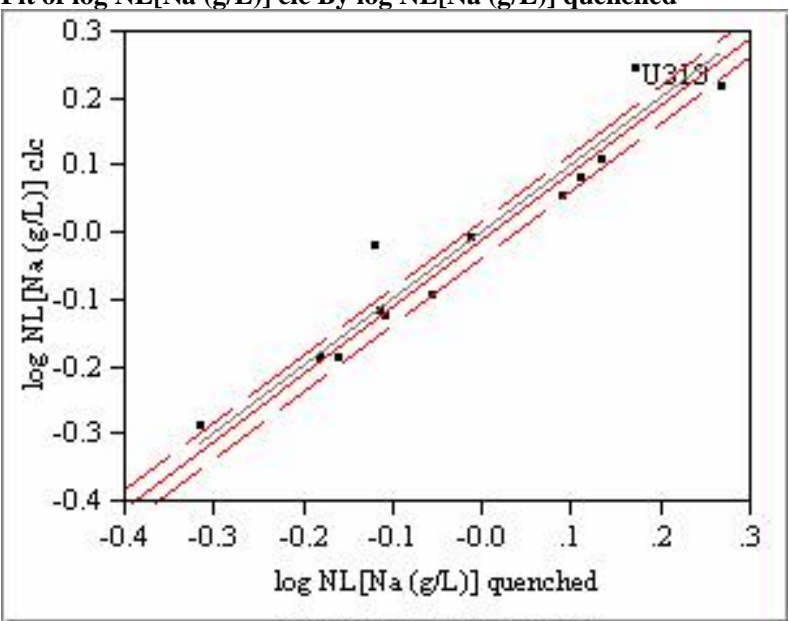

Fit of $\log \mathrm{NL}[\mathrm{Si}(\mathrm{g} / \mathrm{L})]$ clc By $\log \mathrm{NL}[\mathrm{Si}(\mathrm{g} / \mathrm{L})]$ quenched

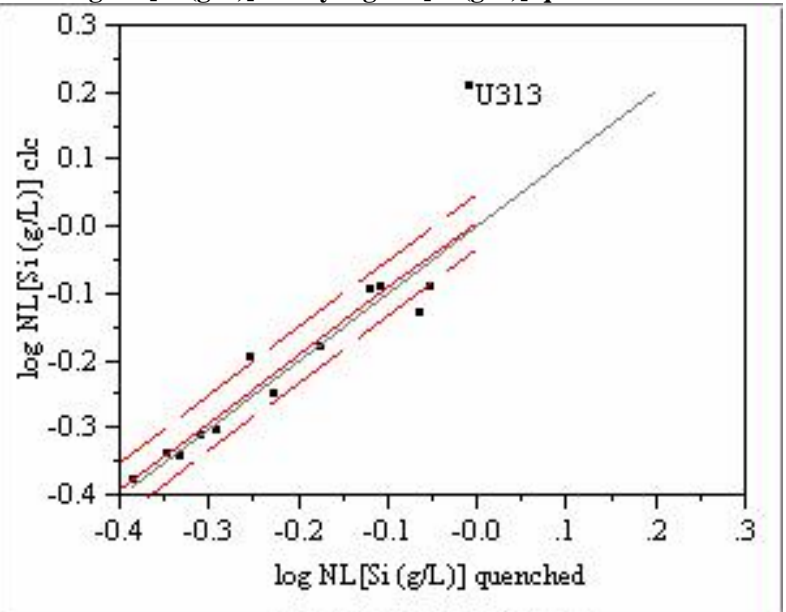

Figure 3. Quenched versus CLC PCTs for Underwashed Case. 


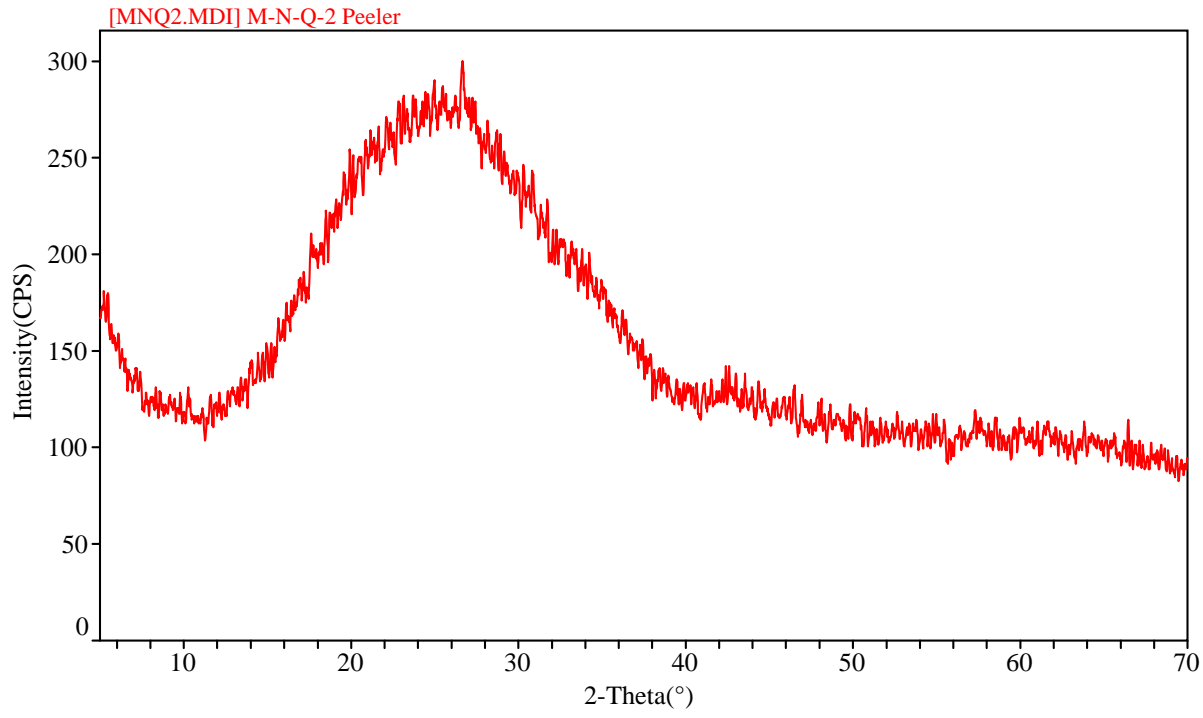

Figure 4. XRD Results of the MB3N313q.

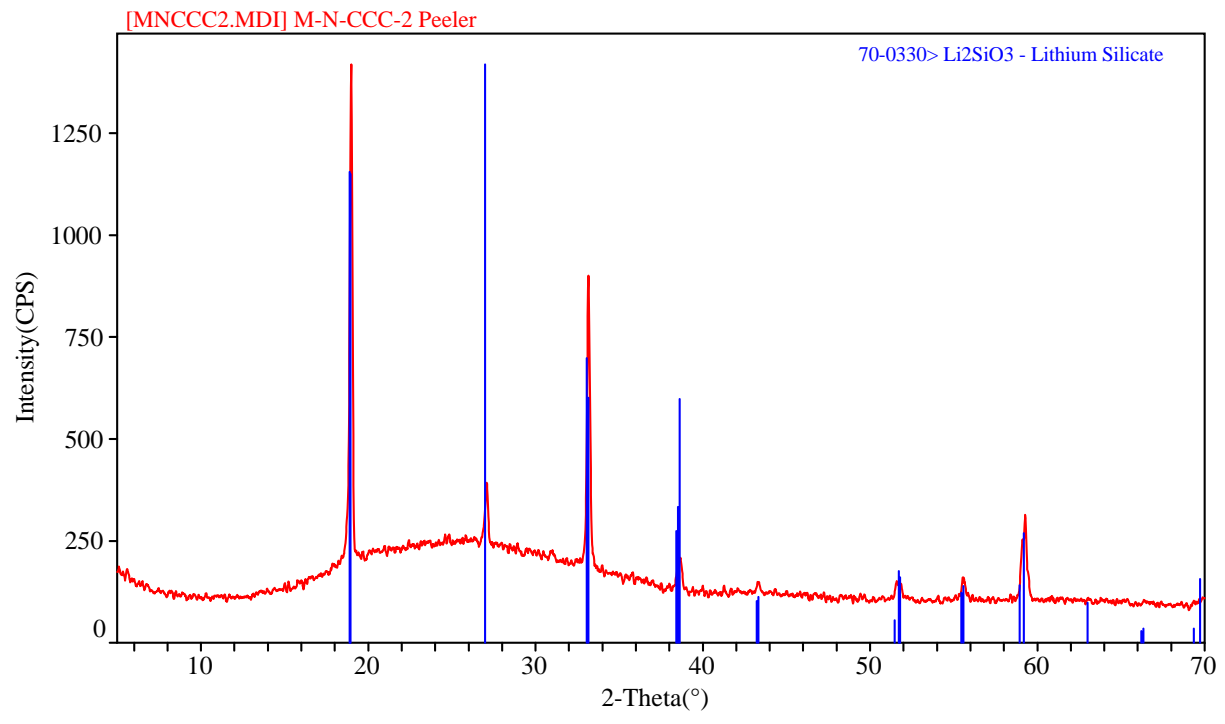

Figure 5. XRD Results of MB3N313clc. 


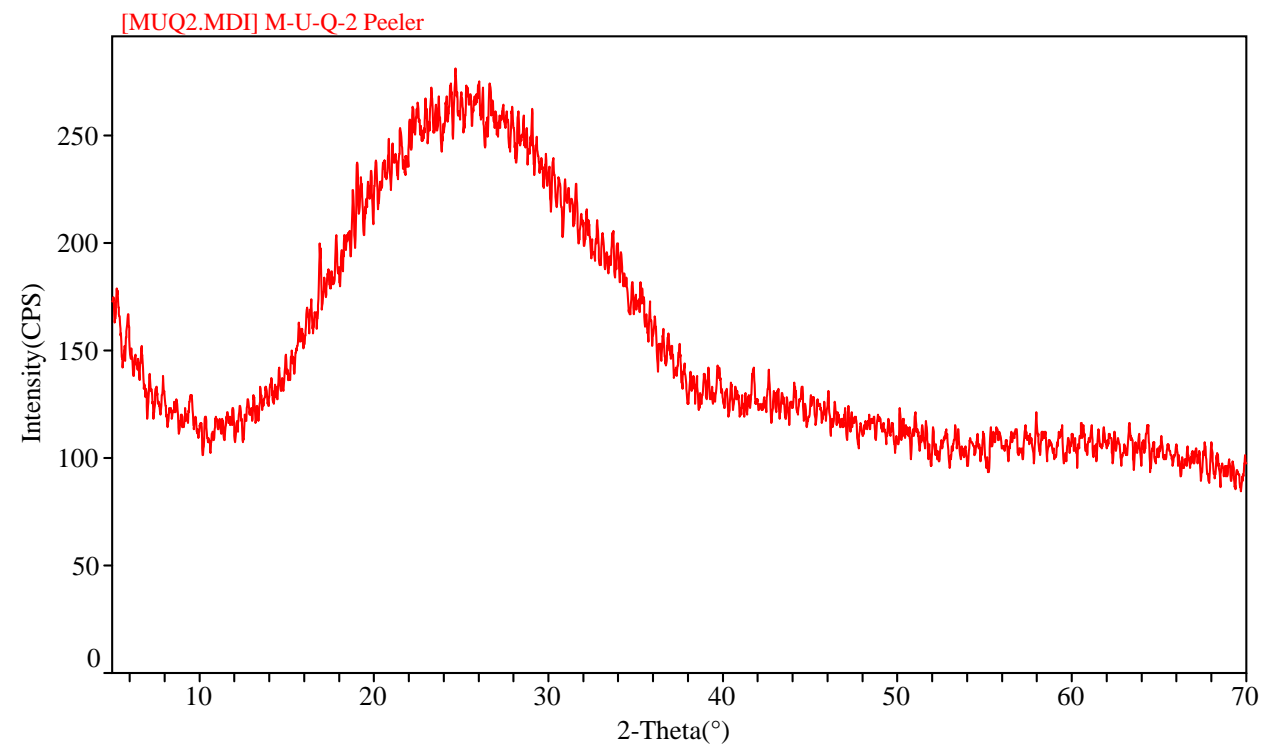

Figure 6. XRD Results of the MB3U313q.

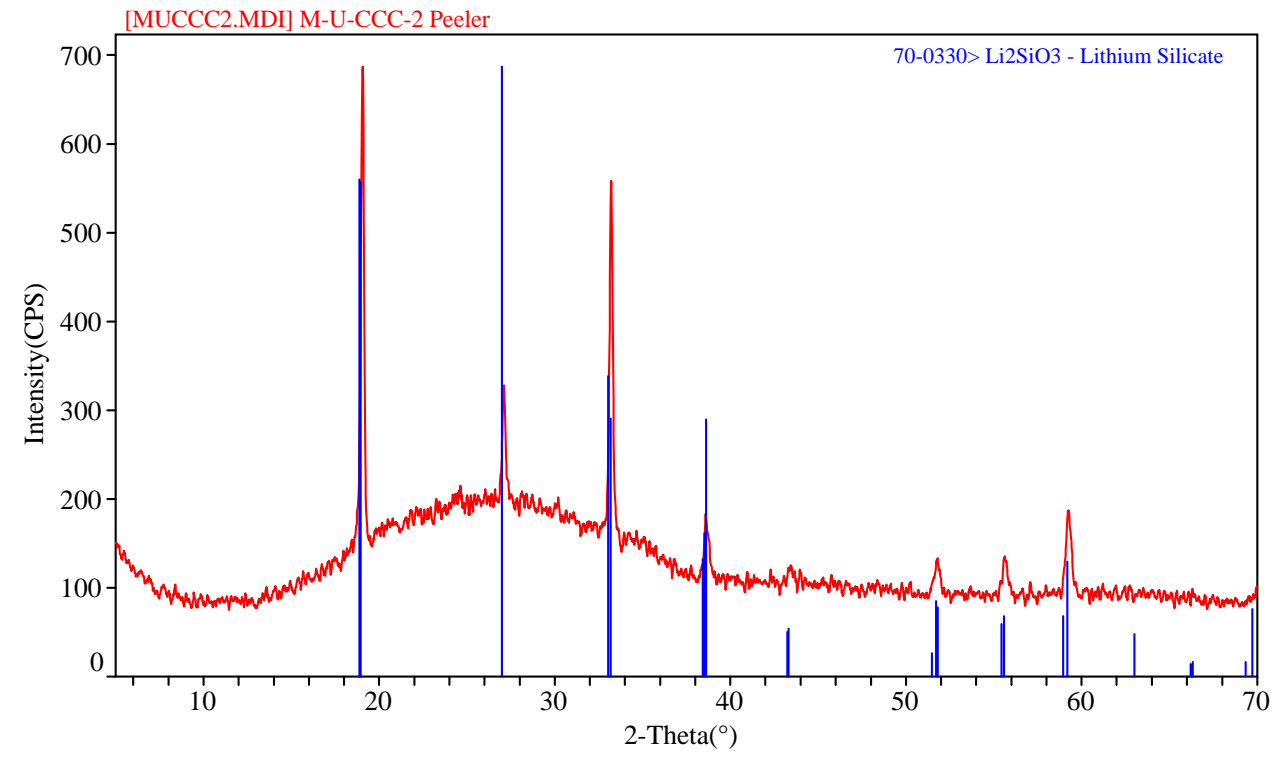

Figure 7. XRD Results of MB3U313clc. 


\subsection{VISCOSITY}

Table 19 summarizes the measured and predicted (via PCCS $\eta$ model) high temperature $\eta$ data for 12 MB3 glasses. Six unique frit compositions were used coupled with both the nominal and underwashed sludge at $25.5 \mathrm{wt} \%$ loading. Section 7.1 discusses target and measured chemical compositions of these glasses. The Batch 1 standard was measured to be 48.3 Poise at $1150^{\circ} \mathrm{C}$ which is consistent with that reported by Schumacher and Peeler (1998). General trends in the data indicate that for a given frit composition the $\eta$ of the underwashed sludge is slightly lower relative to its nominal sludge counterpart. This is primarily a result of the additional $\mathrm{Na}_{2} \mathrm{O}$ in the underwashed sludge (refer to Table 1, Section 4.0). All glasses meet the current $20-100$ Poise processing criteria for acceptability. However, the viscosities of these glasses are lower relative to those previously processed through DWPF. This may be advantageous with respect to melt rate given convection currents control the batch - melt interface by minimizing foam formation and/or stability.

Table 19. Measured and Predicted $\eta_{1150^{\circ} \mathrm{C}}$ (in Poise) of Select MB3 Glasses.

\begin{tabular}{|c|c|c|}
\hline Glass & $\eta_{1150^{\circ} \mathbf{C}}$ Poise (measured) & $\eta_{1150^{\circ} \mathbf{C}}$ Poise (predicted) \\
\hline \hline MB3N304 & 43.05 & 32.4 \\
\hline MB3U304 & 41.07 & 30.3 \\
\hline MB3N307 & 32.88 & 41.1 \\
\hline MB3U307 & 29.24 & 38.7 \\
\hline MB3N313 & 35.31 & 37.6 \\
\hline MB3U313 & 34.08 & 35.5 \\
\hline MB3N320 & 38.55 & 40.7 \\
\hline MB3U320 & 38.02 & 38.3 \\
\hline MB3N324 & 44.64 & 53.3 \\
\hline MB3U324 & 42.07 & 50.1 \\
\hline MB3N165 & 34.86 & 33.1 \\
\hline MB3U165 & 34.51 & 31.1 \\
\hline
\end{tabular}




\subsection{SME ACCEPTABILITY: OPTIONS FOR IMPROVING MELT RATE}

The determination as to whether a candidate frit will improve melt rate relative to Frit 200 (assumed to be the baseline case in this study) cannot be made based solely on the model assessments or the limited data discussed in this report. It must be reiterated that models that allow a direct assessment of melt rate do not exist. Lorier (2001) and Stone and Josephs (2001) compared melt rates (based on experimental data using a suite of tests) for the glasses developed in this study. The information presented in this report could, however, provide input to the selection process for those glasses that have been shown to have an improved melt rate relative to the current Frit 200 baseline. The decision as whether or not to select a candidate frit (with respect to durability) may be based on the answers to the following series of questions:

(1) Does the glass "pass" the current SME acceptability durability criteria (Brown and Postles 1996)? That is, does the glass, based on a measured composition, have a predicted $\Delta \mathrm{G}_{\mathrm{P}}>$ 12.72 (i.e., the most conservative of the element release limits at the PAR)?

(2) Does the model predict the PCT well? That is, does the measured PCT result lie within the 95\% confidence intervals for individual PCT results?

(3) How does the release for the candidate frit compare to EA?

To demonstrate how the model assessments could impact the decision process, consider the following scenario. Figure 8 is a plot of the DWPF durability model that relates the logarithm of the normalized PCT (in this case for boron) to a linear function of a free energy of hydration term ( $\Delta \mathrm{G}_{\mathrm{P}}, \mathrm{kcal} / 100 \mathrm{~g}$ glass) derived from the glass composition. Prediction limits (represented by the dashed lines) at 95\% confidence for individual PCT results are also shown around this linear fit. The position of each glass (based on a $25.5 \mathrm{wt} \%$ MB3 loading (with uranium) using a nominal wash scenario) in Figure 8 is based on measured data (both composition and PCT release).

First consider glasses produced from Frit 200 and Frit 165 using MB3 at 25.5 wt $\%$ loading. Both glasses would "pass" the initial questions regarding the SME acceptability durability criterion. The $\Delta \mathrm{G}_{\mathrm{P}}$ values for MB3N200 and MB3N165 (at $25.5 \mathrm{wt} \%$ loading) are approximately -10.45 and -12.25 , respectively. Both glasses fall within the $95 \%$ confidence intervals indicating that the PCTs are well predicted by the current durability model (addressing the second question). Their respective B release values are also well below the EA release values making them "acceptable". 


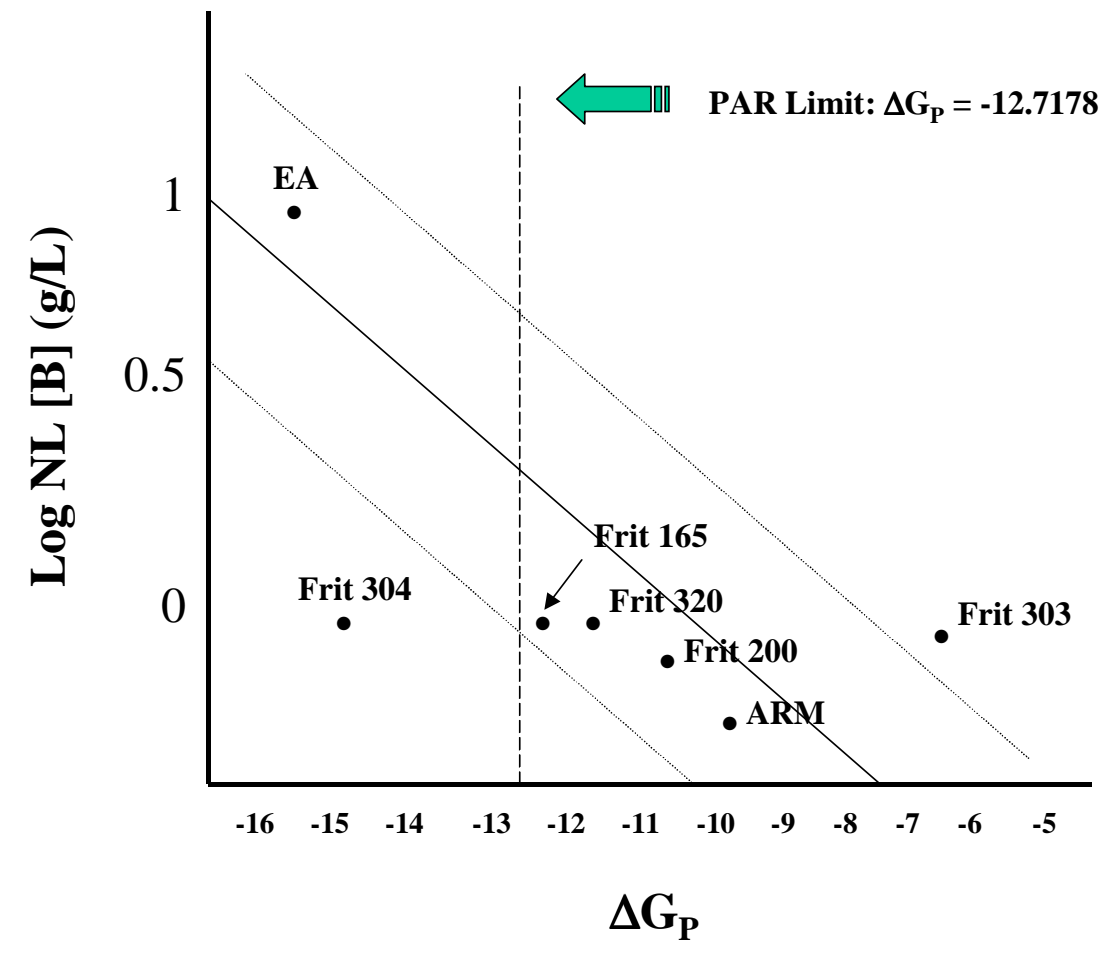

Figure 8. Schematic of $\Delta G_{P}$ Versus Log NL [B] (g/L) for Various MB3 Glasses.

(although nomenclatures for frits are used the PCT results are based on MB3 glasses produced from these frits)

Assuming that the use of Frit 200 does impede melt rate for MB3, Frit 165 appears to be a viable candidate to use with nominally washed MB3 sludge. However, if an underwashed sludge is received in DWPF, the use of Frit 165 could push the durability acceptability criterion to the edge. Based on the PCCS model predictions shown in Table 10 (again recognizing that the minor components have been normalized out), the projected $\Delta \mathrm{G}_{\mathrm{P}}$ (based on the target composition) is 12.69 with the SME acceptability limit being -12.72 at the PAR (see Table 11 for PAR limits.) Selecting Frit 165 may be a high risk because the $\Delta \mathrm{G}_{\mathrm{P}}$ prediction is based on a target glass composition, and the uncertainties associated with actual MB3 sludge composition, washing efficiencies, and/or waste loadings have not been accounted for. The latter statement is based on the assumption that, if melt rate improvements are such that Frit 165 is a viable candidate, the required variability study may indicate compositional areas in which the initial SME acceptability criteria would limit processing. It does appear that Frit 165 defines the bounds in terms of challenging the current $\Delta \mathrm{G}_{\mathrm{P}}$ limit while remaining within the prediction intervals. If Frit 165 is 
demonstrated to have a significant improvement in melt rate, any negative ramification encountered in the variability study (e.g., creating a "no go" situation in terms of SME acceptability over some portion of the composition region) could be addressed using an alternative acceptability method (as described in the discussion below for Frit 304).

Next consider the glass (e.g., MB3N320) produced from Frit 320. Assuming this glass demonstrated an improved melt rate relative to Frit 200, there appears to be no negative impacts of selecting of this glass in terms of the SME acceptability durability criterion. That is, this glass "passes" the $\Delta \mathrm{G}_{\mathrm{P}}$ criteria with a predicted $\Delta \mathrm{G}_{\mathrm{P}}$ of -11.77 (based on measured composition and a nominally washed sludge). The glass lies within the $95 \%$ confidence intervals indicating the PCT is well predicted, and its measured PCT values are well below that of EA. If an underwashed sludge is delivered to DWPF, Frit 320 would still be an "acceptable candidate" from the SME durability criterion perspective $\left(\Delta \mathrm{G}_{\mathrm{P}}=-12.22\right.$ for the underwashed sludge case).

A glass (e.g., MB3N303) made from Frit 303 is another option to consider. Based on $\Delta \mathrm{G}_{\mathrm{P}}$ predictions, this glass would be well above the -12.72 PAR acceptability limit with a $\Delta \mathrm{G}_{\mathrm{P}}$ of -6.33 (based on a nominal washed MB3 sludge target composition). However, its prediction lies outside the $95 \%$ confidence intervals indicating that the model does not predict its PCT release value well. Historically, these glasses have been "acceptable", however, given the fact that their release values are well below that of EA. Assuming Frit 303 shows a marked improvement in melt rate over that of Frit 200 (for MB3), its selection would not pose a high risk in terms of the SME acceptability criteria that would be subsequently assessed in a variability study. It should be noted that Frit 303 contains $20.13 \mathrm{wt} \% \mathrm{~B}_{2} \mathrm{O}_{3}$ and may be phase separated (amorphous).

The last case to consider (Frit 304) poses a more difficult challenge in terms of SME acceptability for durability. Glass produced from Frit 304 and MB3 "fails" the first two major hurdles for SME acceptability. That is, its predicted $\Delta \mathrm{G}_{\mathrm{P}}$ is more negative than the $-12.72 \mathrm{PAR}$ limit $\left(\Delta \mathrm{G}_{\mathrm{P}}=\right.$ -13.79 and -14.24 for the nominal and underwashed MB3 sludge cases, respectively) and it lies outside the $95 \%$ confidence intervals indicating that the model does not predict its PCT release well. However, prior to eliminating this frit from further consideration to improve melt rate for MB3, a review of the technical issues relative to measured PCT data (see Section 7.0) should be made. This is especially the case since the composition for this glass lies outside the region from which the model represented in Figure 8 was generated. This review or assessment could provide 
a basis for making the decision on whether Frit 304 poses an unacceptable technical risk to pursue as a candidate frit. The risk level one sets must be balanced by the potential gains in terms of melter throughput.

Table 20 summarizes the B, Na, Li, and Si normalized release (NR) values based on measured compositions for MB3N304 and MB3U304 (both quenched and clc). Although $\Delta \mathrm{G}_{\mathrm{P}}$ predictions would currently restrict DWPF from processing MB3N304 or MB3U304, the measured PCT data indicate that glasses produced from either the nominal or underwashed sludge (for both thermal heat treatments) are $<2 \mathrm{~g} / \mathrm{L}$ (for all reportable elementals) which are still well below that of EA (approximately $16.7 \mathrm{~g} / \mathrm{L}$ for B). The data also indicated no significant difference between the quenched and clc PCTs for either the nominally washed or the underwashed MB3 sludge cases.

Table 20. Normalized Release for Glasses Produced with MB3 and Frit 304 (Nominal and Underwashed Sludge).

\begin{tabular}{||c|c|c|c|c|c||}
\hline Glass & & NR [B] & NR [Li] & NR [Na] & NR [Si] \\
\hline MB3N304q & $\mathrm{g} / \mathrm{L}$ & 1.07 & 1.25 & 1.97 & 0.87 \\
\hline & Log g/L & 0.029 & 0.096 & 0.296 & -0.064 \\
\hline MB3N304clc & $\mathrm{g} / \mathrm{L}$ & 1.05 & 1.27 & 1.86 & 0.88 \\
\hline & Log g/L & 0.019 & 0.103 & 0.270 & -0.056 \\
\hline MB3U304q & $\mathrm{g} / \mathrm{L}$ & 1.05 & 1.24 & 1.81 & 0.87 \\
\hline & Log g/L & 0.023 & 0.092 & 0.257 & -0.062 \\
\hline MB3U304clc & $\mathrm{g} / \mathrm{L}$ & 0.97 & 1.21 & 1.58 & 0.78 \\
\hline & Log g/L & -0.013 & 0.084 & 0.199 & -0.105 \\
\hline
\end{tabular}

Assume that Frit 304 increases melt rate and its relative increase compared to Frit 200 (or other frits assessed) is such that one would consider Frit 304 as the primary candidate for MB3. It must be reiterated that the assessment of melt rate given the suite of tests or test methodology used is assumed to translate directly to DWPF (see Lorier (2001) and Stone and Josephs (2001) for a discussion of the results of these tests). Although the current durability model does not predict an acceptable release, the data presented in Table 20 indicate that all elemental releases are less than $2 \mathrm{~g} / \mathrm{L}$. 
If Frit 304 were selected as the primary candidate, one must develop an alternative technique that could be used by DWPF to address the SME acceptability issue. Alternative methods exist to derive constraints that would allow DWPF to utilize Frit 304. Alternatives include but are not limited to: (i) developing a non-parametric model over the composition region and/or (ii) revising the current DWPF durability model. Regardless of the pathway selected, the development of a technical foundation to support replacing the current durability acceptance criteria may be a nontrivial task. Although the options to address the technical issues with SME durability acceptance for Frit 304 are not trivial, the technical team does feel that they can and should be adequately addressed since this has promise to open up the operating window.

These options would have to be integrated with other studies such as the current effort on reducing constraints for sludge-only processing and/or the new $\mathrm{T}_{\mathrm{L}}$ modeling effort. In fact, the results of the reduction of constraints task aimed at relaxing constraints on durability could benefit this particular frit selection option.

The pathforward chosen will ultimately be influenced by the relative increase in melt rate that one frit has over the current baseline, the acceptable risk level one is willing to take, and/or budget/schedule restrictions. Based on this assessment, budget/schedule impacts would be minimized by the selection of a frit that "passes" the current SME acceptability criteria for durability. However, this path may result in a frit that does not maximize melt rate for MB3. If selection of Frit 304 is deemed unacceptable for MB3 (either due to an associated risk level or budget/schedule influences), one should continue to address the technical issues identified (e.g., model prediction of durability for Frit 304) at some level given that future sludge-only (or coupled) flowsheets may yield these same issues. If so, then frits can be rapidly developed and implemented while reducing technical risks. 


\subsection{SUMMARY}

The objective of this research was to enhance the basic understanding of the role of glass batch chemistry (more specifically via control of frit composition) on the overall melting process for Macrobatch 3 (MB3). Through control of the frit composition, cold cap reactions can be altered which may result in higher melter throughput. For melt rate limited systems, a small increase in melting efficiency translates into substantial savings by reducing operational costs without compromising the quality of the final waste form or product.

The overall strategy for the frit development activities was to explore frit compositional regions (both oxide components and ranges) which challenged "acceptable" predicted property behavior. Once major frit components were identified, ranges were established to push or challenge model predictions in an attempt to maximize melt rate. Twenty-seven frits were developed using various model predictions as a guide. All frits are projected to maintain an equivalent operational window in terms of waste loading range relative to the "baseline case" ( $25-28$ wt $\%$ MB3 with Frit 200) based on model predictions while hopefully increasing melt rate. Candidate frit compositions were screened to ensure that although melt rate may be improved other properties (e.g., durability, liquid, and $\eta$ ) are not compromised (i.e., the systems approach was applied).

To obtain a manageable set of candidate frits for which melt rate could be experimentally assessed within budget and schedule constraints, an initial selection process was used to narrow the 27 potential frit compositions down to 15 . Compositional guidelines established by SRTC researchers along with preliminary isothermal crucible tests were used in the down selection process. Glasses were fabricated at a target waste loading of $25.5 \mathrm{wt} \%$ and selected properties were measured. An important objective of this study was to investigate for a potential impact on glass durability (as defined by the PCT) due to cooling rate. The data indicated no statistically significant difference between the quenched and clc PCTs for either the nominally washed or the underwashed MB3 sludge cases.

The ultimate determination as to whether a candidate frit will improve melt rate relative to Frit 200 (assumed to be the baseline case in this study) can not be made based solely on the model assessments or limited data discussed in this report. Comparisons of melt rates (based on experimental data using a suite of tests) for the glasses developed in this study have been made by 
Lorier (2001) and Stone and Josephs (2001). Information is presented which provides input in the selection process for those glasses that are shown to have an improved melt rate relative to the current Frit 200 baseline.

The decision as to whether or not to select a candidate frit is influenced by the answers to the following series of questions:

(1) Does the glass "pass" the SME acceptability durability criteria? That is, does the glass, based on a measured composition, have a predicted $\Delta \mathrm{G}_{\mathrm{P}}>-12.72$ (the most conservative of the element release limits at the PAR)?

(2) Does the model predict the PCT well? That is, does the PCT lie within the $95 \%$ confidence intervals for individual PCT results?

(3) How does the release for the candidate frit compare to EA?

The majority of the frits developed "pass" the SME acceptability criteria (i.e., $\Delta \mathrm{G}_{\mathrm{P}}$ limit) and lie within the $95 \%$ prediction confidence interval indicating that the current model accurately predicts the PCTs. Selection of a glass in this category poses a minimum risk in terms of passing other predicted SME acceptability criteria (i.e., process and product performance properties). However, prior to DWPF implementation of a glass within this category, a variability study is required and other properties beyond durability should be assessed. One property of particular interest would be $\mathrm{T}_{\mathrm{L}}$ to ensure that the current (or future) model predictions do not limit the operational window and are applicable (i.e., within the same primary phase field).

A few of the MB3 glasses fall in the category of "passing" the SME acceptability criteria for durability (i.e., $\Delta \mathrm{G}_{\mathrm{P}}$ ) but lying outside the $95 \%$ interval at more positive $\Delta \mathrm{G}_{\mathrm{P}}$ (e.g., a MB3 glass produced from Frit 303). Historically, glasses in this category have been "acceptable" given the fact that their release values are well below that of EA. Selection of a glass in this category again poses a minimum risk in terms of passing other predicted SME acceptability criteria. However, prior to implementing a glass in this category, a variability study is required to evaluate durability over the projected compositional range.

A MB3 glass produced using Frit 304 falls into a third category: it "fails" the primary acceptance criterion based on current model predictions and it lies outside the $95 \%$ confidence interval. 
Although $\Delta \mathrm{G}_{\mathrm{P}}$ predictions would currently restrict DWPF from processing MB3N304 or MB3U304, the measured PCT data indicates that glasses produced from either the nominal or underwashed sludge (for both thermal heat treatments) have elemental releases $<2 \mathrm{~g} / \mathrm{L}$ which are still well below that of EA.

Assuming that Frit 304 increases melt rate and its relative increase compared to Frit 200 (or other frits assessed) is such that one would consider Frit 304 as the primary candidate for MB3, alternative technique(s) must be developed to address the SME acceptability criteria. Alternative pathways proposed include (but are not limited to): (i) developing non-parametric models over the composition regions, and/or (ii) enhancing the current DWPF durability model predictions. 


\subsection{REFERENCES}

American Society for Testing and Materials (ASTM). 1997. Standard Test Methods for Determining Chemical Durability of Nuclear Waste Glasses: The Product Consistency Test (PCT), ASTM C 1285-97, in Annual Book of ASTM Standards, Vol. 12.01, ASTM, West Conshohocken, Pennsylvania.

Brown, K. G. and T. B. Edwards. 1995. Definition of the DWPF Predictability Constraint (U), WSRC-TR-95-0060, Revision 0, Westinghouse Savannah River Company, Aiken, South Carolina.

Brown, K. G. and R. L. Postles. 1996. SME Acceptability Determination for DWPF Process Control (U), WSRC-TR-95-0364, Revision 3, Westinghouse Savannah River Company, Aiken, South Carolina.

Edwards, T. B. 2000. Statistical Analysis Supporting DWPF's Macrobatch 3 Variability Study (U), WSRC-RP-2000-00774, Rev. 0, Westinghouse Savannah River Company, Aiken, South Carolina.

Edwards, T. B., and K. G. Brown. 1998. Evaluating the Glasses Batched for the Tank 42 Variability Study (U), SRT-SCS-98-017, Rev. 0, Westinghouse Savannah River Company, Aiken, South Carolina.

Elder, H. H.. 2000. Position Paper on Sludge Batch 2 Qualification Strategy and Simulant Composition, HLW-SDT-2000-00128, High Level Waste Salt Disposition Systems Engineering Team, Rev. 0., Westinghouse Savannah River Company, Aiken, South Carolina.

Fowler, J. R., R. E. Edwards, S. L. Marra, and M. J. Plodinec. 1991. Chemical Composition Projections for the DWPF Product (U), WSRC-IM-91-116-1, Rev. 1, Westinghouse Savannah River Company, Aiken, South Carolina.

Harbour, J. R., T. B. Edwards, and R. J. Workman. 2000. Summary of Results for Macrobatch 3 Variability Study (U), WSRC-TR-2000-00351, Rev. 0, Westinghouse Savannah River Company, Aiken, South Carolina.

Hester, J. R. 1996. High Level Waste Characterization System, WSRC-TR-96-0264, Westinghouse Savannah River Company, Aiken, South Carolina.

Jantzen, C. M. 1986. Systems Approach to Nuclear Waste Glass Development, J. Non-Cryst Solids, 84 [1 - 3] 215 - 225 (1986).

Jantzen, C. M. 1988. Glass Composition and Frit Formulation Developed for DWPF, DPST-88952, E.I du Pont de Nemours and Company, Savannah River Laboratory, Aiken, South Carolina.

Jantzen, C.M., N. E. Bibler, D. C. Beam, C. L. Crawford and M. A. Pickett. 1993. Characterization of the DWPF Environmental Assessment (EA) Glass Standard Reference Material (U), WSRC-TR-92-346, Rev. 1, Westinghouse Savannah River Company, Aiken, South Carolina. 
Jantzen, C. M., J. B. Pickett, K. G. Brown, T. B. Edwards, and D. C. Beam. 1995. Process/Product Models for the Defense Waste Processing Facility (DWPF): Part I. Predicting Glass Durability from Composition Using a Thermodynamic Hydration Energy Reaction Model $\left(T H E R M O^{T M}\right)(U)$, WSRC-TR-93-673, Rev. 1, Volume 1, Westinghouse Savannah River Company, Aiken, South Carolina.

Jantzen, C.M., R.L. Schulz, D.K. Peeler, J.D. Vienna, and P.R. Hrma. 1999. Tanks Focus Area Technical Exchange Meeting: Crystallization and Amorphous Phase Separation in HLW Glasses (U), WSRC-RP-99-01067, Appendix E, Westinghouse Savannah River Company, Aiken, South Carolina.

Kim, D. S. and P. Hrma. 1994. "Laboratory Studies for Estimation of Melting Rate in Nuclear Waste Glass Melters." In: Ceramic Transactions, Environmental and Waste Management Issues in the Ceramic Industry II, Volume 45, pp. 409-419.

Lambert, D. P., T. H. Lorier, D. K. Peeler, and M. E. Stone. 2001. Melt Rate Improvement for DWPF MB3: Summary and Recommendations (U), WSRC-TR-2001-00148, Rev. 0, Westinghouse Savannah River Company, Aiken, South Carolina.

Li, H., J. D. Vienna, P. Hrma, P. Smith, D. E. Schweiger, and M.J. Schweiger. 1997. "Nepheline Precipitation in High-Level Waste Glasses: Compositional Effects and Impact on the Waste Form Acceptability, Scientific Basis for Nuclear Waste Management XX," 261-268, Materials Research Society, Pittsburgh, Pennsylvania.

Lorier, T. H. 2001. Melt Rate Improvement for DWPF MB3: Crucible Studies (U), WSRC-TR2001-00151, Rev. 0, Westinghouse Savannah River Company, Aiken, South Carolina.

Marra, S. L., and C. M. Jantzen. 1993. Characterization of Projected DWPF Glasses Heat Treated to Simulate Canister Centerline Cooling (U), WSRC-TR-92-142, Rev. 1 Westinghouse Savannah River Company, Aiken, South Carolina.

Peeler, D. K. and P. Hrma. 1996. "Compositional Range of Durable Borosilicate Simulated Waste Glasses." In: Emerging Technologies in Hazardous Waste Management VI, Chapter 21, American Academy of Environmental Engineers, pp. 323-338.

Peeler, D. K., T. H. Lorier, and J. D. Vienna. 2001. Improve Melt Rate for DWPF MB3: Foaming Theory and Mitigation Techniques (U), WSRC-RP-2001-00351, Rev. 0, Westinghouse Savannah River Company, Aiken, South Carolina.

Peeler, D. K., T. B. Edwards, K. G. Brown, R. J. Workman, and I. A. Reamer. 2000. Reduction of Constraints: Applicability of the Homogeneity Constraint for MB3 (U), WSRC-TR-200000358, Rev. 0, Westinghouse Savannah River Company, Aiken, South Carolina.

SAS Institute, Inc. 2000. JMP Statistics and Graphics Guide, Version 4, Cary, North Carolina.

Savannah River Technology Center (SRTC). 1996a. "Glass Batch Preparation Procedure." In: Glass Technology Manual L13.1, Technical Reference, GTOP-3-003, Rev. 4, Aiken, South Carolina. 
Savannah River Technology Center (SRTC). 1996b. "Glass Melting Procedure.” In: Glass Technology Manual L13.1, Technical Reference, GTOP-3-004, Rev. 4, Aiken, South Carolina.

Savannah River Technology Center (SRTC). 1998. "Nuclear Waste Glass Product Consistency Test (PCT) Method." In: Glass Technology Manual L13.1, Technical Reference, GTOP-3-025, Rev. 3, Aiken, South Carolina.

Savannah River Technology Center (SRTC). 1999. "Determination of Glass Viscosity." In: Glass Technology Manual L13.1, Technical Reference, GTOP-3-111, Rev. 0, Aiken, South Carolina.

Schumacher, R. F. and D. K. Peeler. 1998. Establishment of Harrop, High Temperature Viscometer (U), WSRC-RP-98-00737, Rev. 0, Westinghouse Savannah River Company, Aiken, South Carolina.

Soper, P. D., D. D. Walker, M. J. Plodinec, G. J. Roberts and L. F. Lightner. 1983. "Optimization of Glass Composition for the Vitrification of Nuclear Waste at the Savannah River Plant," Ceram. Bull. Volume 62, No. 9, pp. 1013-1018.

Stone, M. E., and D. P. Lambert. 2000. DWPF Macrobatch 2 Melt Rate Tests, WSRC-TR-200000395, Westinghouse Savannah River Company, Aiken, South Carolina.

Stone, M. E. and J. E. Josephs. 2001. Melt Rate Improvements for DWPF MB3: Melt Rate Furnace Testing, WSRC-TR-2001-00146, Rev. 0, Westinghouse Savannah River Company, Aiken, South Carolina.

Stone, M. E. and D. P. Lambert. 2001. Melt Rate Improvement for MB3: Feed Preparation, WSRC-TR-2001-00126, Rev. 0, Westinghouse Savannah River Company, Aiken, South Carolina.

Tomozawa, M. 1972. "Liquid Phase Separation and Crystal Nucleation in $\mathrm{Li}_{2} \mathrm{O}-\mathrm{SiO}_{2}$ Glasses," Phys. Chem. Glasses, 13, 161-166.

Westinghouse Savannah River Company (WSRC). 2000. High Level Waste System Plan, Rev. 11, HLW-2000-00019, Aiken, South Carolina. 
Immobilization Technology Section

Savannah River Technology Center

Rev. 0

Westinghouse Savannah River Company

Appendix A

Frit and Glass Nomenclature 


\begin{tabular}{|c|c|c|c|}
\hline \multicolumn{2}{|c|}{ Frit } & \multicolumn{2}{|c|}{ Glass } \\
\hline $\begin{array}{c}\text { Previous Reference } \\
\text { Name }\end{array}$ & $\begin{array}{c}\text { New Reference } \\
\text { Name }\end{array}$ & (nominal wash) & (underwashed) \\
\hline $\mathrm{A}$ & Frit 301 & MB3N301-(q or clc) & MB3U301-(q or clc) \\
\hline $\mathrm{B}$ & Frit 302 & MB3N302 & MB3U302 \\
\hline $\mathrm{C}$ & Frit 303 & MB3N303 & MB3U303 \\
\hline $\mathrm{D}$ & Frit 304 & MB3N304 & MB3U304 \\
\hline $\mathrm{E}$ & Frit 305 & MB3N305 & MB3U305 \\
\hline $\mathrm{F}$ & Frit 306 & MB3N306 & MB3U306 \\
\hline $\mathrm{G}$ & Frit 307 & MB3N307 & MB3U307 \\
\hline $\mathrm{H}$ & Frit 308 & MB3N308 & MB3U308 \\
\hline $\mathrm{I}$ & Frit 309 & MB3N309 & MB3U309 \\
\hline $\mathrm{J}$ & Frit 310 & MB3N310 & MB3U310 \\
\hline $\mathrm{K}$ & Frit 311 & MB3N311 & MB3U311 \\
\hline $\mathrm{L}$ & Frit 312 & MB3N312 & MB3U312 \\
\hline $\mathrm{M}$ & Frit 313 & MB3N313 & MB3U313 \\
\hline $\mathrm{N}$ & Frit 314 & MB3N314 & MB3U314 \\
\hline $\mathrm{O}$ & Frit 315 & MB3N315 & MB3U315 \\
\hline $\mathrm{P}$ & Frit 316 & MB3N316 & MB3U316 \\
\hline $\mathrm{Q}$ & Frit 317 & MB3N317 & MB3U317 \\
\hline $\mathrm{R}$ & Frit 318 & MB3N318 & MB3U318 \\
\hline $\mathrm{S}$ & Frit 319 & MB3N319 & MB3U319 \\
\hline $\mathrm{T}$ & Frit 320 & MB3N320 & MB3U320 \\
\hline $\mathrm{U}$ & Frit 321 & MB3N321 & MB3U321 \\
\hline Mimi & Frit 322 & MB3N322 & MB3U322 \\
\hline KMA-2 & Frit 323 & MB3N323 & MB3U323 \\
\hline KMA-2a & Frit 324 & MB3N324 & MB3U324 \\
\hline Bick & Frit 325 & MB3N325 & MB3U325 \\
\hline Bone2 (t adjusted) & Frit 326 & MB3N326 & MB3U326 \\
\hline
\end{tabular}


Immobilization Technology Section

Savannah River Technology Center

Rev. 0

Westinghouse Savannah River Company

\section{Appendix B}

Analytical Plan for Measuring Chemical Compositions (SRT-SCS-2001-00008) 


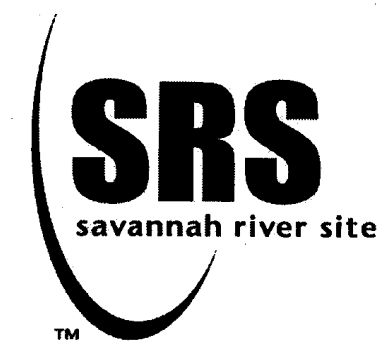

\section{WESTINGHOUSE SAVANNAH RIVER COMPANY INTEROFFICE MEMORANDUM}

SRT-SCS-2001-00008

January 19, 2001

To: D. K. Peeler, 773-43A (wi)

cc: D. F. Bickford, 773-43A (wi)

D. R. Best, 773-41A (wo)

K. G. Brown, 773-43A (wi)

E. M. Frickey, 786-1 A (wo)

D. P. Lambert, 704-1 T (wi)

T. H. Lorier, 773-23A (wi)

S. L. Marra, 704-T (wi)

36

From: T. B. Edwards, 773-42A (5-5148) Statistical Consulting Section
I. R. Reamer, 773̧-A (wi)

E. P. Shine, 773-4A (wi)

M. E. Stone, 704-1T (wi)

R. C. Tuckfield, 773-42A (wi)

D. C. Witt, 704-1T (wi)

R. J. Workman, 773-A (wi)

wi - with sample identifiers

wo - without sample identifiers

es - executive summary only ex Phus

E. P. Shine, Technical Reviewer

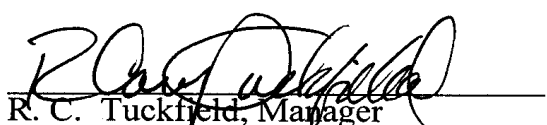

R.C. Tuckfjetd, Mawager

Statistical Consulting Section
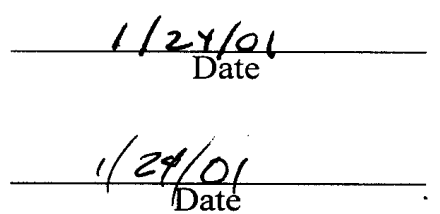

An Analytical Plan for the SRTC Mobile Laboratory to Follow in Measuring the Chemical Compositions of Glasses Supporting the MeltRate Study (U) 


\section{EXECUTIVE SUMMARY}

A task technical and quality assurance plan has been prepared to direct activities associated with SRTC glass studies investigating melt-rate. One aspect of the melt-rate study is the selection of a frit composition to improve melt-rate. Thirteen frit formulations have been selected for study, and glasses were fabricated using each of these frits and a waste simulant representing normal and underwashed, Tank 8 and 40 sludge. This resulted in 26 glasses, and the chemical compositions of the 26 glasses are to be determined by the SRTC Mobile Laboratory (SRTC-ML). This memorandum provides an analytical plan for the SRTC-ML to follow in measuring the chemical compositions of the 26 study glasses. 


\section{INTRODUCTION}

A task technical and quality assurance (TT\&QA) plan [1] has been prepared to direct activities associated with increasing the melting rate at the Defense Waste Processing Facility (DWPF). One aspect of the melt-rate study is the selection of a frit composition to improve melt-rate. Thirteen frit formulations have been selected for study, and glasses were fabricated using each of these frits and a waste simulant representing normal and underwashed, Tank 8 and 40 sludge, as defined by Elder [2]. This resulted in 26 glasses, and the chemical compositions of the 26 glasses are to be determined by the SRTC Mobile Laboratory (SRTC-ML). This memorandum provides an analytical plan for the SRTC-ML to follow in measuring the chemical compositions of the 26 study glasses.

\section{DISCUSSION}

Table 1 provides the naming conventions for the 26 glass samples that are to be used by the SRTC-ML in conducting the compositional analyses and in reporting the measurements.17

\section{Table 1: Unique Sample ID's for the 17 Glasses}

\begin{tabular}{|c|c|c|c|}
\hline Original ID & Lab ID & Original ID & Lab ID \\
\hline Frit $165-\mathrm{N}$ & mr11 & Frit $165-U$ & mr16 \\
\hline Frit $200-\mathrm{N}$ & $\mathrm{mr} 21$ & Frit $200-U$ & $\operatorname{mr} 18$ \\
\hline Frit "C" - N & $\operatorname{mr} 10$ & Frit "C" - U & $\mathrm{mr} 05$ \\
\hline Frit "D" - N & $\operatorname{mr} 19$ & Frit "D" - U & mr20 \\
\hline Frit "G" - N & mr24 & Frit "G" - U & $\operatorname{mr} 14$ \\
\hline Frit "M" - N & mr07 & Frit "M" - U & mr02 \\
\hline Frit "N" - N & $\operatorname{mr} 15$ & Frit "N" - U & mr01 \\
\hline Frit "O" - N & mr06 & Frit "O" - U & mr08 \\
\hline Frit “T” - N & $\mathrm{mr} 25$ & Frit “T” - U & $\operatorname{mr} 17$ \\
\hline Frit "Mimi" - N & mr09 & Frit "Mimi" - U & $\mathrm{mr} 22$ \\
\hline Frit KMA-2-N & $\mathrm{mr} 26$ & Frit KMA-2-U & mr23 \\
\hline Frit KMA-2A-N & $\mathrm{mr} 03$ & Frit KMA-2A-U & $\operatorname{mr} 13$ \\
\hline Frit "Bone2"-N & mr04 & Frit "Bone2"-U & $\operatorname{mr} 12$ \\
\hline
\end{tabular}

\section{PREPARATION OF THE SAMPLES}

The analytical procedures used by the SRTC-ML to determine cation concentrations for a glass sample include steps for sample preparation and for calibration of the Inductively Coupled Plasma (ICP) - Emission Spectrometer. These procedural steps are of primary concern in the development of this analytical plan.

The primary dissolution methods that are to be used by the SRTC-ML to complete this compositional study are lithium metaborate (LM) and peroxide fusion (pf). A third dissolution method (microwave fusion, $\mathrm{mf}$ ) is to be used if necessary to assure complete sample dissolution. All three dissolution methods are considered in this analytical plan.

The cation concentrations are to be measured (as weight percents) for the submitted samples prepared using one or more of the dissolution methods for the following elements: aluminum ( $\mathrm{Al}$ ), boron $(\mathrm{B})$, calcium $(\mathrm{Ca})$, chromium $(\mathrm{Cr})$, iron $(\mathrm{Fe})$, lithium $(\mathrm{Li})$, magnesium $(\mathrm{Mg})$, manganese $(\mathrm{Mn})$, sodium $(\mathrm{Na})$, nickel $(\mathrm{Ni})$, silicon $(\mathrm{Si})$, uranium $(\mathrm{U})$, and zirconium $(\mathrm{Zr})$. 
Each of the 26 samples submitted to the SRTC-ML is to be prepared twice by each of the dissolution methods utilized, and the prepared samples are to be read twice by Inductively Coupled Plasma - Emission Spectroscopy, with the instrument being calibrated before each of these two readings (for each of the prepared samples). This will lead to 4 measurements for each cation of interest for each of the 26 samples submitted to the lab. Table 2 provides unique identifiers for the 52 preparations for each dissolution method and a random sequencing scheme for conducting the dissolutions.

In Table 2, the sample identifier has been modified with a suffix consisting of a two-letter indicator for the preparation method (LM for lithium metaborate, pf for peroxide fusion, and $\mathrm{mw}$ for microwave) and a 1-digit indicator for preparation number.

Table 2: Preparation Blocks

\begin{tabular}{|c|c|c|c|c|c|}
\hline $\begin{array}{c}\text { Lithium } \\
\text { Metaborate } \\
\text { Block 1 }\end{array}$ & $\begin{array}{c}\text { Lithium } \\
\text { Metaborate } \\
\text { Block } 2\end{array}$ & $\begin{array}{c}\text { Peroxide } \\
\text { Fusion } \\
\text { Block 1 }\end{array}$ & $\begin{array}{c}\text { Peroxide } \\
\text { Fusion } \\
\text { Block } 2 \\
\end{array}$ & $\begin{array}{c}\text { Microwave } \\
\text { Fusion } \\
\text { (if necessary) } \\
\text { Block 1 }\end{array}$ & $\begin{array}{c}\text { Microwave } \\
\text { Fusion } \\
\text { (if necessary) } \\
\text { Block } 2\end{array}$ \\
\hline mr02LM1 & mr07LM1 & mr02pf1 & mr01pf1 & mr17mw1 & mr10mw1 \\
\hline mr26LM1 & mr05LM1 & mr21pf1 & mr04pf1 & $\mathrm{mr} 25 \mathrm{mw} 1$ & mr05mw1 \\
\hline mr25LM1 & mr07LM2 & mr03pf1 & $\mathrm{mr01pf2}$ & $\mathrm{mr} 17 \mathrm{mw} 2$ & mr19mw1 \\
\hline mr10LM1 & mr16LM1 & mr17pf1 & mr08pf1 & $\mathrm{mr} 25 \mathrm{mw} 2$ & $\mathrm{mr} 24 \mathrm{mw} 1$ \\
\hline mr08LM1 & mr05LM2 & $\operatorname{mr} 17 \mathrm{pf} 2$ & mr08pf2 & mr11mw1 & mr14mw1 \\
\hline mr10LM2 & mr09LM1 & $\mathrm{mr02pf2}$ & $\mathrm{mr04pf2}$ & mr07mw1 & mr24mw2 \\
\hline mr02LM2 & mr16LM2 & mr03pf2 & mr11pf1 & $\mathrm{mr} 12 \mathrm{mw} 1$ & $\mathrm{mr} 05 \mathrm{mw} 2$ \\
\hline mr24LM1 & mr23LM1 & $\mathrm{mr} 21 \mathrm{pf} 2$ & mr07pf1 & $\mathrm{mr} 21 \mathrm{mw} 1$ & mr19mw2 \\
\hline mr04LM1 & mr01LM1 & mr15pf1 & mr20pf1 & mr07mw2 & mr16mw1 \\
\hline mr25LM2 & mr09LM2 & $\operatorname{mr} 15 \mathrm{pf} 2$ & mr22pf1 & $\mathrm{mr} 02 \mathrm{mw} 1$ & $\mathrm{mr} 10 \mathrm{mw} 2$ \\
\hline mr08LM2 & mr17LM1 & mr18pf1 & $\mathrm{mr} 20 \mathrm{pf} 2$ & $\operatorname{mr} 12 \mathrm{mw} 2$ & $\mathrm{mr} 26 \mathrm{mw} 1$ \\
\hline mr15LM1 & mr01LM2 & mr26pf1 & $\mathrm{mr} 07 \mathrm{pf} 2$ & $\mathrm{mr} 21 \mathrm{mw} 2$ & mr03mw1 \\
\hline mr13LM1 & mr03LM1 & mr05pf1 & $\operatorname{mr} 12 \mathrm{pf} 1$ & mr02mw2 & $\operatorname{mr} 14 \mathrm{mw} 2$ \\
\hline mr24LM2 & mr14LM1 & $\operatorname{mr} 18 \mathrm{pf} 2$ & mr09pf1 & mr22mw1 & mr18mw1 \\
\hline mr13LM2 & mr14LM2 & mr23pf1 & mr13pf1 & $\mathrm{mr} 15 \mathrm{mw} 1$ & mr09mw1 \\
\hline mr18LM1 & mr12LM1 & $\mathrm{mr} 25 \mathrm{pf} 1$ & $\operatorname{mr} 11 \mathrm{pf} 2$ & $\mathrm{mr} 20 \mathrm{mw} 1$ & $\mathrm{mr} 26 \mathrm{mw} 2$ \\
\hline mr26LM2 & mr19LM1 & $\mathrm{mr} 25 \mathrm{pf} 2$ & mr10pf1 & $\operatorname{mr} 15 \mathrm{mw} 2$ & mr04mw1 \\
\hline mr11LM1 & mr17LM2 & mr16pf1 & $\mathrm{mr} 13 \mathrm{pf} 2$ & $\mathrm{mr} 22 \mathrm{mw} 2$ & $\mathrm{mr} 18 \mathrm{mw} 2$ \\
\hline mr20LM1 & mr03LM2 & $\mathrm{mr} 24 \mathrm{pf} 1$ & mr19pf1 & $\mathrm{mr} 11 \mathrm{mw} 2$ & $\mathrm{mr} 13 \mathrm{mw} 1$ \\
\hline mr04LM2 & mr21LM1 & mr06pf1 & mr14pf1 & $\mathrm{mr} 20 \mathrm{mw} 2$ & $\mathrm{mr} 16 \mathrm{mw} 2$ \\
\hline mr18LM2 & mr19LM2 & $\mathrm{mr} 23 \mathrm{pf} 2$ & $\mathrm{mr} 22 \mathrm{pf} 2$ & mr08mw1 & $\mathrm{mr} 23 \mathrm{mw} 1$ \\
\hline mr22LM1 & mr12LM2 & $\mathrm{mr} 26 \mathrm{pf} 2$ & $\operatorname{mr} 12 \mathrm{pf} 2$ & $\mathrm{mr} 01 \mathrm{mw} 1$ & $\mathrm{mr} 09 \mathrm{mw} 2$ \\
\hline mr15LM2 & mr06LM1 & mr05pf2 & mr09pf2 & mr06mw1 & $\mathrm{mr} 04 \mathrm{mw} 2$ \\
\hline mr20LM2 & $\mathrm{mr} 21 \mathrm{LM} 2$ & mr16pf2 & $\mathrm{mr} 10 \mathrm{pf} 2$ & mr06mw2 & $\mathrm{mr} 03 \mathrm{mw} 2$ \\
\hline mr22LM2 & mr06LM2 & $\mathrm{mr} 24 \mathrm{pf} 2$ & $\operatorname{mr} 19 \mathrm{pf} 2$ & $\mathrm{mr} 08 \mathrm{mw} 2$ & $\mathrm{mr} 13 \mathrm{mw} 2$ \\
\hline mr11LM2 & mr23LM2 & mr06pf2 & $\mathrm{mr} 14 \mathrm{pf} 2$ & $\mathrm{mr} 01 \mathrm{mw} 2$ & $\mathrm{mr} 23 \mathrm{mw} 2$ \\
\hline
\end{tabular}

\section{MEASUREMENT OF THE SAMPLES WITH THE ICP}

The samples prepared by each of the dissolution methods employed are to be analyzed using ICP instrumentation calibrated for the particular preparation method. After the initial set of cation concentration measurements have been completed for a set of samples, the ICP instrumentation is to be recalibrated and a second set of concentration measurements for the appropriate cations determined.

Two additional glasses are included in this analytical plan to provide an opportunity for checking the performance of the ICP instrumentation over the course of these analyses and for possible bias-correction of the measurements of the other glasses. One of these glasses is the standard, Batch 1, whose composition is provided in Table 3. 
Table 3: Composition of Batch 1 in Weight Percent (wt\%)

\begin{tabular}{|cc|cc|}
\hline Oxide & Wt\% & Oxide & Wt\% \\
$\mathrm{Al}_{2} \mathrm{O}_{3}$ & 4.877 & $\mathrm{MgO}$ & 1.419 \\
$\mathrm{~B}_{2} \mathrm{O}_{3}$ & 7.777 & $\mathrm{MnO}$ & 1.726 \\
$\mathrm{BaO}$ & 0.151 & $\mathrm{Na}_{2} \mathrm{O}$ & 9.003 \\
$\mathrm{CaO}$ & 1.220 & $\mathrm{Nd}_{2} \mathrm{O}_{3}$ & 0.147 \\
$\mathrm{Cr} \mathrm{O}_{3}$ & 0.107 & $\mathrm{NiO}$ & 0.751 \\
$\mathrm{Cr}_{2} \mathrm{O}$ & 0.060 & $\mathrm{RuO}_{2}$ & 0.0214 \\
$\mathrm{CuO}$ & 0.399 & $\mathrm{SiO}_{2}$ & 50.22 \\
$\mathrm{Fe}_{2} \mathrm{O}_{3}$ & 12.839 & $\mathrm{TiO}_{2}$ & 0.677 \\
$\mathrm{~K}_{2} \mathrm{O}$ & 3.327 & $\mathrm{ZrO}_{2}$ & 0.098 \\
$\mathrm{Li}_{2} \mathrm{O}$ & 4.429 & & \\
\hline
\end{tabular}

The second glass that will be used as a standard for these measurements is a uranium glass that is to be provided to the SRTC-ML along with other glass samples.

A randomized plan for measuring cation concentrations in the prepared samples by each dissolution method is provided in Tables 4-6. In these tables, the sample identifiers have been modified by the addition of a one-digit suffix to indicate whether the measurement is to be made during the first or second ICP calibration block for that sample.

Samples of the standards, Batch 1 and the uranium-bearing glasses, which are to be prepared using the appropriate dissolution method, have been added to Tables 4-6. The identifiers for the Batch 1 standard samples begin with the 3-letter designation "std" followed by the 2-letter dissolution indicator, then the 2digit ICP block number, and finally, a number 1 through 3 for the three replicates of this glass per block. The identifiers for the uranium standard samples begin with the 4-letter designation "ustd" followed by the 2-letter dissolution indicator, then the 2-digit ICP block number, and finally, a number 1 through 2 for the duplicate measurements per block of this glass.

Table 4: ICP Blocks for Samples Prepared Using Lithium Metaborate (LM)

\begin{tabular}{|cccccccc|}
\hline 1-1 & 1-2 & 2-1 & 2-2 & 3-1 & 3-2 & 4-1 & 4-2 \\
stdLM111 & stdLM121 & stdLM211 & stdLM221 & stdLM311 & stdLM321 & stdLM411 & stdLM421 \\
mr24LM21 & mr09LM22 & mr23LM21 & mr2LM22 & mr11LM21 & mr03LM22 & mr13LM11 & mr10LM12 \\
mr24LM11 & mr17LM22 & mr25LM11 & mr01LM12 & mr19LM21 & mr04LM12 & mr12LM21 & mr26LM12 \\
ustdLM11 & ustdLM121 & ustdLM211 & ustdLM221 & ustdLM311 & ustdLM321 & ustdLM411 & ustdLM421 \\
mr17LM11 & mr17LM12 & mr14LM11 & mr14LM12 & mr03LM11 & mr11LM22 & mr06LM21 & mr22LM12 \\
mr05LM11 & mr24LM12 & mr01LM11 & mr25LM22 & mr03LM21 & mr19LM22 & mr22LM21 & mr06LM22 \\
mr18LM11 & mr09LM12 & mr01LM21 & mr01LM22 & mr11LM11 & mr11LM12 & mr13LM21 & mr12LM12 \\
stdLM112 & stdLM122 & stdLM212 & stdLM222 & stdLM312 & stdLM322 & stdLM412 & stdLM422 \\
mr20LM11 & mr20LM12 & mr21LM11 & mr23LM22 & mr16LM11 & mr02LM12 & mr26LM11 & mr22LM22 \\
mr15LM11 & mr20LM22 & mr07LM21 & mr21LM12 & mr02LM11 & mr16LM12 & mr10LM11 & mr26LM22 \\
mr20LM21 & mr24LM22 & mr25LM21 & mr07LM22 & mr04LM11 & mr02LM22 & mr22LM11 & mr06LM12 \\
mr09LM11 & mr05LM22 & mr14LM21 & mr25LM12 & mr16LM21 & mr08LM12 & mr10LM21 & mr13LM12 \\
ustdLM112 & ustdLM122 & ustdLM212 & ustdLM222 & ustdLM312 & ustdLM322 & ustdLM412 & ustdLM422 \\
mr18LM21 & mr15LM12 & mr21LM21 & mr07LM12 & mr02LM21 & mr04LM22 & mr12LM11 & mr13LM22 \\
mr17LM21 & mr15LM22 & mr07LM11 & mr23LM12 & mr08LM11 & mr08LM22 & mr06LM11 & mr10LM22 \\
mr15LM21 & mr05LM12 & mr23LM11 & mr14LM22 & mr08LM21 & mr19LM12 & mr26LM21 & mr12LM22 \\
mr05LM21 & mr18LM12 & stdLM213 & stdLM223 & mr19LM11 & mr16LM22 & stdLM413 & stdLM423 \\
mr09LM21 & mr18LM22 & & & mr04LM21 & mr03LM12 & & \\
stdLM113 & stdLM123 & & & stdLM313 & stdLM323 & & \\
\hline
\end{tabular}


Table 5: ICP Blocks for Samples Prepared Using Peroxide Fusion (pf)

\begin{tabular}{|cccccccc|}
\hline 1-1 & $1-2$ & $2-1$ & $2-2$ & $3-1$ & $3-2$ & $4-1$ & $4-2$ \\
stdpf111 & stdpf121 & stdp211 & stdpf221 & stdpf311 & stdpf321 & stdpf411 & stdpf21 \\
mr12pf21 & mr09pf22 & mr16pf11 & mr11pf22 & mr04pf11 & mr02pf12 & mr20pf21 & mr01pf22 \\
mr15pf11 & mr12pf22 & mr21pf21 & mr11pf12 & mr10pf11 & mr02pf22 & mr08pf21 & mr18pf12 \\
ustdpf111 & ustdpf121 & ustdpf211 & ustdpf221 & ustdpf311 & ustdpf321 & ustdpf411 & ustdpf421 \\
mr15pf21 & mr12pf12 & mr16pf21 & mr26pf22 & mr10pf21 & mr13pf22 & mr18pf21 & mr19pf12 \\
mr09pf21 & mr03pf12 & mr26pf11 & mr26pf12 & mr23pf11 & mr04pf22 & mr19pf11 & mr20pf22 \\
mr25pf11 & mr03pf22 & mr11pf21 & mr16pf22 & mr13pf21 & mr10pf12 & mr01pf11 & mr24pf22 \\
stdpf112 & stdpf122 & stdpf212 & stdpf222 & stdpf312 & stdpf322 & stdpf412 & stdpf422 \\
mr25pf21 & mr15pf12 & mr26pf21 & mr14pf12 & mr17pf11 & mr10pf22 & mr24pf11 & mr08pf12 \\
mr03pf11 & mr22pf22 & mr14pf21 & mr16pf12 & mr13pf11 & mr17pf22 & mr24pf21 & mr20pf12 \\
mr12pf11 & mr25pf12 & mr07pf11 & mr14pf22 & mr05pf11 & mr23pf12 & mr20pf11 & mr18pf22 \\
mr22pf21 & mr06pf12 & mr07pf21 & mr07pf22 & mr05pf21 & mr05pf22 & mr01pf21 & mr19pf22 \\
ustdpf112 & ustdpf122 & ustdpf211 & ustdpf222 & ustdpf312 & ustdpf322 & ustdpf412 & ustdpf422 \\
mr06pf21 & mr06pf22 & mr21pf11 & mr21pf22 & mr02pf11 & mr05pf12 & mr18pf11 & mr24pf12 \\
mr22pf11 & mr25pf22 & mr14pf11 & mr21pf12 & mr23pf21 & mr17pf12 & mr08pf11 & mr01pf12 \\
mr06pf11 & mr09pf12 & mr11pf11 & mr07pf12 & mr02pf21 & mr04pf12 & mr19pf21 & mr08pf22 \\
mr09pf11 & mr15pf22 & stdpf213 & stdpf223 & mr17pf21 & mr13pf12 & stdpf413 & stdpf423 \\
mr03pf21 & mr22pf12 & & & mr04pf21 & mr23pf22 & & \\
stdpf113 & stdpf123 & & & stdpf313 & stdpf323 & & \\
\hline
\end{tabular}

Table 6: ICP Blocks for Samples Prepared Using Microwave Fusion (mf)

\begin{tabular}{|c|c|c|c|c|c|c|c|}
\hline 1-1 & $1-2$ & $2-1$ & $2-2$ & 3-1 & $3-2$ & 4-1 & 4-2 \\
\hline stdmf111 & stdmf 121 & stdmf 211 & stdmf 221 & stdmf311 & stdmf 321 & stdmf411 & stdmf421 \\
\hline $\mathrm{mr} 03 \mathrm{mf} 21$ & $\mathrm{mr} 03 \mathrm{mf} 22$ & $\mathrm{mr} 14 \mathrm{mf} 21$ & $\mathrm{mr} 22 \mathrm{mf} 22$ & $\mathrm{mr} 04 \mathrm{mf} 21$ & $\mathrm{mr} 04 \mathrm{mf} 22$ & $\operatorname{mr06mf} 21$ & $\operatorname{mr} 06 \mathrm{mf} 12$ \\
\hline $\operatorname{mr} 19 \mathrm{mf} 11$ & $\mathrm{mr} 21 \mathrm{mf} 12$ & $\operatorname{mr} 18 \mathrm{mf} 21$ & $\operatorname{mr} 11 \mathrm{mf} 12$ & $\operatorname{mr} 10 \mathrm{mf} 11$ & $\mathrm{mr} 25 \mathrm{mf} 12$ & $\mathrm{mr} 23 \mathrm{mf} 21$ & $\mathrm{mr} 20 \mathrm{mf} 12$ \\
\hline ustdmf 111 & ustdmf 121 & ustdmf211 & ustdmf 221 & ustdmf311 & ustdmf 321 & ustdmf411 & ustdmf 421 \\
\hline $\mathrm{mr} 21 \mathrm{mf} 11$ & $\mathrm{mr} 08 \mathrm{mf} 12$ & $\mathrm{mr} 05 \mathrm{mf} 11$ & $\mathrm{mr} 14 \mathrm{mf} 22$ & $\mathrm{mr} 24 \mathrm{mf} 21$ & $\mathrm{mr} 24 \mathrm{mf} 22$ & $\mathrm{mr07mf11}$ & $\mathrm{mr} 23 \mathrm{mf} 12$ \\
\hline $\mathrm{mr} 21 \mathrm{mf} 21$ & $\mathrm{mr02mf12}$ & $\mathrm{mr} 22 \mathrm{mf} 21$ & $\mathrm{mr} 11 \mathrm{mf} 22$ & mr16mf11 & $\mathrm{mr01mf} 22$ & mr06mf11 & $\operatorname{mr} 13 \mathrm{mf} 12$ \\
\hline mr08mf21 & mr09mf12 & $\mathrm{mr} 22 \mathrm{mf} 11$ & $\operatorname{mr} 14 \mathrm{mf} 12$ & $\operatorname{mr} 17 \mathrm{mf} 21$ & $\mathrm{mr} 04 \mathrm{mf} 12$ & $\mathrm{mr} 20 \mathrm{mf} 21$ & $\mathrm{mr} 07 \mathrm{mf} 12$ \\
\hline stdmf112 & stdmf122 & stdmf 212 & stdmf 222 & stdmf 312 & stdmf 322 & stdmf412 & stdmf 422 \\
\hline $\mathrm{mr} 03 \mathrm{mf} 11$ & $\mathrm{mr} 08 \mathrm{mf} 22$ & $\operatorname{mr} 18 \mathrm{mf} 11$ & $\mathrm{mr} 22 \mathrm{mf} 12$ & $\mathrm{mr} 24 \mathrm{mf} 11$ & $\mathrm{mr} 10 \mathrm{mf} 12$ & $\mathrm{mr} 07 \mathrm{mf} 21$ & $\mathrm{mr} 26 \mathrm{mf} 12$ \\
\hline $\mathrm{mr} 15 \mathrm{mf} 21$ & $\mathrm{mr} 15 \mathrm{mf} 22$ & $\mathrm{mr} 05 \mathrm{mf} 21$ & $\mathrm{mr} 12 \mathrm{mf} 22$ & $\mathrm{mr} 25 \mathrm{mf} 21$ & $\mathrm{mr} 10 \mathrm{mf} 22$ & $\operatorname{mr} 13 \mathrm{mf} 21$ & $\operatorname{mr} 06 \mathrm{mf} 22$ \\
\hline $\mathrm{mr} 19 \mathrm{mf} 21$ & $\mathrm{mr} 19 \mathrm{mf} 22$ & mr11mf 21 & $\mathrm{mr} 05 \mathrm{mf} 12$ & $\mathrm{mr} 01 \mathrm{mf} 21$ & $\operatorname{mr} 16 \mathrm{mf} 12$ & $\mathrm{mr} 23 \mathrm{mf} 11$ & $\mathrm{mr} 20 \mathrm{mf} 22$ \\
\hline mr08mf11 & $\mathrm{mr} 02 \mathrm{mf} 22$ & $\mathrm{mr} 12 \mathrm{mf} 21$ & $\mathrm{mr} 05 \mathrm{mf} 22$ & $\mathrm{mr04mf11}$ & mr01mf12 & $\operatorname{mr} 13 \mathrm{mf} 11$ & $\mathrm{mr} 23 \mathrm{mf} 22$ \\
\hline ustdmf 112 & ustdmf 122 & ustdmf 212 & ustdmf 222 & ustdmf 312 & ustdmf 322 & ustdmf412 & ustdmf 422 \\
\hline $\operatorname{mr} 15 \mathrm{mf} 11$ & $\mathrm{mr} 19 \mathrm{mf} 12$ & mr14mf11 & $\mathrm{mr} 18 \mathrm{mf} 22$ & $\mathrm{mr} 25 \mathrm{mf} 11$ & $\mathrm{mr} 17 \mathrm{mf} 22$ & $\mathrm{mr} 26 \mathrm{mf} 11$ & $\mathrm{mr} 13 \mathrm{mf} 22$ \\
\hline mr09mf11 & $\operatorname{mr} 15 \mathrm{mf} 12$ & $\operatorname{mr} 12 \mathrm{mf} 11$ & $\operatorname{mr} 18 \mathrm{mf} 12$ & $\operatorname{mr} 16 \mathrm{mf} 21$ & $\mathrm{mr} 25 \mathrm{mf} 22$ & $\mathrm{mr} 26 \mathrm{mf} 21$ & $\mathrm{mr} 07 \mathrm{mf} 22$ \\
\hline $\mathrm{mr} 09 \mathrm{mf} 21$ & $\mathrm{mr} 03 \mathrm{mf} 12$ & mr11mf11 & $\mathrm{mr} 12 \mathrm{mf} 12$ & $\mathrm{mr} 10 \mathrm{mf} 21$ & $\operatorname{mr} 16 \mathrm{mf} 22$ & $\mathrm{mr} 20 \mathrm{mf} 11$ & $\mathrm{mr} 26 \mathrm{mf} 22$ \\
\hline $\mathrm{mr} 02 \mathrm{mf} 11$ & mr09mf 22 & stdmf 213 & Stdmf 223 & $\operatorname{mr} 17 \mathrm{mf} 11$ & $\operatorname{mr} 17 \mathrm{mf} 12$ & stdmf413 & stdmf 423 \\
\hline $\mathrm{mr} 02 \mathrm{mf} 21$ & $\mathrm{mr} 21 \mathrm{mf} 22$ & & & $\mathrm{mr} 01 \mathrm{mf} 11$ & $\mathrm{mr} 24 \mathrm{mf} 12$ & & \\
\hline stdmf 113 & stdmf123 & & & stdmf 313 & stdmf 323 & & \\
\hline
\end{tabular}




\section{CONCLUDing COMMENTS}

This memorandum provides an analytical plan for the SRTC-ML to follow in measuring the chemical compositions of 26 glasses that support the melt-rate study. The analytical plan identifies several ICP calibration blocks in Tables 4-6 as well as preparation blocks in Table 2. The sequencing of the activities associated with each of the steps in the analytical procedures has been randomized. The size of each of the blocks is such that it should be completed in a single work shift.

If for some reason the measurements are not conducted in the sequences presented in this memorandum, the actual order used should be recorded along with any explanative comments.

The analytical plan provided in the preceding tables should be modified by the personnel of SRTC-ML to include any calibration check standards and/or other standards that are part of their routine operating procedures.

\section{REFERENCES}

[1] Lambert, D. P., D. K. Peeler, M. E. Stone, and T. H. Lorier, “Task Technical and QA Plan: Alternative Process Options to Improve Melt Rate,” WSRC-RP-2001-00183, January 2001.

[2] Elder, H. H., "Sludge Batch 2 Qualification Strategy and Simulant Composition," HLW-SDT-2000-00128, Rev. 0, May 2000. 
Immobilization Technology Section

Savannah River Technology Center

Rev. 0

Westinghouse Savannah River Company

\section{Appendix C}

Analytical Plan for Measuring PCT Solutions: Nominal MB3 Sludge (SRT-SCS-2001-00009) 


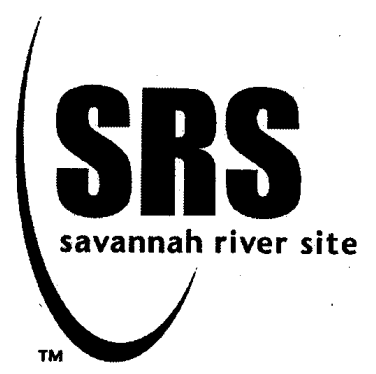

\section{WESTINGHOUSE SAVANNAH RIVER COMPANY INTEROFFICE MEMORANDUM}

SRT-SCS-2001-00009

January 24, 2001

To: D. K. Peeler, 773-43A (wi)
cc: D. F. Bickford, 773-43A (wi)
D. R. Best, 773-41A (wo)
K. G. Brown, 773-43A (wi)
E. M. Frickey, 786-1A (wo)
D. P. Lambert, 704-1T (wi)
T. H. Lorier, 773-23A (wi)
S. L. Marra, 704-T (wi)

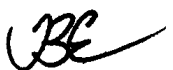

From: T. B. Edwards, 773-42A (5-5148) Statistical Consulting Section
I. R. Reamer, 773-A (wi)

E. P. Shine, 773-4A (wi)

M. E. Stone, 704-1T (wi)

R. C. Tuckfield, 773-42A (wi)

D. C. Witt, 704-1T (wi)

R. J. Workman, 773-A (wi)

wo - without sample identifiers

es - executive summary only

cop Straie $1 / 3 / \% 1$

E. P. Shine, Technical Reviewer

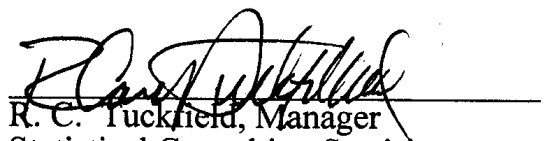

Statistical Consulting Section
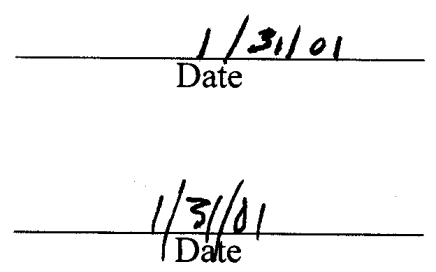

\section{An Analytical Plan for Measuring PCT Solutions of Glasses Representing Nominally Washed Tank 8/40 Sludge (U)}




\section{Executive Summary}

The Immobilization Technology Section currently is exploring improvements in melt-rate for the Defense Waste Processing Facility (DWPF) via changes to the frit composition. Twenty-six glasses were recently batched in support of the study with thirteen of the glasses representing nominally washed Tank $8 / 40$ sludge, while the other 13 represented underwashed sludge. During fabrication, these glasses were cooled by quenching and centerline cooling. The durabilities of both versions of the 26 glasses are to be determined. The Product Consistency Test, or PCT, is used as a measure of glass durability, and its requirements are described in ASTM C1285-97 (Method A). Each PCT results in a leachate solution whose elemental concentrations must be measured to complete the determination of glass durability.

The PCTs are to be conducted in two sets: the glasses representing the nominally washed sludge and the glasses representing the underwashed sludge. This memorandum addresses the PCTs that are being conducted for the thirteen glasses representing nominally washed sludge. (A separate analytical plan is to be issued to cover the PCTs for the thirteen glasses representing underwashed sludge.). Since the glasses were cooled by both quenching and centerline cooling, a total of twenty-six glass samples are to be subjected to the PCT to cover the nominally washed case.

The Savannah River Technology Center-Mobile Laboratory (SRTC-ML) is to measure elemental concentrations of the resulting leachate solutions. This memorandum provides an analytical plan for the SRTC-ML to follow in measuring the compositions of the leachate solutions resulting from the PCT procedures for these glasses. 


\section{INTRODUCTION}

The Immobilization Technology Section currently is exploring improvements in melt-rate for the Defense Waste Processing Facility (DWPF) via changes to the frit composition [1]. Twenty-six glasses were recently batched in support of the study with thirteen of the glasses representing nominally washed Tank $8 / 40$ sludge, while the other 13 represented underwashed sludge. During fabrication, all glasses were cooled both by quenching and by centerline cooling. The durabilities of both versions of the 26 glasses are to be determined. The Product Consistency Test, or PCT, is used as a measure of glass durability, and its requirements are described in ASTM C1285-97 (Method A) [2]. Each PCT results in a leachate solution whose elemental concentrations must be measured to complete the determination of glass durability.

This memorandum addresses the PCTs that are being conducted for the thirteen glasses representing nominally washed sludge. (A separate analytical plan is to be issued to cover the PCTs for the thirteen glasses representing underwashed sludge.). Since the glasses were cooled by both quenching and centerline cooling, there are twenty-six glass samples representing the nominally washed case that are to be subjected to the PCT.

The Savannah River Technology Center Mobile Laboratory (SRTC-ML) is to measure the compositions of the leachate solutions resulting from the PCTs for these glasses, and this memorandum provides an analytical plan for the SRTC-ML to follow in conducting the measurements.

\section{DISCUSSION}

Twenty-six, melt-rate-study, glass samples (those representing the nominally washed Tank 8/40 case) are to be subjected to the PCT. Each of the tests is to be conducted in triplicate. In addition to the test glasses, triplicate PCTs are to be conducted on a sample of the Approved Reference Material (ARM) glass and a sample of the Environmental Assessment (EA) glass. Two reagent blank samples are also to be included in these tests. Thus, a total of 86 samples are required to complete these PCTs.

The leachates from these tests will be diluted by adding $6 \mathrm{~mL}$ of $0.4 \mathrm{HNO}_{3}$ to $4 \mathrm{~mL}$ of the leachate (a 4:10, volume to volume, v:v, dilution) before being submitted to the Mobile Laboratory. The EA leachates will be further diluted $(1: 10, \mathrm{v}: \mathrm{v})$ with deionized water prior to submission to the Mobile Lab in order to prevent problems with the nebulizer.

Table 1 enumerates the study glasses and the standards (EA, ARM, and blanks) and presents identifying codes, ga01 through ga86, for the PCTs. The glass identifiers in Table 1 indicate glasses that were centerline cooled via a "clc" suffix. The naming convention of Table 1 is to be used by the SRTC-ML in analyzing these solutions and reporting the relevant concentration measurements. 18 
Table 1: Solution Identifiers

\begin{tabular}{|c|c|c|c|c|c|}
\hline $\begin{array}{l}\text { Original } \\
\text { Sample }\end{array}$ & $\begin{array}{l}\text { Cooling } \\
\text { Profile }\end{array}$ & $\begin{array}{l}\text { Solution } \\
\text { Identifier }\end{array}$ & $\begin{array}{l}\text { Original } \\
\text { Sample }\end{array}$ & $\begin{array}{l}\text { Cooling } \\
\text { Profile }\end{array}$ & $\begin{array}{l}\text { Solution } \\
\text { Identifier }\end{array}$ \\
\hline Frit "Bone2"-N & quenched & ga75 & Frit "O" - N & quenched & ga48 \\
\hline Frit "Bone2"-N & quenched & ga10 & Frit “O” - N & quenched & ga19 \\
\hline Frit "Bone2"-N & quenched & ga27 & Frit "O" - N & $\mathrm{clc}$ & ga72 \\
\hline Frit "Bone2"-N & clc & ga06 & Frit "O" - N & clc & ga04 \\
\hline Frit "Bone2"-N & clc & ga50 & Frit "O" - N & clc & ga51 \\
\hline Frit "Bone2"-N & clc & ga76 & Frit "T" $-\mathrm{N}$ & quenched & ga34 \\
\hline Frit “C” - N & quenched & ga58 & Frit " $\mathrm{T}$ " - N & quenched & ga61 \\
\hline Frit "C" $-N$ & quenched & ga23 & Frit “T" $-\mathrm{N}$ & quenched & ga52 \\
\hline Frit "C" $-\mathrm{N}$ & quenched & ga69 & Frit "T" - N & $\mathrm{clc}$ & ga08 \\
\hline Frit "C" $-\mathrm{N}$ & clc & ga83 & Frit "T" - N & clc & ga40 \\
\hline Frit "C" $-\mathrm{N}$ & clc & ga22 & Frit "T" - N & clc & ga73 \\
\hline Frit "C" $-\mathrm{N}$ & clc & ga29 & Frit $165-\mathrm{N}$ & quenched & ga11 \\
\hline Frit "D" - N & quenched & ga63 & Frit $165-\mathrm{N}$ & quenched & ga70 \\
\hline Frit "D" - N & quenched & ga17 & Frit $165-\mathrm{N}$ & quenched & ga85 \\
\hline Frit "D" - N & quenched & ga36 & Frit $165-\mathrm{N}$ & $\mathrm{clc}$ & ga57 \\
\hline Frit "D" - N & clc & ga18 & Frit $165-\mathrm{N}$ & clc & ga81 \\
\hline Frit "D" - N & clc & ga59 & Frit $165-\mathrm{N}$ & clc & ga30 \\
\hline Frit "D" - N & clc & ga14 & Frit $200-\mathrm{N}$ & quenched & ga54 \\
\hline Frit “G” - N & quenched & ga42 & Frit $200-\mathrm{N}$ & quenched & ga35 \\
\hline Frit " $G$ " - N & quenched & ga82 & Frit $200-\mathrm{N}$ & quenched & ga68 \\
\hline Frit “ $G$ " - N & quenched & $\mathrm{ga03}$ & Frit $200-\mathrm{N}$ & $\mathrm{clc}$ & ga66 \\
\hline Frit "G" - N & clc & ga49 & Frit $200-\mathrm{N}$ & clc & ga43 \\
\hline Frit “G” - N & clc & ga05 & Frit $200-\mathrm{N}$ & clc & ga44 \\
\hline Frit "G" - N & clc & $\mathrm{ga} 07$ & Frit KMA-2A-N & quenched & ga28 \\
\hline Frit "M" - N & quenched & ga25 & Frit KMA-2A-N & quenched & ga84 \\
\hline Frit "M" - N & quenched & ga15 & Frit KMA-2A-N & quenched & ga12 \\
\hline Frit "M" - N & quenched & ga74 & Frit KMA-2A-N & $\mathrm{clc}$ & ga78 \\
\hline Frit "M" - N & $\mathrm{clc}$ & ga21 & Frit KMA-2A-N & clc & ga67 \\
\hline Frit "M" - N & clc & ga47 & Frit KMA-2A-N & clc & ga60 \\
\hline Frit “M" - N & clc & ga26 & Frit KMA-2-N & quenched & ga64 \\
\hline Frit "Mimi" - N & quenched & ga55 & Frit KMA-2-N & quenched & ga65 \\
\hline Frit "Mimi" - N & quenched & ga39 & Frit KMA-2-N & quenched & ga24 \\
\hline Frit "Mimi" - N & quenched & ga71 & Frit KMA-2-N & clc & ga37 \\
\hline Frit "Mimi" - N & clc & ga16 & Frit KMA-2-N & clc & ga62 \\
\hline Frit "Mimi" - N & clc & ga20 & Frit KMA-2-N & clc & ga45 \\
\hline Frit "Mimi" - N & clc & ga79 & EA & & ga80 \\
\hline Frit “N” - N & quenched & ga13 & EA & & ga53 \\
\hline Frit "N" - N & quenched & ga38 & EA & & ga86 \\
\hline Frit "N" - N & quenched & ga46 & ARM & & ga56 \\
\hline Frit "N" - N & $\mathrm{clc}$ & ga33 & ARM & & ga31 \\
\hline Frit "N" - N & clc & ga77 & ARM & & ga02 \\
\hline Frit "N" - N & clc & ga01 & blank & & ga41 \\
\hline Frit "O" - N & quenched & ga32 & blank & & ga09 \\
\hline
\end{tabular}

\section{AnALYTical PLaN}

The analytical plan for the Mobile Lab is provided in this section. Each of the solution samples submitted to the SRTC-ML is to be analyzed only once for each of the following: boron (B), lithium ( $\mathrm{Li}$ ), sodium $(\mathrm{Na})$, and silicon $(\mathrm{Si})$. These measurements are to be made in parts per million (ppm). The analytical procedure used by the SRTC-ML to determine the relevant concentrations involves an Inductively Coupled Plasma (ICP) - Emission Spectrometer. The PCT solutions (as identified in Table 1) are grouped in six ICP blocks for processing by the Mobile Lab in Table 2. Each block will probably require a different calibration of the ICP. 
Table 2: ICP Calibration Blocks for Leachate Measurements

\begin{tabular}{|c|c|c|c|c|c|}
\hline 1 & $\mathbf{2}$ & $\mathbf{3}$ & $\mathbf{4}$ & $\mathbf{5}$ & $\mathbf{6}$ \\
ga58 & std-b2-1 & std-b3-1 & std-b4-1 & std-b5-1 & std-b6-1 \\
ga18 & ga50 & ga76 & ga66 & ga61 & ga52 \\
ga78 & ga62 & ga86 & ga54 & ga48 & ga03 \\
ga63 & ga67 & ga69 & ga57 & ga38 & ga02 \\
ga41 & ga10 & ga12 & ga11 & ga81 & ga44 \\
ga55 & ga39 & ga27 & ga56 & ga40 & ga01 \\
ga75 & ga17 & ga29 & ga08 & ga05 & ga73 \\
ga64 & ga53 & ga45 & std-b4-2 & std-b5-2 & std-b6-2 \\
std-b1-2 & std-b2-2 & std-b3-2 & ga33 & ga70 & ga51 \\
ga21 & ga22 & ga26 & ga42 & ga31 & ga68 \\
ga83 & ga65 & ga71 & ga72 & ga35 & ga09 \\
ga80 & ga15 & ga60 & ga13 & ga82 & ga19 \\
ga37 & ga20 & ga36 & ga32 & ga04 & ga46 \\
ga06 & ga47 & ga74 & ga34 & ga43 & ga07 \\
ga16 & ga84 & ga14 & std-b4-3 & std-b5-3 & ga85 \\
ga25 & ga23 & ga24 & & & std-b6-3 \\
ga28 & std-b2-3 & std-b3-3 & & & \\
std-b1-3 & & & & & \\
\hline
\end{tabular}

A multi-element solution standard (denoted by "std-bi-j" where i=1, 2, 3, 4, 5, and 6 represents the block number and $j=1,2,3$ represents the position in the block) was added at the beginning, middle, and end of each of the six blocks. This standard may be useful in checking and correcting for bias in the concentration measurements resulting from the ICP calibrations.

\section{CONCLUDING COMMENTS}

In summary, this analytical plan identifies six ICP calibration blocks in Table 2 that are to be used by the SRTC-ML in conducting the boron (B), lithium (Li), sodium (Na), and silicon (Si) concentration measurements for the PCTs that are being conducted for the thirteen melt-rate glasses representing nominally washed Tank 8/40 sludge. The sequencing of the activities associated with each of these steps in the analytical procedures has been randomized. The size of the blocks was selected so that each block could be completed in a single work shift. If for some reason the measurements are not conducted in the sequence presented in this memorandum, the actual order used should be recorded along with any explanative comments.

The analytical plan indicated in the preceding tables should be modified by the personnel of SRTC-ML to include any calibration check standards and/or other standards that are part of the standard operating procedures.

\section{REFERENCES}

[1] Lambert, D. P., D. K. Peeler, M. E. Stone, and T. H. Lorier, "Task Technical and QA Plan: Alternative Process Options to Improve Melt Rate,’ WSRC-RP-2001-00183, January 2001.

[2] ASTM C1285-97, "Standard Test Methods for Determining Chemical Durability of Nuclear Waste Glasses: The Product Consistency Test (PCT)," 1997. 
Immobilization Technology Section

Savannah River Technology Center

Rev. 0

Westinghouse Savannah River Company

\section{Appendix D}

Analytical Plan for Measuring PCT Solutions: Underwashed MB3 Sludge (SRT-SCS-2001-00011) 


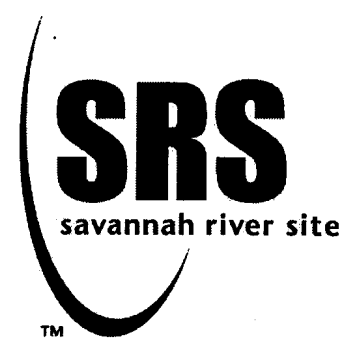

\section{WESTINGHOUSE SAVANNAH RIVER COMPANY INTEROFFICE MEMORANDUM}

SRT-SCS-2001-00011

February 6, 2001

To: D. K. Peeler, 773-43A (wi)

cc: D. F. Bickford, 773-43A (wi)

D. R. Best, 773-41A (wo)

K. G. Brown, 773-43A (wi)

E. M. Frickey, 786-1A (wo)

D. P. Lambert, 704-1 T (wi)

T. H. Lorier, 773-23A (wi)

S. L. Marra, 704-T (wi)

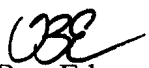

From: T. B. Edwards, 773-42A (5-5148)

Statistical Consulting Section
I. R. Reamer, 773-A (wi)

E. P. Shine, 773-4A (wi)

M. E. Stone, 704-1T (wi)

R. C. Tuckfield, 773-42A (wi)

D. C. Witt, 704-1T (wi)

R. J. Workman, 773-A (wi)
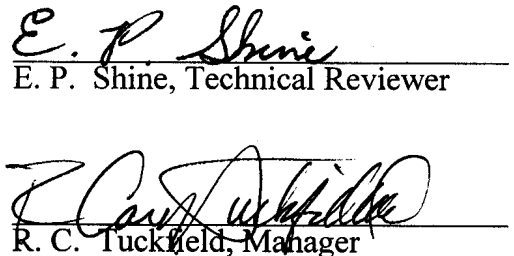

. C. Tuck her, Manager

Statistical Consulting Section wi - with sample identifiers

wo - without sample identifiers

es - executive summary only

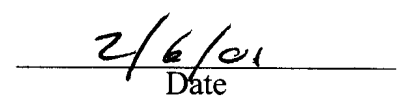

$2 / 6 / 01$

\section{An Analytical Plan for Measuring PCT Solutions of Glasses Representing Under- Washed Tank 8/40 Sludge (U)}




\section{EXECUTIVE SUMMARY}

The Immobilization Technology Section currently is exploring improvements in melt-rate for the Defense Waste Processing Facility (DWPF) via changes to the frit composition. Twenty-six glasses were recently batched in support of the study with thirteen of the glasses representing nominally washed Tank 8/40 sludge, while the other 13 represented underwashed sludge. During fabrication, these glasses were cooled by quenching and centerline cooling. The durabilities of both versions of the 26 glasses are to be determined. The Product Consistency Test, or PCT, is used as a measure of glass durability, and its requirements are described in ASTM C1285-97 (Method A). Each PCT results in a leachate solution whose elemental concentrations must be measured to complete the determination of glass durability.

The PCTs are to be conducted in two sets: the glasses representing the nominally washed sludge and the glasses representing the underwashed sludge. This memorandum addresses the PCTs that are being conducted for the thirteen glasses representing underwashed sludge. (A separate analytical plan was previously issued to cover the PCTs for the thirteen glasses representing nominally washed sludge.) Since the glasses were cooled both by quenching and by centerline cooling, a total of twenty-six glass samples are to be subjected to the PCT to cover the underwashed case.

The Savannah River Technology Center-Mobile Laboratory (SRTC-ML) is to measure elemental concentrations of the resulting leachate solutions. This memorandum provides an analytical plan for the SRTC-ML to follow in measuring the compositions of the leachate solutions resulting from the PCT procedures for these glasses. 


\section{INTRODUCTION}

The Immobilization Technology Section currently is exploring improvements in melt-rate for the Defense Waste Processing Facility (DWPF) via changes to the frit composition [1]. Twenty-six glasses were recently batched in support of the study with thirteen of the glasses representing nominally washed Tank 8/40 sludge, while the other 13 represented underwashed sludge. During fabrication, all glasses were cooled both by quenching and by centerline cooling. The durabilities of both versions of the 26 glasses are to be determined. The Product Consistency Test, or PCT, is used as a measure of glass durability, and its requirements are described in ASTM C1285-97 (Method A) [2]. Each PCT results in a leachate solution whose elemental concentrations must be measured to complete the determination of glass durability.

This memorandum addresses the PCTs that are being conducted for the thirteen glasses representing underwashed sludge. (A separate analytical plan was issued to cover the PCTs for the thirteen glasses representing nominally washed sludge.) Since the glasses were cooled both by quenching and by centerline cooling, there are twenty-six glass samples representing the underwashed case that are to be subjected to the PCT.

The Savannah River Technology Center Mobile Laboratory (SRTC-ML) is to measure the compositions of the leachate solutions resulting from the PCTs for these glasses, and this memorandum provides an analytical plan for the SRTC-ML to follow in conducting the measurements.

\section{DISCUSSION}

Twenty-six, melt-rate-study, glass samples (those representing the underwashed Tank 8/40 case) are to be subjected to the PCT. Each of the tests is to be conducted in triplicate. In addition to the test glasses, triplicate PCTs are to be conducted on a sample of the Approved Reference Material (ARM) glass and a sample of the Environmental Assessment (EA) glass. Two reagent blank samples are also to be included in these tests. Thus, a total of 86 samples are required to complete these PCTs.

The leachates from these tests will be diluted by adding $6 \mathrm{~mL}$ of $0.4 \mathrm{HNO}_{3}$ to $4 \mathrm{~mL}$ of the leachate (a $4: 10$, volume to volume, v:v, dilution) before being submitted to the Mobile Laboratory. The EA leachates will be further diluted $(1: 10, \mathrm{v}: \mathrm{v})$ with deionized water prior to submission to the Mobile Lab in order to prevent problems with the nebulizer.

Table 1 enumerates the study glasses and the standards (EA, ARM, and blanks) and presents identifying codes, jp01 through jp86, for the PCTs. The glass identifiers in Table 1 indicate glasses that were centerline cooled via a "clc" suffix. The naming convention of Table 1 is to be used by the SRTC-ML in analyzing these solutions and reporting the relevant concentration measurements. 19 
Table 1: Solution Identifiers

\begin{tabular}{|c|c|c|c|c|c|}
\hline $\begin{array}{l}\text { Original } \\
\text { Sample }\end{array}$ & $\begin{array}{l}\text { Cooling } \\
\text { Profile }\end{array}$ & $\begin{array}{l}\text { Solution } \\
\text { Identifier }\end{array}$ & $\begin{array}{l}\text { Original } \\
\text { Sample }\end{array}$ & $\begin{array}{l}\text { Cooling } \\
\text { Profile }\end{array}$ & $\begin{array}{l}\text { Solution } \\
\text { Identifier }\end{array}$ \\
\hline Frit "Bone2"-U & quenched & jp47 & Frit "O" - U & quenched & jp86 \\
\hline Frit "Bone2"-U & quenched & jp02 & Frit "O" - U & quenched & jp05 \\
\hline Frit "Bone2"-U & quenched & jp09 & Frit "O" - U & $\mathrm{clc}$ & jp44 \\
\hline Frit "Bone2"-U & clc & jp45 & Frit "O" - U & $\mathrm{clc}$ & jp12 \\
\hline Frit "Bone2"-U & clc & jp26 & Frit "O" - U & clc & jp58 \\
\hline Frit “Bone2"-U & clc & jp34 & Frit "T" - U & quenched & jp70 \\
\hline Frit “C”" - U & quenched & jp50 & Frit "T" - U & quenched & jp32 \\
\hline Frit "C" - U & quenched & jp19 & Frit "T" - U & quenched & jp04 \\
\hline Frit "C" - U & quenched & jp59 & Frit “T" - U & clc & jp21 \\
\hline Frit "C" - U & clc & jp78 & Frit "T" - U & clc & jp76 \\
\hline Frit "C'" - U & clc & jp13 & Frit "T" - U & clc & jp53 \\
\hline Frit "C" - U & clc & jp10 & Frit $165-U$ & quenched & jp73 \\
\hline Frit "D" - U & quenched & jp24 & Frit $165-\mathrm{U}$ & quenched & jp55 \\
\hline Frit "D" - U & quenched & jp56 & Frit $165-U$ & quenched & jp65 \\
\hline Frit "D" - U & quenched & jp07 & Frit $165-\mathrm{U}$ & clc & jp79 \\
\hline Frit "D" - U & clc & jp42 & Frit $165-U$ & clc & jp17 \\
\hline Frit "D" - U & clc & jp62 & Frit $165-U$ & clc & jp48 \\
\hline Frit "D" - U & clc & jp16 & Frit $200-U$ & quenched & jp33 \\
\hline Frit “G” - U & quenched & jp60 & Frit $200-\mathrm{U}$ & quenched & jp75 \\
\hline Frit "G" - U & quenched & jp61 & Frit $200-U$ & quenched & jp46 \\
\hline Frit “G” - U & quenched & jp82 & Frit $200-\mathrm{U}$ & clc & jp68 \\
\hline Frit " $G$ " - U & $\mathrm{clc}$ & jp38 & Frit $200-\mathrm{U}$ & clc & jp41 \\
\hline Frit “G” - U & clc & jp51 & Frit $200-U$ & clc & jp15 \\
\hline Frit "G" - U & clc & jp35 & Frit KMA-2A-U & quenched & jp03 \\
\hline Frit "M" - U & quenched & jp14 & Frit KMA-2A-U & quenched & jp77 \\
\hline Frit "M" - U & quenched & jp06 & Frit KMA-2A-U & quenched & jp40 \\
\hline Frit "M" - U & quenched & jp31 & Frit KMA-2A-U & clc & jp80 \\
\hline Frit "M" - U & $\mathrm{clc}$ & jp74 & Frit KMA-2A-U & $\mathrm{clc}$ & jp57 \\
\hline Frit "M" - U & clc & jp84 & Frit KMA-2A-U & clc & jp20 \\
\hline Frit “M” - U & clc & jp28 & Frit KMA-2-U & quenched & jp37 \\
\hline Frit "Mimi" - U & quenched & jp39 & Frit KMA-2-U & quenched & jp49 \\
\hline Frit "Mimi" - U & quenched & jp30 & Frit KMA-2-U & quenched & jp72 \\
\hline Frit "Mimi" - U & quenched & jp81 & Frit KMA-2-U & clc & jp66 \\
\hline Frit "Mimi" - U & clc & jp63 & Frit KMA-2-U & clc & jp29 \\
\hline Frit "Mimi" - U & clc & jp18 & Frit KMA-2-U & clc & jp85 \\
\hline Frit "Mimi" - U & clc & jp25 & EA & & jp83 \\
\hline Frit “N” - U & quenched & jp43 & EA & & jp27 \\
\hline Frit "N" - U & quenched & jp71 & EA & & jp64 \\
\hline Frit "N" - U & quenched & jp08 & ARM & & jp01 \\
\hline Frit "N" - U & $\mathrm{clc}$ & jp69 & ARM & & jp11 \\
\hline Frit "N" - U & clc & jp54 & ARM & & jp67 \\
\hline Frit "N" - U & clc & jp22 & blank & & jp52 \\
\hline Frit "O" - U & quenched & jp23 & blank & & jp36 \\
\hline
\end{tabular}

\section{Analytical Plan}

The analytical plan for the Mobile Lab is provided in this section. Each of the solution samples submitted to the SRTC-ML is to be analyzed only once for each of the following: boron (B), lithium ( $\mathrm{Li}$ ), sodium $(\mathrm{Na})$, and silicon $(\mathrm{Si})$. These measurements are to be made in parts per million (ppm). The analytical procedure used by the SRTC-ML to determine the relevant concentrations involves an Inductively Coupled Plasma (ICP) - Emission Spectrometer. The PCT solutions (as identified in Table 1) are grouped in six ICP blocks for processing by the Mobile Lab in Table 2. Each block will probably require a different calibration of the ICP. 
Table 2: ICP Calibration Blocks for Leachate Measurements

\begin{tabular}{|c|c|c|c|c|c|}
\hline 1 & $\mathbf{2}$ & $\mathbf{3}$ & $\mathbf{4}$ & $\mathbf{5}$ & $\mathbf{6}$ \\
std-b1-1 & std-b2-1 & std-b3-1 & std-b4-1 & std-b5-1 & std-b6-1 \\
jp33 & jp36 & jp46 & jp21 & jp29 & jp05 \\
jp83 & jp56 & jp67 & jp63 & jp02 & jp58 \\
jp79 & jp11 & jp22 & jp03 & jp18 & jp20 \\
jp73 & jp19 & jp31 & jp38 & jp30 & jp09 \\
jp78 & jp75 & jp10 & jp47 & jp77 & jp34 \\
jp14 & jp71 & jp08 & jp45 & jp32 & jp82 \\
jp68 & jp06 & jp59 & jp70 & jp49 & jp85 \\
jp43 & jp62 & jp07 & jp37 & jp86 & jp35 \\
std-b1-2 & std-b2-2 & std-b3-2 & std-b4-2 & std-b5-2 & std-b6-2 \\
jp74 & jp55 & jp15 & jp60 & jp12 & jp04 \\
jp01 & jp84 & jp48 & jp80 & jp57 & jp25 \\
jp42 & jp41 & jp65 & jp39 & jp61 & jp72 \\
jp24 & jp17 & jp16 & jp66 & jp76 & jp53 \\
jp50 & jp54 & jp64 & jp23 & jp51 & jp81 \\
jp52 & jp27 & jp28 & jp44 & jp26 & jp40 \\
jp69 & jp13 & std-b3-3 & std-b4-3 & std-b5-3 & std-b6-3 \\
std-b1-3 & std-b2-3 & & & & \\
\hline
\end{tabular}

A multi-element solution standard (denoted by "std-bi-j" where i=1, 2, 3, 4, 5, and 6 represents the block number and $j=1,2,3$ represents the position in the block) was added at the beginning, middle, and end of each of the six blocks. This standard may be useful in checking and correcting for bias in the concentration measurements resulting from the ICP calibrations.

\section{ConCLUDING COMMENTS}

In summary, this analytical plan identifies six ICP calibration blocks in Table 2 that are to be used by the SRTC-ML in conducting the boron (B), lithium (Li), sodium (Na), and silicon ( $\mathrm{Si}$ ) concentration measurements for the PCTs that are being conducted for the thirteen melt-rate glasses representing underwashed Tank 8/40 sludge. The sequencing of the activities associated with each of these steps in the analytical procedures has been randomized. The size of the blocks was selected so that each block could be completed in a single work shift. If for some reason the measurements are not conducted in the sequence presented in this memorandum, the actual order used should be recorded along with any explanative comments.

The analytical plan indicated in the preceding tables should be modified by the personnel of SRTC-ML to include any calibration check standards and/or other standards that are part of the standard operating procedures.

\section{REFERENCES}

[1] Lambert, D. P., D. K. Peeler, M. E. Stone, and T. H. Lorier, "Task Technical and QA Plan: Alternative Process Options to Improve Melt Rate,” WSRC-RP-2001-00183, January 2001.

[2] ASTM C1285-97, "Standard Test Methods for Determining Chemical Durability of Nuclear Waste Glasses: The Product Consistency Test (PCT)," 1997. 
Immobilization Technology Section

Savannah River Technology Center

Rev. 0

Westinghouse Savannah River Company

\section{Appendix E}

Tables of Chemical Composition Measurements, Tables of PCT Measurements, and Exhibits of Statistical Analyses 
TABLE E1: SRTC-ML Measurements For SAMPLes PrePared USing LithiUM Metaborate (LM) METhOD

\begin{tabular}{|c|c|c|c|c|c|c|c|c|c|c|c|c|c|c|c|}
\hline Block & Sub-Block & Sequence & SRTC-ML ID (LM) & Glass/Frit ID & Glass ID & Shortened Glass ID & $\mathrm{Ca}(\mathrm{v})$ & $\mathrm{Cr}(\mathrm{v})$ & $\mathrm{Fe}(\mathrm{v})$ & $\operatorname{Mg}(v)$ & $\operatorname{Mn}(v)$ & $\mathrm{Na}(\mathrm{v})$ & $\mathrm{Ni}(\mathrm{v})$ & Si(v) & $\mathrm{Zr}(\mathrm{v})$ \\
\hline 1 & 1 & 1 & stdlm111 & Batch 1 & Batch 1 & Batch 1 & 0.910 & 0.071 & 9.02 & 0.863 & 1.31 & 6.62 & 0.561 & 23.2 & 0.091 \\
\hline 1 & 1 & 2 & $\mathrm{mr} 24 \operatorname{lm} 21$ & Frit "G" - N & MB3N307 & N307 & 0.688 & 0.007 & 8.07 & 0.015 & 0.579 & 6.26 & 0.392 & 26.4 & 0.006 \\
\hline 1 & 1 & 3 & $\mathrm{mr} 24 \operatorname{lm} 11$ & Frit "G" - N & MB3N307 & N307 & 0.688 & 0.004 & 8.00 & 0.013 & 0.576 & 6.26 & 0.389 & 26.4 & $<0.002$ \\
\hline 1 & 1 & 4 & ustdlm111 & Ustd & Ustd & Ustd & 0.919 & 0.164 & 9.07 & 0.705 & 2.03 & 8.61 & 0.771 & 21.6 & 0.004 \\
\hline 1 & 1 & 5 & $\mathrm{mr} 17 \mathrm{~lm} 11$ & Frit "T" - U & MB3U320 & U320 & 0.681 & 0.007 & 7.83 & 0.014 & 0.546 & 10.6 & 0.345 & 25.9 & $<0.002$ \\
\hline 1 & 1 & 6 & mr05lm11 & Frit "C" - U & MB3U303 & U303 & 0.678 & 0.014 & 7.95 & 0.019 & 0.556 & 3.96 & 0.380 & 24.3 & $<0.002$ \\
\hline 1 & 1 & 7 & $\mathrm{mr} 18 \operatorname{lm} 11$ & Frit 200 - U & MB3U200 & U200 & 0.665 & 0.010 & 7.83 & 0.913 & 0.542 & 9.83 & 0.376 & 24.2 & $<0.002$ \\
\hline 1 & 1 & 8 & stdlm112 & Batch 1 & Batch 1 & Batch 1 & 0.922 & 0.070 & 9.33 & 0.846 & 1.29 & 6.68 & 0.552 & 24.1 & 0.090 \\
\hline 1 & 1 & 9 & $\mathrm{mr} 20 \operatorname{lm} 11$ & Frit "D" - U & MB3U304 & U304 & 0.695 & 0.040 & 8.36 & 0.015 & 0.563 & 14.0 & 0.396 & 24.6 & $<0.002$ \\
\hline 1 & 1 & 10 & $\mathrm{mr} 15 \operatorname{lm} 11$ & Frit "N" - N & MB3N314 & N314 & 0.684 & 0.006 & 8.46 & 0.015 & 0.573 & 6.90 & 0.392 & 24.2 & 1.03 \\
\hline 1 & 1 & 11 & $\operatorname{mr} 20 \operatorname{lm} 21$ & Frit “D” - U & MB3U304 & U304 & 0.657 & 0.055 & 7.91 & 0.020 & 0.567 & 14.2 & 0.389 & 24.1 & 0.008 \\
\hline 1 & 1 & 12 & mr09lm11 & Frit "Mimi" - N & MB3N322 & N322 & 0.674 & 0.008 & 8.29 & 0.015 & 0.587 & 9.04 & 0.398 & 28.3 & $<0.002$ \\
\hline 1 & 1 & 13 & ustdlm112 & Ustd & Ustd & Ustd & 0.912 & 0.168 & 9.55 & 0.719 & 2.06 & 8.46 & 0.786 & 22.6 & 0.005 \\
\hline 1 & 1 & 14 & $\operatorname{mr} 18 \operatorname{lm} 21$ & Frit $200-\mathrm{U}$ & MB3U200 & U200 & 0.671 & 0.007 & 8.31 & 0.951 & 0.567 & 10.2 & 0.393 & 25.5 & $<0.002$ \\
\hline 1 & 1 & 15 & $\operatorname{mr} 17 \operatorname{lm} 21$ & Frit "T" - U & MB3U320 & U320 & 0.663 & 0.004 & 8.27 & 0.014 & 0.551 & 10.4 & 0.351 & 26.4 & $<0.002$ \\
\hline 1 & 1 & 16 & $\mathrm{mr} 15 \operatorname{lm} 21$ & Frit "N" - N & MB3N314 & $\mathrm{N} 314$ & 0.671 & 0.136 & 8.35 & 0.016 & 0.560 & 6.72 & 0.384 & 22.6 & 0.928 \\
\hline 1 & 1 & 17 & $\mathrm{mr} 05 \operatorname{lm} 21$ & Frit "C" - U & MB3U303 & U303 & 0.713 & 0.009 & 8.28 & 0.025 & 0.577 & 3.93 & 0.389 & 25.1 & 0.010 \\
\hline 1 & 1 & 18 & $\mathrm{mr} 091 \mathrm{~m} 21$ & Frit "Mimi" - N & MB3N322 & N322 & 0.680 & 0.008 & 8.49 & 0.015 & 0.592 & 9.03 & 0.391 & 27.3 & 0.132 \\
\hline 1 & 1 & 19 & stdlm113 & Batch 1 & Batch 1 & Batch 1 & 0.908 & 0.070 & 9.64 & 0.856 & 1.30 & 6.51 & 0.558 & 24.6 & 0.092 \\
\hline 1 & 2 & 1 & stdlm121 & Batch 1 & Batch 1 & Batch 1 & 0.919 & 0.077 & 8.89 & 0.883 & 1.32 & 6.72 & 0.572 & 23.1 & 0.096 \\
\hline 1 & 2 & 2 & $\mathrm{mr} 091 \mathrm{~lm} 22$ & Frit "Mimi" - N & MB3N322 & $\mathrm{N} 322$ & 0.704 & 0.013 & 7.89 & 0.025 & 0.603 & 9.42 & 0.401 & 27.3 & 0.137 \\
\hline 1 & 2 & 3 & $\operatorname{mr} 17 \operatorname{lm} 22$ & Frit "T" - U & MB3U320 & U320 & 0.677 & 0.009 & 7.65 & 0.024 & 0.568 & 10.9 & 0.365 & 25.8 & $<0.002$ \\
\hline 1 & 2 & 4 & ustdlm121 & Ustd & Ustd & Ustd & 0.925 & 0.174 & 9.19 & 0.736 & 2.08 & 8.76 & 0.796 & 21.7 & 0.006 \\
\hline 1 & 2 & 5 & $\mathrm{mr} 17 \mathrm{~lm} 12$ & Frit "T" - U & MB3U320 & U320 & 0.691 & 0.012 & 7.75 & 0.024 & 0.559 & 10.9 & 0.356 & 25.9 & $<0.002$ \\
\hline 1 & 2 & 6 & $\mathrm{mr} 24 \mathrm{~lm} 12$ & Frit "G" - N & MB3N307 & N307 & 0.698 & 0.008 & 8.09 & 0.023 & 0.584 & 6.31 & 0.397 & 26.5 & $<0.002$ \\
\hline 1 & 2 & 7 & mr091m12 & Frit "Mimi" - N & MB3N322 & N322 & 0.688 & 0.013 & 8.00 & 0.023 & 0.587 & 9.46 & 0.399 & 27.7 & $<0.002$ \\
\hline 1 & 2 & 8 & stdlm122 & Batch 1 & Batch 1 & Batch 1 & 0.929 & 0.075 & 8.90 & 0.866 & 1.30 & 7.00 & 0.562 & 23.3 & 0.092 \\
\hline 1 & 2 & 9 & $\mathrm{mr} 201 \mathrm{~lm} 12$ & Frit "D" - U & MB3U304 & U304 & 0.711 & 0.044 & 7.87 & 0.025 & 0.568 & 14.6 & 0.400 & 24.6 & $<0.002$ \\
\hline 1 & 2 & 10 & $\mathrm{mr} 201 \mathrm{~lm} 22$ & Frit "D" - U & MB3U304 & U304 & 0.670 & 0.058 & 7.55 & 0.028 & 0.546 & 14.5 & 0.378 & 23.2 & $<0.002$ \\
\hline 1 & 2 & 11 & $\mathrm{mr} 24 \mathrm{~lm} 22$ & Frit "G" - N & MB3N307 & N307 & 0.704 & 0.011 & 7.98 & 0.023 & 0.577 & 6.42 & 0.393 & 26.3 & 0.005 \\
\hline 1 & 2 & 12 & $\mathrm{mr} 051 \mathrm{~m} 22$ & Frit "C" - U & MB3U303 & U303 & 0.730 & 0.013 & 7.91 & 0.032 & 0.575 & 4.10 & 0.390 & 24.0 & $<0.002$ \\
\hline 1 & 2 & 13 & ustdlm 122 & Ustd & Ustd & Ustd & 0.925 & 0.172 & 9.30 & 0.726 & 2.05 & 8.79 & 0.786 & 22.1 & 0.005 \\
\hline 1 & 2 & 14 & $\operatorname{mr} 15 \operatorname{lm} 12$ & Frit "N" - N & MB3N314 & N314 & 0.692 & 0.010 & 8.31 & 0.023 & 0.572 & 7.16 & 0.394 & 23.7 & 1.03 \\
\hline 1 & 2 & 15 & $\mathrm{mr} 15 \mathrm{~lm} 22$ & Frit "N" - N & MB3N314 & N314 & 0.687 & 0.142 & 7.93 & 0.026 & 0.572 & 7.03 & 0.395 & 21.7 & 0.964 \\
\hline 1 & 2 & 16 & $\mathrm{mr} 051 \mathrm{~m} 12$ & Frit "C" - U & MB3U303 & U303 & 0.686 & 0.018 & 7.94 & 0.030 & 0.578 & 4.08 & 0.395 & 24.2 & 0.011 \\
\hline 1 & 2 & 17 & $\mathrm{mr} 18 \mathrm{~lm} 12$ & Frit 200 - U & MB3U200 & U200 & 0.677 & 0.014 & 7.79 & 0.956 & 0.560 & 10.1 & 0.391 & 24.0 & $<0.002$ \\
\hline 1 & 2 & 18 & $\mathrm{mr} 18 \mathrm{~lm} 22$ & Frit $200-\mathrm{U}$ & MB3U200 & U200 & 0.689 & 0.012 & 8.13 & 0.973 & 0.575 & 10.5 & 0.403 & 25.8 & $<0.002$ \\
\hline 1 & 2 & 19 & stdlm123 & Batch 1 & Batch 1 & Batch 1 & 0.922 & 0.076 & 9.36 & 0.871 & 1.31 & 6.79 & 0.563 & 23.9 & 0.093 \\
\hline 2 & 1 & 1 & stdlm211 & Batch 1 & Batch 1 & Batch 1 & 0.928 & 0.069 & 9.04 & 0.862 & 1.30 & 6.86 & 0.564 & 23.4 & 0.091 \\
\hline 2 & 1 & 2 & $\mathrm{mr} 231 \mathrm{~lm} 21$ & Frit KMA-2-U & MB3U323 & U323 & 0.675 & $<0.003$ & 7.55 & 0.018 & 0.541 & 8.57 & 0.379 & 25.7 & $<0.002$ \\
\hline 2 & 1 & 3 & $\mathrm{mr} 25 \operatorname{lm} 11$ & Frit “T" - N & MB $3 N 320$ & $\mathrm{~N} 320$ & 0.700 & $<0.003$ & 7.87 & 0.017 & 0.571 & 10.6 & 0.380 & 26.0 & $<0.002$ \\
\hline 2 & 1 & 4 & ustdlm211 & Ustd & Ustd & Ustd & 0.928 & 0.163 & 9.29 & 0.709 & 2.02 & 8.69 & 0.775 & 21.9 & $<0.002$ \\
\hline 2 & 1 & 5 & $\mathrm{mr} 14 \mathrm{~lm} 11$ & Frit "G" - U & MB3U307 & U307 & 0.686 & $<0.003$ & 7.80 & 0.018 & 0.544 & 6.61 & 0.346 & 26.0 & 0.070 \\
\hline 2 & 1 & 6 & $\mathrm{mr} 01 \mathrm{~lm} 11$ & Frit "N" - U & MB3U314 & U314 & 0.657 & 0.008 & 7.30 & 0.034 & 0.522 & 7.13 & 0.333 & 24.8 & 0.963 \\
\hline 2 & 1 & 7 & $\mathrm{mr} 011 \mathrm{~lm} 21$ & Frit "N" - U & MB3U314 & U314 & 0.652 & 0.005 & 7.22 & 0.035 & 0.524 & 7.12 & 0.333 & 24.9 & 1.01 \\
\hline 2 & 1 & 8 & stdlm212 & Batch 1 & Batch 1 & Batch 1 & 0.924 & 0.068 & 9.04 & 0.853 & 1.29 & 6.89 & 0.558 & 23.4 & 0.099 \\
\hline 2 & 1 & 9 & $\mathrm{mr} 21 \mathrm{~lm} 11$ & Frit $200-\mathrm{N}$ & MB3N200 & N200 & 0.692 & $<0.003$ & 7.85 & 0.914 & 0.561 & 9.88 & 0.377 & 25.0 & $<0.002$ \\
\hline 2 & 1 & 10 & $\mathrm{mr} 071 \mathrm{~m} 21$ & Frit "M" - N & MB3N313 & N313 & 0.691 & 0.006 & 7.85 & 0.019 & 0.584 & 8.53 & 0.393 & 26.1 & $<0.002$ \\
\hline
\end{tabular}


Table E1: SRTC-ML Measurements for Samples Prepared Using Lithium Metaborate Method (continued)

\begin{tabular}{|c|c|c|c|c|c|c|c|c|c|c|c|c|c|c|c|}
\hline Block & Sub-Block & Sequence & SRTC-ML ID (LM) & Glass/Frit ID & Glass ID & Shortened Glass ID & $\mathrm{Ca}(\mathrm{v})$ & $\mathrm{Cr}(\mathrm{v})$ & $\mathrm{Fe}(\mathrm{v})$ & $\mathrm{Mg}(\mathrm{v})$ & $\mathrm{Mn}(\mathrm{v})$ & $\mathrm{Na}(\mathrm{v})$ & $\mathrm{Ni}(\mathrm{v})$ & $\mathrm{Si}(\mathrm{v})$ & $\mathrm{Zr}(\mathrm{v})$ \\
\hline 2 & 1 & 11 & $\mathrm{mr} 25 \operatorname{lm} 21$ & Frit "T" - N & MB3N320 & N320 & 0.689 & $<0.003$ & 7.93 & 0.020 & 0.594 & 10.6 & 0.396 & 25.6 & $<0.002$ \\
\hline 2 & 1 & 12 & $\operatorname{mr} 14 \operatorname{lm} 21$ & Frit "G" - U & MB3U307 & U307 & 0.657 & $<0.003$ & 7.77 & 0.019 & 0.573 & 6.70 & 0.359 & 25.7 & $<0.002$ \\
\hline 2 & 1 & 13 & ustdlm 212 & Ustd & Ustd & Ustd & 0.917 & 0.172 & 8.85 & 0.750 & 2.14 & 8.70 & 0.813 & 21.3 & $<0.002$ \\
\hline 2 & 1 & 14 & $\mathrm{mr} 21 \mathrm{~lm} 21$ & Frit $200-\mathrm{N}$ & MB3N200 & N200 & 0.670 & $<0.003$ & 7.59 & 0.961 & 0.590 & 9.86 & 0.398 & 24.6 & $<0.002$ \\
\hline 2 & 1 & 15 & mr07lm11 & Frit "M" - N & MB3N313 & $\mathrm{N} 313$ & 0.686 & 0.012 & 7.72 & 0.018 & 0.586 & 8.55 & 0.391 & 25.9 & $<0.002$ \\
\hline 2 & 1 & 16 & $\mathrm{mr} 231 \mathrm{~m} 11$ & Frit KMA-2-U & MB3U323 & U323 & 0.671 & 0.003 & 7.52 & 0.020 & 0.556 & 8.70 & 0.387 & 25.3 & 0.183 \\
\hline 2 & 1 & 17 & stdlm213 & Batch 1 & Batch 1 & Batch 1 & 0.933 & 0.069 & 8.85 & 0.871 & 1.32 & 6.92 & 0.563 & 23.0 & 0.092 \\
\hline 2 & 2 & 1 & stdlm221 & Batch 1 & Batch 1 & Batch 1 & 0.925 & 0.087 & 9.04 & 0.908 & 1.36 & 6.90 & 0.586 & 23.3 & 0.109 \\
\hline 2 & 2 & 2 & $\mathrm{mr} 211 \mathrm{~m} 22$ & Frit $200-\mathrm{N}$ & MB3N200 & N200 & 0.678 & 0.018 & 7.87 & 0.976 & 0.609 & 9.76 & 0.410 & 25.0 & 0.010 \\
\hline 2 & 2 & 3 & $\mathrm{mr} 011 \mathrm{~m} 12$ & Frit "N" - U & MB3U314 & U314 & 0.646 & 0.024 & 7.39 & 0.053 & 0.566 & 7.07 & 0.356 & 24.8 & 1.03 \\
\hline 2 & 2 & 4 & ustdlm221 & Ustd & Ustd & Ustd & 0.904 & 0.188 & 9.24 & 0.765 & 2.15 & 8.64 & 0.818 & 21.6 & 0.027 \\
\hline 2 & 2 & 5 & $\mathrm{mr} 14 \mathrm{~lm} 12$ & Frit "G" - U & MB3U307 & U307 & 0.663 & 0.017 & 7.84 & 0.037 & 0.598 & 6.60 & 0.372 & 25.9 & 0.091 \\
\hline 2 & 2 & 6 & $\mathrm{mr} 25 \operatorname{lm} 22$ & Frit "T" - N & MB3N320 & N320 & 0.679 & 0.018 & 8.15 & 0.036 & 0.622 & 10.4 & 0.410 & 25.7 & 0.011 \\
\hline 2 & 2 & 7 & $\mathrm{mr} 011 \mathrm{~lm} 22$ & Frit "N" - U & MB3U314 & U314 & 0.631 & 0.021 & 7.41 & 0.054 & 0.572 & 7.03 & 0.361 & 25.1 & 1.07 \\
\hline 2 & 2 & 8 & stdlm222 & Batch 1 & Batch 1 & Batch 1 & 0.886 & 0.087 & 9.09 & 0.919 & 1.39 & 6.69 & 0.592 & 23.3 & 0.119 \\
\hline 2 & 2 & 9 & $\mathrm{mr} 23 \operatorname{lm} 22$ & Frit KMA-2-U & MB3U323 & U323 & 0.645 & 0.017 & 7.68 & 0.035 & 0.583 & 8.57 & 0.402 & 25.8 & 0.015 \\
\hline 2 & 2 & 10 & $\mathrm{mr} 21 \mathrm{~lm} 12$ & Frit $200-\mathrm{N}$ & MB3N200 & N200 & 0.664 & 0.018 & 8.01 & 0.971 & 0.606 & 9.74 & 0.401 & 25.1 & 0.010 \\
\hline 2 & 2 & 11 & mr07lm22 & Frit "M" - N & MB3N313 & N313 & 0.665 & 0.021 & 7.95 & 0.035 & 0.616 & 8.70 & 0.409 & 26.2 & 0.009 \\
\hline 2 & 2 & 12 & $\mathrm{mr} 25 \operatorname{lm} 12$ & Frit "T" - N & MB3N320 & N320 & 0.658 & 0.016 & 7.99 & 0.034 & 0.622 & 10.6 & 0.409 & 26.6 & 0.009 \\
\hline 2 & 2 & 13 & ustdlm222 & Ustd & Ustd & Ustd & 0.873 & 0.191 & 9.27 & 0.775 & 2.18 & 8.69 & 0.826 & 21.9 & 0.018 \\
\hline 2 & 2 & 14 & $\operatorname{mr} 07 \mathrm{~lm} 12$ & Frit "M" - N & MB3N313 & N313 & 0.650 & 0.028 & 8.01 & 0.035 & 0.622 & 8.54 & 0.412 & 26.3 & 0.010 \\
\hline 2 & 2 & 15 & $\mathrm{mr} 231 \mathrm{~m} 12$ & Frit KMA-2-U & MB3U323 & U323 & 0.633 & 0.019 & 7.89 & 0.037 & 0.595 & 8.60 & 0.413 & 25.9 & 0.205 \\
\hline 2 & 2 & 16 & $\mathrm{mr} 14 \mathrm{~lm} 22$ & Frit "G" - U & MB3U307 & U307 & 0.626 & 0.019 & 8.07 & 0.036 & 0.600 & 6.88 & 0.373 & 26.1 & 0.011 \\
\hline 2 & 2 & 17 & stdlm223 & Batch 1 & Batch 1 & Batch 1 & 0.872 & 0.088 & 9.30 & 0.925 & 1.39 & 6.89 & 0.597 & 23.8 & 0.109 \\
\hline 3 & 1 & 1 & stdlm311 & Batch 1 & Batch 1 & Batch 1 & 0.913 & 0.083 & 9.07 & 0.890 & 1.33 & 6.76 & 0.574 & 23.4 & 0.090 \\
\hline 3 & 1 & 2 & $\mathrm{mr} 11 \mathrm{~lm} 21$ & Frit $165-\mathrm{N}$ & MB3N165 & N165 & 0.686 & 0.023 & 7.81 & 0.518 & 0.586 & 10.9 & 0.387 & 24.4 & 0.515 \\
\hline 3 & 1 & 3 & mr19lm21 & Frit "D" - N & MB3N304 & N304 & 0.698 & 0.014 & 8.08 & 0.025 & 0.582 & 13.7 & 0.387 & 23.9 & $<0.002$ \\
\hline 3 & 1 & 4 & ustdlm311 & Ustd & Ustd & Ustd & 0.934 & 0.176 & 9.24 & 0.727 & 2.05 & 8.50 & 0.782 & 21.8 & $<0.002$ \\
\hline 3 & 1 & 5 & mr031m11 & Frit KMA-2A-N & MB3N324 & N324 & 0.702 & 0.018 & 7.94 & 0.022 & 0.572 & 6.45 & 0.367 & 25.8 & $<0.002$ \\
\hline 3 & 1 & 6 & mr031m21 & Frit KMA-2A-N & MB3N324 & N324 & 0.694 & 0.017 & 7.99 & 0.022 & 0.576 & 6.46 & 0.372 & 25.8 & $<0.002$ \\
\hline 3 & 1 & 7 & $\mathrm{mr} 11 \mathrm{~lm} 11$ & Frit $165-\mathrm{N}$ & MB3N165 & N165 & 0.699 & 0.014 & 8.14 & 0.500 & 0.572 & 11.0 & 0.376 & 25.0 & 0.505 \\
\hline 3 & 1 & 8 & stdlm312 & Batch 1 & Batch 1 & Batch 1 & 0.930 & 0.080 & 9.16 & 0.856 & 1.30 & 6.70 & 0.558 & 23.5 & 0.093 \\
\hline 3 & 1 & 9 & $\operatorname{mr} 161 \mathrm{~m} 11$ & Frit $165-\mathrm{U}$ & MB3U165 & U165 & 0.702 & 0.018 & 7.76 & 0.509 & 0.553 & 11.2 & 0.362 & 24.3 & 0.508 \\
\hline 3 & 1 & 10 & $\operatorname{mr} 02 \operatorname{lm} 11$ & Frit “M" - U & MB3U313 & U313 & 0.688 & 0.013 & 7.96 & 0.026 & 0.546 & 8.80 & 0.386 & 26.5 & $<0.002$ \\
\hline 3 & 1 & 11 & mr04lm11 & Frit “Bone2"-N & MB3N326 & N326 & 0.710 & 0.021 & 8.04 & 0.483 & 0.574 & 9.73 & 0.378 & 25.6 & $<0.002$ \\
\hline 3 & 1 & 12 & $\mathrm{mr} 161 \mathrm{~m} 21$ & Frit $165-\mathrm{U}$ & MB3U165 & U165 & 0.703 & 0.018 & 7.86 & 0.506 & 0.548 & 11.0 & 0.358 & 24.3 & 0.498 \\
\hline 3 & 1 & 13 & ustdlm 322 & Ustd & Ustd & Ustd & 0.934 & 0.172 & 9.29 & 0.709 & 2.01 & 8.42 & 0.773 & 21.8 & 0.007 \\
\hline 3 & 1 & 14 & mr021m 21 & Frit "M" - U & MB3U313 & U313 & 0.673 & 0.014 & 7.71 & 0.026 & 0.532 & 8.59 & 0.375 & 26.0 & $<0.002$ \\
\hline 3 & 1 & 15 & mr081m11 & Frit "O" - U & MB3U315 & U315 & 0.686 & 0.014 & 8.12 & 0.029 & 0.559 & 4.92 & 0.355 & 25.3 & $<0.002$ \\
\hline 3 & 1 & 16 & mr081m21 & Frit "O" - U & MB3U315 & U315 & 0.655 & 0.014 & 7.55 & 0.023 & 0.509 & 4.45 & 0.360 & 24.9 & $<0.002$ \\
\hline 3 & 1 & 17 & mr19lm11 & Frit “D” - N & MB3N304 & N304 & 0.695 & 0.015 & 8.13 & 0.023 & 0.570 & 13.3 & 0.384 & 24.1 & $<0.002$ \\
\hline 3 & 1 & 18 & mr04lm21 & Frit "Bone2"-N & MB3N326 & N326 & 0.698 & 0.019 & 8.14 & 0.493 & 0.579 & 9.48 & 0.384 & 26.0 & $<0.002$ \\
\hline 3 & 1 & 19 & stdlm313 & Batch 1 & Batch 1 & Batch 1 & 0.931 & 0.079 & 9.19 & 0.854 & 1.29 & 6.56 & 0.558 & 23.5 & 0.086 \\
\hline 3 & 2 & 1 & stdlm321 & Batch 1 & Batch 1 & Batch 1 & 0.917 & 0.071 & 9.03 & 0.868 & 1.31 & 6.85 & 0.562 & 23.2 & 0.090 \\
\hline 3 & 2 & 2 & mr031m22 & Frit KMA-2A-N & MB3N324 & N324 & 0.688 & 0.006 & 7.88 & 0.013 & 0.574 & 6.49 & 0.371 & 25.6 & $<0.002$ \\
\hline 3 & 2 & 3 & $\mathrm{mr} 04 \mathrm{~lm} 12$ & Frit "Bone2"-N & MB3N326 & N326 & 0.697 & 0.011 & 7.96 & 0.493 & 0.582 & 9.86 & 0.382 & 25.4 & $<0.002$ \\
\hline 3 & 2 & 4 & ustdlm 321 & Ustd & Ustd & Ustd & 0.920 & 0.168 & 9.06 & 0.728 & 2.06 & 8.58 & 0.792 & 21.5 & 0.003 \\
\hline 3 & 2 & 5 & $\mathrm{mr} 11 \mathrm{~lm} 22$ & Frit $165-\mathrm{N}$ & MB3N165 & N165 & 0.675 & 0.012 & 7.72 & 0.509 & 0.578 & 10.9 & 0.380 & 24.2 & 0.512 \\
\hline
\end{tabular}


Table E1: SRTC-ML Measurements for Samples Prepared Using Lithium Metaborate Method (continued)

\begin{tabular}{|c|c|c|c|c|c|c|c|c|c|c|c|c|c|c|c|}
\hline Block & Sub-Block & Sequence & SRTC-ML ID (LM) & Glass/Frit ID & Glass ID & Shortened Glass ID & $\mathrm{Ca}(\mathrm{v})$ & $\mathrm{Cr}(\mathrm{v})$ & $\mathrm{Fe}(\mathrm{v})$ & $\mathrm{Mg}(\mathrm{v})$ & $\mathrm{Mn}(\mathrm{v})$ & $\mathrm{Na}(\mathrm{v})$ & $\mathrm{Ni}(\mathrm{v})$ & $\mathrm{Si}(\mathrm{v})$ & $\mathrm{Zr}(\mathrm{v})$ \\
\hline 3 & 2 & 6 & $\operatorname{mr} 19 \operatorname{lm} 22$ & Frit “D”- N & MB3N304 & N304 & 0.690 & 0.003 & 7.89 & 0.014 & 0.576 & 13.6 & 0.384 & 23.5 & $<0.002$ \\
\hline 3 & 2 & 7 & $\mathrm{mr} 11 \operatorname{lm} 12$ & Frit $165-\mathrm{N}$ & MB3N165 & N165 & 0.696 & 0.003 & 7.88 & 0.511 & 0.581 & 11.2 & 0.380 & 24.5 & 0.517 \\
\hline 3 & 2 & 8 & stdlm322 & Batch 1 & Batch 1 & Batch 1 & 0.916 & 0.071 & 8.90 & 0.882 & 1.33 & 6.70 & 0.569 & 23.0 & 0.097 \\
\hline 3 & 2 & 9 & $\operatorname{mr} 02 \operatorname{lm} 12$ & Frit "M" - U & MB3U313 & U313 & 0.668 & $<0.003$ & 7.87 & 0.017 & 0.561 & 8.82 & 0.396 & 26.3 & $<0.002$ \\
\hline 3 & 2 & 10 & $\operatorname{mr} 16 \operatorname{lm} 12$ & Frit $165-\mathrm{U}$ & MB3U165 & U165 & 0.686 & 0.008 & 7.68 & 0.516 & 0.558 & 11.2 & 0.363 & 24.0 & 0.512 \\
\hline 3 & 2 & 11 & mr021m22 & Frit "M" - U & MB3U313 & U313 & 0.682 & 0.004 & 7.66 & 0.016 & 0.542 & 8.69 & 0.381 & 26.0 & $<0.002$ \\
\hline 3 & 2 & 12 & $\mathrm{mr} 08 \operatorname{lm} 12$ & Frit "O" - U & MB3U315 & U315 & 0.681 & $<0.003$ & 7.88 & 0.020 & 0.570 & 5.02 & 0.360 & 24.9 & $<0.002$ \\
\hline 3 & 2 & 13 & ustdlm322 & Ustd & Ustd & Ustd & 0.932 & 0.168 & 7.89 & 0.728 & 2.08 & 9.55 & 0.793 & 20.1 & 0.004 \\
\hline 3 & 2 & 14 & mr04lm22 & Frit "Bone2"-N & MB3N326 & N326 & 0.691 & 0.009 & 6.79 & 0.500 & 0.589 & 10.6 & 0.387 & 25.5 & $<0.002$ \\
\hline 3 & 2 & 15 & mr081m22 & Frit “O” - U & MB3U315 & U315 & 0.641 & 0.003 & 6.37 & 0.014 & 0.524 & 4.54 & 0.366 & 22.3 & $<0.002$ \\
\hline 3 & 2 & 16 & $\operatorname{mr} 19 \operatorname{lm} 12$ & Frit "D" - N & MB3N304 & N304 & 0.685 & 0.005 & 6.96 & 0.014 & 0.588 & 13.6 & 0.394 & 24.3 & $<0.002$ \\
\hline 3 & 2 & 17 & $\mathrm{mr} 16 \mathrm{~lm} 22$ & Frit 165 - U & MB3U165 & U165 & 0.688 & 0.007 & 6.83 & 0.522 & 0.564 & 11.8 & 0.367 & 24.6 & 0.512 \\
\hline 3 & 2 & 18 & $\mathrm{mr} 031 \mathrm{~m} 12$ & Frit KMA-2A-N & MB3N324 & N324 & 0.696 & 0.007 & 7.03 & 0.011 & 0.574 & 6.69 & 0.368 & 25.4 & $<0.002$ \\
\hline 3 & 2 & 19 & stdlm323 & Batch 1 & Batch 1 & Batch 1 & 0.923 & 0.071 & 8.16 & 0.877 & 1.33 & 6.93 & 0.567 & 21.7 & 0.091 \\
\hline 4 & 1 & 1 & stdlm411 & Batch 1 & Batch 1 & Batch 1 & 0.923 & 0.074 & 8.90 & 0.877 & 1.31 & 6.98 & 0.566 & 22.8 & 0.093 \\
\hline 4 & 1 & 2 & $\mathrm{mr} 13 \operatorname{lm} 11$ & Frit KMA-2A-U & MB3U324 & U324 & 0.677 & 0.030 & 7.78 & 0.028 & 0.561 & 6.99 & 0.367 & 24.8 & $<0.002$ \\
\hline 4 & 1 & 3 & $\operatorname{mr} 12 \operatorname{lm} 21$ & Frit "Bone2"-U & MB3U326 & U326 & 0.670 & 0.008 & 8.01 & 0.499 & 0.566 & 10.4 & 0.387 & 25.6 & $<0.002$ \\
\hline 4 & 1 & 4 & ustdlm411 & Ustd & Ustd & Ustd & 0.929 & 0.172 & 9.41 & 0.735 & 2.06 & 8.76 & 0.788 & 21.8 & 0.006 \\
\hline 4 & 1 & 5 & $\operatorname{mr} 061 \mathrm{~lm} 21$ & Frit “O”- N & MB3N315 & N315 & 0.687 & 0.008 & 8.16 & 0.023 & 0.582 & 3.66 & 0.383 & 25.3 & $<0.002$ \\
\hline 4 & 1 & 6 & $\mathrm{mr} 22 \operatorname{lm} 21$ & Frit "Mimi" - U & MB3U322 & U322 & 0.657 & 0.010 & 7.90 & 0.027 & 0.559 & 9.49 & 0.352 & 28.3 & 0.005 \\
\hline 4 & 1 & 7 & $\mathrm{mr} 13 \operatorname{lm} 21$ & Frit KMA-2A-U & MB3U324 & U324 & 0.694 & 0.007 & 8.51 & 0.029 & 0.573 & 7.08 & 0.373 & 26.3 & $<0.002$ \\
\hline 4 & 1 & 8 & stdlm412 & Batch 1 & Batch 1 & Batch 1 & 0.924 & 0.074 & 9.67 & 0.872 & 1.30 & 6.82 & 0.562 & 24.2 & 0.090 \\
\hline 4 & 1 & 9 & $\mathrm{mr} 261 \mathrm{~m} 11$ & Frit KMA-2-N & MB3N323 & N323 & 0.689 & 0.007 & 8.38 & 0.023 & 0.580 & 8.37 & 0.381 & 26.7 & $<0.002$ \\
\hline 4 & 1 & 10 & $\mathrm{mr} 10 \operatorname{lm} 11$ & Frit "C" - N & MB3N303 & N303 & 0.690 & 0.007 & 8.37 & 0.025 & 0.584 & 3.77 & 0.386 & 24.2 & $<0.002$ \\
\hline 4 & 1 & 11 & $\mathrm{mr} 22 \operatorname{lm} 11$ & Frit "Mimi" - U & MB3U322 & U322 & 0.669 & 0.029 & 8.15 & 0.029 & 0.569 & 9.57 & 0.357 & 28.2 & 0.003 \\
\hline 4 & 1 & 12 & $\operatorname{mr} 10 \operatorname{lm} 21$ & Frit "C" - N & MB3N303 & N303 & 0.680 & 0.007 & 8.49 & 0.022 & 0.589 & 3.74 & 0.390 & 24.3 & $<0.002$ \\
\hline 4 & 1 & 13 & ustdlm 412 & Ustd & Ustd & Ustd & 0.917 & 0.171 & 9.76 & 0.738 & 2.07 & 8.78 & 0.792 & 22.4 & 0.007 \\
\hline 4 & 1 & 14 & $\operatorname{mr} 12 \operatorname{lm} 11$ & Frit "Bone2"-U & MB3U326 & U326 & 0.679 & 0.012 & 8.41 & 0.506 & 0.572 & 10.4 & 0.394 & 25.7 & $<0.002$ \\
\hline 4 & 1 & 15 & mr06lm11 & Frit “O” - N & MB3N315 & N315 & 0.681 & 0.007 & 8.67 & 0.023 & 0.592 & 3.68 & 0.389 & 24.9 & $<0.002$ \\
\hline 4 & 1 & 16 & $\mathrm{mr} 261 \mathrm{~m} 21$ & Frit KMA-2-N & MB3N323 & N323 & 0.678 & 0.008 & 8.47 & 0.020 & 0.590 & 8.39 & 0.389 & 27.0 & 0.081 \\
\hline 4 & 1 & 17 & stdlm413 & Batch 1 & Batch 1 & Batch 1 & 0.918 & 0.074 & 9.65 & 0.877 & 1.30 & 6.92 & 0.564 & 24.2 & 0.092 \\
\hline 4 & 2 & 1 & stdlm421 & Batch 1 & Batch 1 & Batch 1 & 0.920 & 0.077 & 9.25 & 0.872 & 1.30 & 6.80 & 0.561 & 23.5 & 0.093 \\
\hline 4 & 2 & 2 & $\mathrm{mr} 10 \operatorname{lm} 12$ & Frit “C” - N & MB3N303 & N303 & 0.692 & 0.008 & 8.10 & 0.028 & 0.573 & 3.70 & 0.378 & 24.2 & $<0.002$ \\
\hline 4 & 2 & 3 & $\mathrm{mr} 261 \mathrm{~m} 12$ & Frit KMA-2-N & MB3N323 & N323 & 0.683 & 0.008 & 8.08 & 0.027 & 0.576 & 8.26 & 0.380 & 26.2 & 0.006 \\
\hline 4 & 2 & 4 & ustdlm 421 & Ustd & Ustd & Ustd & 0.924 & 0.168 & 9.43 & 0.719 & 2.03 & 8.55 & 0.774 & 22.0 & 0.003 \\
\hline 4 & 2 & 5 & $\mathrm{mr} 22 \operatorname{lm} 12$ & Frit "Mimi" - U & MB3U322 & U322 & 0.670 & 0.029 & 7.99 & 0.033 & 0.563 & 9.39 & 0.353 & 28.1 & $<0.002$ \\
\hline 4 & 2 & 6 & mr061m22 & Frit "O" - N & MB3N315 & N315 & 0.680 & 0.009 & 8.21 & 0.028 & 0.578 & 3.55 & 0.382 & 25.4 & $<0.002$ \\
\hline 4 & 2 & 7 & $\mathrm{mr} 12 \operatorname{lm} 12$ & Frit "Bone2"-U & MB3U326 & U326 & 0.679 & 0.013 & 8.14 & 0.497 & 0.561 & 10.2 & 0.386 & 25.7 & $<0.002$ \\
\hline 4 & 2 & 8 & stdlm 422 & Batch 1 & Batch 1 & Batch 1 & 0.927 & 0.074 & 9.49 & 0.866 & 1.29 & 6.80 & 0.560 & 24.0 & 0.090 \\
\hline 4 & 2 & 9 & $\mathrm{mr} 22 \operatorname{lm} 22$ & Frit "Mimi" - U & MB3U322 & U322 & 0.655 & 0.011 & 7.95 & 0.032 & 0.556 & 9.35 & 0.351 & 28.5 & 0.007 \\
\hline 4 & 2 & 10 & $\mathrm{mr} 261 \mathrm{~m} 22$ & Frit KMA-2-N & MB3N323 & $\mathrm{N} 323$ & 0.692 & 0.008 & 8.19 & 0.024 & 0.574 & 8.40 & 0.378 & 26.6 & 0.079 \\
\hline 4 & 2 & 11 & $\operatorname{mr} 061 \mathrm{~m} 12$ & Frit "O" - N & MB3N315 & N315 & 0.692 & 0.008 & 8.28 & 0.028 & 0.578 & 3.65 & 0.381 & 25.8 & $<0.002$ \\
\hline 4 & 2 & 12 & $\mathrm{mr} 13 \operatorname{lm} 12$ & Frit KMA-2A-U & MB3U324 & U324 & 0.681 & 0.029 & 8.26 & 0.032 & 0.554 & 6.84 & 0.362 & 25.9 & $<0.002$ \\
\hline 4 & 2 & 13 & ustdlm 422 & Ustd & Ustd & Ustd & 0.923 & 0.170 & 9.58 & 0.730 & 2.04 & 8.63 & 0.787 & 22.2 & 0.003 \\
\hline 4 & 2 & 14 & $\operatorname{mr} 13 \operatorname{lm} 22$ & Frit KMA-2A-U & MB3U324 & U324 & 0.696 & 0.007 & 8.36 & 0.034 & 0.573 & 7.16 & 0.372 & 26.3 & $<0.002$ \\
\hline 4 & 2 & 15 & $\mathrm{mr} 101 \mathrm{~m} 22$ & Frit "C" - N & MB3N303 & N303 & 0.685 & 0.008 & 8.51 & 0.027 & 0.586 & 3.64 & 0.385 & 24.4 & $<0.002$ \\
\hline 4 & 2 & 16 & $\operatorname{mr} 12 \operatorname{lm} 22$ & Frit “Bone2"-U & MB3U326 & U326 & 0.666 & 0.008 & 8.25 & 0.498 & 0.565 & 10.3 & 0.385 & 25.7 & $<0.002$ \\
\hline 4 & 2 & 17 & stdlm 423 & Batch 1 & Batch 1 & Batch 1 & 0.917 & 0.075 & 9.59 & 0.882 & 1.31 & 6.82 & 0.566 & 24.1 & 0.091 \\
\hline
\end{tabular}


Immobilization Technology Section

WSRC-TR-2001-00131

Savannah River Technology Center

Rev. 0

Westinghouse Savannah River Company

Table E2: SRTC-ML Measurements for Samples Prepared

Using the Peroxide Fusion Method

(Values are in elemental weight percents, wt\%'s.)

\begin{tabular}{|c|c|c|c|c|c|c|c|c|c|c|}
\hline Block & Sub-Block & Sequence (pf) & SRTC-ML ID (pf) & Glass/Frit ID & Glass ID & Shortened Glass ID & $\mathbf{A l}(\mathbf{v})$ & $\mathbf{B}(\mathbf{v})$ & $\operatorname{Li}(\mathbf{v})$ & $\mathbf{U}(\mathbf{v})$ \\
\hline 1 & 1 & 1 & stdpf 111 & Batch 1 & Batch 1 & Batch 1 & 2.55 & 2.49 & 2.03 & $<0.281$ \\
\hline 1 & 1 & 2 & $\operatorname{mr} 12 \mathrm{pf} 21$ & Frit "Bone2"-U & MB3U326 & U326 & 2.28 & 1.90 & 2.71 & 2.13 \\
\hline 1 & 1 & 3 & $\operatorname{mr} 15 \mathrm{pf} 11$ & Frit "N" - N & MB3N314 & N314 & 2.33 & 4.55 & 1.99 & 1.99 \\
\hline 1 & 1 & 4 & ustdpf 111 & Ustd & Ustd & Ustd & 2.12 & 2.84 & 1.37 & 1.92 \\
\hline 1 & 1 & 5 & $\mathrm{mr} 15 \mathrm{pf} 21$ & Frit "N" - N & MB3N314 & N314 & 2.34 & 4.63 & 2.01 & 2.03 \\
\hline 1 & 1 & 6 & mr09pf 21 & Frit "Mimi" - N & MB3N322 & N322 & 2.31 & 1.85 & 1.72 & 2.13 \\
\hline 1 & 1 & 7 & $\mathrm{mr} 25 \mathrm{pf} 11$ & Frit "T" - N & MB3N320 & N320 & 2.31 & 1.85 & 2.69 & 2.21 \\
\hline 1 & 1 & 8 & stdpf 112 & Batch 1 & Batch 1 & Batch 1 & 2.55 & 2.51 & 2.03 & $<0.281$ \\
\hline 1 & 1 & 9 & $\mathrm{mr} 25 \mathrm{pf} 21$ & Frit "T" - N & MB3N320 & N320 & 2.34 & 1.84 & 2.70 & 2.24 \\
\hline 1 & 1 & 10 & mr03pf11 & Frit KMA-2A-N & MB3N324 & N324 & 2.32 & 3.45 & 2.81 & 2.26 \\
\hline 1 & 1 & 11 & $\operatorname{mr} 12 \mathrm{pf} 11$ & Frit "Bone2"-U & MB3U326 & U326 & 2.29 & 1.89 & 2.72 & 2.13 \\
\hline 1 & 1 & 12 & $\mathrm{mr} 22 \mathrm{pf} 21$ & Frit "Mimi" - U & MB3U322 & $\mathrm{U} 322$ & 2.23 & 1.81 & 1.67 & 2.06 \\
\hline 1 & 1 & 13 & ustdpf 112 & Ustd & Ustd & Ustd & 2.14 & 2.90 & 1.38 & 1.96 \\
\hline 1 & 1 & 14 & mr06pf 21 & Frit "O" - N & MB3N315 & N315 & 2.38 & 4.71 & 3.47 & 2.36 \\
\hline 1 & 1 & 15 & $\mathrm{mr} 22 \mathrm{pf} 11$ & Frit "Mimi" - U & MB3U322 & U322 & 2.24 & 1.85 & 1.71 & 2.06 \\
\hline 1 & 1 & 16 & mr06pf11 & Frit "O" - N & MB3N315 & N315 & 2.34 & 4.67 & 3.41 & 2.32 \\
\hline 1 & 1 & 17 & mr09pf11 & Frit "Mimi" - N & MB3N322 & N322 & 2.29 & 1.90 & 1.72 & 2.13 \\
\hline 1 & 1 & 18 & mr03pf 21 & Frit KMA-2A-N & MB3N324 & N324 & 2.33 & 3.62 & 2.86 & 2.28 \\
\hline 1 & 1 & 19 & stdpf 113 & Batch 1 & Batch 1 & Batch 1 & 2.57 & 2.62 & 2.05 & $<0.281$ \\
\hline 1 & 2 & 1 & stdpf121 & Batch 1 & Batch 1 & Batch 1 & 2.57 & 2.50 & 2.05 & $<0.281$ \\
\hline 1 & 2 & 2 & mr09pf22 & Frit "Mimi" - N & MB3N322 & N322 & 2.34 & 1.81 & 1.74 & 2.21 \\
\hline 1 & 2 & 3 & $\operatorname{mr} 12 \mathrm{pf} 22$ & Frit "Bone2"-U & MB3U326 & U326 & 2.29 & 1.86 & 2.73 & 2.20 \\
\hline 1 & 2 & 4 & ustdpf 121 & Ustd & Ustd & Ustd & 2.16 & 2.86 & 1.39 & 1.99 \\
\hline 1 & 2 & 5 & $\mathrm{mr} 12 \mathrm{pf} 12$ & Frit "Bone2"-U & MB3U326 & U326 & 2.29 & $\begin{array}{l}2.00 \\
1.89\end{array}$ & 2.74 & 2.18 \\
\hline 1 & 2 & 6 & mr03pf12 & Frit KMA-2A-N & MB3N324 & N324 & 2.33 & 3.54 & 2.85 & 2.29 \\
\hline 1 & 2 & 7 & mr03pf 22 & Frit KMA-2A-N & MB3N324 & N324 & 2.33 & 3.50 & 2.86 & 2.31 \\
\hline 1 & 2 & 8 & stdpf122 & Batch 1 & Batch 1 & Batch 1 & 2.57 & 2.51 & 2.05 & $<0.281$ \\
\hline 1 & 2 & 9 & mr $15 p f 12$ & Frit "N" - N & MB3N314 & N314 & 2.36 & 4.67 & 2.03 & 2.07 \\
\hline 1 & 2 & 10 & $\mathrm{mr} 22 \mathrm{pf} 22$ & Frit "Mimi" - U & MB3U322 & U322 & 2.26 & $\begin{array}{l}4.07 \\
1.81\end{array}$ & $\begin{array}{l}2.05 \\
1.69\end{array}$ & 2.07 \\
\hline 1 & 2 & 11 & $\mathrm{mr} 25 \mathrm{pf} 12$ & Frit "T" - N & MB3N320 & N320 & 2.38 & $\begin{array}{l}1.01 \\
1.87\end{array}$ & 2.77 & 2.25 \\
\hline 1 & 2 & 12 & mr06pf12 & Frit "O" - N & MB3N315 & N315 & 2.36 & 4.63 & 3.45 & 2.31 \\
\hline 1 & 2 & 13 & ustdpf 122 & Ustd & Ustd & Ustd & 2.2 & 2.95 & 1.41 & 2.02 \\
\hline 1 & 2 & 14 & mr06pf 22 & Frit "O" - N & MB3N315 & N315 & 2.39 & 4.62 & 3.47 & 2.42 \\
\hline 1 & 2 & 15 & $\mathrm{mr} 25 \mathrm{pf} 22$ & Frit "T" - N & MB3N320 & N320 & 2.34 & 1.79 & 2.70 & 2.35 \\
\hline 1 & 2 & 16 & mr09pf12 & Frit "Mimi" - N & MB3N322 & N322 & 2.28 & 1.75 & 1.70 & 2.21 \\
\hline 1 & 2 & 17 & mr15pf 22 & Frit "N" - N & MB3N314 & N314 & 2.35 & 4.60 & 2.01 & 2.08 \\
\hline 1 & 2 & 18 & $\mathrm{mr} 22 \mathrm{p} 12$ & Frit "Mimi" - U & MB3U322 & U322 & 2.24 & 1.85 & 1.70 & 2.10 \\
\hline 1 & 2 & 19 & stdpf 123 & Batch 1 & Batch 1 & Batch 1 & 2.6 & 2.53 & 2.06 & $<0.281$ \\
\hline 2 & 1 & 1 & stdpf 211 & Batch 1 & Batch 1 & Batch 1 & 2.56 & 2.49 & 2.06 & $<0.281$ \\
\hline 2 & 1 & 2 & mr16pf11 & Frit $165-\mathrm{U}$ & MB3U165 & U165 & 2.21 & 2.25 & 2.41 & 1.86 \\
\hline 2 & 1 & 3 & $\mathrm{mr} 21 \mathrm{pf} 21$ & Frit $200-\mathrm{N}$ & MB3N200 & $\mathrm{N} 200$ & 2.33 & 2.71 & 1.71 & 2.20 \\
\hline 2 & 1 & 4 & ustdpf 211 & Ustd & Ustd & Ustd & 2.14 & 2.79 & 1.39 & 1.94 \\
\hline 2 & 1 & 5 & mr16pf 21 & Frit 165 - U & MB3U165 & U165 & 2.19 & 2.26 & 2.39 & 1.87 \\
\hline 2 & 1 & 6 & mr26pf11 & Frit KMA-2-N & MB3N323 & N323 & 2.32 & 3.37 & 1.78 & 2.21 \\
\hline 2 & 1 & 7 & $\mathrm{mr} 11 \mathrm{pf} 21$ & Frit $165-\mathrm{N}$ & MB3N165 & N165 & 2.32 & 2.26 & 2.36 & 1.95 \\
\hline 2 & 1 & 8 & stdpf 212 & Batch 1 & Batch 1 & Batch 1 & 2.52 & 2.39 & 2.04 & $<0.281$ \\
\hline 2 & 1 & 9 & $\mathrm{mr} 26 \mathrm{pf} 21$ & Frit KMA-2-N & MB3N323 & N323 & 2.31 & 3.31 & 1.77 & 2.20 \\
\hline 2 & 1 & 10 & mr14pf 21 & Frit "G" - U & MB3U307 & U307 & 2.31 & 2.71 & 3.68 & 2.08 \\
\hline 2 & 1 & 11 & mr07pf11 & Frit "M" - N & MB3N313 & N313 & 2.25 & 1.46 & 3.61 & 2.00 \\
\hline 2 & 1 & 12 & $\mathrm{mr} 07 \mathrm{pf} 21$ & Frit "M" - N & MB3N313 & N313 & 2.28 & 1.49 & 3.67 & 2.02 \\
\hline 2 & 1 & 13 & ustdpf 212 & Ustd & Ustd & Ustd & 2.11 & 2.79 & 1.39 & 1.89 \\
\hline 2 & 1 & 14 & $\mathrm{mr} 21 \mathrm{pf} 11$ & Frit $200-\mathrm{N}$ & MB3N200 & N200 & 2.35 & 2.75 & 1.72 & 2.17 \\
\hline 2 & 1 & 15 & $\mathrm{mr} 14 \mathrm{pf} 11$ & Frit "G" - U & MB3U307 & U307 & 2.33 & 2.76 & 3.70 & 2.13 \\
\hline 2 & 1 & 16 & $\mathrm{mr} 11 \mathrm{pf} 11$ & Frit $165-\mathrm{N}$ & MB3N165 & N165 & 2.36 & 2.23 & 2.39 & 1.99 \\
\hline 2 & 1 & 17 & stdpf 213 & Batch 1 & Batch 1 & Batch 1 & 2.6 & 2.41 & 2.06 & $<0.281$ \\
\hline 2 & 2 & 1 & stdpf 221 & Batch 1 & Batch 1 & Batch 1 & 2.58 & 2.46 & 2.07 & $<0.281$ \\
\hline 2 & 2 & 2 & $\operatorname{mr} 11 \mathrm{pf} 22$ & Frit $165-\mathrm{N}$ & MB3N165 & N165 & 2.37 & 2.28 & 2.39 & 2.05 \\
\hline 2 & 2 & 3 & $\operatorname{mr} 11 \mathrm{pf} 12$ & Frit $165-\mathrm{N}$ & MB3N165 & N165 & 2.41 & 2.28 & 2.40 & 2.11 \\
\hline 2 & 2 & 4 & ustdpf 221 & Ustd & Ustd & Ustd & 2.19 & 2.89 & 1.41 & 2.02 \\
\hline 2 & 2 & 5 & $\mathrm{mr} 26 \mathrm{pf} 22$ & Frit KMA-2-N & MB3N323 & N323 & 2.38 & 3.51 & 1.81 & 2.33 \\
\hline 2 & 2 & 6 & mr26pf12 & Frit KMA-2-N & MB3N323 & N323 & 2.39 & 3.50 & 1.81 & 2.37 \\
\hline 2 & 2 & 7 & $\operatorname{mr} 16 \mathrm{pf} 22$ & Frit $165-\mathrm{U}$ & MB3U165 & U165 & 2.25 & 2.29 & 2.44 & 1.98 \\
\hline 2 & 2 & 8 & stdpf 222 & Batch 1 & Batch 1 & Batch 1 & 2.61 & 2.49 & 2.09 & $<0.281$ \\
\hline 2 & 2 & 9 & $\mathrm{mr} 14 \mathrm{pf} 12$ & Frit "G" - U & MB3U307 & U307 & 2.4 & 2.76 & 3.74 & 2.31 \\
\hline 2 & 2 & 10 & $\operatorname{mr} 16 \mathrm{pf} 12$ & Frit $165-U$ & MB3U165 & U165 & 2.25 & 2.25 & 2.44 & 2.00 \\
\hline 2 & 2 & 11 & $\operatorname{mr} 14 \mathrm{pf} 22$ & Frit "G" - U & MB3U307 & U307 & 2.38 & 2.73 & 3.73 & 2.29 \\
\hline 2 & 2 & 12 & mr07pf 22 & Frit "M" - N & MB3N313 & N313 & 2.38 & 1.52 & 3.77 & 2.25 \\
\hline 2 & 2 & 13 & ustdpf 222 & Ustd & Ustd & Ustd & 2.19 & 2.87 & 1.41 & 2.05 \\
\hline 2 & 2 & 14 & $\mathrm{mr} 21 \mathrm{pf} 22$ & Frit $200-\mathrm{N}$ & MB3N200 & $\mathrm{N} 200$ & 2.39 & 2.84 & 1.75 & 2.38 \\
\hline 2 & 2 & 15 & $\mathrm{mr} 21 \mathrm{pf} 12$ & Frit $200-\mathrm{N}$ & MB3N200 & $\mathrm{N} 200$ & 2.42 & 2.83 & 1.74 & 2.35 \\
\hline 2 & 2 & 16 & $\mathrm{mr} 07 \mathrm{pf} 12$ & Frit "M" - N & MB3N313 & N313 & 2.35 & 1.52 & 3.72 & 2.27 \\
\hline 2 & 2 & 17 & stdp223 & Batch 1 & Batch 1 & Batch 1 & 2.62 & 2.47 & 2.09 & $<0.281$ \\
\hline
\end{tabular}


Immobilization Technology Section

WSRC-TR-2001-00131

Savannah River Technology Center

Rev. 0

Westinghouse Savannah River Company

Table E2: SRTC-ML Measurements for Samples Prepared

Using the Peroxide Fusion Method (continued)

(Values are in elemental weight percents, wt\%'s.)

\begin{tabular}{|c|c|c|c|c|c|c|c|c|c|c|}
\hline Block & Sub-Block & Sequence (pf) & SRTC-ML ID (pf) & Glass/Frit ID & Glass ID & Shortened Glass ID & $\mathbf{A l}(\mathbf{v})$ & $\mathbf{B}(\mathbf{v})$ & $\operatorname{Li}(\mathbf{v})$ & $\mathbf{U}(\mathbf{v})$ \\
\hline 3 & 1 & 1 & stdpf 311 & Batch 1 & Batch 1 & Batch 1 & 2.59 & 2.45 & 2.08 & $<0.281$ \\
\hline 3 & 1 & 2 & mr04pf11 & Frit "Bone2"-N & MB3N326 & N326 & 2.51 & 1.92 & 2.94 & 2.47 \\
\hline 3 & 1 & 3 & mr10pf11 & Frit "C" - N & MB3N303 & N303 & 3.27 & 4.57 & 3.5 & 2.41 \\
\hline 3 & 1 & 4 & ustdpf 311 & Ustd & Ustd & Ustd & 2.15 & 2.79 & 1.39 & 2.02 \\
\hline 3 & 1 & 5 & $\operatorname{mr} 10 \mathrm{pf} 21$ & Frit "C" - N & MB3N303 & N303 & 3.34 & 4.74 & 3.58 & 2.49 \\
\hline 3 & 1 & 6 & mr23pf11 & Frit KMA-2-U & MB3U323 & U323 & 2.41 & 3.53 & 1.83 & 2.25 \\
\hline 3 & 1 & 7 & $\mathrm{mr} 13 \mathrm{pf} 21$ & Frit KMA-2A-U & MB3U324 & U324 & 2.27 & 3.29 & 2.77 & 2.18 \\
\hline 3 & 1 & 8 & stdpf312 & Batch 1 & Batch 1 & Batch 1 & 2.57 & 2.43 & 2.04 & $<0.281$ \\
\hline 3 & 1 & 9 & $\operatorname{mr} 17 \mathrm{pf} 11$ & Frit "T" - U & MB3U320 & U320 & 2.46 & 1.92 & 2.95 & 2.32 \\
\hline 3 & 1 & 10 & mr13pf11 & Frit KMA-2A-U & MB3U324 & U324 & 2.39 & 3.41 & 2.92 & 2.29 \\
\hline 3 & 1 & 11 & mr05pf11 & Frit "C" - U & MB3U303 & U303 & 3.44 & 4.78 & 3.73 & 2.40 \\
\hline 3 & 1 & 12 & mr05pf 21 & Frit "C" - U & MB3U303 & U303 & 3.24 & 4.54 & 3.52 & 2.26 \\
\hline 3 & 1 & 13 & ustdpf 312 & Ustd & Ustd & Ustd & 2.14 & 2.79 & 1.39 & 2.01 \\
\hline 3 & 1 & 14 & mr02pf11 & Frit "M" - U & MB3U313 & U313 & 2.28 & 1.56 & 3.63 & 2.17 \\
\hline 3 & 1 & 15 & $\mathrm{mr} 23 \mathrm{pf} 21$ & Frit KMA-2-U & MB3U323 & U323 & 2.38 & 3.48 & 1.80 & 2.21 \\
\hline 3 & 1 & 16 & mr02pf 21 & Frit "M" - U & MB3U313 & U313 & 2.3 & 1.55 & 3.68 & 2.20 \\
\hline 3 & 1 & 17 & $\mathrm{mr} 17 \mathrm{pf} 21$ & Frit “T" - U & MB3U320 & U320 & 2.28 & 1.82 & 2.74 & 2.15 \\
\hline 3 & 1 & 18 & mr04pf 21 & Frit "Bone2"-N & MB3N326 & N326 & 2.48 & 1.93 & 2.91 & 2.42 \\
\hline 3 & 1 & 19 & stdpf313 & Batch 1 & Batch 1 & Batch 1 & 2.59 & 2.49 & 2.08 & $<0.281$ \\
\hline 3 & 2 & 1 & stdpf321 & Batch 1 & Batch 1 & Batch 1 & 2.56 & 2.49 & 2.07 & $<0.281$ \\
\hline 3 & 2 & 2 & $\mathrm{mr02pf12}$ & Frit "M" - U & MB3U313 & U313 & 2.27 & 1.56 & 3.65 & 2.06 \\
\hline 3 & 2 & 3 & $\mathrm{mr} 02 \mathrm{pf} 22$ & Frit "M" - U & MB3U313 & $\mathrm{U} 313$ & 2.29 & 1.54 & 3.70 & 2.08 \\
\hline 3 & 2 & 4 & ustdpf 321 & Ustd & Ustd & Ustd & 2.16 & 2.81 & 1.42 & 1.96 \\
\hline 3 & 2 & 5 & $\operatorname{mr} 13 \mathrm{pf} 22$ & Frit KMA-2A-U & MB3U324 & U324 & 2.3 & 3.36 & 2.84 & 2.13 \\
\hline 3 & 2 & 6 & mr04pf 22 & Frit "Bone2"-N & MB3N326 & N326 & 2.31 & 1.82 & 2.76 & 2.24 \\
\hline 3 & 2 & 7 & $\mathrm{mr} 10 \mathrm{pf} 12$ & Frit "C" - N & MB3N303 & N303 & 3.22 & 4.57 & 3.48 & 2.32 \\
\hline 3 & 2 & 8 & stdpf 322 & Batch 1 & Batch 1 & Batch 1 & 2.59 & 2.48 & 2.09 & $<0.281$ \\
\hline 3 & 2 & 9 & $\mathrm{mr} 10 \mathrm{pf} 22$ & Frit "C" $-\mathrm{N}$ & MB3N303 & N303 & 3.24 & 4.65 & 3.49 & 2.35 \\
\hline 3 & 2 & 10 & $\mathrm{mr} 17 \mathrm{pf} 22$ & Frit "T" - U & MB3U320 & U320 & 2.29 & 1.85 & 2.77 & 2.11 \\
\hline 3 & 2 & 11 & $\mathrm{mr} 23 \mathrm{pf} 12$ & Frit KMA-2-U & MB3U323 & U323 & 2.29 & 3.33 & 1.76 & 2.11 \\
\hline 3 & 2 & 12 & mr05pf12 & Frit "C" - U & MB3U303 & U303 & 3.26 & 4.51 & 3.55 & 2.22 \\
\hline 3 & 2 & 13 & ustdpf 322 & Ustd & Ustd & Ustd & 2.18 & 2.82 & 1.41 & 2.00 \\
\hline 3 & 2 & 14 & mr05pf12 & Frit "C" - U & MB3U303 & U303 & 3.25 & 4.60 & 3.54 & 2.22 \\
\hline 3 & 2 & 15 & $\operatorname{mr} 17 \mathrm{pf} 12$ & Frit "T" - U & MB3U320 & $\mathrm{U} 320$ & 2.24 & 1.80 & 2.72 & 2.08 \\
\hline 3 & 2 & 16 & mr04pf12 & Frit "Bone2"-N & MB3N326 & N326 & 2.27 & 1.77 & 2.69 & 2.22 \\
\hline 3 & 2 & 17 & $\operatorname{mr} 13 \mathrm{pf} 12$ & Frit KMA-2A-U & MB3U324 & U324 & 2.35 & 3.43 & 2.88 & 2.21 \\
\hline 3 & 2 & 18 & $\mathrm{mr} 23 \mathrm{pf} 22$ & Frit KMA-2-U & MB3U323 & U323 & 2.35 & 3.41 & 1.79 & 2.13 \\
\hline 3 & 2 & 19 & stdpf 323 & Batch 1 & Batch 1 & Batch 1 & 2.61 & $\begin{array}{l}3.41 \\
2.52\end{array}$ & 2.11 & $<0.281$ \\
\hline 4 & 1 & 1 & stdp411 & Batch 1 & Batch 1 & Batch 1 & 2.47 & 2.32 & 1.99 & $<0.281$ \\
\hline 4 & 1 & 2 & $\mathrm{mr} 20 \mathrm{pf} 21$ & Frit "D" - U & MB3U304 & U304 & 3.21 & 1.53 & 2.04 & 2.20 \\
\hline 4 & 1 & 3 & mr08pf21 & Frit "O" - U & MB3U315 & U315 & 2.48 & 4.71 & 3.72 & 2.38 \\
\hline 4 & 1 & 4 & ustdpf411 & Ustd & Ustd & Ustd & 2.13 & 2.82 & 1.38 & 1.99 \\
\hline 4 & 1 & 5 & $\mathrm{mr} 18 \mathrm{pf} 21$ & Frit $200-\mathrm{U}$ & MB3U200 & U200 & 2.29 & 2.72 & 1.68 & 2.28 \\
\hline 4 & 1 & 6 & mr19pf11 & Frit "D" - N & MB3N304 & N304 & 3.42 & 1.66 & 2.14 & 2.39 \\
\hline 4 & 1 & 7 & mr01pf11 & Frit "N" - U & MB3U314 & U314 & 2.13 & 3.98 & 1.83 & 2.06 \\
\hline 4 & 1 & 8 & stdpf412 & Batch 1 & Batch 1 & Batch 1 & 2.57 & 2.52 & 2.07 & $<0.281$ \\
\hline 4 & 1 & 9 & $\mathrm{mr} 24 \mathrm{pf} 11$ & Frit "G" - N & MB3N307 & N307 & 2.47 & 2.99 & 3.90 & 2.39 \\
\hline 4 & 1 & 10 & $\mathrm{mr} 24 \mathrm{pf} 21$ & Frit "G" - N & MB3N307 & N307 & 2.45 & 2.98 & 3.86 & 2.37 \\
\hline 4 & 1 & 11 & mr20pf11 & Frit "D" - U & MB3U304 & U304 & 3.31 & 1.65 & 2.09 & 2.27 \\
\hline 4 & 1 & 12 & mr01pf 21 & Frit "N" - U & MB3U314 & U314 & 2.17 & 4.05 & 1.84 & 2.09 \\
\hline 4 & 1 & 13 & ustdpf 412 & Ustd & Ustd & Ustd & 2.18 & 2.93 & 1.39 & 2.05 \\
\hline 4 & 1 & 14 & $\operatorname{mr} 18 \mathrm{pf} 11$ & Frit $200-\mathrm{U}$ & MB3U200 & U200 & 2.31 & 2.82 & 1.78 & 2.30 \\
\hline 4 & 1 & 15 & mr08pf11 & Frit "O" - U & MB3U315 & U315 & 2.38 & 4.67 & 3.55 & 2.26 \\
\hline 4 & 1 & 16 & $\mathrm{mr} 19 \mathrm{pf} 21$ & Frit "D" - N & MB3N304 & N304 & 3.24 & 1.59 & 2.03 & 2.31 \\
\hline 4 & 1 & 17 & stdpf 413 & Batch 1 & Batch 1 & Batch 1 & 2.58 & 2.53 & 2.08 & $<0.281$ \\
\hline 4 & 2 & 1 & stdpf 421 & Batch 1 & Batch 1 & Batch 1 & 2.52 & 2.45 & 2.03 & $<0.281$ \\
\hline 4 & 2 & 2 & mr01pf 22 & Frit "N" - U & MB3U314 & U314 & 2.22 & 4.08 & 1.87 & 2.17 \\
\hline 4 & 2 & 3 & $\mathrm{mr} 18 \mathrm{pf} 12$ & Frit $200-\mathrm{U}$ & MB3U200 & U200 & 2.34 & 2.84 & 1.79 & 2.14 \\
\hline 4 & 2 & 4 & ustdpf 421 & Ustd & Ustd & Ustd & 2.15 & 2.73 & 1.37 & 1.98 \\
\hline 4 & 2 & 5 & $\mathrm{mr} 19 \mathrm{pf} 12$ & Frit "D" - N & MB3N304 & N304 & 3.16 & 1.51 & 1.95 & 2.22 \\
\hline 4 & 2 & 6 & $\mathrm{mr} 20 \mathrm{pf} 22$ & Frit "D" - U & MB3U304 & U304 & 3.04 & 1.45 & 1.90 & 2.05 \\
\hline 4 & 2 & 7 & $\mathrm{mr} 24 \mathrm{pf} 22$ & Frit "G" - N & MB3N307 & N307 & 2.34 & 2.68 & 3.66 & 2.23 \\
\hline 4 & 2 & 8 & stdpf 422 & Batch 1 & Batch 1 & Batch 1 & 2.51 & 2.37 & 2.02 & $<0.281$ \\
\hline 4 & 2 & 9 & mr08pf12 & Frit "O" - U & MB3U315 & U315 & 2.28 & 4.32 & 3.39 & 2.13 \\
\hline 4 & 2 & 10 & mr20pf12 & Frit "D" - U & MB3U304 & U304 & 3.06 & 1.47 & 1.93 & 2.06 \\
\hline 4 & 2 & 11 & $\operatorname{mr} 18 \mathrm{pf} 22$ & Frit $200-U$ & MB3U200 & $\mathrm{U} 200$ & 2.34 & 2.71 & 1.70 & 2.34 \\
\hline 4 & 2 & 12 & $\mathrm{mr} 19 \mathrm{pf} 22$ & Frit "D" - N & MB3N304 & N304 & 3.21 & 1.54 & 2.01 & 2.24 \\
\hline 4 & 2 & 13 & ustdpf 422 & Ustd & Ustd & Ustd & 2.14 & 2.76 & 1.37 & 2.00 \\
\hline 4 & 2 & 14 & $\mathrm{mr} 24 \mathrm{pf} 12$ & Frit "G" - N & MB3N307 & N307 & 2.35 & 2.74 & 3.67 & 2.23 \\
\hline 4 & 2 & 15 & mr01pf12 & Frit "N" - U & MB3U314 & U314 & 2.18 & 4.04 & 1.86 & 2.12 \\
\hline 4 & 2 & 16 & mr08pf 22 & Frit "O" - U & MB3U315 & U315 & 2.27 & 4.32 & 3.36 & 2.13 \\
\hline 4 & 2 & 17 & stdpf 423 & Batch 1 & Batch 1 & Batch 1 & 2.59 & 2.45 & 2.07 & $<0.281$ \\
\hline
\end{tabular}


Immobilization Technology Section

WSRC-TR-2001-00131

Savannah River Technology Center

Rev. 0

Westinghouse Savannah River Company

Table E3: Composition of PCT Leachate Solutions for Glasses from Nominally Washed Sludge.

\begin{tabular}{|c|c|c|c|c|c|c|c|c|c|c|c|c|c|c|c|}
\hline & & & & \multirow{2}{*}{\multicolumn{4}{|c|}{$\begin{array}{c}\text { Concentrations in ppm } \\
\text { (as reported) }\end{array}$}} & \multicolumn{4}{|c|}{ Concentrations in ppm } & \multirow{2}{*}{\multicolumn{4}{|c|}{ Common Logarithm of ppm Concentrations }} \\
\hline Glass & Lab & & & & & & & & $r$ correct & for dilut & & & & & \\
\hline ID & ID & Blk & Seq & B & $\mathbf{L i}$ & $\mathrm{Na}$ & $\mathrm{Si}$ & B & $\mathbf{L i}$ & $\mathrm{Na}$ & Si & $\log [\mathrm{B}]$ & $\log [\mathrm{Li}]$ & $\log [\mathrm{Na}]$ & $\log [\mathrm{Si}]$ \\
\hline std & std-b1-1 & 1 & 1 & 20.40 & 10.80 & 75.60 & 50.30 & 20.40 & 10.80 & 75.60 & 50.30 & 1.3096 & 1.0334 & 1.8785 & 1.7016 \\
\hline MB3N303q & ga58 & 1 & 2 & 22.30 & 19.00 & 8.61 & 74.10 & 37.17 & 31.67 & 14.35 & 123.50 & 1.5702 & 1.5006 & 1.1569 & 2.0917 \\
\hline MB3N304clc & ga18 & 1 & 3 & 10.70 & 16.10 & 126.00 & 138.00 & 17.83 & 26.83 & 210.00 & 230.00 & 1.2512 & 1.4287 & 2.3222 & 2.3617 \\
\hline MB3N324clc & ga78 & 1 & 4 & 18.80 & 17.70 & 24.30 & 87.70 & 31.33 & 29.50 & 40.50 & 146.17 & 1.4960 & 1.4698 & 1.6075 & 2.1649 \\
\hline MB3N304q & ga63 & 1 & 5 & 11.00 & 15.60 & 132.00 & 135.00 & 18.33 & 26.00 & 220.00 & 225.00 & 1.2633 & 1.4150 & 2.3424 & 2.3522 \\
\hline blank & ga41 & 1 & 6 & 0.53 & $<0.040$ & 0.95 & $<0.790$ & 0.88 & 0.03 & 1.59 & 0.66 & -0.0563 & -1.4771 & 0.2010 & -0.1816 \\
\hline MB3N322q & ga55 & 1 & 7 & 6.85 & 8.22 & 32.50 & 81.20 & 11.42 & 13.70 & 54.17 & 135.34 & 1.0575 & 1.1367 & 1.7337 & 2.1314 \\
\hline MB3N326q & ga75 & 1 & 8 & 12.90 & 20.00 & 62.80 & 122.00 & 21.50 & 33.33 & 104.67 & 203.34 & 1.3324 & 1.5229 & 2.0198 & 2.3082 \\
\hline MB3N323q & ga64 & 1 & 9 & 15.50 & 9.51 & 26.40 & 72.50 & 25.83 & 15.85 & 44.00 & 120.84 & 1.4122 & 1.2000 & 1.6435 & 2.0822 \\
\hline std & std-b1-2 & 1 & 10 & 20.80 & 10.50 & 72.80 & 52.00 & 20.80 & 10.50 & 72.80 & 52.00 & 1.3181 & 1.0212 & 1.8621 & 1.7160 \\
\hline MB3N313clc & ga21 & 1 & 11 & 17.20 & 61.20 & 80.10 & 286.00 & 28.67 & 102.00 & 133.50 & 476.68 & 1.4574 & 2.0086 & 2.1255 & 2.6782 \\
\hline MB3N303clc & ga83 & 1 & 12 & 21.70 & 17.50 & 9.17 & 75.40 & 36.17 & 29.17 & 15.28 & 125.67 & 1.5583 & 1.4649 & 1.1842 & 2.0992 \\
\hline EA & ga80 & 1 & 13 & 38.30 & 12.20 & 92.50 & 55.20 & 638.33 & 203.33 & 1541.67 & 920.00 & 2.8050 & 2.3082 & 3.1880 & 2.9638 \\
\hline MB3N323clc & ga37 & 1 & 14 & 13.50 & 8.62 & 24.70 & 67.40 & 22.50 & 14.37 & 41.17 & 112.34 & 1.3522 & 1.1574 & 1.6146 & 2.0505 \\
\hline MB3N326clc & ga06 & 1 & 15 & 13.10 & 21.90 & 62.70 & 127.00 & 21.83 & 36.50 & 104.50 & 211.67 & 1.3391 & 1.5623 & 2.0191 & 2.3257 \\
\hline MB3N322clc & ga16 & 1 & 16 & 6.78 & 7.91 & 29.90 & 80.10 & 11.30 & 13.18 & 49.83 & 133.50 & 1.0531 & 1.1200 & 1.6975 & 2.1255 \\
\hline MB3N313q & ga25 & 1 & 17 & 12.50 & 34.10 & 65.30 & 168.00 & 20.83 & 56.83 & 108.84 & 280.01 & 1.3188 & 1.7546 & 2.0368 & 2.4472 \\
\hline MB3N324q & ga28 & 1 & 18 & 20.20 & 18.50 & 24.40 & 90.50 & 33.67 & 30.83 & 40.67 & 150.84 & 1.5272 & 1.4890 & 1.6092 & 2.1785 \\
\hline std & std-b1-3 & 1 & 19 & 21.10 & 10.70 & 73.70 & 52.90 & 21.10 & 10.70 & 73.70 & 52.90 & 1.3243 & 1.0294 & 1.8675 & 1.7235 \\
\hline std & std-b2-1 & 2 & 1 & 18.10 & 10.20 & 75.80 & 45.60 & 18.10 & 10.20 & 75.80 & 45.60 & 1.2577 & 1.0086 & 1.8797 & 1.6590 \\
\hline MB3N326clc & ga50 & 2 & 2 & 11.50 & 21.50 & 66.20 & 115.00 & 19.17 & 35.83 & 110.34 & 191.67 & 1.2826 & 1.5543 & 2.0427 & 2.2826 \\
\hline MB3N304clc & ga59 & 2 & 3 & 9.22 & 15.30 & 130.00 & 123.00 & 15.37 & 25.50 & 216.67 & 205.00 & 1.1866 & 1.4065 & 2.3358 & 2.3118 \\
\hline MB3N323clc & ga62 & 2 & 4 & 12.10 & 8.82 & 26.20 & 62.60 & 20.17 & 14.70 & 43.67 & 104.34 & 1.3046 & 1.1673 & 1.6402 & 2.0184 \\
\hline MB3N324clc & ga67 & 2 & 5 & 17.70 & 18.30 & 26.10 & 80.80 & 29.50 & 30.50 & 43.50 & 134.67 & 1.4698 & 1.4843 & 1.6385 & 2.1293 \\
\hline MB3N326q & ga10 & 2 & 6 & 11.20 & 20.10 & 68.90 & 111.00 & 18.67 & 33.50 & 114.84 & 185.00 & 1.2711 & 1.5251 & 2.0601 & 2.2672 \\
\hline MB3N322q & ga39 & 2 & 7 & 5.85 & 8.38 & 34.30 & 75.00 & 9.75 & 13.97 & 57.17 & 125.00 & 0.9890 & 1.1451 & 1.7572 & 2.0969 \\
\hline MB3N304q & ga17 & 2 & 8 & 9.50 & 15.20 & 139.00 & 123.00 & 15.83 & 25.33 & 231.67 & 205.00 & 1.1996 & 1.4037 & 2.3649 & 2.3118 \\
\hline EA & ga53 & 2 & 9 & 35.10 & 12.10 & 97.80 & 51.60 & 585.00 & 201.67 & 1630.00 & 860.00 & 2.7672 & 2.3046 & 3.2122 & 2.9345 \\
\hline std & std-b2-2 & 2 & 10 & 19.10 & 10.70 & 79.00 & 47.90 & 19.10 & 10.70 & 79.00 & 47.90 & 1.2810 & 1.0294 & 1.8976 & 1.6803 \\
\hline MB3N303clc & ga22 & 2 & 11 & 19.70 & 17.80 & 9.95 & 68.30 & 32.83 & 29.67 & 16.58 & 113.84 & 1.5163 & 1.4723 & 1.2197 & 2.0563 \\
\hline MB3N323q & ga65 & 2 & 12 & 13.70 & 9.46 & 26.90 & 65.40 & 22.83 & 15.77 & 44.83 & 109.00 & 1.3586 & 1.1977 & 1.6516 & 2.0374 \\
\hline MB3N313q & ga15 & 2 & 13 & 11.10 & 32.50 & 65.80 & 153.00 & 18.50 & 54.17 & 109.67 & 255.01 & 1.2672 & 1.7337 & 2.0401 & 2.4065 \\
\hline MB3N322clc & ga20 & 2 & 14 & 6.26 & 8.40 & 33.40 & 79.70 & 10.43 & 14.00 & 55.67 & 132.84 & 1.0184 & 1.1461 & 1.7456 & 2.1233 \\
\hline MB3N313clc & ga47 & 2 & 15 & 15.80 & 59.90 & 83.90 & 269.00 & 26.33 & 99.84 & 139.84 & 448.34 & 1.4205 & 1.9993 & 2.1456 & 2.6516 \\
\hline MB3N324q & ga84 & 2 & 16 & 17.50 & 17.40 & 23.60 & 82.10 & 29.17 & 29.00 & 39.33 & 136.84 & 1.4649 & 1.4624 & 1.5948 & 2.1362 \\
\hline MB3N303q & ga23 & 2 & 17 & 21.40 & 18.80 & 8.00 & 72.50 & 35.67 & 31.33 & 13.33 & 120.84 & 1.5523 & 1.4960 & 1.1249 & 2.0822 \\
\hline std & std-b2-3 & 2 & 18 & 18.70 & 10.50 & 77.50 & 47.20 & 18.70 & 10.50 & 77.50 & 47.20 & 1.2718 & 1.0212 & 1.8893 & 1.6739 \\
\hline std & std-b3-1 & 3 & 1 & 18.30 & 10.40 & 75.80 & 43.90 & 18.30 & 10.40 & 75.80 & 43.90 & 1.2625 & 1.0170 & 1.8797 & 1.6425 \\
\hline MB3N326clc & ga76 & 3 & 2 & 11.20 & 21.10 & 63.30 & 108.00 & 18.67 & 35.17 & 105.50 & 180.00 & 1.2711 & 1.5461 & 2.0233 & 2.2553 \\
\hline EA & ga86 & 3 & 3 & 27.20 & 9.81 & 74.40 & 40.20 & 453.33 & 163.50 & 1240.00 & 670.00 & 2.6564 & 2.2135 & 3.0934 & 2.8261 \\
\hline MB3N322clc & ga79 & 3 & 4 & 6.28 & 8.38 & 33.20 & 78.10 & 10.47 & 13.97 & 55.33 & 130.17 & 1.0198 & 1.1451 & 1.7430 & 2.1145 \\
\hline MB3N303q & ga69 & 3 & 5 & 22.20 & 19.30 & 8.07 & 71.90 & 37.00 & 32.17 & 13.45 & 119.84 & 1.5682 & 1.5074 & 1.1287 & 2.0786 \\
\hline MB3N324q & ga12 & 3 & 6 & 18.00 & 17.20 & 24.00 & 79.10 & 30.00 & 28.67 & 40.00 & 131.84 & 1.4771 & 1.4574 & 1.6021 & 2.1200 \\
\hline MB3N326q & ga27 & 3 & 7 & 11.00 & 18.90 & 63.90 & 105.00 & 18.33 & 31.50 & 106.50 & 175.00 & 1.2633 & 1.4983 & 2.0274 & 2.2430 \\
\hline MB3N303clc & ga29 & 3 & 8 & 19.80 & 17.20 & 8.79 & 67.60 & 33.00 & 28.67 & 14.65 & 112.67 & 1.5185 & 1.4574 & 1.1658 & 2.0518 \\
\hline MB3N323clc & ga45 & 3 & 9 & 12.30 & 8.32 & 24.90 & 62.30 & 20.50 & 13.87 & 41.50 & 103.84 & 1.3118 & 1.1420 & 1.6181 & 2.0163 \\
\hline std & std-b3-2 & 3 & 10 & 19.40 & 10.70 & 77.80 & 46.90 & 19.40 & 10.70 & 77.80 & 46.90 & 1.2878 & 1.0294 & 1.8910 & 1.6712 \\
\hline MB3N313clc & ga26 & 3 & 11 & 15.00 & 59.30 & 80.90 & 200.00 & 25.00 & 98.84 & 134.84 & 333.34 & 1.3979 & 1.9949 & 2.1298 & 2.5229 \\
\hline MB3N322q & ga71 & 3 & 12 & 6.00 & 7.97 & 32.30 & 73.50 & 10.00 & 13.28 & 53.83 & 122.50 & 1.0000 & 1.1233 & 1.7311 & 2.0881 \\
\hline MB3N324clc & ga60 & 3 & 13 & 18.10 & 17.50 & 24.70 & 81.60 & 30.17 & 29.17 & 41.17 & 136.00 & 1.4795 & 1.4649 & 1.6146 & 2.1335 \\
\hline MB3N304q & ga36 & 3 & 14 & 9.88 & 14.80 & 132.00 & 115.00 & 16.47 & 24.67 & 220.00 & 191.67 & 1.2166 & 1.3921 & 2.3424 & 2.2826 \\
\hline MB3N313q & ga74 & 3 & 15 & 11.60 & 33.40 & 66.50 & 147.00 & 19.33 & 55.67 & 110.84 & 245.00 & 1.2863 & 1.7456 & 2.0447 & 2.3892 \\
\hline MB3N304clc & ga14 & 3 & 16 & 9.76 & 15.00 & 124.00 & 119.00 & 16.27 & 25.00 & 206.67 & 198.34 & 1.2113 & 1.3979 & 2.3153 & 2.2974 \\
\hline MB3N323q & ga24 & 3 & 17 & 14.30 & 9.47 & 27.30 & 65.20 & 23.83 & 15.78 & 45.50 & 108.67 & 1.3772 & 1.1982 & 1.6580 & 2.0361 \\
\hline std & std-b3-3 & 3 & 18 & 19.90 & 10.90 & 78.80 & 48.50 & 19.90 & 10.90 & 78.80 & 48.50 & 1.2989 & 1.0374 & 1.8965 & 1.6857 \\
\hline std & std-b4-1 & 4 & 1 & 18.80 & 10.50 & 77.00 & 47.80 & 18.80 & 10.50 & 77.00 & 47.80 & 1.2742 & 1.0212 & 1.8865 & 1.6794 \\
\hline MB3N200clc & ga66 & 4 & 2 & 12.10 & 8.78 & 41.80 & 71.30 & 20.17 & 14.63 & 69.67 & 118.84 & 1.3046 & 1.1654 & 1.8430 & 2.0749 \\
\hline MB3N200q & ga54 & 4 & 3 & 12.70 & 9.35 & 44.10 & 71.30 & 21.17 & 15.58 & 73.50 & 118.84 & 1.3257 & 1.1927 & 1.8663 & 2.0749 \\
\hline MB3N165clc & ga57 & 4 & 4 & 12.70 & 15.40 & 65.30 & 89.90 & 21.17 & 25.67 & 108.84 & 149.84 & 1.3257 & 1.4094 & 2.0368 & 2.1756 \\
\hline MB3N307q & ga49 & 4 & 5 & 16.10 & 31.80 & 31.30 & 129.00 & 26.83 & 53.00 & 52.17 & 215.00 & 1.4287 & 1.7243 & 1.7174 & 2.3324 \\
\hline MB3N165q & ga11 & 4 & 6 & 13.60 & 16.00 & 74.80 & 91.10 & 22.67 & 26.67 & 124.67 & 151.84 & 1.3554 & 1.4260 & 2.0958 & 2.1814 \\
\hline ARM & ga56 & 4 & 7 & 10.60 & 9.40 & 22.20 & 35.80 & 17.67 & 15.67 & 37.00 & 59.67 & 1.2472 & 1.1950 & 1.5682 & 1.7757 \\
\hline MB3N320clc & ga08 & 4 & 8 & 10.90 & 22.70 & 75.20 & 127.00 & 18.17 & 37.83 & 125.34 & 211.67 & 1.2593 & 1.5779 & 2.0981 & 2.3257 \\
\hline std & std-b4-2 & 4 & 9 & 18.80 & 10.80 & 79.30 & 48.40 & 18.80 & 10.80 & 79.30 & 48.40 & 1.2742 & 1.0334 & 1.8993 & 1.6848 \\
\hline
\end{tabular}

Notes:

(1). Values that were below detection (indicated by a "<") were converted to $1 / 2$ the detection limit.

(2) The shaded entries indicate that the solution-weight fell outside of the guidelines for a successful PCT result. 
Immobilization Technology Section

WSRC-TR-2001-00131

Savannah River Technology Center

Rev. 0

Westinghouse Savannah River Company

Table E3: Composition of PCT Leachate Solutions for Glasses from Nominally Washed Sludge (continued)

\begin{tabular}{|c|c|c|c|c|c|c|c|c|c|c|c|c|c|c|c|}
\hline & & & & \multicolumn{4}{|c|}{ Concentrations in ppm } & \multicolumn{4}{|c|}{ Concentrations in ppm } & \multirow{2}{*}{\multicolumn{4}{|c|}{ Common Logarithm of ppm Concentrations }} \\
\hline Glass & Lab & & & & (as re & orted) & & & r correc & for dilu & & & & & \\
\hline ID & ID & Blk & Seq & B & $\mathbf{L i}$ & $\mathbf{N a}$ & Si & B & $\mathbf{L i}$ & Na & Si & $\log [\mathbf{B}]$ & $\log [\mathrm{Li}]$ & $\log [\mathrm{Na}]$ & $\log [\mathrm{Si}]$ \\
\hline MB3N314clc & ga33 & 4 & 10 & 24.80 & 13.60 & 25.40 & 56.30 & 41.33 & 22.67 & 42.33 & 93.84 & 1.6163 & 1.3554 & 1.6267 & 1.9724 \\
\hline MB3N307clc & ga42 & 4 & 11 & 15.20 & 24.80 & 27.90 & 99.90 & 25.33 & 41.33 & 46.50 & 166.50 & 1.4037 & 1.6163 & 1.6675 & 2.2214 \\
\hline MB3N315clc & ga72 & 4 & 12 & 34.40 & 27.80 & 16.40 & 86.20 & 57.33 & 46.33 & 27.33 & 143.67 & 1.7584 & 1.6659 & 1.4367 & 2.1574 \\
\hline MB3N314q & ga13 & 4 & 13 & 28.20 & 15.00 & 27.50 & 54.80 & 47.00 & 25.00 & 45.83 & 91.34 & 1.6721 & 1.3979 & 1.6612 & 1.9606 \\
\hline MB3N315q & ga32 & 4 & 14 & 35.40 & 28.80 & 15.60 & 90.20 & 59.00 & 48.00 & 26.00 & 150.34 & 1.7709 & 1.6813 & 1.4150 & 2.1771 \\
\hline MB3N320q & ga34 & 4 & 15 & 11.60 & 22.10 & 82.20 & 126.00 & 19.33 & 36.83 & 137.00 & 210.00 & 1.2863 & 1.5663 & 2.1367 & 2.3222 \\
\hline std & std-b4-3 & 4 & 16 & 18.90 & 10.80 & 79.30 & 48.40 & 18.90 & 10.80 & 79.30 & 48.40 & 1.2765 & 1.0334 & 1.8993 & 1.6848 \\
\hline std & std-b5-1 & 5 & 1 & 18.90 & 10.50 & 77.10 & 47.30 & 18.90 & 10.50 & 77.10 & 47.30 & 1.2765 & 1.0212 & 1.8871 & 1.6749 \\
\hline MB3N320q & ga61 & 5 & 2 & 10.70 & 21.00 & 78.20 & 119.00 & 17.83 & 35.00 & 130.34 & 198.34 & 1.2512 & 1.5441 & 2.1151 & 2.2974 \\
\hline MB3N315q & ga48 & 5 & 3 & 33.90 & 27.80 & 14.80 & 86.10 & 56.50 & 46.33 & 24.67 & 143.50 & 1.7521 & 1.6659 & 1.3921 & 2.1569 \\
\hline MB3N314q & ga38 & 5 & 4 & 28.20 & 14.80 & 27.30 & 54.70 & 47.00 & 24.67 & 45.50 & 91.17 & 1.6721 & 1.3921 & 1.6580 & 1.9598 \\
\hline MB3N165clc & ga81 & 5 & 5 & 12.80 & 15.40 & 65.90 & 88.60 & 21.33 & 25.67 & 109.84 & 147.67 & 1.3291 & 1.4094 & 2.0407 & 2.1693 \\
\hline MB3N314clc & ga77 & 5 & 6 & 24.50 & 13.10 & 25.50 & 53.60 & 40.83 & 21.83 & 42.50 & 89.34 & 1.6110 & 1.3391 & 1.6284 & 1.9510 \\
\hline MB3N320clc & ga40 & 5 & 7 & 10.90 & 22.90 & 76.40 & 127.00 & 18.17 & 38.17 & 127.34 & 211.67 & 1.2593 & 1.5817 & 2.1050 & 2.3257 \\
\hline MB3N307q & ga05 & 5 & 8 & 15.60 & 31.80 & 31.30 & 127.00 & 26.00 & 53.00 & 52.17 & 211.67 & 1.4150 & 1.7243 & 1.7174 & 2.3257 \\
\hline std & std-b5-2 & 5 & 9 & 18.80 & 10.70 & 78.70 & 47.30 & 18.80 & 10.70 & 78.70 & 47.30 & 1.2742 & 1.0294 & 1.8960 & 1.6749 \\
\hline MB3N165q & ga70 & 5 & 10 & 13.50 & 15.70 & 72.80 & 90.80 & 22.50 & 26.17 & 121.34 & 151.34 & 1.3522 & 1.4178 & 2.0840 & 2.1799 \\
\hline ARM & ga31 & 5 & 11 & 10.80 & 9.31 & 22.20 & 36.00 & 18.00 & 15.52 & 37.00 & 60.00 & 1.2553 & 1.1908 & 1.5682 & 1.7782 \\
\hline MB3N200q & ga35 & 5 & 12 & 12.40 & 8.94 & 43.50 & 69.90 & 20.67 & 14.90 & 72.50 & 116.50 & 1.3153 & 1.1732 & 1.8603 & 2.0663 \\
\hline MB3N307clc & ga82 & 5 & 13 & 14.80 & 24.30 & 27.40 & 101.00 & 24.67 & 40.50 & 45.67 & 168.34 & 1.3921 & 1.6075 & 1.6596 & 2.2262 \\
\hline MB3N315clc & ga04 & 5 & 14 & 34.40 & 27.40 & 16.30 & 85.90 & 57.33 & 45.67 & 27.17 & 143.17 & 1.7584 & 1.6596 & 1.4340 & 2.1559 \\
\hline MB3N200clc & ga43 & 5 & 15 & 11.90 & 8.69 & 41.30 & 68.60 & 19.83 & 14.48 & 68.83 & 114.34 & 1.2974 & 1.1609 & 1.8378 & 2.0582 \\
\hline std & std-b5-3 & 5 & 16 & 19.00 & 10.80 & 79.10 & 47.70 & 19.00 & 10.80 & 79.10 & 47.70 & 1.2788 & 1.0334 & 1.8982 & 1.6785 \\
\hline std & std-b6-1 & 6 & 1 & 19.10 & 11.20 & 81.30 & 49.70 & 19.10 & 11.20 & 81.30 & 49.70 & 1.2810 & 1.0492 & 1.9101 & 1.6964 \\
\hline MB3N320q & ga52 & 6 & 2 & 10.80 & 21.00 & 77.60 & 119.00 & 18.00 & 35.00 & 129.34 & 198.34 & 1.2553 & 1.5441 & 2.1117 & 2.2974 \\
\hline MB3N307clc & ga03 & 6 & 3 & 15.50 & 25.10 & 28.20 & 102.00 & 25.83 & 41.83 & 47.00 & 170.00 & 1.4122 & 1.6215 & 1.6721 & 2.2305 \\
\hline ARM & ga02 & 6 & 4 & 11.30 & 9.91 & 23.20 & 39.00 & 18.83 & 16.52 & 38.67 & 65.00 & 1.2749 & 1.2179 & 1.5873 & 1.8129 \\
\hline MB3N200clc & ga44 & 6 & 5 & 12.70 & 9.26 & 43.30 & 74.20 & 21.17 & 15.43 & 72.17 & 123.67 & 1.3257 & 1.1885 & 1.8583 & 2.0923 \\
\hline MB3N314clc & ga01 & 6 & 6 & 24.50 & 13.40 & 27.20 & 56.50 & 40.83 & 22.33 & 45.33 & 94.17 & 1.6110 & 1.3490 & 1.6564 & 1.9739 \\
\hline MB3N165clc & ga30 & 6 & 7 & 12.10 & 15.30 & 64.80 & 86.20 & 20.17 & 25.50 & 108.00 & 143.67 & 1.3046 & 1.4065 & 2.0334 & 2.1574 \\
\hline MB3N320clc & ga73 & 6 & 8 & 10.50 & 22.70 & 75.10 & 122.00 & 17.50 & 37.83 & 125.17 & 203.34 & 1.2430 & 1.5779 & 2.0975 & 2.3082 \\
\hline std & std-b6-2 & 6 & 9 & 18.70 & 10.90 & 79.70 & 48.70 & 18.70 & 10.90 & 79.70 & 48.70 & 1.2718 & 1.0374 & 1.9015 & 1.6875 \\
\hline MB3N315clc & ga51 & 6 & 10 & 34.30 & 28.50 & 17.10 & 89.10 & 57.17 & 47.50 & 28.50 & 148.50 & 1.7572 & 1.6767 & 1.4549 & 2.1717 \\
\hline MB3N200q & ga68 & 6 & 11 & 13.40 & 9.80 & 47.40 & 75.90 & 22.33 & 16.33 & 79.00 & 126.50 & 1.3490 & 1.2131 & 1.8976 & 2.1021 \\
\hline blank & ga09 & 6 & 12 & $<0.150$ & $<0.040$ & 0.46 & $<0.790$ & 0.13 & 0.03 & 0.77 & 0.66 & -0.9031 & -1.4771 & -0.1116 & -0.1816 \\
\hline MB3N315q & ga19 & 6 & 13 & 33.90 & 28.20 & 15.10 & 87.00 & 56.50 & 47.00 & 25.17 & 145.00 & 1.7521 & 1.6721 & 1.4008 & 2.1614 \\
\hline MB3N314q & ga46 & 6 & 14 & 28.00 & 15.00 & 27.40 & 55.80 & 46.67 & 25.00 & 45.67 & 93.00 & 1.6690 & 1.3979 & 1.6596 & 1.9685 \\
\hline MB3N307q & ga07 & 6 & 15 & 16.40 & 33.10 & 33.00 & 125.00 & 27.33 & 55.17 & 55.00 & 208.34 & 1.4367 & 1.7417 & 1.7404 & 2.3188 \\
\hline MB3N165q & ga85 & 6 & 16 & 13.20 & 15.80 & 74.20 & 89.80 & 22.00 & 26.33 & 123.67 & 149.67 & 1.3424 & 1.4205 & 2.0923 & 2.1751 \\
\hline std & std-b6-3 & 6 & 17 & 18.90 & 11.00 & 80.10 & 49.50 & 18.90 & 11.00 & 80.10 & 49.50 & 1.2765 & 1.0414 & 1.9036 & 1.6946 \\
\hline
\end{tabular}

Notes:

(1). Values that were below detection (indicated by a "<") were converted to $1 / 2$ the detection limit.

(3) The shaded entries indicate that the solution-weight fell outside of the guidelines for a successful PCT result. 
Immobilization Technology Section

WSRC-TR-2001-00131

Savannah River Technology Center

Rev. 0

Westinghouse Savannah River Company

Table E4: Composition of PCT Leachate Solutions for Glasses from Underwashed Sludge

\begin{tabular}{|c|c|c|c|c|c|c|c|c|c|c|c|c|c|c|c|}
\hline \multirow{3}{*}{\begin{tabular}{|c|} 
Glass \\
ID \\
\end{tabular}} & \multirow{3}{*}{$\begin{array}{c}\text { Lab } \\
\text { ID }\end{array}$} & \multirow{3}{*}{ Blk } & \multirow{3}{*}{ Seq } & \multicolumn{4}{|c|}{ Concentrations in ppm } & \multicolumn{4}{|c|}{ Concentrations in ppm } & \multirow{2}{*}{\multicolumn{4}{|c|}{ Common Logarithm of ppm Concentrations }} \\
\hline & & & & & (as re & orted) & & & r correc & for dilu & & & & & \\
\hline & & & & B & $\mathbf{L i}$ & $\mathrm{Na}$ & $\mathbf{S i}$ & B & $\mathbf{L i}$ & $\mathrm{Na}$ & $\mathbf{S i}$ & $\log [B]$ & $\log [\mathrm{Li}]$ & $\log [\mathrm{Na}]$ & $\log [\mathrm{Si}]$ \\
\hline std & std-b1-1 & 1 & $\frac{1}{1}$ & 18.90 & 9.45 & 78.60 & 47.50 & 18.90 & 9.45 & 78.60 & 47.50 & 1.2765 & 0.9754 & 1.8954 & 1.6767 \\
\hline MB3U200q & jp33 & 1 & 2 & 14.10 & 8.93 & 53.90 & 76.90 & 23.50 & 14.88 & 89.84 & 128.17 & 1.3711 & 1.1727 & 1.9534 & 2.1078 \\
\hline EA & jp83 & 1 & 3 & 34.40 & 10.80 & 97.40 & 51.80 & 573.33 & 180.00 & 1623.34 & 863.34 & 2.7584 & 2.2553 & 3.2104 & 2.9362 \\
\hline MB3U165clc & jp79 & 1 & 4 & 13.80 & 15.50 & 76.50 & 95.50 & 23.00 & 25.83 & 127.50 & 159.17 & 1.3617 & 1.4122 & 2.1055 & 2.2019 \\
\hline MB3U165q & jp73 & 1 & 5 & 14.40 & 15.40 & 84.60 & 97.70 & 24.00 & 25.67 & 141.00 & 162.84 & 1.3802 & 1.4094 & 2.1492 & 2.2118 \\
\hline MB3U303clc & jp78 & 1 & 6 & 20.40 & 17.70 & 12.20 & 68.60 & 34.00 & 29.50 & 20.33 & 114.34 & 1.5315 & 1.4698 & 1.3082 & 2.0582 \\
\hline MB3U313q & jp14 & 1 & 7 & 11.20 & 34.60 & 80.20 & 147.00 & 18.67 & 57.67 & 133.67 & 245.00 & 1.2711 & 1.7609 & 2.1260 & 2.3892 \\
\hline MB3U200clc & jp68 & 1 & 8 & 12.80 & 8.19 & 48.20 & 73.70 & 21.33 & 13.65 & 80.33 & 122.84 & 1.3291 & 1.1351 & 1.9049 & 2.0893 \\
\hline MB3U314q & jp43 & 1 & 9 & 31.30 & 15.20 & 34.90 & 57.80 & 52.17 & 25.33 & 58.17 & 96.34 & 1.7174 & 1.4037 & 1.7647 & 1.9838 \\
\hline std & std-b1-2 & 1 & 10 & 18.90 & 9.49 & 79.40 & 48.30 & 18.90 & 9.49 & 79.40 & 48.30 & 1.2765 & 0.9773 & 1.8998 & 1.6839 \\
\hline MB3U313clc & jp74 & 1 & 11 & 14.90 & 62.40 & 91.40 & 218.00 & 24.83 & 104.00 & 152.34 & 363.34 & 1.3950 & 2.0170 & 2.1828 & 2.5603 \\
\hline ARM & jp01 & 1 & 12 & 11.30 & 9.09 & 24.30 & 39.10 & 18.83 & 15.15 & 40.50 & 65.17 & 1.2749 & 1.1804 & 1.6075 & 1.8140 \\
\hline MB3U304clc & jp42 & 1 & 13 & 9.13 & 14.90 & 140.00 & 109.00 & 15.22 & 24.83 & 233.34 & 181.67 & 1.1823 & 1.3950 & 2.3680 & 2.2593 \\
\hline MB3U304q & jp24 & 1 & 14 & 9.83 & 15.00 & 160.00 & 121.00 & 16.38 & 25.00 & 266.67 & 201.67 & 1.2144 & 1.3979 & 2.4260 & 2.3046 \\
\hline MB3U303q & jp50 & 1 & 15 & 22.60 & 19.80 & 11.60 & 71.30 & 37.67 & 33.00 & 19.33 & 118.84 & 1.5760 & 1.5185 & 1.2863 & 2.0749 \\
\hline blank & jp52 & 1 & 16 & $<0.150$ & 0.37 & 0.61 & $<0.790$ & 0.13 & 0.62 & 1.02 & 0.66 & -0.9031 & -0.2111 & 0.0065 & -0.1816 \\
\hline MB3U314clc & jp69 & 1 & 17 & 28.30 & 13.50 & 32.80 & 57.20 & 47.17 & 22.50 & 54.67 & 95.34 & 1.6736 & 1.3522 & 1.7377 & 1.9793 \\
\hline std & std-b1-3 & 1 & 18 & 18.90 & 9.50 & 79.90 & 47.90 & 18.90 & 9.50 & 79.90 & 47.90 & 1.2765 & 0.9777 & 1.9025 & 1.6803 \\
\hline std & std-b2-1 & 2 & 1 & 18.80 & 9.20 & 77.60 & 47.80 & 18.80 & 9.20 & 77.60 & 47.80 & 1.2742 & 0.9638 & 1.8899 & 1.6794 \\
\hline blank & jp36 & 2 & 2 & $<0.150$ & 0.35 & 0.59 & $<0.790$ & 0.13 & 0.59 & 0.99 & 0.66 & -0.9031 & -0.2316 & -0.0044 & -0.1816 \\
\hline MB3U304q & jp56 & 2 & 3 & 9.10 & 13.90 & 146.00 & 128.00 & 15.17 & 23.17 & 243.34 & 213.34 & 1.1809 & 1.3649 & 2.3862 & 2.3291 \\
\hline ARM & jp11 & 2 & 4 & 9.97 & 8.15 & 21.90 & 36.30 & 16.62 & 13.58 & 36.50 & 60.50 & 1.2206 & 1.1330 & 1.5623 & 1.7818 \\
\hline MB3U303q & jp19 & 2 & 5 & 22.30 & 19.00 & 11.30 & 69.80 & 37.17 & 31.67 & 18.83 & 116.34 & 1.5702 & 1.5006 & 1.2749 & 2.0657 \\
\hline MB3U200q & jp75 & 2 & 6 & 13.70 & 8.53 & 51.70 & 73.50 & 22.83 & 14.22 & 86.17 & 122.50 & 1.3586 & 1.1528 & 1.9353 & 2.0881 \\
\hline MB3U314q & jp71 & 2 & 7 & 30.10 & 14.30 & 32.80 & 55.80 & 50.17 & 23.83 & 54.67 & 93.00 & 1.7004 & 1.3772 & 1.7377 & 1.9685 \\
\hline MB3U313q & jp06 & 2 & 8 & 10.70 & 32.70 & 76.40 & 158.00 & 17.83 & 54.50 & 127.34 & 263.34 & 1.2512 & 1.7364 & 2.1050 & 2.4205 \\
\hline MB3U304clc & jp62 & 2 & 9 & 8.46 & 13.80 & 130.00 & 120.00 & 14.10 & 23.00 & 216.67 & 200.00 & 1.1492 & 1.3617 & 2.3358 & 2.3010 \\
\hline std & std-b2-2 & 2 & 10 & 18.10 & 9.14 & 76.80 & 46.70 & 18.10 & 9.14 & 76.80 & 46.70 & 1.2577 & 0.9609 & 1.8854 & 1.6693 \\
\hline MB3U165q & jp55 & 2 & 11 & 13.80 & 14.60 & 80.00 & 94.30 & 23.00 & 24.33 & 133.34 & 157.17 & 1.3617 & 1.3862 & 2.1249 & 2.1964 \\
\hline MB3U313clc & jp84 & 2 & 12 & 14.40 & 59.80 & 88.30 & 257.00 & 24.00 & 99.67 & 147.17 & 428.34 & 1.3802 & 1.9986 & 2.1678 & 2.6318 \\
\hline MB3U200clc & jp41 & 2 & 13 & 12.60 & 8.10 & 47.00 & 71.90 & 21.00 & 13.50 & 78.33 & 119.84 & 1.3222 & 1.1303 & 1.8940 & 2.0786 \\
\hline MB3U165clc & jp17 & 2 & 14 & 13.30 & 14.80 & 73.90 & 93.00 & 22.17 & 24.67 & 123.17 & 155.00 & 1.3457 & 1.3921 & 2.0905 & 2.1903 \\
\hline MB3U314clc & jp54 & 2 & 15 & 28.50 & 13.30 & 32.80 & 58.00 & 47.50 & 22.17 & 54.67 & 96.67 & 1.6767 & 1.3457 & 1.7377 & 1.9853 \\
\hline EA & jp27 & 2 & 16 & 37.10 & 11.20 & 102.00 & 55.00 & 618.33 & 186.67 & 1700.00 & 916.67 & 2.7912 & 2.2711 & 3.2305 & 2.9622 \\
\hline MB3U303clc & jp13 & 2 & 17 & 20.30 & 17.30 & 11.90 & 69.60 & 33.83 & 28.83 & 19.83 & 116.00 & 1.5294 & 1.4599 & 1.2974 & 2.0645 \\
\hline std & std-b2-3 & 2 & 18 & 18.50 & 9.18 & 76.20 & 47.60 & 18.50 & 9.18 & 76.20 & 47.60 & 1.2672 & 0.9628 & 1.8820 & 1.6776 \\
\hline std & std-b3-1 & 3 & 1 & 18.80 & 9.28 & 78.30 & 47.80 & 18.80 & 9.28 & 78.30 & 47.80 & 1.2742 & 0.9675 & 1.8938 & 1.6794 \\
\hline MB3U200q & jp46 & 3 & 2 & 14.20 & 8.90 & 53.40 & 77.10 & 23.67 & 14.83 & 89.00 & 128.50 & 1.3741 & 1.1712 & 1.9494 & 2.1089 \\
\hline ARM & jp67 & 3 & 3 & 10.50 & 8.51 & 23.00 & 38.00 & 17.50 & 14.18 & 38.33 & 63.33 & 1.2430 & 1.1518 & 1.5836 & 1.8016 \\
\hline MB3U314clc & jp22 & 3 & 4 & 28.40 & 13.40 & 33.00 & 57.00 & 47.33 & 22.33 & 55.00 & 95.00 & 1.6752 & 1.3490 & 1.7404 & 1.9777 \\
\hline MB3U313q & jp31 & 3 & 5 & 10.70 & 33.50 & 77.60 & 153.00 & 17.83 & 55.83 & 129.34 & 255.01 & 1.2512 & 1.7469 & 2.1117 & 2.4065 \\
\hline MB3U303clc & jp10 & 3 & 6 & 19.50 & 16.90 & 11.80 & 67.50 & 32.50 & 28.17 & 19.67 & 112.50 & 1.5119 & 1.4497 & 1.2937 & 2.0512 \\
\hline MB3U314q & jp08 & 3 & 7 & 31.40 & 15.10 & 33.80 & 58.10 & 52.33 & 25.17 & 56.33 & 96.84 & 1.7188 & 1.4008 & 1.7508 & 1.9860 \\
\hline MB3U303q & jp59 & 3 & 8 & 22.00 & 19.00 & 11.20 & 69.10 & 36.67 & 31.67 & 18.67 & 115.17 & 1.5643 & 1.5006 & 1.2711 & 2.0613 \\
\hline MB3U304q & jp07 & 3 & 9 & 10.00 & 15.40 & 160.00 & 128.00 & 16.67 & 25.67 & 266.67 & 213.34 & 1.2219 & 1.4094 & 2.4260 & 2.3291 \\
\hline std & std-b3-2 & 3 & 10 & 18.70 & 9.31 & 78.20 & 47.70 & 18.70 & 9.31 & 78.20 & 47.70 & 1.2718 & 0.9690 & 1.8932 & 1.6785 \\
\hline MB3U200clc & jp15 & 3 & 11 & 12.90 & 8.29 & 48.30 & 72.50 & 21.50 & 13.82 & 80.50 & 120.84 & 1.3324 & 1.1404 & 1.9058 & 2.0822 \\
\hline MB3U165clc & jp48 & 3 & 12 & 13.10 & 14.80 & 73.80 & 92.70 & 21.83 & 24.67 & 123.00 & 154.50 & 1.3391 & 1.3921 & 2.0899 & 2.1889 \\
\hline MB3U165q & jp65 & 3 & 13 & 14.10 & 15.10 & 82.40 & 97.00 & 23.50 & 25.17 & 137.34 & 161.67 & 1.3711 & 1.4008 & 2.1378 & 2.2086 \\
\hline MB3U304clc & jp16 & 3 & 14 & 9.04 & 14.80 & 138.00 & 112.00 & 15.07 & 24.67 & 230.00 & 186.67 & 1.1780 & 1.3921 & 2.3617 & 2.2711 \\
\hline EA & jp64 & 3 & 15 & 34.70 & 11.00 & 97.80 & 52.40 & 578.33 & 183.33 & 1630.00 & 873.34 & 2.7622 & 2.2632 & 3.2122 & 2.9412 \\
\hline MB3U313clc & jp28 & 3 & 16 & 15.00 & 62.90 & 92.70 & 274.00 & 25.00 & 104.84 & 154.50 & 456.68 & 1.3979 & 2.0205 & 2.1889 & 2.6596 \\
\hline std & std-b3-3 & 3 & 17 & 18.50 & 9.25 & 78.30 & 46.90 & 18.50 & 9.25 & 78.30 & 46.90 & 1.2672 & 0.9661 & 1.8938 & 1.6712 \\
\hline std & std-b4-1 & 4 & 1 & 18.60 & 9.29 & 79.80 & 46.70 & 18.60 & 9.29 & 79.80 & 46.70 & 1.2695 & 0.9680 & 1.9020 & 1.6693 \\
\hline MB3U320clc & jp21 & 4 & 2 & 10.30 & 20.60 & 76.90 & 121.00 & 17.17 & 34.33 & 128.17 & 201.67 & 1.2347 & 1.5357 & 2.1078 & 2.3046 \\
\hline MB3U322clc & jp63 & 4 & 3 & 5.63 & 7.10 & 37.20 & 73.10 & 9.38 & 11.83 & 62.00 & 121.84 & 0.9724 & 1.0731 & 1.7924 & 2.0858 \\
\hline MB3U324q & jp03 & 4 & 4 & 20.40 & 19.40 & 32.40 & 89.60 & 34.00 & 32.33 & 54.00 & 149.34 & 1.5315 & 1.5097 & 1.7324 & 2.1742 \\
\hline MB3U307q & jp38 & 4 & 5 & 16.90 & 33.60 & 38.60 & 118.00 & 28.17 & 56.00 & 64.33 & 196.67 & 1.4497 & 1.7482 & 1.8084 & 2.2937 \\
\hline MB3U326q & jp47 & 4 & 6 & 12.30 & 20.70 & 83.90 & 120.00 & 20.50 & 34.50 & 139.84 & 200.00 & 1.3118 & 1.5378 & 2.1456 & 2.3010 \\
\hline MB3U326clc & jp45 & 4 & 7 & 12.10 & 21.60 & 77.10 & 118.00 & 20.17 & 36.00 & 128.50 & 196.67 & 1.3046 & 1.5563 & 2.1089 & 2.2937 \\
\hline MB3U320q & jp70 & 4 & 8 & 11.10 & 20.10 & 86.70 & 112.00 & 18.50 & 33.50 & 144.50 & 186.67 & 1.2672 & 1.5251 & 2.1599 & 2.2711 \\
\hline MB3U323q & jp37 & 4 & 9 & 17.00 & 10.00 & 36.40 & 70.50 & 28.33 & 16.67 & 60.67 & 117.50 & 1.4523 & 1.2219 & 1.7830 & 2.0700 \\
\hline std & std-b4-2 & 4 & 10 & 18.50 & 9.26 & 79.30 & 46.50 & 18.50 & 9.26 & 79.30 & 46.50 & 1.2672 & 0.9666 & 1.8993 & 1.6675 \\
\hline MB3U307clc & jp60 & 4 & 11 & 18.70 & 28.40 & 38.40 & 108.00 & 31.17 & 47.33 & 64.00 & 180.00 & 1.4937 & 1.6752 & 1.8062 & 2.2553 \\
\hline
\end{tabular}

Notes:

(1). Values that were below detection (indicated by a "<") were converted to $1 / 2$ the detection limit.

(4) The shaded entries indicate that the solution-weight fell outside of the guidelines for a successful PCT result. 
Immobilization Technology Section

WSRC-TR-2001-00131

Savannah River Technology Center

Rev. 0

Westinghouse Savannah River Company

Table E4: Composition of PCT Leachate Solutions for Glasses from Underwashed Sludge

\begin{tabular}{|c|c|c|c|c|c|c|c|c|c|c|c|c|c|c|c|}
\hline & & & & \multicolumn{4}{|c|}{ Concentrations in ppm } & \multicolumn{4}{|c|}{ Concentrations in ppm } & \multirow{2}{*}{\multicolumn{4}{|c|}{ Common Logarithm of ppm Concentrations }} \\
\hline Glass & Lab & & & & (as re & orted) & & & correc & for dilu & & & & & \\
\hline ID & ID & Blk & Seq & B & $\mathbf{L i}$ & $\mathrm{Na}$ & $\mathbf{S i}$ & B & $\mathbf{L i}$ & $\mathrm{Na}$ & $\mathrm{Si}$ & $\log [B]$ & $\log [\mathrm{Li}]$ & $\log [\mathrm{Na}]$ & $\log [\mathrm{Si}]$ \\
\hline MB3U324clc & jp80 & 4 & 12 & 17.70 & 16.70 & 30.30 & 83.50 & 29.50 & 27.83 & 50.50 & 139.17 & 1.4698 & 1.4446 & 1.7033 & 2.1435 \\
\hline MB3U322q & jp39 & 4 & 13 & 5.70 & 7.12 & 38.90 & 73.30 & 9.50 & 11.87 & 64.83 & 122.17 & 0.9777 & 1.0743 & 1.8118 & 2.0870 \\
\hline MB3U323clc & jp66 & 4 & 14 & 14.60 & 8.61 & 32.40 & 67.50 & 24.33 & 14.35 & 54.00 & 112.50 & 1.3862 & 1.1569 & 1.7324 & 2.0512 \\
\hline MB3U315q & jp23 & 4 & 15 & 40.10 & 32.50 & 20.60 & 96.30 & 66.83 & 54.17 & 34.33 & 160.50 & 1.8250 & 1.7337 & 1.5357 & 2.2055 \\
\hline MB3U315clc & jp44 & 4 & 16 & 40.20 & 31.40 & 22.20 & 95.70 & 67.00 & 52.33 & 37.00 & 159.50 & 1.8261 & 1.7188 & 1.5682 & 2.2028 \\
\hline std & std-b4-3 & 4 & 17 & 19.00 & 9.35 & 79.30 & 47.60 & 19.00 & 9.35 & 79.30 & 47.60 & 1.2788 & 0.9708 & 1.8993 & 1.6776 \\
\hline std & std-b5-1 & 5 & 1 & 19.50 & 9.52 & 79.60 & 48.80 & 19.50 & 9.52 & 79.60 & 48.80 & 1.2900 & 0.9786 & 1.9009 & 1.6884 \\
\hline MB3U323clc & jp29 & 5 & 2 & 15.50 & 8.59 & 32.30 & 68.50 & 25.83 & 14.32 & 53.83 & 114.17 & 1.4122 & 1.1559 & 1.7311 & 2.0575 \\
\hline MB3U326q & jp02 & 5 & 3 & 12.20 & 19.50 & 79.20 & 119.00 & 20.33 & 32.50 & 132.00 & 198.34 & 1.3082 & 1.5119 & 2.1206 & 2.2974 \\
\hline MB3U322clc & jp18 & 5 & 4 & 5.88 & 7.08 & 36.00 & 75.20 & 9.80 & 11.80 & 60.00 & 125.34 & 0.9912 & 1.0719 & 1.7782 & 2.0981 \\
\hline MB3U322q & jp30 & 5 & 5 & 5.74 & 6.91 & 35.20 & 73.10 & 9.57 & 11.52 & 58.67 & 121.84 & 0.9808 & 1.0613 & 1.7684 & 2.0858 \\
\hline MB3U324q & jp77 & 5 & 6 & 21.50 & 18.90 & 30.80 & 91.30 & 35.83 & 31.50 & 51.33 & 152.17 & 1.5543 & 1.4983 & 1.7104 & 2.1823 \\
\hline MB3U320q & jp32 & 5 & 7 & 10.90 & 18.50 & 77.90 & 116.00 & 18.17 & 30.83 & 129.84 & 193.34 & 1.2593 & 1.4890 & 2.1134 & 2.2863 \\
\hline MB3U323q & jp49 & 5 & 8 & 16.90 & 9.47 & 34.30 & 71.50 & 28.17 & 15.78 & 57.17 & 119.17 & 1.4497 & 1.1982 & 1.7572 & 2.0762 \\
\hline MB3U315q & jp86 & 5 & 9 & 28.10 & 20.80 & 13.70 & 63.80 & 46.83 & 34.67 & 22.83 & 106.34 & 1.6706 & 1.5399 & 1.3586 & 2.0267 \\
\hline std & std-b5-2 & 5 & 10 & 19.20 & 9.34 & 76.90 & 48.70 & 19.20 & 9.34 & 76.90 & 48.70 & 1.2833 & 0.9703 & 1.8859 & 1.6875 \\
\hline MB3U315clc & jp12 & 5 & 11 & 39.90 & 30.60 & 22.40 & 92.70 & 66.50 & 51.00 & 37.33 & 154.50 & 1.8228 & 1.7076 & 1.5721 & 2.1889 \\
\hline MB3U324clc & jp57 & 5 & 12 & 19.30 & 17.00 & 30.40 & 87.70 & 32.17 & 28.33 & 50.67 & 146.17 & 1.5074 & 1.4523 & 1.7047 & 2.1649 \\
\hline MB3U307clc & jp61 & 5 & 13 & 19.70 & 27.80 & 37.10 & 115.00 & 32.83 & 46.33 & 61.83 & 191.67 & 1.5163 & 1.6659 & 1.7912 & 2.2826 \\
\hline MB3U320clc & jp76 & 5 & 14 & 10.60 & 20.20 & 73.90 & 122.00 & 17.67 & 33.67 & 123.17 & 203.34 & 1.2472 & 1.5272 & 2.0905 & 2.3082 \\
\hline MB3U307q & jp51 & 5 & 15 & 17.60 & 33.00 & 37.30 & 143.00 & 29.33 & 55.00 & 62.17 & 238.34 & 1.4674 & 1.7404 & 1.7936 & 2.3772 \\
\hline MB3U326clc & jp26 & 5 & 16 & 12.80 & 21.40 & 75.40 & 127.00 & 21.33 & 35.67 & 125.67 & 211.67 & 1.3291 & 1.5523 & 2.0992 & 2.3257 \\
\hline std & std-b5-3 & 5 & 17 & 19.00 & 9.32 & 77.20 & 47.80 & 19.00 & 9.32 & 77.20 & 47.80 & 1.2788 & 0.9694 & 1.8876 & 1.6794 \\
\hline std & std-b6-1 & 6 & 1 & 18.80 & 9.33 & 80.10 & 45.20 & 18.80 & 9.33 & 80.10 & 45.20 & 1.2742 & 0.9699 & 1.9036 & 1.6551 \\
\hline MB3U315q & jp05 & 6 & 2 & 40.70 & 31.40 & 20.20 & 91.30 & 67.83 & 52.33 & 33.67 & 152.17 & 1.8315 & 1.7188 & 1.5272 & 2.1823 \\
\hline MB3U315clc & jp58 & 6 & 3 & 40.40 & 30.00 & 21.70 & 91.30 & 67.33 & 50.00 & 36.17 & 152.17 & 1.8282 & 1.6990 & 1.5583 & 2.1823 \\
\hline MB3U324clc & jp20 & 6 & 4 & 18.70 & 16.70 & 29.60 & 82.00 & 31.17 & 27.83 & 49.33 & 136.67 & 1.4937 & 1.4446 & 1.6931 & 2.1357 \\
\hline MB3U326q & jp09 & 6 & 5 & 12.50 & 20.40 & 82.00 & 118.00 & 20.83 & 34.00 & 136.67 & 196.67 & 1.3188 & 1.5315 & 2.1357 & 2.2937 \\
\hline MB3U326clc & jp34 & 6 & 6 & 12.60 & 21.20 & 75.00 & 120.00 & 21.00 & 35.33 & 125.00 & 200.00 & 1.3222 & 1.5482 & 2.0969 & 2.3010 \\
\hline MB3U307clc & jp82 & 6 & 7 & 19.90 & 28.40 & 37.70 & 114.00 & 33.17 & 47.33 & 62.83 & 190.00 & 1.5207 & 1.6752 & 1.7982 & 2.2788 \\
\hline MB3U323clc & jp85 & 6 & 8 & 16.20 & 9.00 & 33.50 & 68.20 & 27.00 & 15.00 & 55.83 & 113.67 & 1.4314 & 1.1761 & 1.7469 & 2.0556 \\
\hline MB3U307q & jp35 & 6 & 9 & 17.30 & 32.90 & 37.60 & 138.00 & 28.83 & 54.83 & 62.67 & 230.00 & 1.4599 & 1.7391 & 1.7970 & 2.3617 \\
\hline std & std-b6-2 & 6 & 10 & 19.30 & 9.49 & 79.20 & 46.30 & 19.30 & 9.49 & 79.20 & 46.30 & 1.2856 & 0.9773 & 1.8987 & 1.6656 \\
\hline MB3U320q & jp04 & 6 & 11 & 11.10 & 19.40 & 81.50 & 119.00 & 18.50 & 32.33 & 135.84 & 198.34 & 1.2672 & 1.5097 & 2.1330 & 2.2974 \\
\hline MB3U322clc & jp25 & 6 & 12 & 5.86 & 7.14 & 35.90 & 72.70 & 9.77 & 11.90 & 59.83 & 121.17 & 0.9898 & 1.0756 & 1.7770 & 2.0834 \\
\hline MB3U323q & jp72 & 6 & 13 & 17.30 & 9.74 & 35.30 & 68.80 & 28.83 & 16.23 & 58.83 & 114.67 & 1.4599 & 1.2104 & 1.7696 & 2.0594 \\
\hline MB3U320clc & jp53 & 6 & 14 & 10.40 & 20.20 & 75.10 & 119.00 & 17.33 & 33.67 & 125.17 & 198.34 & 1.2389 & 1.5272 & 2.0975 & 2.2974 \\
\hline MB3U322q & jp81 & 6 & 15 & 5.98 & 7.28 & 38.50 & 72.40 & 9.97 & 12.13 & 64.17 & 120.67 & 0.9986 & 1.0840 & 1.8073 & 2.0816 \\
\hline MB3U324q & jp40 & 6 & 16 & 21.40 & 19.10 & 31.90 & 87.90 & 35.67 & 31.83 & 53.17 & 146.50 & 1.5523 & 1.5029 & 1.7256 & 2.1658 \\
\hline std & std-b6-3 & 6 & 17 & 18.90 & 9.32 & 78.30 & 45.10 & 18.90 & 9.32 & 78.30 & 45.10 & 1.2765 & 0.9694 & 1.8938 & 1.6542 \\
\hline
\end{tabular}

Notes:

(1). Values that were below detection (indicated by a "<") were converted to $1 / 2$ the detection limit.

(5) The shaded entries indicate that the solution-weight fell outside of the guidelines for a successful PCT result. 
Westinghouse Savannah River Company

Exhibit E1. Plot of Oxide Concentrations (as wt\%'s) in Analytical Sequence for LM Prep
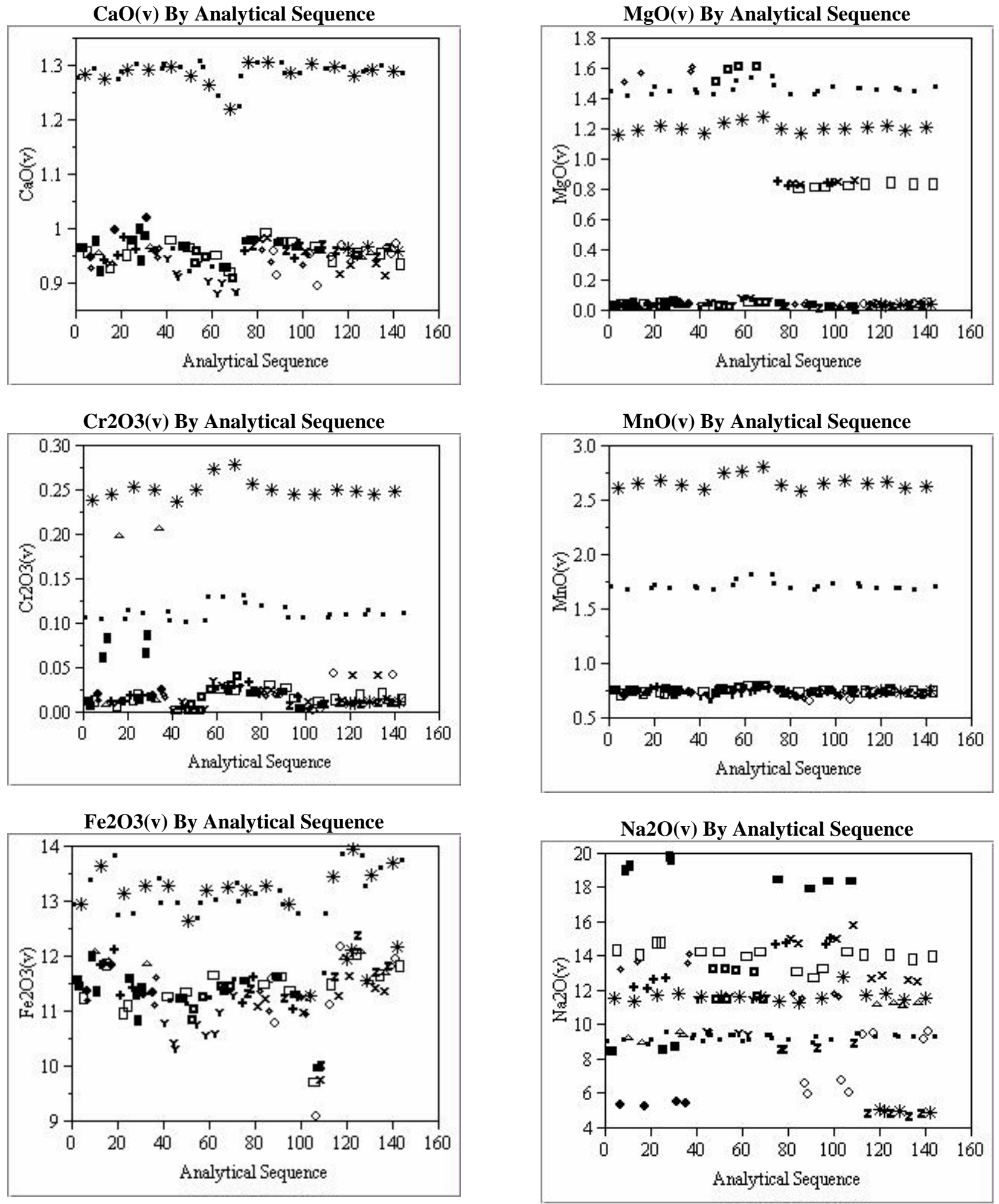
Exhibit E1. Plot of Oxide Concentrations (as wt\%'s) in Analytical Sequence for LM Prep (continued)

$\mathrm{NiO}(v)$ By Analytical Sequence

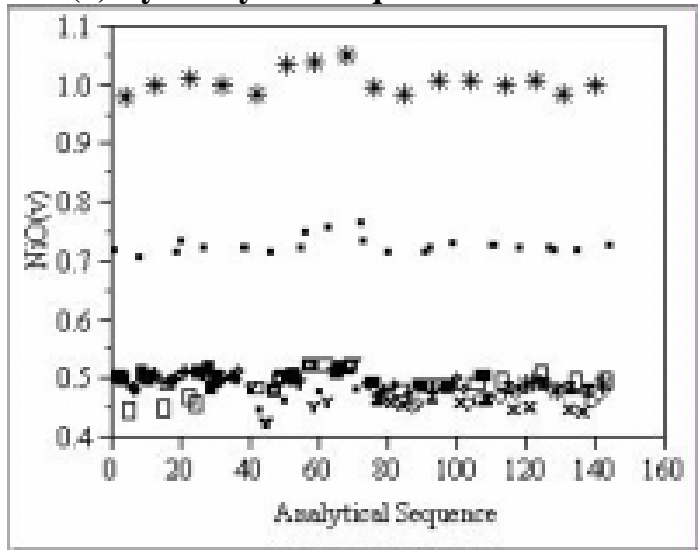

\section{SiO2(v) By Analytical Sequence}

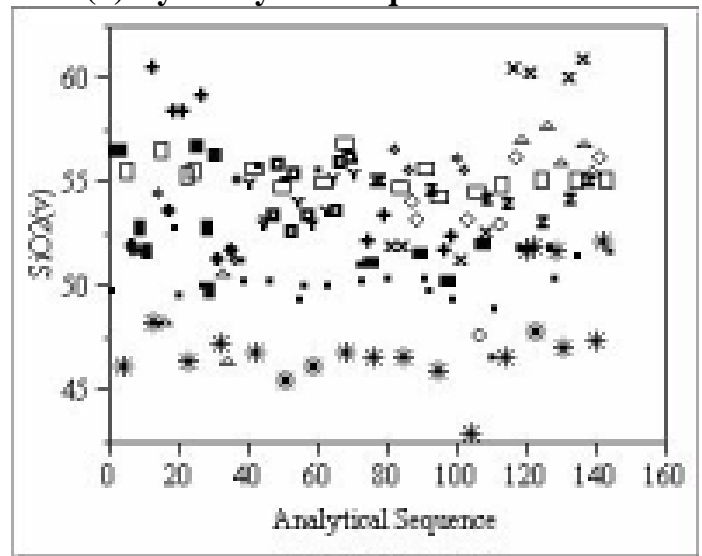

ZrO2(v) By Analytical Sequence

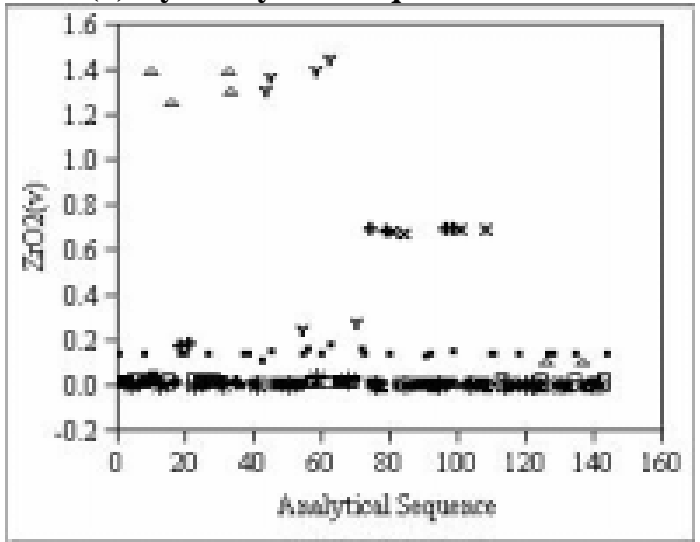


Exhibit E2. Plot of Oxide Concentrations (as wt\%'s) in Analytical Sequence for PF Prep

Al2O3 (v) By Analytical Sequence

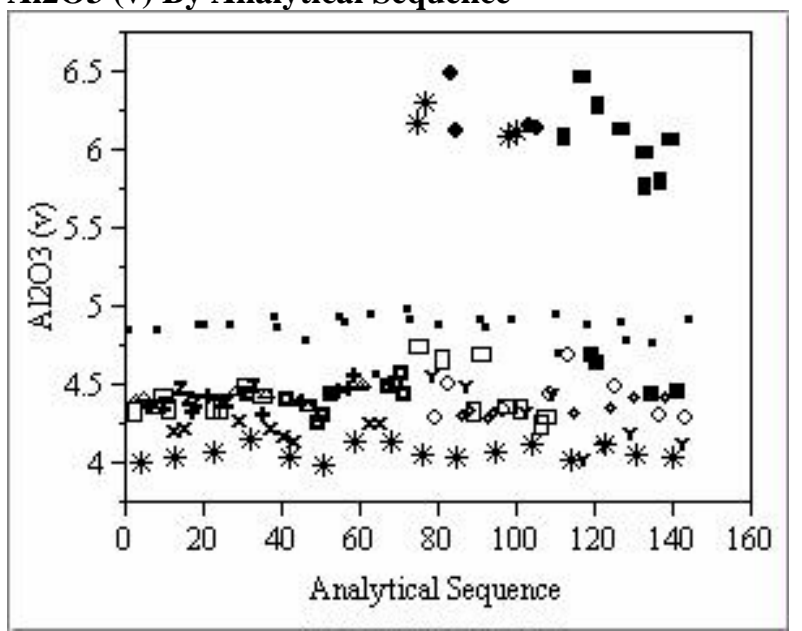

B2O3(v) By Analytical Sequence

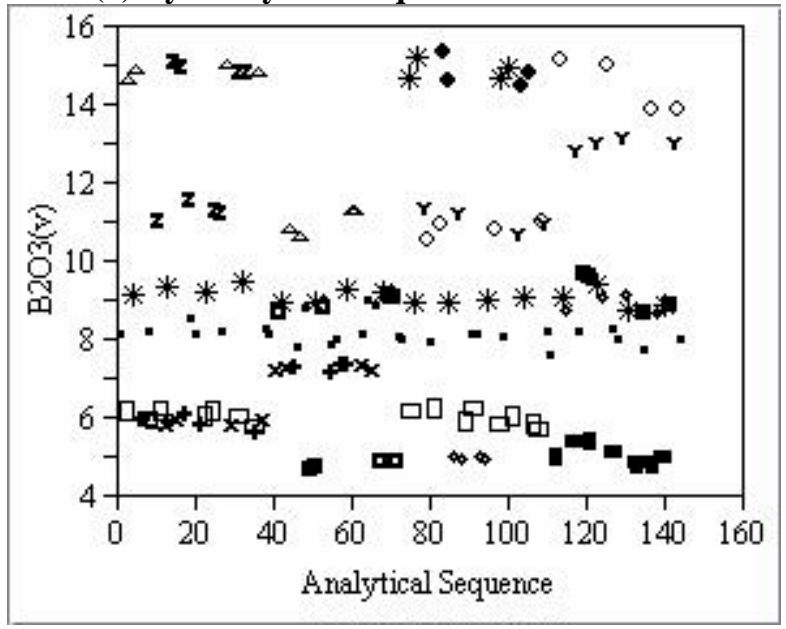

Li2O(v) By Analytical Sequence

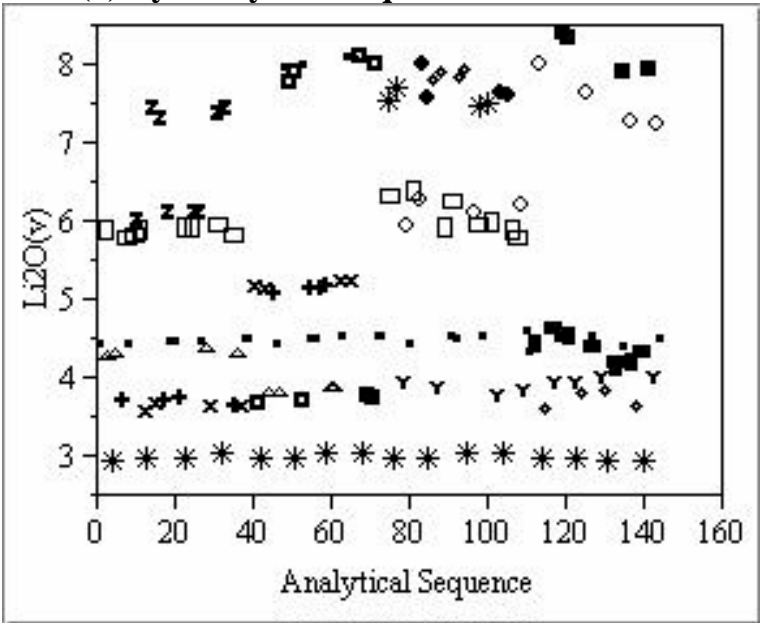

U3O8(v) By Analytical Sequence

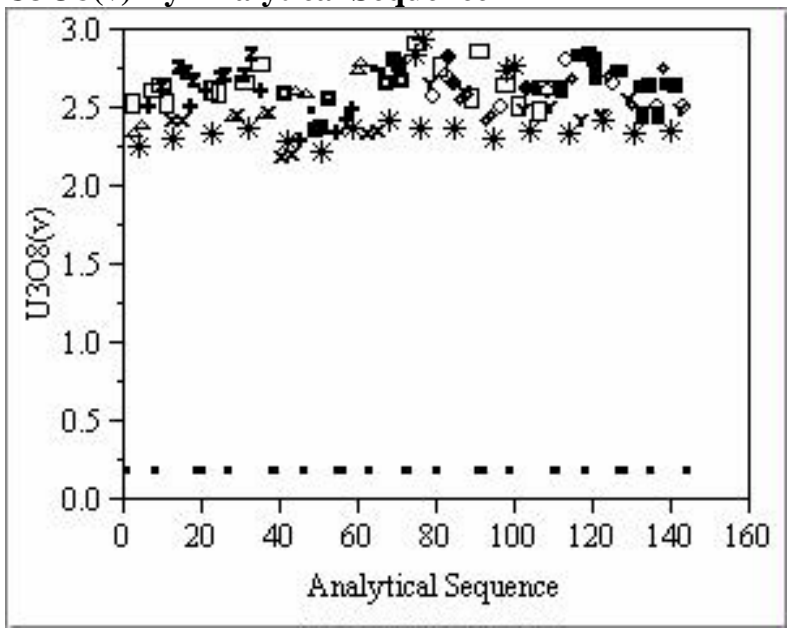


Exhibit E3. Measurements of Standards Prepared Using the Lithium Metaborate (LM) Method by Oxide by Analytical Block (Small Square - Batch 1 and Asterisk - Ustd)

Oneway Analysis of CaO(v) By Analytical Blks (lm)

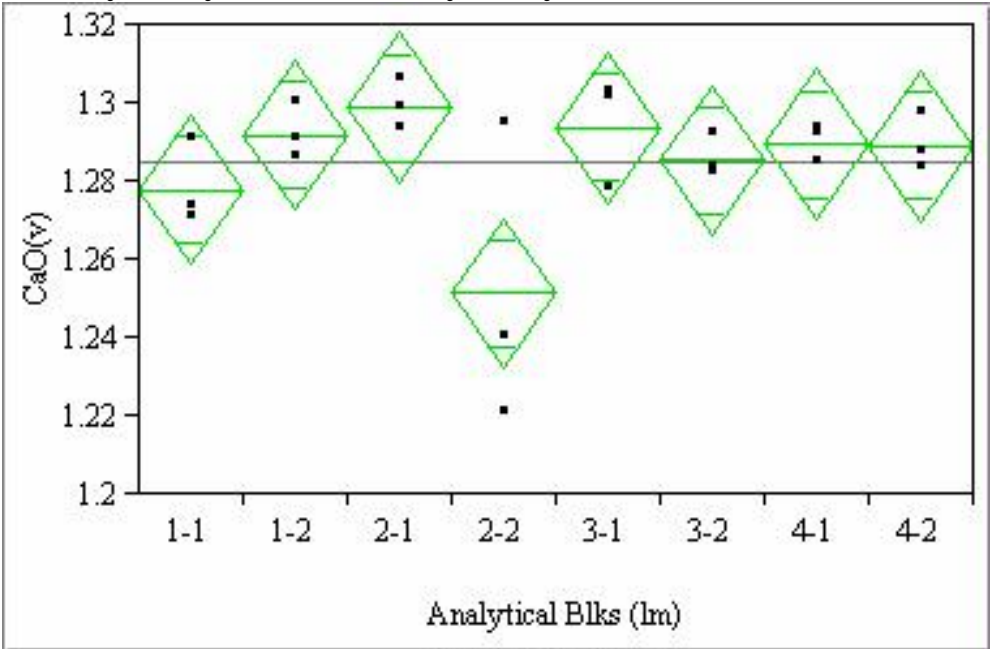

\section{Oneway Anova}

Adj Rsquare

0.538258

0.336246
Adj Rsquare

Root Mean Square Error $\quad 0.015735$

Observations (or Sum Wgts)

Analysis of Variance

Source DF Sum of Squares Mean Square F Ratio Prob > F

$\begin{array}{lrrrrr}\text { Analytical Blks (lm) } & 7 & 0.00461762 & 0.000660 & 2.6645 & 0.0495\end{array}$

0.000248

Means for Oneway Anova

Level Number Mean Std Error Lower 95\% Upper 95\%

$\begin{array}{llllll}1-1 & 3 & 1.27794 & 0.00908 & 1.2587 & 1.2972\end{array}$

$\begin{array}{llllll}1-2 & 3 & 1.29193 & 0.00908 & 1.2727 & 1.3112 \\ 2-1 & 3 & 1.29892 & 0.00908 & 1.2797 & 1.3182\end{array}$

$\begin{array}{llllll}2-2 & 3 & 1.25135 & 0.00908 & 1.2321 & 1.2706\end{array}$

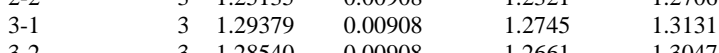

$\begin{array}{llllll}3-1 & 3 & 1.28540 & 0.00908 & 1.2661 & 1.3047 \\ 4-1 & 3 & 1.28960 & 0.00908 & 1.2703 & 1.3089\end{array}$

$\begin{array}{llllll}4-1 & 3 & 1.28960 & 0.00908 & 1.2703 & 1.3089 \\ 4-2 & 3 & 1.28913 & 0.00908 & 1.2699 & 1.3084\end{array}$

Std Error uses a pooled estimate of error variance
Oneway Analysis of $\mathrm{CaO}$ (v) By Analytical Blks (lm)

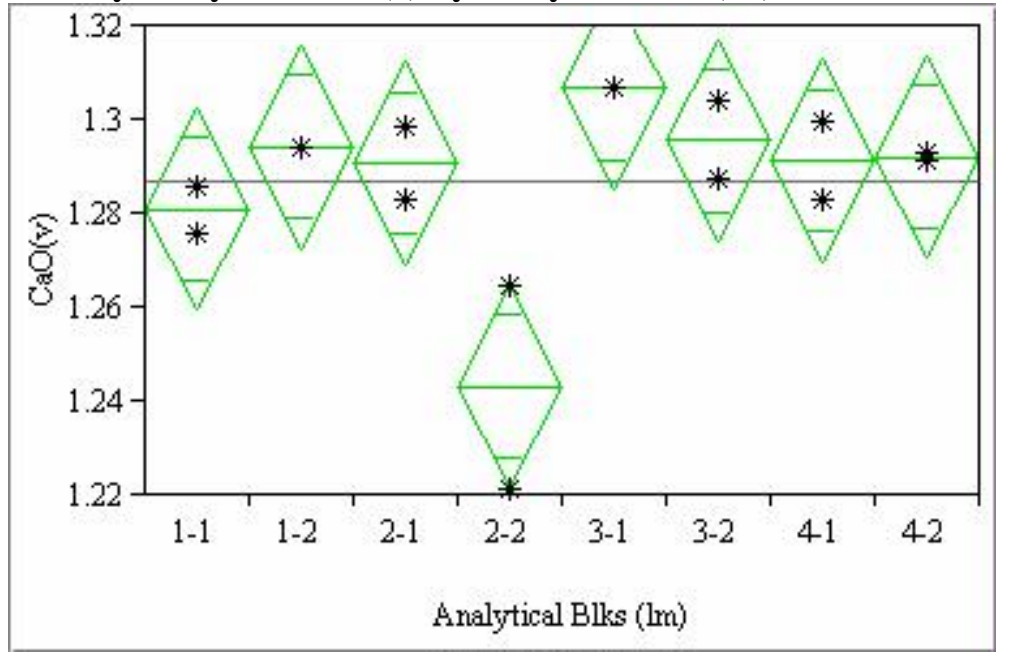

\begin{tabular}{lrrrrr}
\hline $\begin{array}{l}\text { Oneway Anova } \\
\text { Summary of Fit }\end{array}$ & & & & \\
Rsquare & 0.785044 & & & \\
Adj Rsquare & 0.596957 & & & \\
$\begin{array}{l}\text { Root Mean Square Error } \\
\text { Mean of Response }\end{array}$ & 0.013181 & & & & \\
$\begin{array}{l}\text { Observations (or Sum Wgts) } \\
\text { Analysis of Variance }\end{array}$ & 1.286914 & & & & \\
Source & DF & Sum of Squares & Mean Square & F Ratio & Prob > F \\
Analytical Blks (lm) & 7 & 0.00507647 & 0.000725 & 4.1738 & 0.0314 \\
Error & 8 & 0.00139001 & 0.000174 & & \\
C. Total & 15 & 0.00646648 & & &
\end{tabular}

0.00646648

$\begin{array}{lrrrrr}\text { Means for Oneway Anova } & & & \\ \text { Level } & \text { Number } & \text { Mean } & \text { Std Error } & \text { Lower 95\% } & \text { Upper 95\% } \\ 1-1 & 2 & 1.28097 & 0.00932 & 1.2595 & 1.3025\end{array}$

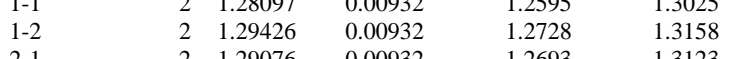

$\begin{array}{llllll}2-1 & 2 & 1.29076 & 0.00932 & 1.2693 & 1.3123 \\ 2-2 & 2 & 1.24319 & 0.00932 & 1.2217 & 1.2647\end{array}$

$\begin{array}{llllll}2-2 & 2 & 1.24319 & 0.00932 & 1.2217 & 1.2647 \\ 3-1 & 2 & 1.30685 & 0.00932 & 1.2854 & 1.3283 \\ 3-2 & 2 & 1.29566 & 0.00932 & 1.2742 & 1.3172\end{array}$

$\begin{array}{llllll}3-2 & 2 & 1.29566 & 0.00932 & 1.2742 & 1.3172 \\ 4-1 & 2 & 1.29146 & 0.00932 & 1.2700 & 1.3130\end{array}$

$\begin{array}{llllll}4-1 & 2 & 1.29146 & 0.00932 & 1.2700 & 1.3130 \\ 4-2 & 2 & 1.29216 & 0.00932 & 1.2707 & 1.3137\end{array}$

Std Error uses a pooled estimate of error variance 
Exhibit E3. Measurements of Standards Prepared Using the Lithium Metaborate (LM) Method by Oxide by Analytical Block

(Small Square - Batch 1 and Asterisk - Ustd)

(Continued)

Oneway Analysis of Cr2O3(v) By Analytical Blks (lm)

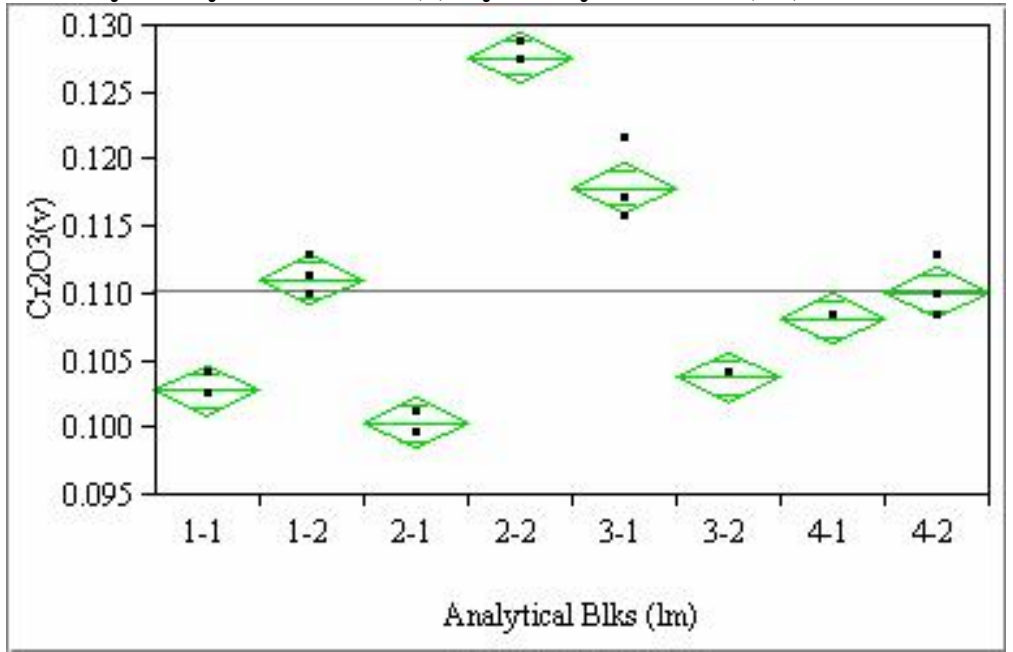

\section{Oneway Anova}

Adj Rsquare

0.97849
0.96908

$\quad 0.001521$

0.110229

Analysis of Variance

$\begin{array}{lllll}\text { Source } & \text { DF Sum of Squares Mean Square F Ratio Prob }>\text { F }\end{array}$

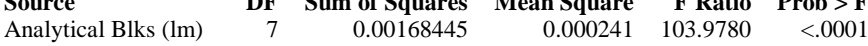

$\begin{array}{lll}\text { Enalytical Blks }(\mathrm{lm}) & 16 & 0.00168445 \\ \text { Error } & 16 & 0.00003703\end{array}$

0.000002

$23-0.00172148$

Means for Oneway Anova

Level Number Mean Std Error $\quad$ Lower 95\% $\quad$ Upper 95\%

$\begin{array}{llllll}1-1 & 3 & 0.102799 & 0.00088 & 0.10094 & 0.10466\end{array}$

$\begin{array}{llllll}1-2 & 3 & 0.111082 & 0.00088 & 0.10922 & 0.11294\end{array}$

$\begin{array}{llllll}2-1 & 3 & 0.100363 & 0.00088 & 0.09850 & 0.10223 \\ 3-1 & 3 & 0.127646 & 0.00088 & 0.12578 & 0.12951\end{array}$

$\begin{array}{llllll}3-1 & 3 & 0.117902 & 0.00088 & 0.12578 & 0.12951 \\ 3-2 & 3 & 0.103774 & 0.00088 & 0.11604 & 0.11976\end{array}$

$\begin{array}{llllll}3-2 & 3 & 0.103774 & 0.00088 & 0.110191 & 0.11976 \\ 4 & 3 & 0.108158 & 0.00088 & 0.10630 & 0.11002\end{array}$

$\begin{array}{llllll}4-1 & 3 & 0.108158 & 0.00088 & 0.10630 & 0.11002 \\ 4-2 & 3 & 0.110107 & 0.00088 & 0.10825 & 0.11197\end{array}$

Std Error uses a pooled estimate of error variance
Oneway Analysis of Cr2O3(v) By Analytical Blks (lm)

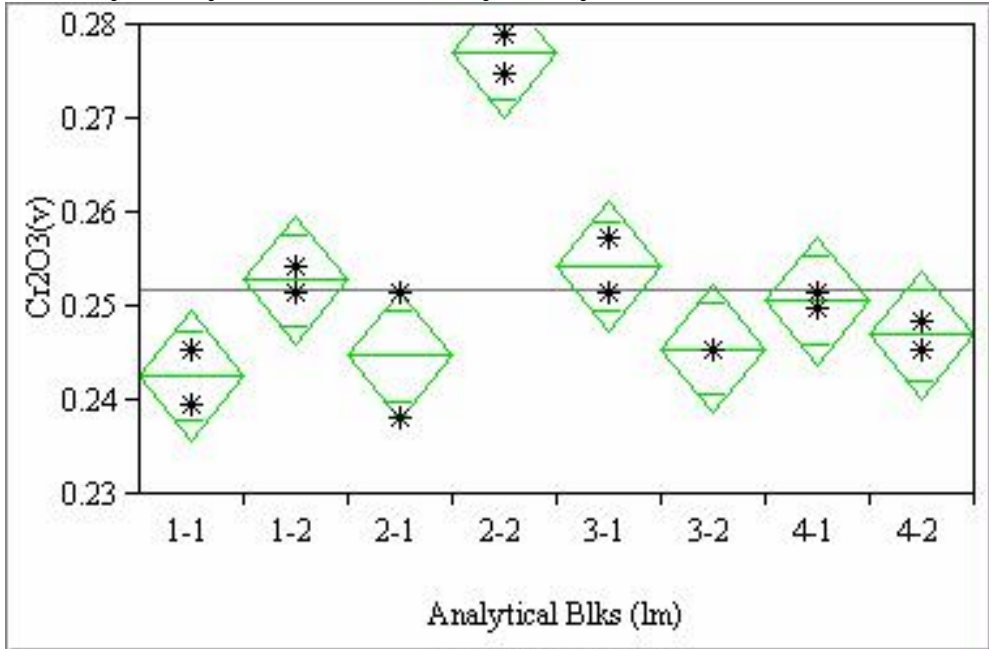

\begin{tabular}{lrrrrr}
\hline $\begin{array}{l}\text { Oneway Anova } \\
\text { Summary of Fit }\end{array}$ & & & & \\
Rsquare & 0.92289 & & & \\
Adj Rsquare & 0.855419 & & & \\
$\begin{array}{l}\text { Root Mean Square Error } \\
\text { Mean of Response }\end{array}$ & 0.004182 & & & \\
$\begin{array}{l}\text { Observations (or Sum Wgts) } \\
\text { Analysis of Variance }\end{array}$ & 0.251852 & & & & \\
Source & DF & Sum of Squares & Mean Square & F Ratio & Prob > F \\
Analytical Blks (lm) & 7 & 0.00167471 & 0.000239 & 13.6783 & 0.0007 \\
Error & 8 & 0.00013993 & 0.000017 & & \\
C. Total & 15 & 0.00181463 & & &
\end{tabular}

Means for Oneway Anova

Level Number Mean Std Error Lower 95\% Upper 95\%

$\begin{array}{llllll}1-1 & 2 & 0.242626 & 0.00296 & 0.23581 & 0.24945 \\ 1-2 & 2 & 0.252857 & 0.00296 & 0.24604 & 0.25968\end{array}$

$\begin{array}{llllll}1-2 & 2 & 0.252857 & 0.00296 & 0.24604 & 0.25968 \\ 2-1 & 2 & 0.244818 & 0.00296 & 0.23800 & 0.25164\end{array}$

$\begin{array}{llllll}2-1 & 2 & 0.244818 & 0.00296 & 0.23800 & 0.25164 \\ 2-2 & 2 & 0.276973 & 0.00296 & 0.27015 & 0.28379\end{array}$

$\begin{array}{llllll}3-1 & 2 & 0.254318 & 0.00296 & 0.24750 & 0.26114 \\ 3-2 & 2 & 0.245549 & 0.00296 & 0.23873 & 0.25237\end{array}$

$\begin{array}{llllll}3-2 & 2 & 0.250664 & 0.00296 & 0.24384 & 0.25748 \\ 4-1 & 2 & 0.24706 & 0.00296 & 0.248 & 0.25383\end{array}$

$0.24019-0.25383$ 
Exhibit E3. Measurements of Standards Prepared Using the Lithium Metaborate (LM) Method by Oxide by Analytical Block

(Small Square - Batch 1 and Asterisk - Ustd)

(Continued)

Oneway Analysis of Fe2O3(v) By Analytical Blks (lm)

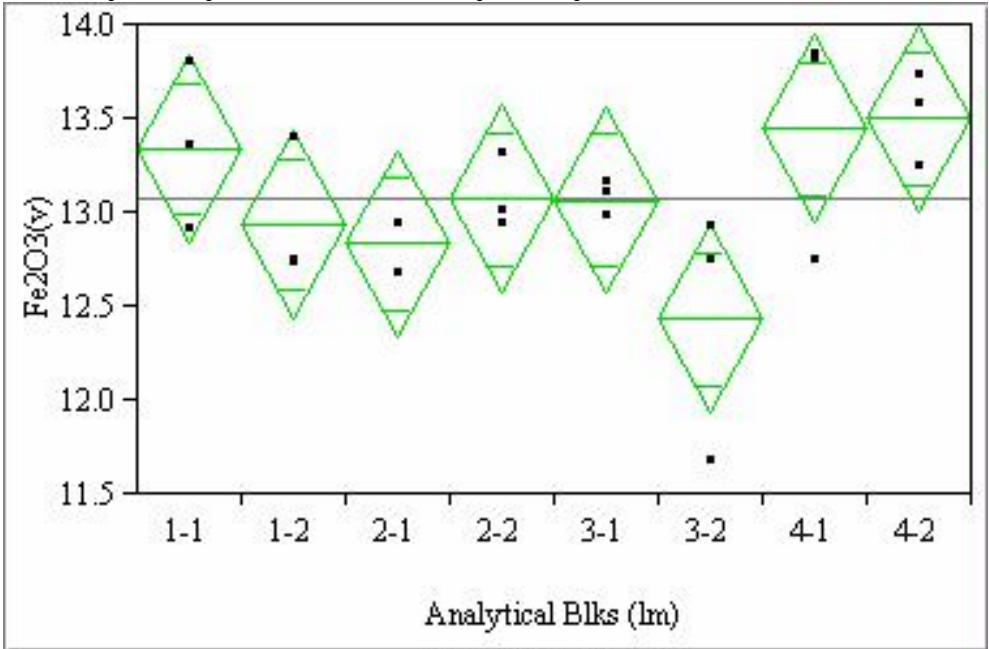

\section{Oneway Anova}

Rsquare

Adj Rsquare

0.499394

Adj Rsquare

0.280378
0.406449
13.07937

Root Mean Square

13.07937

Observations (or Sum Wgts)

Analysis of Variance

Source

Error

C. Total

$\begin{array}{ccccc}7 & \text { Sum of Squares Mean Square F Ratio Prob > F }\end{array}$

2.6368143

0.165201

5.2800333

Level Number Mean Std Error Lower 95\% Upper 95\%

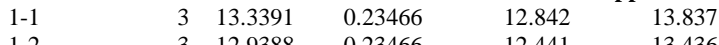

$\begin{array}{llllll}2-1 & 3 & 12.9388 & 0.23466 & 12.441 & 13.436 \\ 2-2 & 3 & 12.8339 & 0.23466 & 12.336 & 13.331\end{array}$

$\begin{array}{llllll}2-2 & 3 & 13.0722 & 0.23466 & 12.575 & 13.570 \\ 3-1 & 3 & 13.0675 & 0.23466 & 12.570 & 13.565\end{array}$

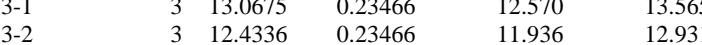

$\begin{array}{llllll}4-1 & 3 & 13.4487 & 0.23466 & 12.951 & 13.946\end{array}$

$\begin{array}{llllll}4-2 & 3 & 13.5011 & 0.23466 & 13.004 & 13.946\end{array}$

Std Error uses a pooled estimate of error variance
Oneway Analysis of Fe2O3(v) By Analytical Blks (lm)

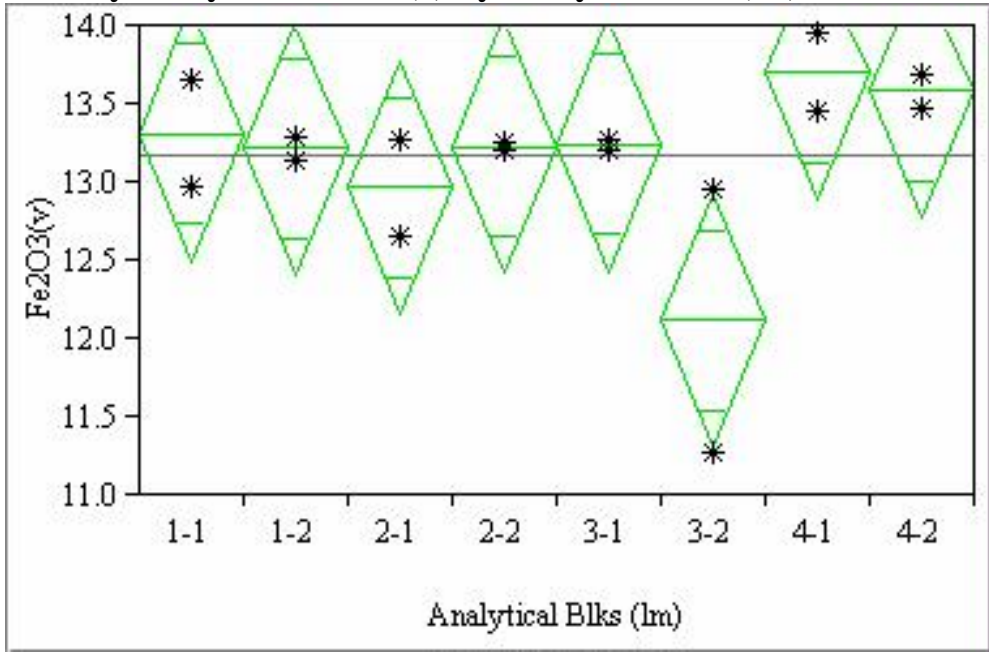

\section{Oneway Anova}

Rsquare

0.622016

Adj Rsquare

0.622016

Root Mean Square Error $\quad 0.499552$

Mean of Response 13.1729

Observations (or Sum Wots)

$\begin{array}{lllll}\begin{array}{l}\text { Analysis of Variance } \\ \text { Source }\end{array} \text { DF Sum of Squares } & \text { Mean Square } & \text { F Ratio Prob > F }\end{array}$

$\begin{array}{lrrrrr}\text { Source } & \text { DF } & \text { Sum of Squares } & \text { Mean Square } & \text { F Ratio } & \text { Prob > F } \\ \text { Analytical Blks (lm) } & 7 & 3.2853378 & 0.469334 & 1.8807 & 0.1976\end{array}$

$\begin{array}{lrrr}\text { Error } & 8 & 1.9964159 & 0.249552\end{array}$

$15-5.2817537$

Level Number Mean Std Error Lower 95\% Upper 95\%

$\begin{array}{lrrrr}\text { Level Number } & \text { Mean } & \text { Std Error } & \text { Lower 95\% } & \text { Upper 95\% } \\ 1-1 & 2 & 13.3105 & 0.35324 & 12.496\end{array}$

$\begin{array}{llllll}1-1 & 2 & 13.3105 & 0.35324 & 12.496 & 14.125 \\ 1-2 & 2 & 13.2176 & 0.35324 & 12.403 & 14.032 \\ 2-1 & 2 & 12.9674 & 0.35324 & 12.153 & 13.782\end{array}$

$\begin{array}{llllll}2-1 & 2 & 12.9674 & 0.35324 & 12.153 & 13.782 \\ 2-2 & 2 & 13.2319 & 0.35324 & 12.417 & 14.046\end{array}$

$\begin{array}{llllll}2-2 & 2 & 13.2319 & 0.35324 & 12.417 & 14.046 \\ 3-1 & 2 & 13.2462 & 0.35324 & 12.432 & 14.061 \\ 3-2 & 2 & 12.1167 & 0.35324 & 11.302 & 12.931\end{array}$

$\begin{array}{llllll}3-2 & 2 & 12.1167 & 0.35324 & 11.302 & 12.931 \\ 4-1 & 2 & 13.7037 & 0.35324 & 12.889 & 14.518 \\ 4-2 & 2 & 13.5893 & 0.35324 & 12.775 & 14.404\end{array}$

Std Error uses a pooled estimate of error variance 
Exhibit E3. Measurements of Standards Prepared Using the Lithium Metaborate (LM) Method by Oxide by Analytical Block

(Small Square - Batch 1 and Asterisk - Ustd)

(Continued)

Oneway Analysis of MgO(v) By Analytical Blks (lm)

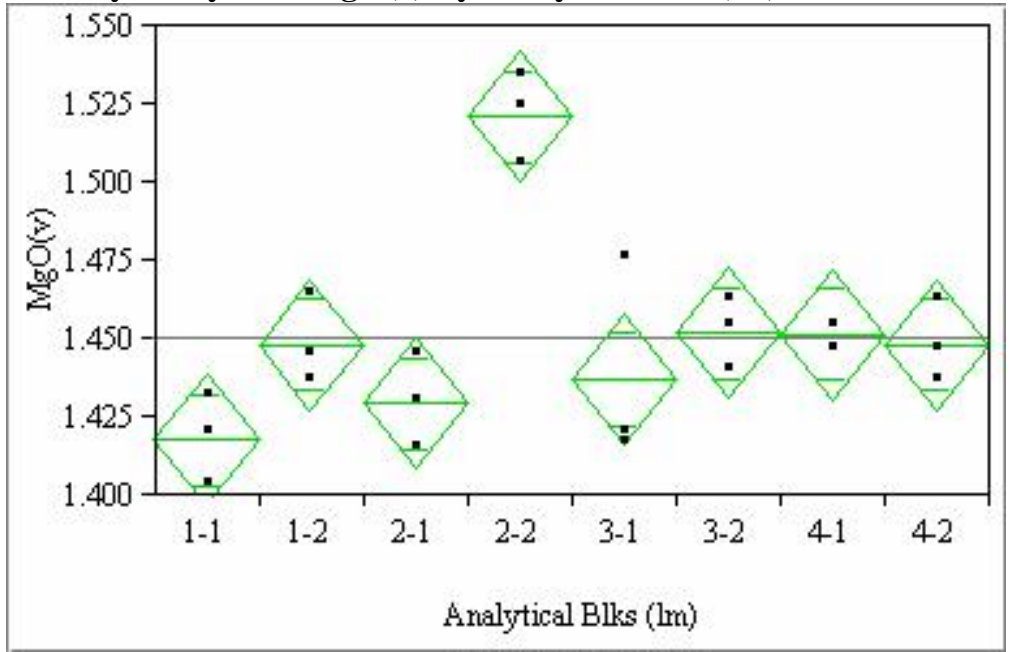

Oneway Anova

(usquare

Adj Rsquare

0.813474

Adj Rsquare

0.731869

1.450561

Mean of Response

Observations (or Sum Wgts)

Source

$\begin{array}{lrrrrr}\text { Source } & \text { DF } & \text { Sum of Squares } & \text { Mean Square } & \text { F Ratio } & \text { Prob > F } \\ \text { Analytical Blks (lm) } & 7 & 0.02009552 & 0.002871 & 9.9684 & <.0001\end{array}$

Error

C. Total

Sum of Squares Mean Square F Ratio Prob >

$16-0.00460782-0.000288$

0.02470334

Level Number Mean Std Error Lower 95\% Upper 95\%

$\begin{array}{llllll}1-1 & 3 & 1.41768 & 0.00980 & 1.3969 & 1.4384\end{array}$

$\begin{array}{llllll}1-2 & 3 & 1.44807 & 0.00980 & 1.4273 & 1.4688 \\ 2-1 & 3 & 1.42928 & 0.00980 & 1.4085 & 1.4501\end{array}$

$\begin{array}{llllll}2-2 & 3 & 1.52103 & 0.0080 & 1.4085 & 1.4501 \\ 3-1 & 3 & 1.43702 & 0.0080 & 1.5003 & 1.5418\end{array}$

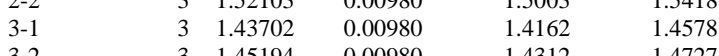

$\begin{array}{llllll}3-1 & 3 & 1.45194 & 0.00980 & 1.4312 & 1.4727 \\ 4-1 & 3 & 1.45139 & 0.00980 & 1.4306 & 1.4722\end{array}$

$\begin{array}{llllll}4-1 & 3 & 1.45139 & 0.00980 & 1.4306 & 1.4722 \\ 4-2 & 3 & 1.44807 & 0.00980 & 1.4273 & 1.4688\end{array}$

Std Error uses a pooled estimate of error variance
Oneway Analysis of MgO(v) By Analytical Blks (Im)

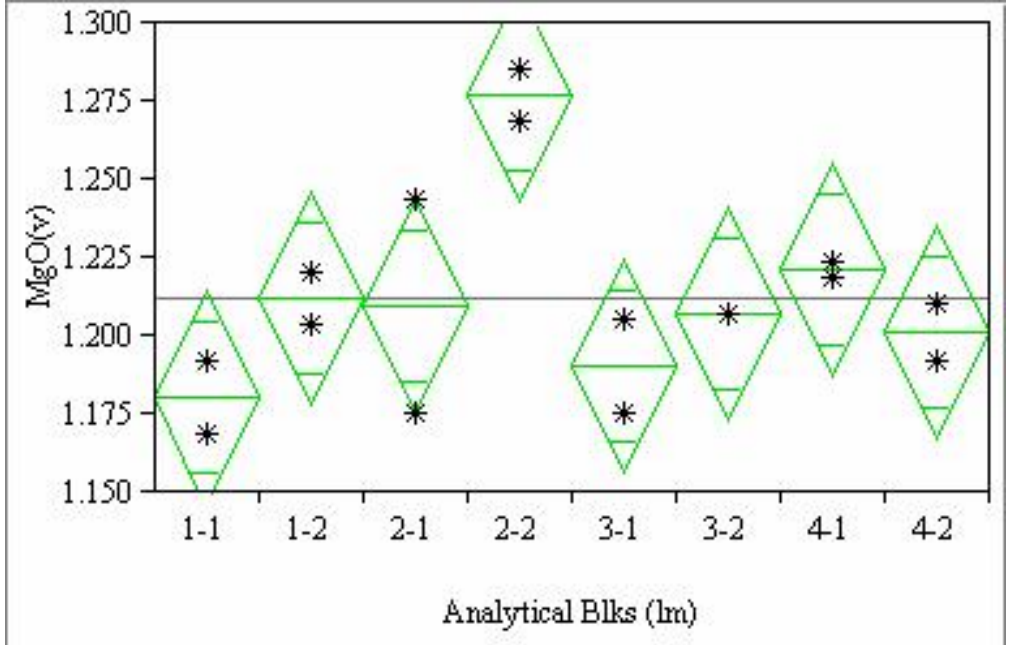

Oneway Anova

$\begin{array}{ll}\text { Summary of Fit } & 0.771341 \\ \text { Rsquare } & 0.571264\end{array}$

$\begin{array}{ll}\text { Adj Rsquare } & 0.571264 \\ \text { Root Mean Square Error } & 0.020854\end{array}$

Root Mean Square Error $\quad 0.020854$

\begin{tabular}{lr} 
Observations (or Sum Wots) & 1.212382 \\
\hline
\end{tabular}

Analysis of Variance

$\begin{array}{lllll}\text { Source } & \text { DF Sum of Squares Mean Square } & \text { F Ratio Prob }>\text { F }\end{array}$

$\begin{array}{lrrrrr}\text { Analytical Blks (lm) } & 7 & 0.01173657 & 0.001677 & 3.8552 & 0.0389\end{array}$

$\begin{array}{lrrr}\text { Error } & 8 & 0.00347923 & 0.000435\end{array}$

C. Total $15 \quad 0.01521580$

Means for Oneway Anova

Level Number Mean Std Error Lower 95\% Upper 95\%

$\begin{array}{lrrrrr}\text { Level } & \text { Number } & \text { Mean } & \text { Std Error } & \text { Lower 95\% } & \text { Upper 95\% } \\ 1-1 & 2 & 1.18057 & 0.01475 & 1.1466 & 1.2146\end{array}$

$\begin{array}{llllll}1-1 & 2 & 1.18057 & 0.01475 & 1.1466 & 1.2146 \\ 1-2 & 2 & 1.21207 & 0.01475 & 1.1781 & 1.2461 \\ 2-1 & 2 & 1.20958 & 0.01475 & 1.1756 & 1.2436\end{array}$

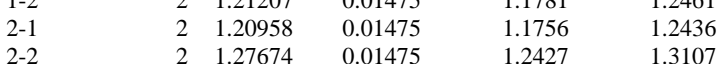

$\begin{array}{llllll}2-2 & 2 & 1.27674 & 0.01475 & 1.2427 & 1.3107 \\ 3-1 & 2 & 1.19052 & 0.01475 & 1.1565 & 1.2245 \\ 3-2 & 2 & 1.20710 & 0.01475 & 1.1731 & 1.2411\end{array}$

$\begin{array}{llllll}3-2 & 2 & 1.20710 & 0.01475 & 1.1731 & 1.2411 \\ 4-1 & 2 & 1.22119 & 0.01475 & 1.1872 & 1.2552\end{array}$

$\begin{array}{llllll}4-1 & 2 & 1.22119 & 0.01475 & 1.1872 & 1.2552 \\ 4-2 & 2 & 1.20129 & 0.01475 & 1.1673 & 1.2353\end{array}$

Std Error uses a pooled estimate of error variance 
Exhibit E3. Measurements of Standards Prepared Using the Lithium Metaborate (LM) Method by Oxide by Analytical Block

(Small Square - Batch 1 and Asterisk - Ustd)

(Continued)

Oneway Analysis of MnO(v) By Analytical Blks (lm)

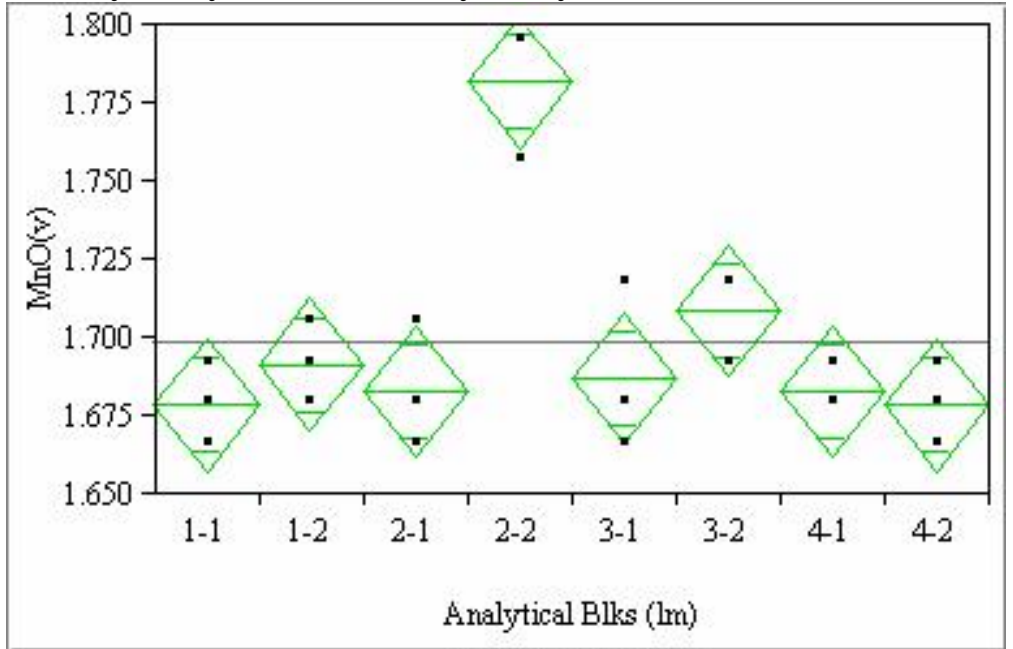

\section{Oneway Anova}

Sumare

Adj Rsquare

0.842346

0.773373
0.017283

Root Mean Square Error $\quad 0.017283$

Observations (or Sum Wgts)

Analysis of Variance

Source DF Sum of Squares Mean Square F Ratio Prob > F

$\begin{array}{lrrrrr}\text { Analytical Blks (lm) } & 7 & 0.02553591 & 0.003648 & 12.2126 & <.0001 \\ & 16 & 0.00477930 & 0.000299 & & \end{array}$

Error

0.003648

Means for Oneway Anova

0.03031521

or Oneway Anova

Level Number Mean Std Error Lower 95\% Upper 95\%

$\begin{array}{llllll}1-1 & 3 & 1.67856 & 0.00998 & 1.6574 & 1.6997 \\ 1-2 & 3 & 1.69147 & 0.00998 & 1.6703 & 1.7126\end{array}$

$\begin{array}{llllll}2-1 & 3 & 1.68286 & 0.00998 & 1.6617 & 1.7040\end{array}$

$\begin{array}{llllll}2-2 & 3 & 1.78186 & 0.00998 & 1.7607 & 1.8030 \\ 3-1 & 3 & 1.68717 & 0.00998 & 1.6660 & 1.7089\end{array}$

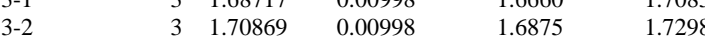

$\begin{array}{llllll}4-1 & 3 & 1.68286 & 0.00998 & 1.6617 & 1.7040\end{array}$

$\begin{array}{lll}3 & 1.67856 & 0.00998\end{array}$

$1.6574-1.6997$

Std Error uses a pooled estimate of error variance
Oneway Analysis of MnO(v) By Analytical Blks (lm)

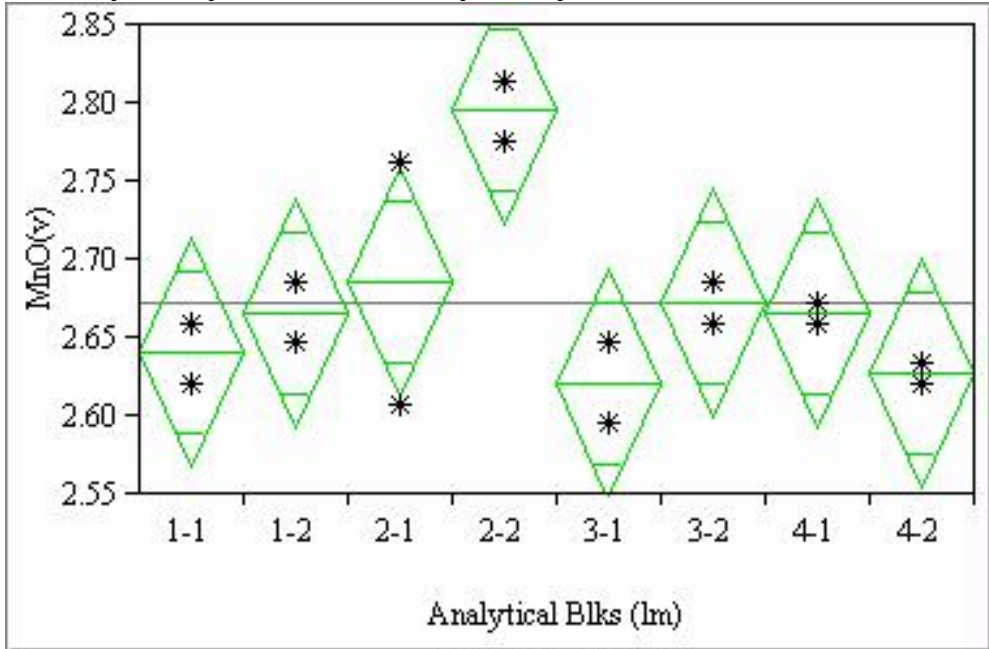

Oneway Anova

Summary of

Rsquare

0.723446

Adj Rsquare $\quad 0.481462$

Root Mean Square Error $\quad 0.044845$

Mean of Response

2.671977

Observations (or Sum Wgts)

$\begin{array}{lllll}\text { Source } & \text { DF Sum of Squares Mean Square } & \text { F Ratio Prob }>\text { F }\end{array}$

$\begin{array}{lrrrrr}\text { Analytical Blks }(\mathrm{lm}) & 7 & 0.04208632 & 0.006012 & 2.9896 & 0.0739\end{array}$

$\begin{array}{llll}\text { Error } & 8 & 0.01608846 & 0.002011\end{array}$

$15 \quad 0.05817477$

Means for Oneway Anova

Level Number Mean Std Error Lower 95\% Upper 95\%

$\begin{array}{lrrrrr}1-1 & 2 & 2.64050 & 0.03171 & 2.5674 & 2.7136 \\ 1-2 & 2 & 2.6633 & 0.03171 & 2.5932 & 2.7395\end{array}$

$\begin{array}{llllll}1-2 & 2 & 2.66633 & 0.03171 & 2.5932 & 2.7395 \\ 2-1 & 2 & 2.68570 & 0.03171 & 2.6126 & 2.7588\end{array}$

$\begin{array}{llllll}2-1 & 2 & 2.68570 & 0.03171 & 2.6126 & 2.7588 \\ 2-2 & 2 & 2.79545 & 0.03171 & 2.7223 & 2.8686 \\ 3-1 & 2 & 2.62114 & 0.03171 & 2.5480 & 2.6943\end{array}$

$\begin{array}{llllll}3-1 & 2 & 2.62114 & 0.03171 & 2.5480 & 2.6943 \\ 3-2 & 2 & 2.67278 & 0.03171 & 2.5997 & 2.7459\end{array}$

$\begin{array}{llllll}3-2 & 2 & 2.66633 & 0.03171 & 2.5997 & 2.7459 \\ 4-1 & 2 & 2.62759 & 0.03171 & 2.552 & 2.7395\end{array}$

$\begin{array}{ll}2.5545 & 2.7007\end{array}$ 
Exhibit E3. Measurements of Standards Prepared Using the Lithium Metaborate (LM) Method by Oxide by Analytical Block

Oneway Analysis of $\mathrm{Na2O}(\mathrm{v})$ By Analytical Blks (lm)

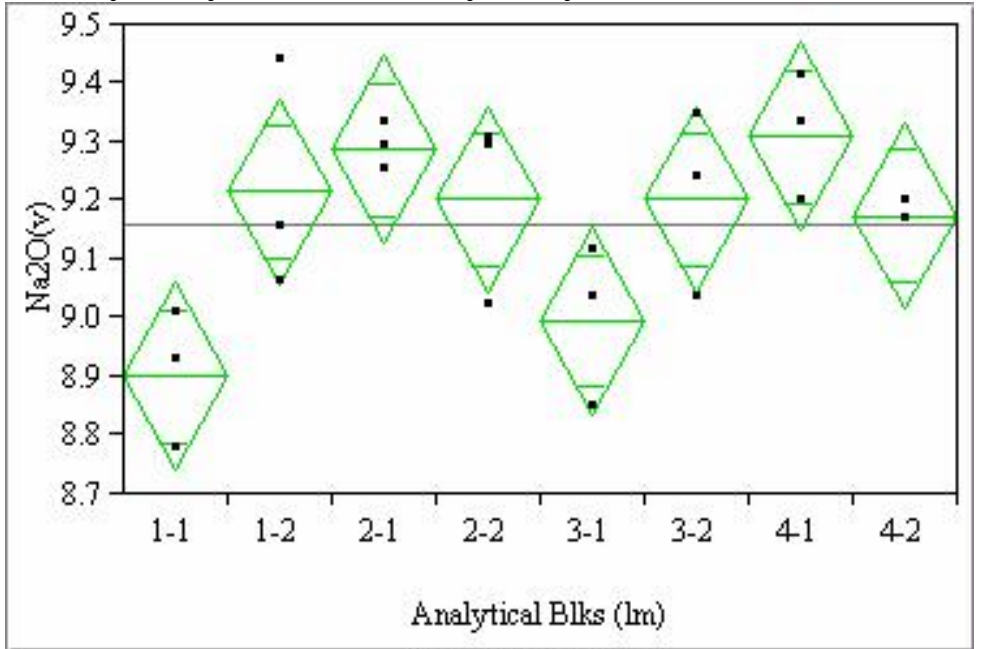

\section{Oneway Anova}

Rsquare

Adj Rsquare

Root Mean Square

0.436204

Root Mean Square

9.161345

Observations (or Sum Wgts)

Analysis of Variance

Source DF Sum of Squares Mean Square F Ratio Prob > F

$\begin{array}{lrllll}\text { Analytical Blks (lm) } & 7 & 0.41919832 & 0.059885 & 3.5421 & 0.0171 \\ & 16 & 0.27050622 & 0.016907 & & \end{array}$

0.016907

Ceans for Oneway Anova

Level Number Mean Std Error Lower 95\% Upper 95\%

$\begin{array}{lrrrrr}\text { Level } & \text { Number } & \text { Mean } & \text { Std Eror } & \text { Lower 95\% } & \text { Upper 95\% } \\ 1-1 & 3 & 8.90129 & 0.07507 & 8.7422 & 9.0604\end{array}$

$\begin{array}{llllll}1-1 & 3 & 8.9129 & 0.07507 & 8.7422 & 9.0604 \\ 1-2 & 3 & 9.21583 & 0.07507 & 9.0567 & 9.3750\end{array}$

$\begin{array}{llllll}1-1 & 3 & 9.28772 & 0.07507 & 9.1286 & 9.4469 \\ 2-2 & 3 & 9.20235 & 0.07507 & 9.0432 & 9.3615\end{array}$

$\begin{array}{llllll}2-2 & 3 & 9.20235 & 0.07507 & 9.0432 & 9.3615 \\ 3-1 & 3 & 8.99565 & 0.07507 & 8.8365 & 9.1548\end{array}$

$\begin{array}{llllll}3-2 & 3 & 9.20235 & 0.07507 & 9.0432 & 9.15615 \\ 4 & 3 & 9.3100 & 0.07507 & 9.1510 & 9.463\end{array}$

$\begin{array}{llllll}4-1 & 3 & 9.31019 & 0.07507 & 9.1510 & 9.4693\end{array}$

$\begin{array}{lll}3 & 9.17539 & 0.07507\end{array}$

$9.0162-9.3345$

Std Error uses a pooled estimate of error variance
Oneway Analysis of $\mathrm{Na2O}(\mathrm{v})$ By Analytical Blks (Im)

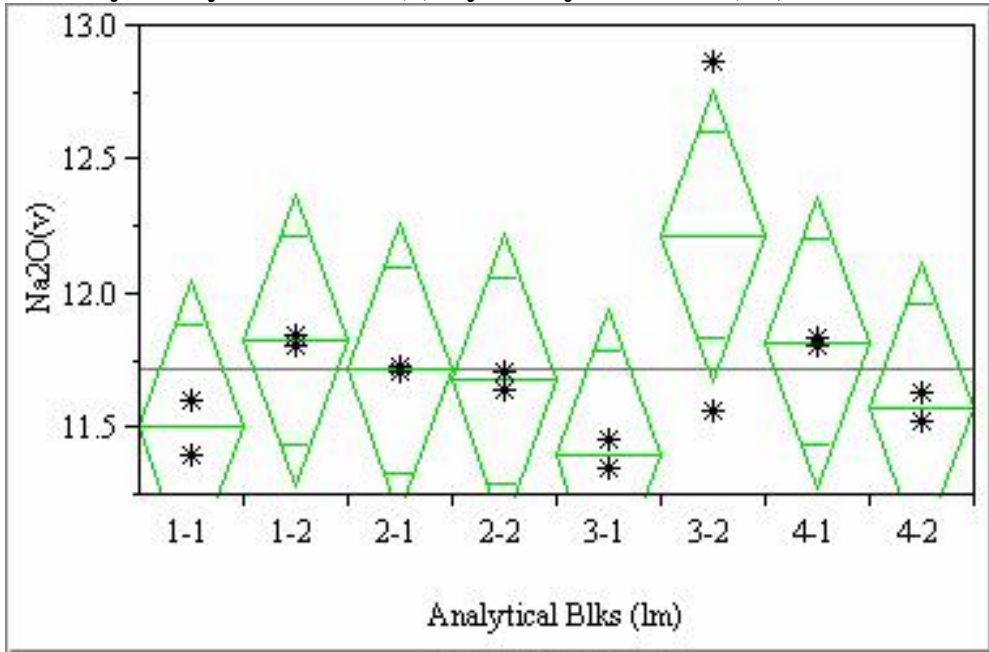

\section{Oneway Anova}

Rsquare

0.496555

Adj Rsquare

0.496555

$\begin{array}{lr}\text { Adj Rsquare } & 0.056041 \\ \text { Root Mean Square Error } \quad 0.33363\end{array}$

$\begin{array}{lr}\text { Root Mean Square Error } & 0.33363 \\ \text { Mean of Response } & 11.72002\end{array}$

Observations (or Sum Wgts)

Analysis of Variance

$\begin{array}{lllll}\text { Source } & \text { DF Sum of Squares Mean Square } & \text { F Ratio } & \text { Prob }>\text { F }\end{array}$

$\begin{array}{lrrrrr}\text { Source } & \text { DF } & \text { Sum of Squares } & \text { Mean Square } & \text { F Ratio } & \text { Prob > F } \\ \text { Analytical Blks (lm) } & 7 & 0.8782859 & 0.125469 & 1.1272 & 0.4306\end{array}$

$\begin{array}{lrrr}\text { Error } & 8 & 0.8904718 & 0.111309 \\ \text { C. Total } & 15 & 1.7687577 & \end{array}$

Level Number $\quad$ Std Error Lower 95\% Upper 95\%

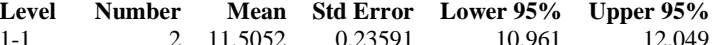

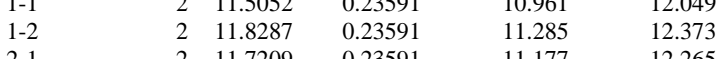

$\begin{array}{llllll}2-1 & 2 & 11.7209 & 0.23591 & 11.177 & 12.265 \\ 2-2 & 2 & 11.6804 & 0.23591 & 11.136 & 12.224\end{array}$

$\begin{array}{llllll}2-2 & 2 & 11.6804 & 0.23591 & 11.136 & 12.224 \\ 3-1 & 2 & 11.4041 & 0.23591 & 10.860 & 11.948 \\ 3-2 & 2 & 12.2196 & 0.23591 & 11.676 & 12.764\end{array}$

$\begin{array}{llllll}3-2 & 2 & 12.2196 & 0.23591 & 11.676 & 12.764 \\ 4-1 & 2 & 11.8220 & 0.23591 & 11.278 & 12.366 \\ 4-2 & 2 & 11.5793 & 0.23591 & 11.035 & 12.123\end{array}$

Std Error uses a pooled estimate of error variance 
Exhibit E3. Measurements of Standards Prepared Using the Lithium Metaborate (LM) Method by Oxide by Analytical Block

(Small Square - Batch 1 and Asterisk - Ustd)

(Continued)

Oneway Analysis of NiO(v) By Analytical Blks (lm)

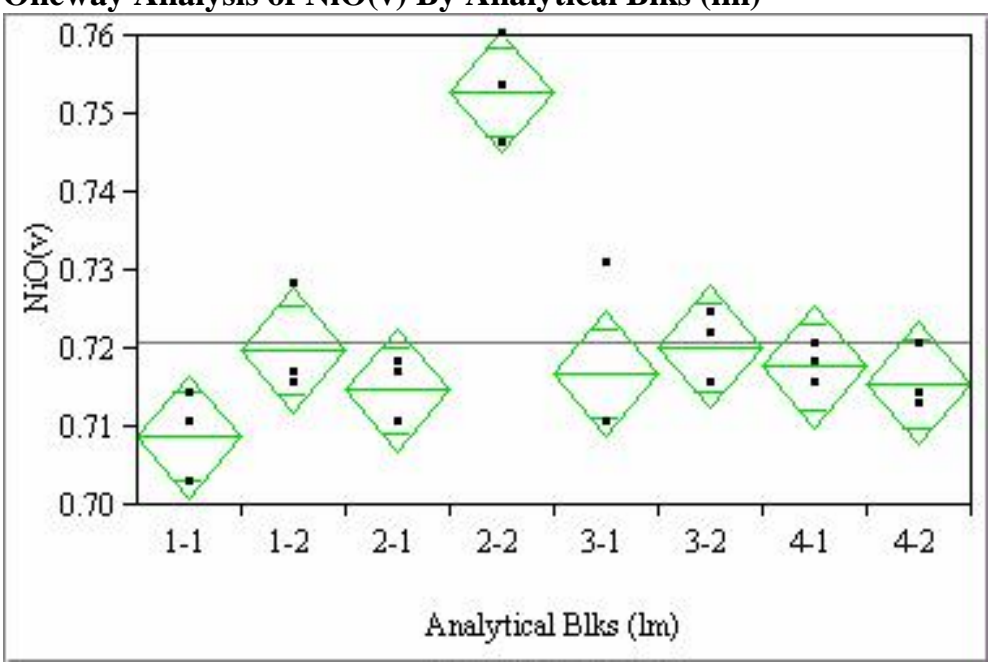

\section{Oneway Anova}

Summary of

Rsquare
Adj Rsquare

0.851365

$\begin{array}{ll}\text { Adj Rsquare } & 0.786338 \\ \text { Root Mean Square Error } & 0.006436\end{array}$

Mean of Response

0.720818
24

Observations (or Sum Wgts)

$\begin{array}{lrrrrr}\text { Source } & \text { DF } & \text { Sum of Squares } & \text { Mean Square } & \text { F Ratio } & \text { Prob > F } \\ \text { Analytical Blks (lm) } & 7 & 0.00379655 & 0.000542 & 13.0924 & <.0001 \\ \text { Error } & 16 & 0.00066282 & 0.000041 & & \end{array}$

0.00445936

C. Total

va

$\begin{array}{lrrrrr}\text { Level } & \text { Number } & \text { Mean } & \text { Std Error } & \text { Lower 95\% } & \text { Upper 95\% } \\ 1-1 & 3 & 0.708782 & 0.00372 & 0.70090 & 0.71666 \\ 1-2 & 3 & 0.719811 & 0.00372 & 0.71193 & 0.72769 \\ 2-1 & 3 & 0.714721 & 0.00372 & 0.70684 & 0.72260 \\ 2-2 & 3 & 0.752896 & 0.00372 & 0.74502 & 0.76077 \\ 3-1 & 3 & 0.716842 & 0.00372 & 0.70896 & 0.72472 \\ 3-2 & 3 & 0.720235 & 0.00372 & 0.71236 & 0.72811 \\ 4-1 & 3 & 0.717690 & 0.00372 & 0.70981 & 0.72557 \\ 4-2 & 3 & 0.715569 & 0.00372 & 0.70769 & 0.72345\end{array}$

Oneway Analysis of $\mathrm{NiO}(\mathrm{v})$ By Analytical Blks (lm)

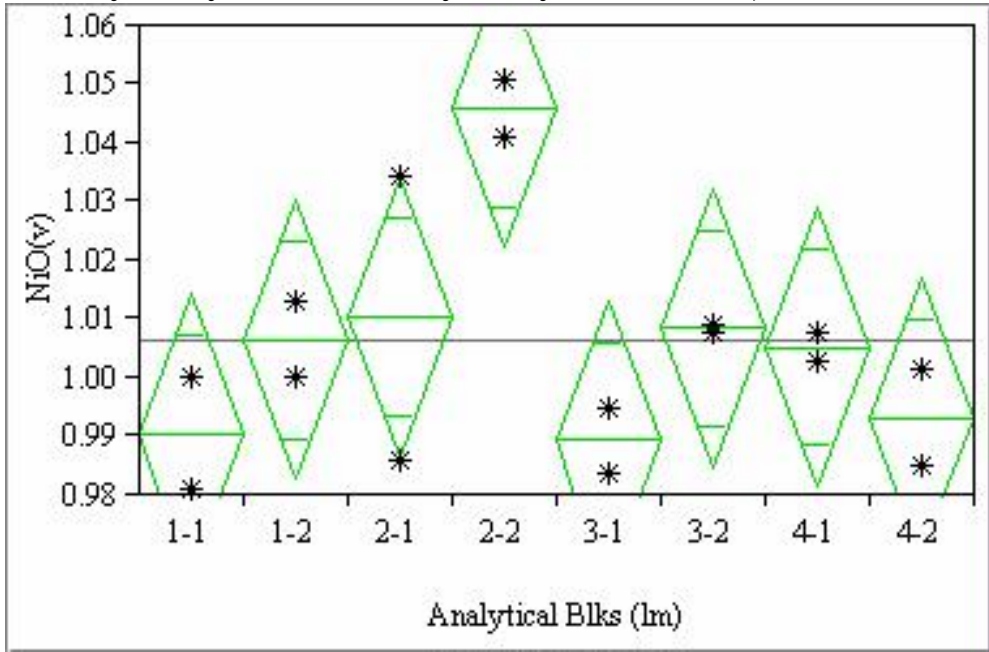

\begin{tabular}{|c|c|c|c|c|c|}
\hline \multicolumn{6}{|l|}{$\begin{array}{l}\text { Oneway Anova } \\
\text { Summary of Fit }\end{array}$} \\
\hline \multicolumn{6}{|l|}{$\begin{array}{l}\text { Rsquare } \\
\text { Rs o Th }\end{array}$} \\
\hline Adj Rsquare & & 0.494285 & & & \\
\hline \multicolumn{2}{|c|}{ Root Mean Square Error } & 0.014578 & & & \\
\hline \multicolumn{2}{|c|}{ Mean of Response } & 1.006229 & & & \\
\hline \multicolumn{2}{|c|}{ Observations (or Sum Wgts) } & 16 & & & \\
\hline \multicolumn{6}{|c|}{ Analysis of Variance } \\
\hline \multicolumn{2}{|c|}{ Source } & Sum of Squares & Mean Square & F Ratio & Prob $>\mathbf{F}$ \\
\hline Analytical Blks (lm) & 7 & 0.00460355 & \multirow{2}{*}{0.000658} & 3.0944 & 0.0680 \\
\hline Error & 8 & 0.00170022 & & & \\
\hline \multirow{2}{*}{\multicolumn{2}{|c|}{$\begin{array}{lr}\text { C. Total } & 15 \\
\text { Means for Oneway Anova }\end{array}$}} & 0.00630376 & 0.000213 & & \\
\hline & & & \multirow[b]{2}{*}{ Lower $95 \%$} & & \\
\hline \multicolumn{2}{|c|}{ Level Number Mean } & Std Error & & er $95 \%$ & \\
\hline $1-1 \quad 2$ & 0.99064 & 0.01031 & 0.9669 & 1.0144 & \\
\hline $1-2$ & 1.00655 & 0.01031 & 0.9828 & 1.0303 & \\
\hline $2-1$ & 1.01037 & 0.01031 & 0.9866 & 1.0341 & \\
\hline $2-2$ & 1.04600 & 0.01031 & 1.0222 & 1.0698 & \\
\hline 3-1 & 0.98937 & 0.01031 & 0.9656 & 1.0131 & \\
\hline $3-2$ & 1.00846 & 0.01031 & 0.9847 & 1.0322 & \\
\hline 4-1 & 1.00528 & 0.01031 & 0.9815 & 1.0290 & \\
\hline $4-2$ & 0.99319 & 0.01031 & 0.9694 & 1.0170 & \\
\hline
\end{tabular}


Exhibit E3. Measurements of Standards Prepared Using the Lithium Metaborate (LM) Method by Oxide by Analytical Block

(Small Square - Batch 1 and Asterisk - Ustd)

(Continued)

Oneway Analysis of SiO2(v) By Analytical Blks (lm)

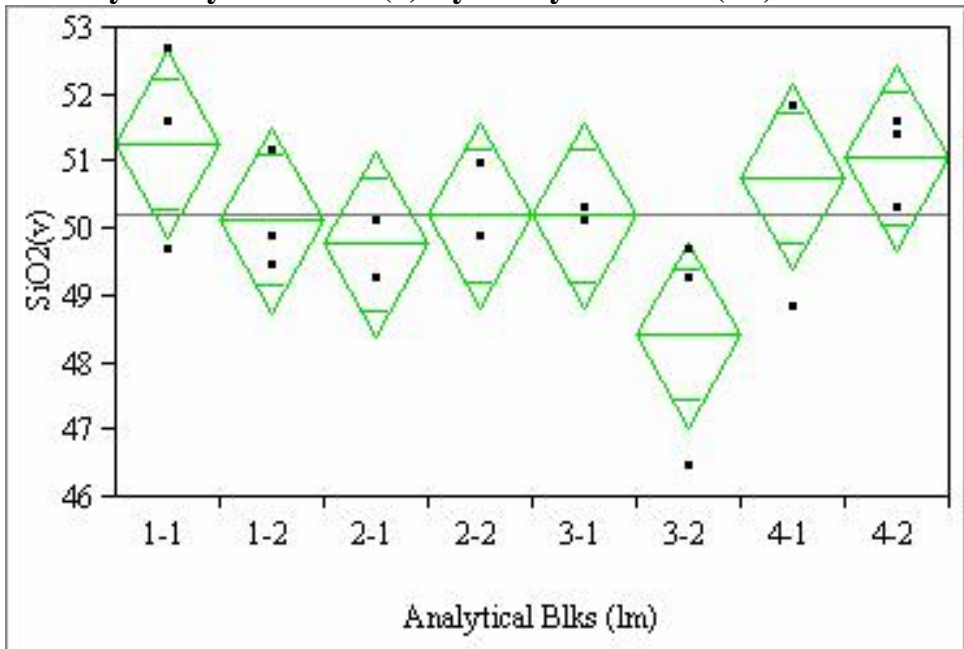

\section{Oneway Anova}

Summary

Adj Rsquare

Root Mean Square

\subsection{9}

0.208036

1.131169

Mean of Response

50.22898
24

Observations (or Sum Wgts)

Analysis of Variance

Source

$\begin{array}{rrrrr}\text { DF } & \text { Sum of Squares } & \text { Mean Square } & \text { F Ratio } & \text { Prob }>\text { F } \\ 7 & 16.687444 & 2.38392 & 1.8631 & 0.1433\end{array}$

Error

$\begin{array}{lll}\text { Error } & 16 & 20.472677 \\ \text { C. Total } & 23 & 37.160122\end{array}$

37.160122

Level Number Mean Std Error Lower 95\% Upper 95\%

$\begin{array}{llllll}1-1 & 3 & 51.2719 & 0.65308 & 49.887 & 52.656 \\ 1-2 & 3 & 50.1309 & 0.65308 & 48.746 & 51.5159\end{array}$

$\begin{array}{llllll}2-1 & 3 & 50.1309 & 0.65308 & 48.746 & 51.515 \\ 2 & 3 & 49.7744 & 0.65308 & 48.390 & 51.159\end{array}$

$\begin{array}{llllll}2-2 & 3 & 50.2022 & 0.65308 & 48.818 & 51.587 \\ 3 & 3 & 50.2022 & 0.6508 & 48.818 & 51.587\end{array}$

$\begin{array}{llllll}3-1 & 3 & 50.2022 & 0.65308 & 48.818 & 51.587 \\ 3-2 & 3 & 48.4195 & 0.65308 & 47.035 & 49.804\end{array}$

$\begin{array}{llllll}3-1 & 3 & 48.4195 & 0.65308 & 47.035 & 49.804 \\ 4-1 & 3 & 50.7727 & 0.65308 & 49.388 & 52.157\end{array}$

$\begin{array}{llllll}4-2 & 3 & 50.7727 & 0.65308 & 49.388 & 52.157 \\ 4-2 & 3 & 51.0580 & 0.65308 & 49.673 & 52.442\end{array}$

Std Error uses a pooled estimate of error variance
Oneway Analysis of SiO2(v) By Analytical Blks (lm)

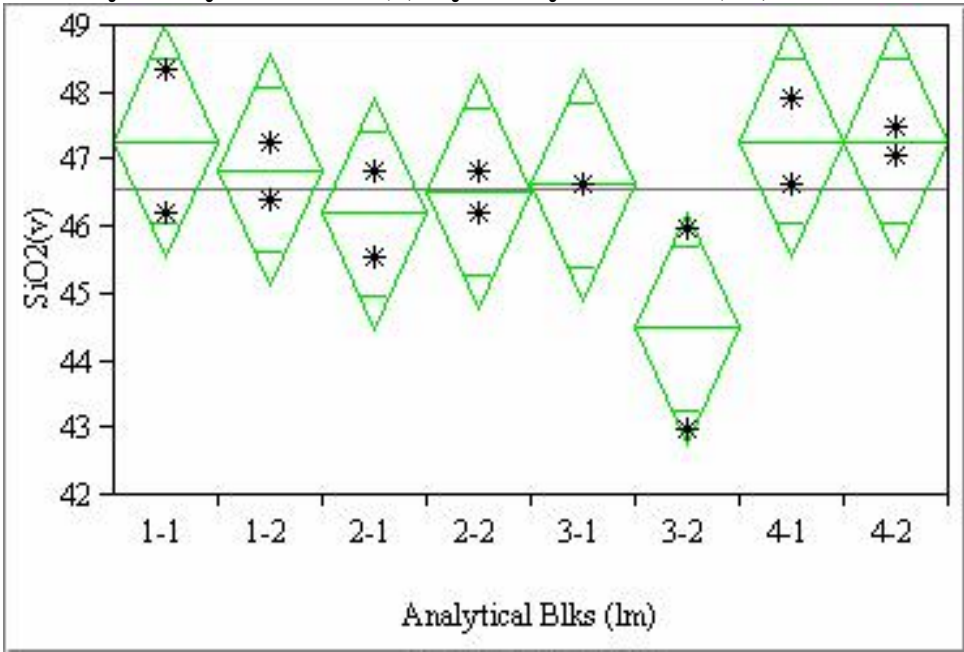

\begin{tabular}{|c|c|c|c|c|c|}
\hline \\
\hline $\begin{array}{l}\text { Summary of Fit } \\
\text { Rsquare }\end{array}$ & & 0.569823 & & & \\
\hline \multicolumn{2}{|l|}{ Adj Rsquare } & 0.193417 & & & \\
\hline \multicolumn{2}{|c|}{ Root Mean Square Error } & 1.065631 & & & \\
\hline \multicolumn{2}{|c|}{ Mean of Response } & 46.56989 & & & \\
\hline \multirow{2}{*}{\multicolumn{6}{|c|}{$\begin{array}{l}\text { Observations (or Sum Wgts) } \\
\text { Analysis of Variance }\end{array}$}} \\
\hline & & & & \\
\hline Source & DF & Sum of Squar & Mean S & guare & F Rat \\
\hline Analytical Blks (lm & 7 & 12.0336 & & 1909 & 1.513 \\
\hline Error & 8 & 9.0845 & & 3557 & \\
\hline C. Total & 15 & $21.1181 \mathrm{C}$ & & & \\
\hline \multicolumn{6}{|c|}{ Means for Oneway Anova } \\
\hline Level Number & Mean & Std Error & Lower 95\% & Upp & er $95 \%$ \\
\hline $1-1$ & 47.2785 & 0.75352 & 45.541 & & 49.016 \\
\hline $1-2$ & 46.8507 & 0.75352 & 45.113 & & 48.588 \\
\hline $2-1$ & 46.2089 & 0.75352 & 44.471 & & 47.946 \\
\hline $2-2$ & 46.5298 & 0.75352 & 44.792 & & 48.267 \\
\hline 3-1 & 46.6367 & 0.75352 & 44.899 & & 48.374 \\
\hline 3-2 & 44.4974 & 0.75352 & 42.760 & & 46.235 \\
\hline $4-1$ & 47.2785 & 0.75352 & 45.541 & & 49.016 \\
\hline 4-2 & 47.2785 & 0.75352 & 45.541 & & 49.016 \\
\hline
\end{tabular}

Std Error uses a pooled estimate of error variance 
Exhibit E3. Measurements of Standards Prepared Using the Lithium Metaborate (LM) Method by Oxide by Analytical Block

(Small Square - Batch 1 and Asterisk - Ustd)

(Continued

Oneway Analysis of ZrO2(v) By Analytical Blks (lm)

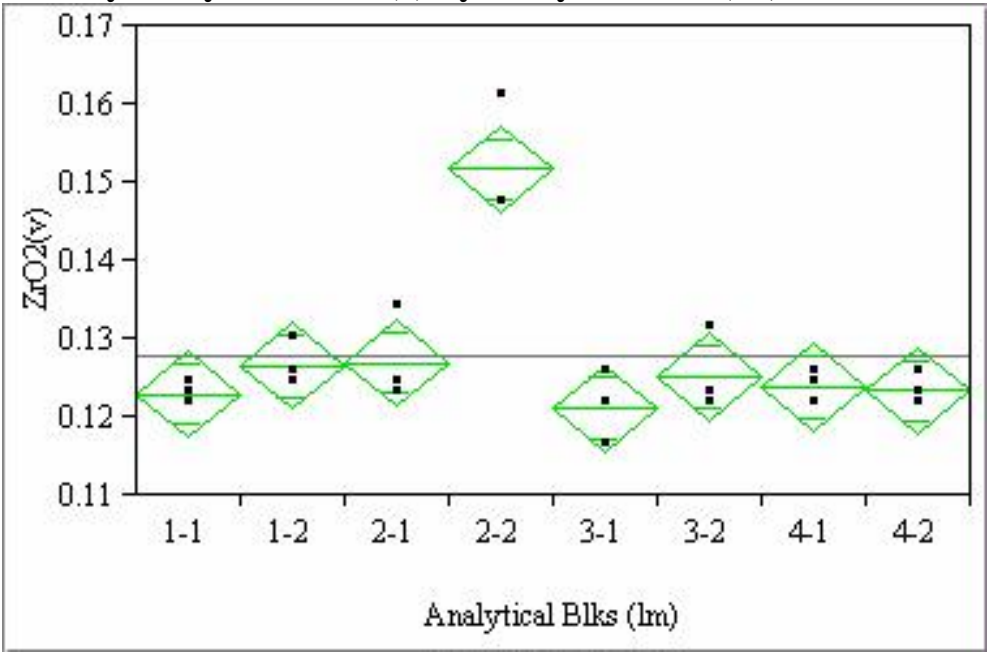

Oneway Anova

Adj Rsquare

0.863702

0.804071

0.004505

Mean of Response

Observations (or Sum Wgts)

Source $\quad$ DF Sum of Squares Mean Square F Ratio Prob > F

$\begin{array}{lrrrrr}\text { Analytical Blks (lm) } & 7 & 0.00205814 & 0.000294 & 14.4842 & <.0001\end{array}$

$\begin{array}{lrrr}\text { Analytical Blks (lm) } & 7 & 0.00205814 & 0.000294 \\ \text { Error } & 16 & 0.00032479 & 0.000020 \\ \text { C. Total } & 23 & 0.00238293 & \end{array}$

Means for Oneway Anova

Level Number Mean Std Error Lower 95\% Upper 95\%

$\begin{array}{llllll}1-1 & 3 & 0.122923 & 0.00260 & 0.11741 & 0.12844\end{array}$

$\begin{array}{llllll}1-2 & 3 & 0.126525 & 0.00260 & 0.12101 & 0.13204\end{array}$

$\begin{array}{llllll}2-1 & 3 & 0.126975 & 0.00260 & 0.12146 & 0.13249 \\ 2-2 & 3 & 0.151740 & 0.00260 & 0.14623 & 0.15725\end{array}$

$\begin{array}{llllll}3-1 & 3 & 0.151740 & 0.00260 & 0.14623 & 0.15725 \\ 3-2 & 3 & 0.121122 & 0.00260 & 0.11561 & 0.12664\end{array}$

$\begin{array}{llllll}3-2 & 3 & 0.125174 & 0.00260 & 0.11966 & 0.12669 \\ 4 & 3 & 0.123823 & 0.00260 & 0.1831 & 0.12934\end{array}$

$\begin{array}{llllll}4-1 & 3 & 0.123823 & 0.00260 & 0.11831 & 0.12934 \\ 4 & 3 & 0.123373 & 0.00260 & 0.11786 & 0.12889\end{array}$

Std Error uses a pooled estimate of error variance
Oneway Analysis of ZrO2(v) By Analytical Blks (lm)

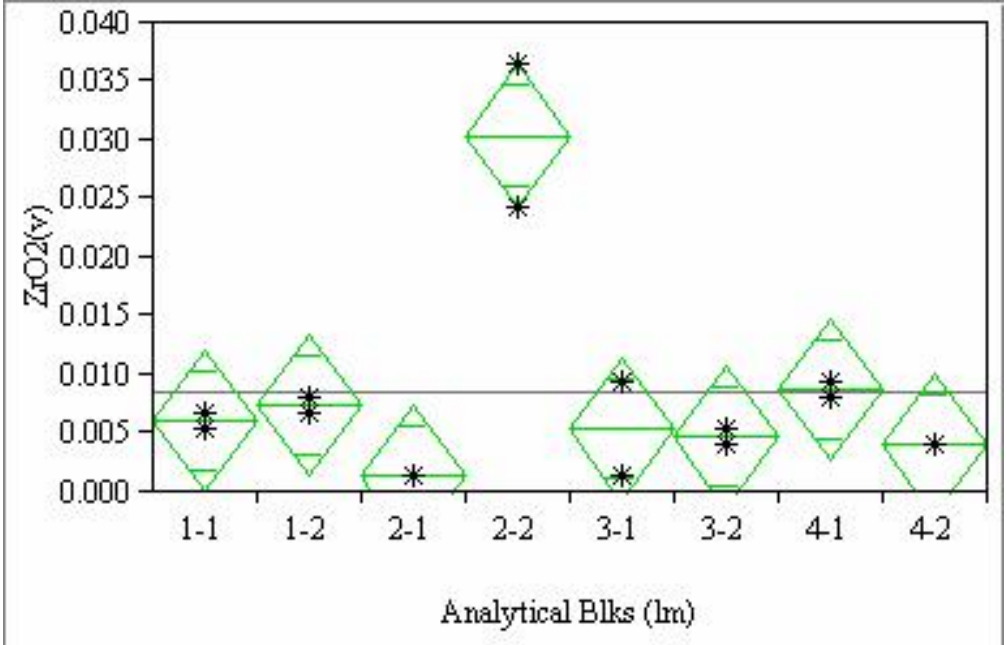

\begin{tabular}{lrrrrr}
\hline $\begin{array}{l}\text { Oneway Anova } \\
\text { Summary of Fit }\end{array}$ & & & & & \\
Rsquare & 0.913254 & & & \\
Adj Rsquare & 0.837351 & & & \\
$\begin{array}{l}\text { Root Mean Square Error } \\
\text { Mean of Response }\end{array}$ & 0.003715 & & & & \\
$\begin{array}{l}\text { Observations (or Sum Wgts) } \\
\text { Analysis of Variance }\end{array}$ & 0.008527 & 16 & & & \\
Source & DF & Sum of Squares & Mean Square & F Ratio & Prob > F \\
Analytical Blks (lm) & 7 & 0.00116219 & 0.000166 & 12.0319 & 0.0011 \\
Error & 8 & 0.00011039 & 0.000014 & & \\
C. Total & 15 & 0.00127259 & & &
\end{tabular}

C. Total

Oneway Anova

Level Number Mean Std Error Lower 95\% Upper 95\%

$\begin{array}{llllll}1-1 & 2 & 0.006079 & 0.00263 & 0.00002 & 0.01214 \\ 1-2 & 2 & 0.007429 & 0.00263 & 0.00137 & 0.01349\end{array}$

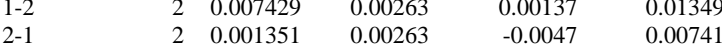

$\begin{array}{llllll}1-2 & 2 & 0.001351 & 0.00263 & -0.0047 & 0.00741 \\ 2-2 & 2 & 0.030393 & 0.00263 & 0.02434 & 0.03645 \\ 3-1 & 2 & 0.005403 & 0.00263 & -0.0007 & 0.01146\end{array}$

$\begin{array}{llllll}3-1 & 2 & 0.005403 & 0.00263 & -0.0007 & 0.01146 \\ 3-2 & 2 & 0.004728 & 0.00263 & -0.0013 & 0.01078\end{array}$

$\begin{array}{rrrrrr}3-2 & 2 & 0.004728 & 0.00263 & -0.0013 & 0.01078 \\ 4-1 & 2 & 0.008780 & 0.00263 & 0.00272 & 0.01484 \\ 4-2 & 2 & 0.004052 & 0.00263 & -0.002 & 0.01011\end{array}$

Std Error uses a pooled estimate of error variance 
Exhibit E4. Measurements of Standards Prepared Using the Peroxide Fusion (PF) Method by Oxide by Analytical Block (Small Square - Batch 1 and Asterisk - Ustd)

Oneway Analysis of A12O3 (v) By Analytical Block

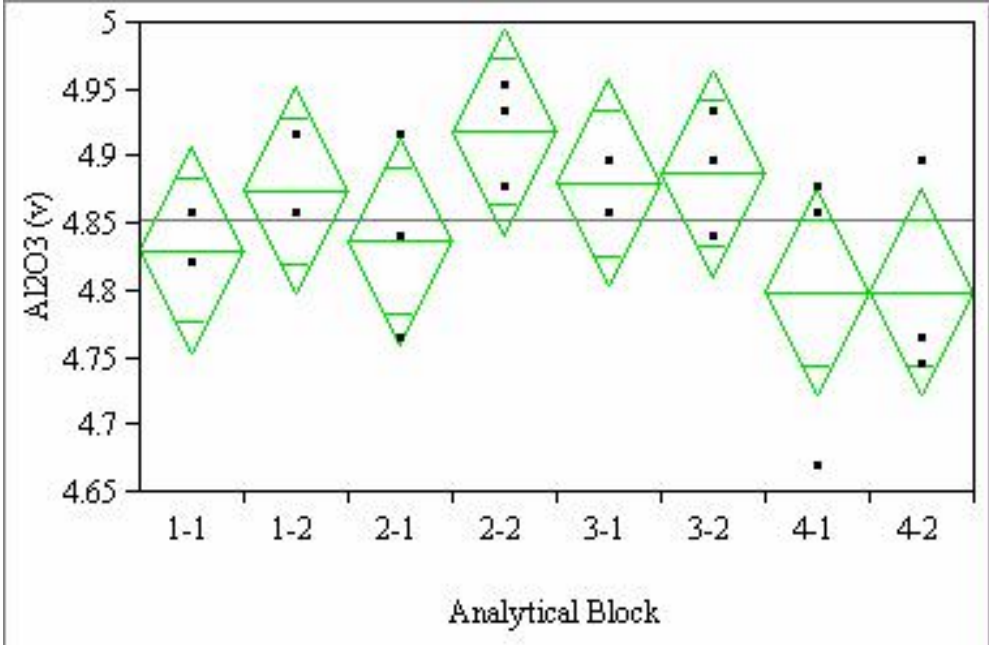

\section{Oneway Anova}

Rsquare

Adj Rsquare

Root Mean Square Ertor

Mean of Response

0.1201

0.062786

Observations (or Sum Wgts)

Source DF Sum of Squares Mean Square F Ratio Prob > F

$\begin{array}{lrlrrr}\text { Analytical Block } & 7 & 0.03997148 & 0.005710 & 1.4485 & 0.2540\end{array}$

Error $\quad 16 \quad 0.06307371$

$\begin{array}{lll}\text { C. Total } & 23 & 0.10304519\end{array}$

Level Number Mean Std Error Lower 95\% Upper 95\%

$\begin{array}{rrrrrr}1-1 & 3 & 4.83082 & 0.03625 & 4.7540 & 4.9077 \\ 1-2 & & 3 & 4.87491 & 0.03625 & 4.7981\end{array}$

$\begin{array}{llllll}1-2 & 3 & 4.87491 & 0.03625 & 4.7981 & 4.9518 \\ 2-1 & 3 & 4.83712 & 0.03625 & 4.7603 & 4.9140\end{array}$

$\begin{array}{llllll}2-2 & 3 & 4.91900 & 0.03625 & 4.8422 & 4.9958\end{array}$

$\begin{array}{llllll}3-1 & 3 & 4.88121 & 0.03625 & 4.8044 & 4.9581\end{array}$

$\begin{array}{llllll}3-2 & 3 & 4.88751 & 0.03625 & 4.8107 & 4.9644 \\ 4-1 & 3 & 4.79933 & 0.03625 & 4.7225 & 4.8762\end{array}$

$\begin{array}{llllll}4-1 & 3 & 4.79933 & 0.03625 & 4.7225 & 4.8762 \\ 4-2 & 3 & 4.79933 & 0.03625 & 4.7225 & 4.8762\end{array}$

Std Error uses a pooled estimate of error variance
Oneway Analysis of Al2O3 (v) By Analytical Block

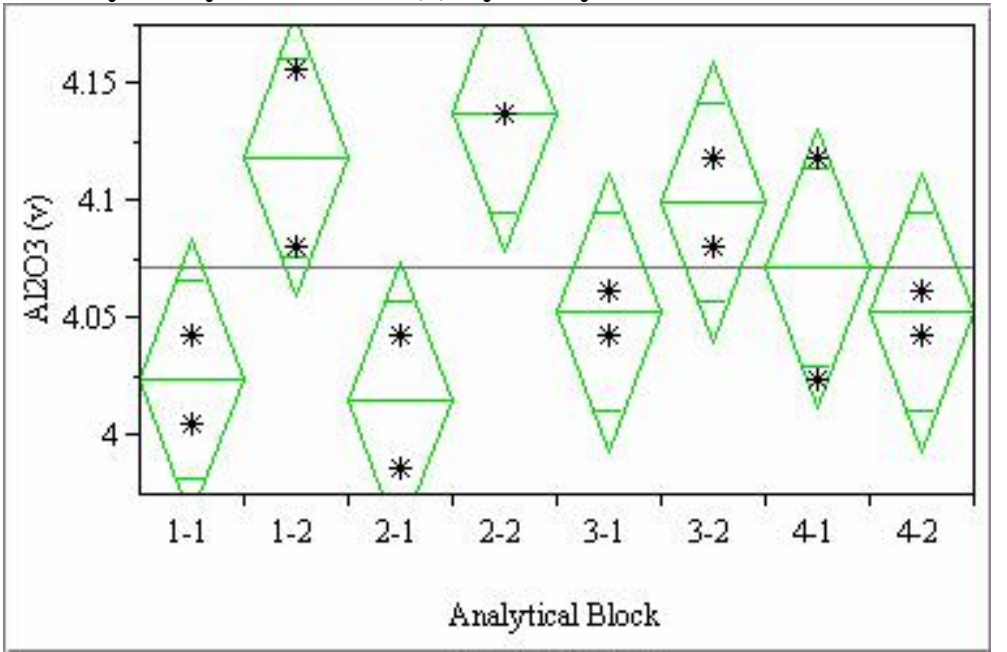

\section{Oneway Anova
Summary of Fit}

Rsquare

Root Mean Square Error $\quad 0.46934$

Mean of Response $\quad 0.03659$

Observations (or Sum Wots) $\quad 4.071872$

Analysis of Variance

$\begin{array}{lllll}\text { Source DF Sum of Squares Mean Square } & \text { F Ratio Prob }>\text { F }\end{array}$

$\begin{array}{lrrrrr}\text { Analytical Block } & 7 & 0.02713360 & 0.003876 & 2.8952 & 0.0798\end{array}$

$\begin{array}{llll}\text { Error } & 8 & 0.01071063 & 0.001339\end{array}$

C. Total $15-0.03784423$

Means for Oneway Anova

Level Number Mean Std Error Lower 95\% Upper 95\%

$\begin{array}{lrrrrr}1-1 & 2 & 4.02464 & 0.02587 & 3.9650 & 4.0843\end{array}$

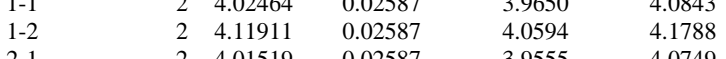

$\begin{array}{llllll}2-1 & 2 & 4.01519 & 0.02587 & 3.9555 & 4.0749 \\ 2-2 & 2 & 4.13800 & 0.02587 & 4.0783 & 4.1977\end{array}$

$\begin{array}{llllll}2-2 & 2 & 4.13800 & 0.02587 & 4.0783 & 4.1977 \\ 3-1 & 2 & 4.05298 & 0.02587 & 3.9933 & 4.1126 \\ 3-2 & 2 & 4.10022 & 0.02587 & 4.0406 & 4.1599\end{array}$

$\begin{array}{llllll}3-2 & 2 & 4.10022 & 0.02587 & 4.0406 & 4.1599 \\ 4-1 & 2 & 4.07187 & 0.02587 & 4.0122 & 4.1315\end{array}$

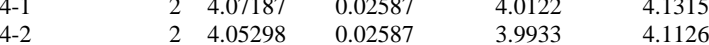

Std Error uses a pooled estimate of error variance 
Exhibit E4. Measurements of Standards Prepared Using the Peroxide Fusion (PF) Method by Oxide by Analytical Block

(Small Square - Batch 1 and Asterisk - Ustd)

Oneway Analysis of B2O3(v) By Analytical Block

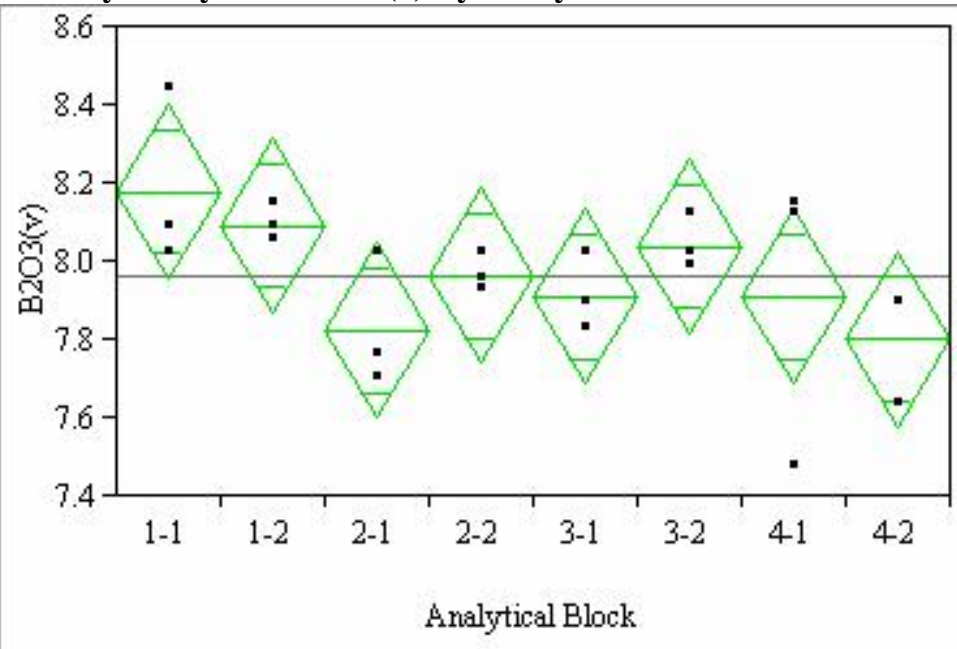

Oneway Anova Summary of Fit

Rsquare

dj Rsquare

Root Mean Square Error

Mean of Response

0.402065

0.140468

0.1825

Observations (or Sum Wgts)

Analysis of Variance

$\begin{array}{lrrrrr}\text { Source } & \text { DF } & \text { Sum of Squares } & \text { Mean Square } & \text { F Ratio } & \text { Prob > F } \\ \text { Analytical Block } & 7 & 0.35833557 & 0.051191 & 1.5370 & 0.2248\end{array}$

$\begin{array}{lll}0.051191 & 1.5370 & 0.2248 \\ 0.033306 & & \end{array}$

Eror

$23 \quad 0.89123823$

Means for Oneway Anova

Level Number Mean Std Error Lower 95\% Upper 95\%

$\begin{array}{llllrr}1-1 & 3 & 8.17855 & 0.10537 & 7.9552 & 8.4019\end{array}$

$\begin{array}{llllll}1-2 & 3 & 8.09268 & 0.10537 & 7.8693 & 8.3160\end{array}$

$\begin{array}{llllll}2-1 & 3 & 7.82436 & 0.10537 & 7.6010 & 8.0477 \\ 2-2 & 3 & 7.96389 & 0.10537 & 7.7405 & 8.1873\end{array}$

$\begin{array}{llllll}3-1 & 3 & 7.91022 & 0.10537 & 7.6869 & 8.1336\end{array}$

$\begin{array}{llllll}3-2 & 3 & 8.03902 & 0.10537 & 7.8156 & 8.2624 \\ 4-1 & 3 & 7.91022 & 0.10537 & 7.6869 & 8.1336\end{array}$

$\begin{array}{llllll}4-2 & 3 & 7.80289 & 0.10537 & 7.5795 & 8.0263\end{array}$

Std Error uses a pooled estimate of error variance
Oneway Analysis of B2O3(v) By Analytical Block

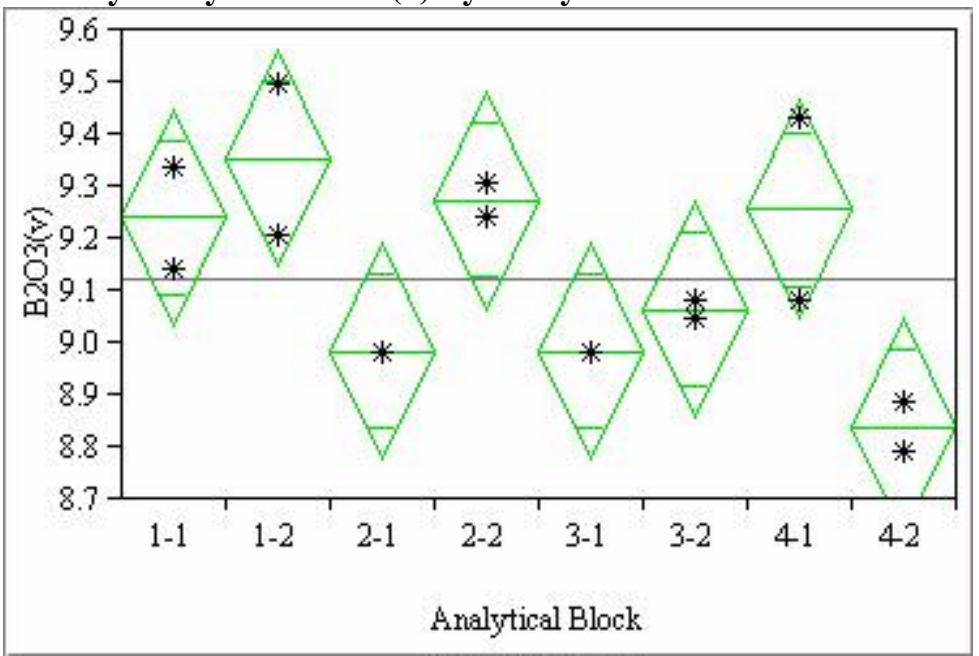

Oneway Anova

Summary of

$\begin{array}{ll}\text { Rsquare } & 0.779624 \\ \text { Adj Rsquare } & 0.586795\end{array}$

0.779624

$\begin{array}{ll}\text { Root Mean Square Error } & 0.127786 \\ \text { Mean of Response } & 9.124392\end{array}$

Observations (or Sum Wgts) $\quad 16$

Analysis of Variance

Source DF Sum of Squares Mean Square F Ratio Prob > F

$\begin{array}{lrrrrr}\text { Analytical Block } & 7 & 0.46214272 & 0.066020 & 4.0431 & 0.0342 \\ \text { Error } & 8 & 0.13063373 & 0.016329 & & \end{array}$

Error

0.01632

C. Total

$15 \quad 0.59277645$

Means for Oneway Anova

$\begin{array}{lrrrrr}\text { Level } & \text { Number } & \text { Mean } & \text { Std Error } & \text { Lower 95\% } & \text { Upper 95\% } \\ 1-1 & 2 & 9.24111 & 0.09036 & 9.0327 & 9.4495\end{array}$

$\begin{array}{llllll}1-2 & 2 & 9.35381 & 0.09036 & 9.1454 & 9.5622 \\ 2-1 & 2 & 8.98352 & 0.09036 & 8.7752 & 9.1919\end{array}$

$\begin{array}{llllll}2-2 & 2 & 9.27331 & 0.09036 & 9.0649 & 9.4817\end{array}$

$\begin{array}{llllll}3-1 & 2 & 8.98352 & 0.09036 & 8.7752 & 9.1919 \\ 3-2 & 2 & 9.06402 & 0.09036 & 8.8557 & 9.2724\end{array}$

$\begin{array}{llllll}4-1 & 2 & 9.25721 & 0.09036 & 9.0488 & 9.4656 \\ 4-2 & 2 & 8.83863 & 0.09036 & 8.6303 & 9.0470\end{array}$

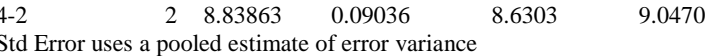


Exhibit E4. Measurements of Standards Prepared Using the Peroxide Fusion (PF) Method by Oxide by Analytical Block

(Small Square - Batch 1 and Asterisk - Ustd)

Oneway Analysis of Li2O(v) By Analytical Block

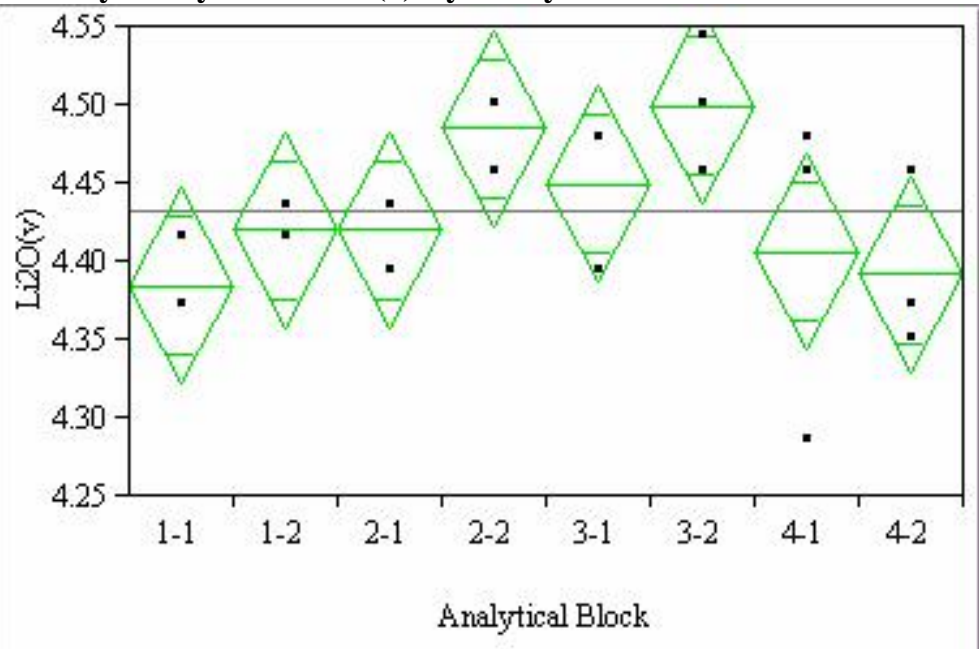

Oneway Anova

Summary of Fit

Rsquare

Adj Rsquare

Root Mean Square Error

Mean of Response

Observations (or Sum Wgts)

Analysis of Variance

$\begin{array}{lrrrrr}\text { Source } & \text { DF } & \text { Sum of Squares } & \text { Mean Square } & \text { F Ratio } & \text { Prob > F } \\ \text { Analytical Block } & 7 & 0.03736951 & 0.005339 & 2.0476 & 0.1114 \\ \text { Error } & 16 & 0.04171481 & 0.002607 & & \end{array}$

$\begin{array}{ll}16 & 0.0417148 \\ 23 & 0.07908432\end{array}$

Means for Oneway Anova

$\begin{array}{lrrrrr}\text { Level } & \text { Number } & \text { Mean } & \text { Std Error } & \text { Lower 95\% } & \text { Upper 95\% } \\ 1-1 & 3 & 4.38474 & 0.02948 & 4.3222 & 4.4472\end{array}$

$\begin{array}{llllll}1-2 & 3 & 4.42062 & 0.02948 & 4.3581 & 4.4831\end{array}$

$\begin{array}{llllll}2-1 & 3 & 4.42062 & 0.02948 & 4.3581 & 4.4831 \\ 2-2 & 3 & 4.48521 & 0.02948 & 4.4227 & 4.5477\end{array}$

$\begin{array}{llllll}3-1 & 3 & 4.44933 & 0.02948 & 4.3868 & 4.5118\end{array}$

$\begin{array}{llllll}3-2 & 3 & 4.49956 & 0.02948 & 4.4371 & 4.5621\end{array}$

$\begin{array}{llllll}4-1 & 3 & 4.40627 & 0.02948 & 4.3438 & 4.4688\end{array}$

$3 \quad 4.39192 \quad 0.02948$

4.3294

4.4544
Oneway Analysis of Li2O(v) By Analytical Block

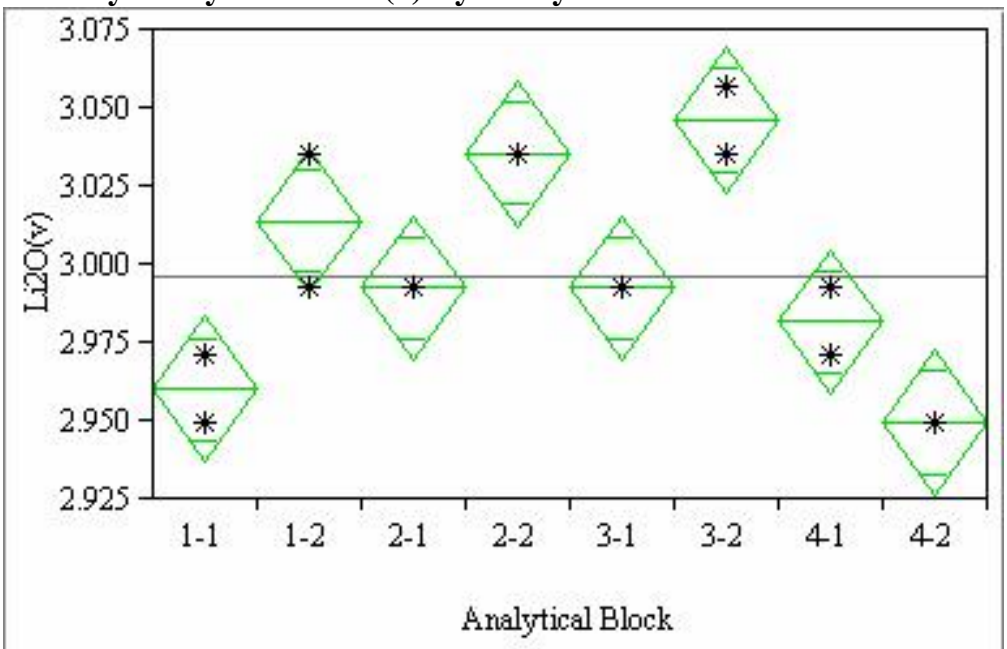

Oneway Anova
Summary of Fit

Rsquare

0.908943

Adj Rsquare $\quad 0.829268$

$\begin{array}{lr}\text { Root Mean Square Error } & 0.01424 \\ \text { Mean of Response } & 2.996568\end{array}$

Observations (or Sum Wgts) $\quad 16$

Analysis of Variance

Source DF Sum of Squares Mean Square F Ratio Prob $>$ F

$\begin{array}{lrlrrr}\text { Analytical Block } & 7 & 0.01619346 & 0.002313 & 11.4082 & 0.0013\end{array}$

$\begin{array}{lrrr}\text { Error } & 8 & 0.00162224 & 0.000203 \\ \text { C. Total } & 15 & 0.01781570 & \end{array}$

Means for Oneway Anova

Level Number Mean Std Error Lower 95\% Upper 95\%

$\begin{array}{rrrrrr}1-1 & 2 & 2.96024 & 0.01007 & 2.9370 & 2.9835 \\ 1-2 & 2 & 3.01406 & 0.01007 & 2.9908 & 3.03758\end{array}$

$\begin{array}{llllll}1-2 & 2 & 3.01406 & 0.01007 & 2.9908 & 3.0373 \\ 2-1 & 2 & 2.99253 & 0.01007 & 2.9693 & 3.0158\end{array}$

$\begin{array}{llllll}2-2 & 2 & 3.03559 & 0.01007 & 3.0124 & 3.0158 \\ 3-1 & 2 & 2.99253 & 0.01007 & 2.9693 & 3.0158\end{array}$

$\begin{array}{llllll}3-1 & 2 & 2.99253 & 0.01007 & 2.9693 & 3.0158 \\ 3-2 & 2 & 3.04635 & 0.01007 & 3.0231 & 3.0696\end{array}$

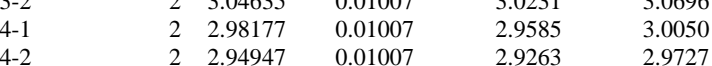

$\begin{array}{llll}\text { Std Error uses a pooled estimate of error variance } & 2.9263 & 2.9727\end{array}$ 
Exhibit E4. Measurements of Standards Prepared Using the Peroxide Fusion (PF) Method by Oxide by Analytical Block

(Small Square - Batch 1 and Asterisk - Ustd)

Oneway Analysis of U308(v) By Analytical Block

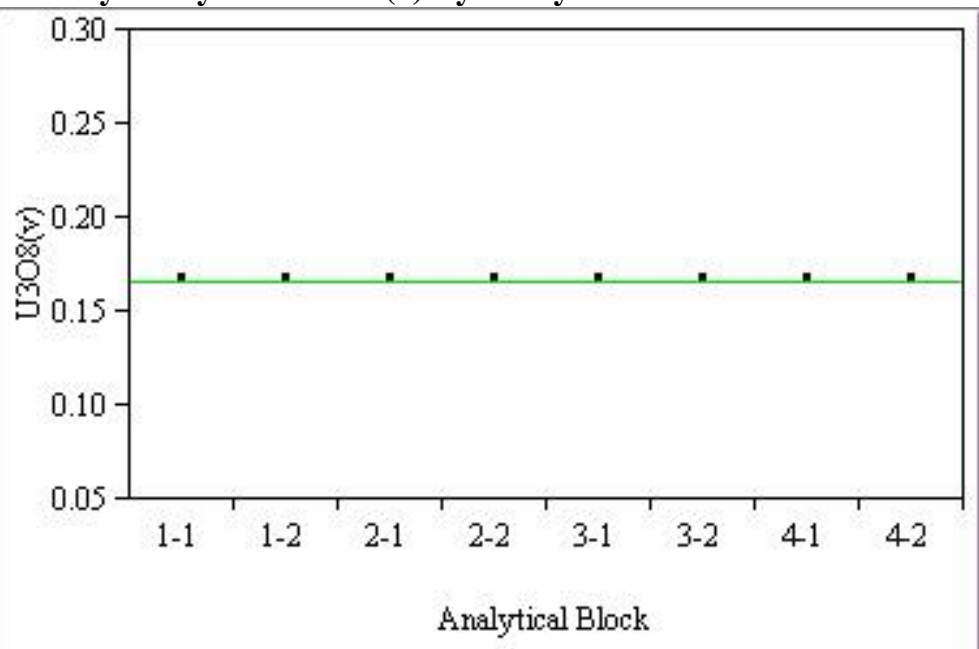

Oneway Anova

Summary of Fit

Rsquare

Root Mean Square Erro

Mean of Response

0
0.165678
24

Analysis of Variance

DF
7

Analytical Block

7 23

C. Total

Means for Oneway Anova

$\begin{array}{lrrrrr}\text { Level } & \text { Number } & \text { Mean } & \text { Std Error } & \text { Lower 95\% } & \text { Upper 95\% } \\ 1-1 & 3 & 0.165678 & 0 & 0.16568 & 0.16568 \\ 1-2 & 3 & 0.165678 & 0 & 0.16568 & 0.16568 \\ 2-1 & 3 & 0.165678 & 0 & 0.16568 & 0.16568 \\ 2-2 & 3 & 0.165678 & 0 & 0.16568 & 0.16568 \\ 3-1 & 3 & 0.165678 & 0 & 0.16568 & 0.16568 \\ 3-2 & 3 & 0.165678 & 0 & 0.16568 & 0.16568 \\ 4-1 & 3 & 0.165678 & 0 & 0.16568 & 0.16568 \\ 4-2 & 3 & 0.165678 & 0 & 0.16568 & 0.16568\end{array}$

Std Error uses a pooled estimate of error variance
Oneway Analysis of U308(v) By Analytical Block

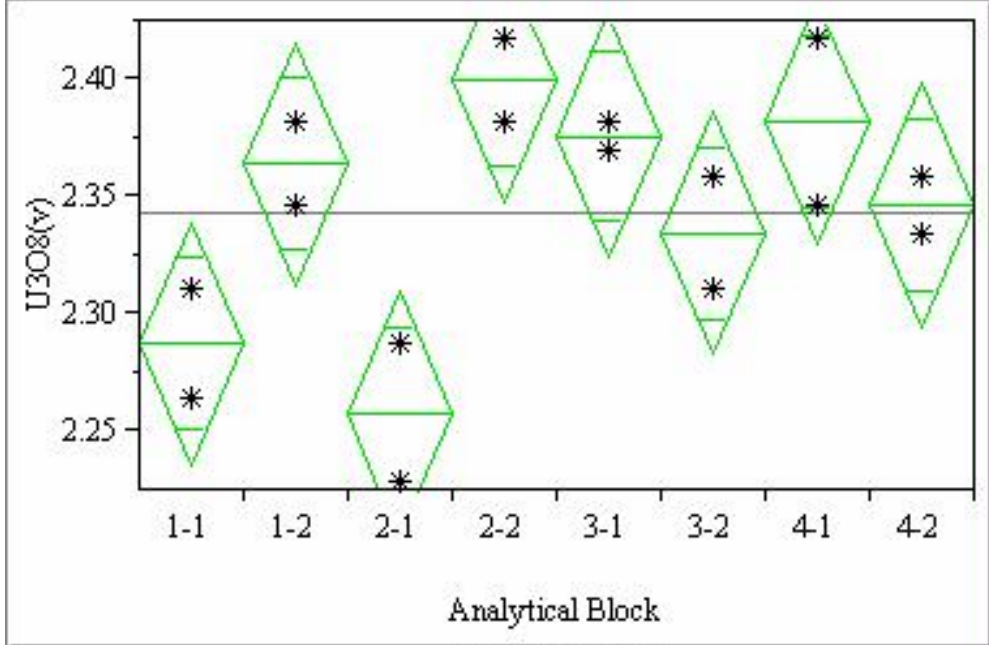

Oneway Anova

\section{Summary of}

Rsquare

Root Mean Square Error $\quad 0.03175$

$\begin{array}{lr}\text { Mean of Response } & 2.34366 \\ \text { Observations (or Sum Wgts) } & 16\end{array}$

Observations (or Sum W

DF Sum of Squares Mean Square F Ratio Prob > F

$\begin{array}{lrlrrr}\text { Analytical Block } & 7 & 0.03323325 & 0.004748 & 4.7094 & 0.0224\end{array}$

Error $\quad 8-0.00806497$

$\begin{array}{lll}\text { C. Total } & 15 & 0.04129823\end{array}$

Means for Oneway Anova

$\begin{array}{lrrrrr}\text { Level } & \text { Number } & \text { Mean } & \text { Std Error } & \text { Lower 95\% } & \text { Upper 95\% } \\ 1-1 & 2 & 2.28765 & 0.02245 & 2.2359 & 2.3394\end{array}$

$\begin{array}{llllll}1-2 & 2 & 2.36430 & 0.02245 & 2.3125 & 2.4161 \\ 2-1 & 2 & 2.25817 & 0.02245 & 2.2064 & 2.3099\end{array}$

$\begin{array}{llllll}2-2 & 2 & 2.39967 & 0.02245 & 2.3479 & 2.4514 \\ 3-1 & 2 & 2.37609 & 0.02245 & 2.3243 & 2.4279\end{array}$

$\begin{array}{llllll}3-1 & 2 & 2.37609 & 0.02245 & 2.3243 & 2.4279 \\ 3-2 & 2 & 2.33482 & 0.02245 & 2.2830 & 2.3866 \\ 4 & 2 & 2.38198 & 0.02245 & 2.3302 & 2.4338\end{array}$

$\begin{array}{llllll}4-1 & 2 & 2.38198 & 0.02245 & 2.3302 & 2.4338 \\ 4-2 & 2 & 2.34661 & 0.02245 & 2.2948 & 2.3984\end{array}$

Std Error uses a pooled estimate of error variance 
Immobilization Technology Section

WSRC-TR-2001-00131

Savannah River Technology Center

Rev. 0

Westinghouse Savannah River Company

Exhibit E5. Plots of Oxide Concentrations by Shortened Glass ID for LM Method

(Concentrations in wt\%'s, Plots for both Measured and Measured Bias-Corrected (bc) are shown)

$\mathrm{CaO}(\mathrm{v})$ By Glass ID

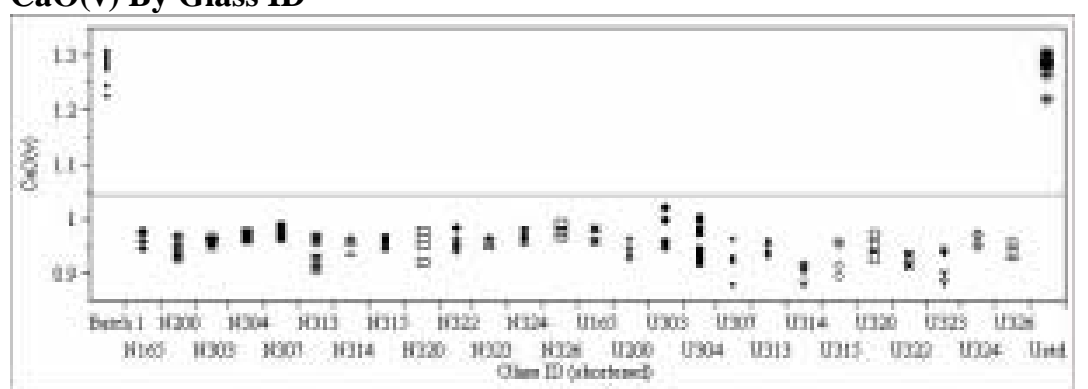

Cr2O3(v) By Glass ID

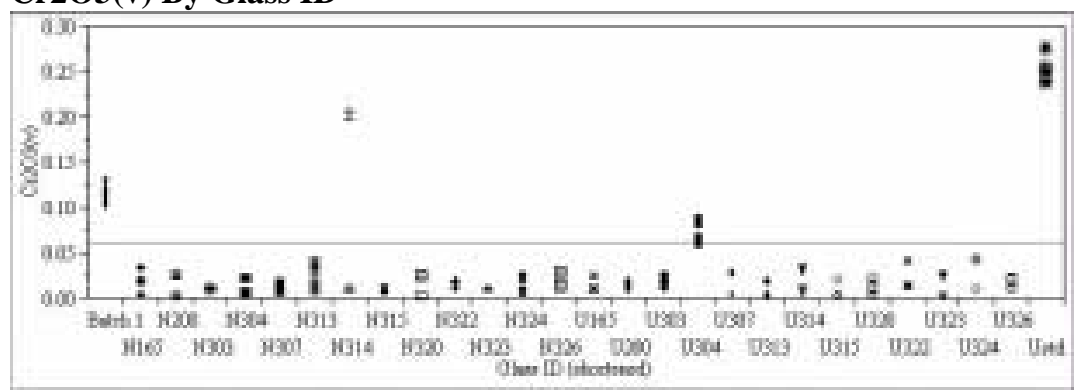

Fe203(v) By Glass ID

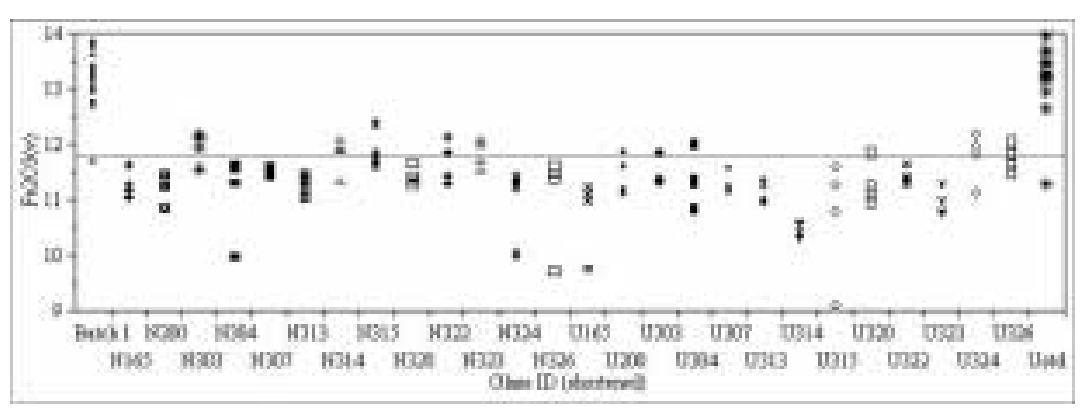

GaO be By Glass ID

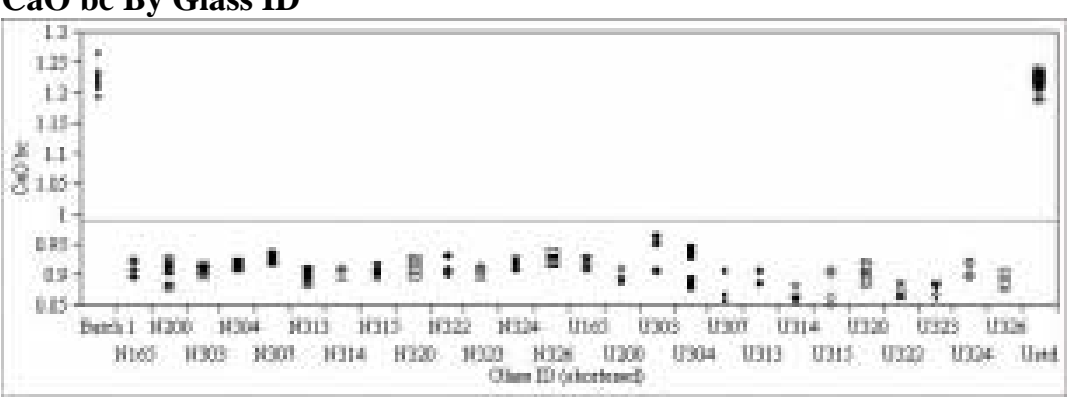

Cr2O3(v) By Glass ID

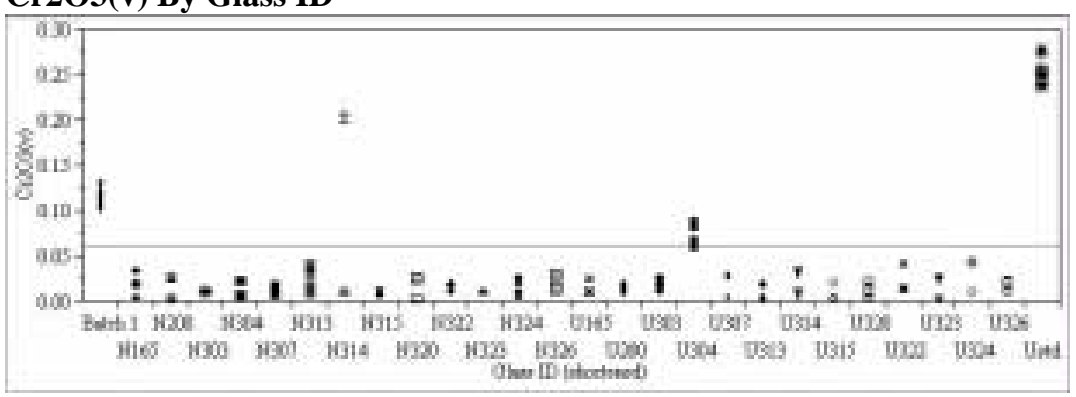

Fe2O3 bc By Glass ID

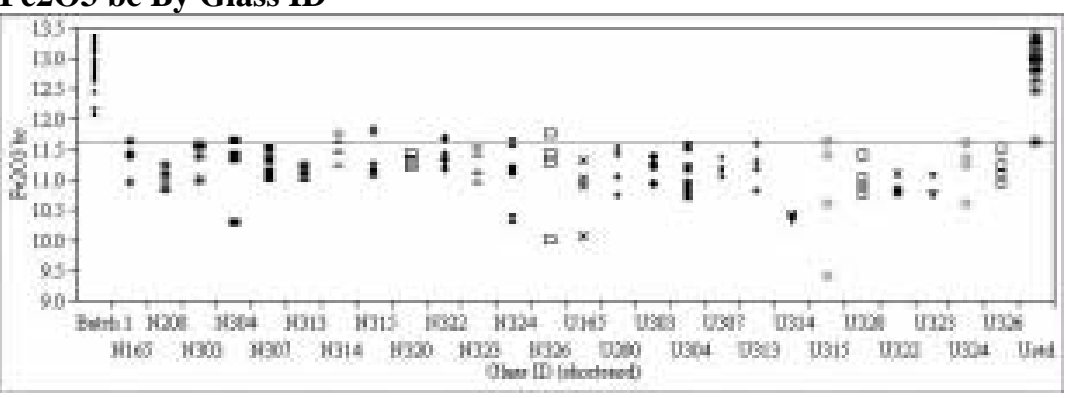

111 
Exhibit E5. Plots of Oxide Concentrations by Shortened Glass ID for LM Method (continued)

(Concentrations in wt\%'s, Plots for both Measured and Measured Bias-Corrected (bc) are shown)
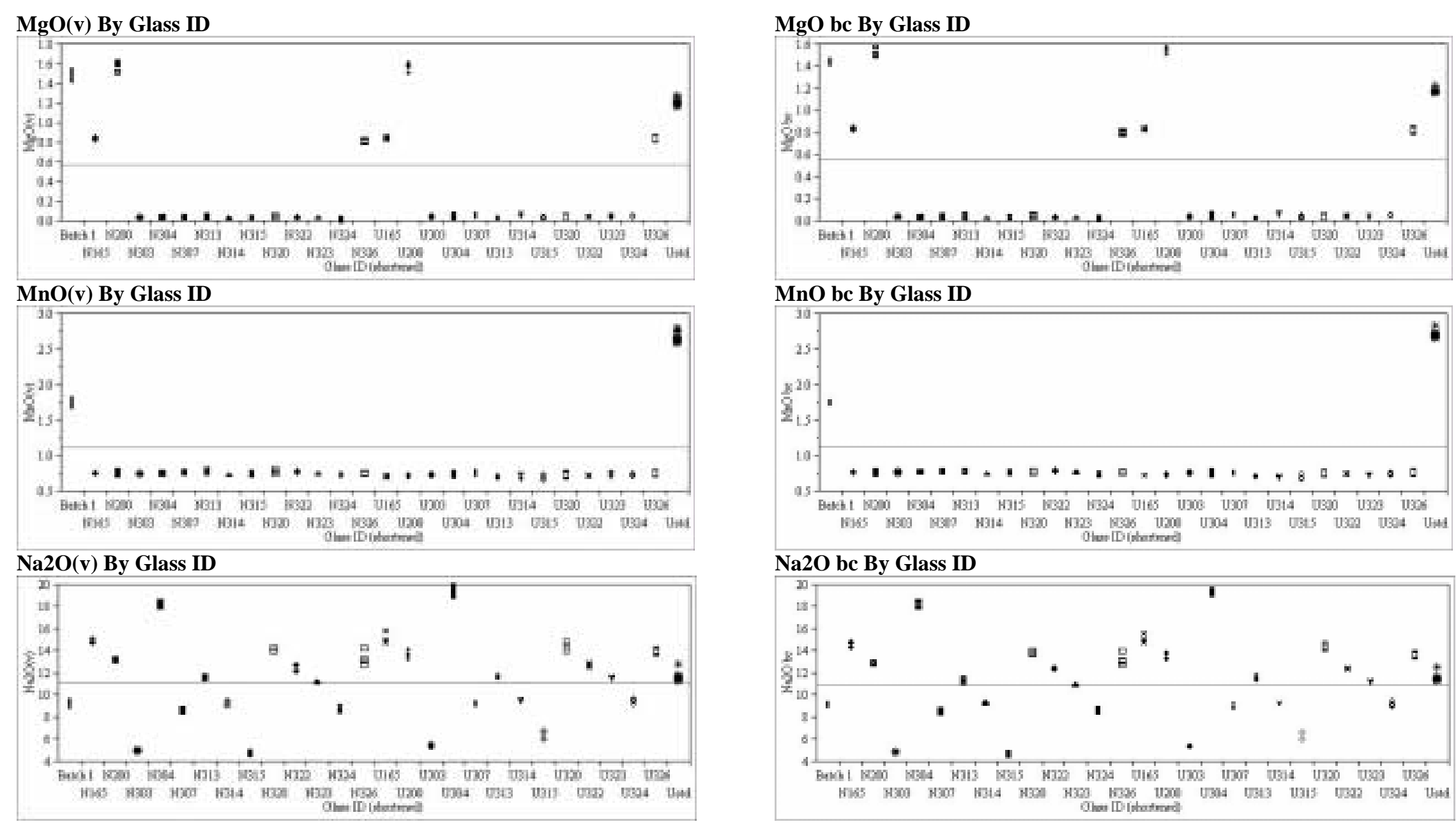
Exhibit E5. Plots of Oxide Concentrations by Shortened Glass ID for LM Method (continued)

(Concentrations in wt\%'s, Plots for both Measured and Measured Bias-Corrected (bc) are shown)

\section{$\mathrm{NiO}(v)$ By Glass ID}

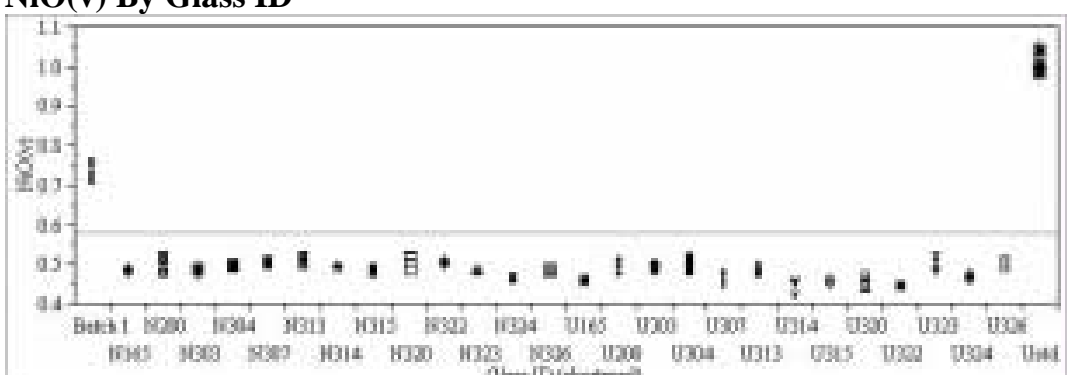

SiO2(v) By Glass ID

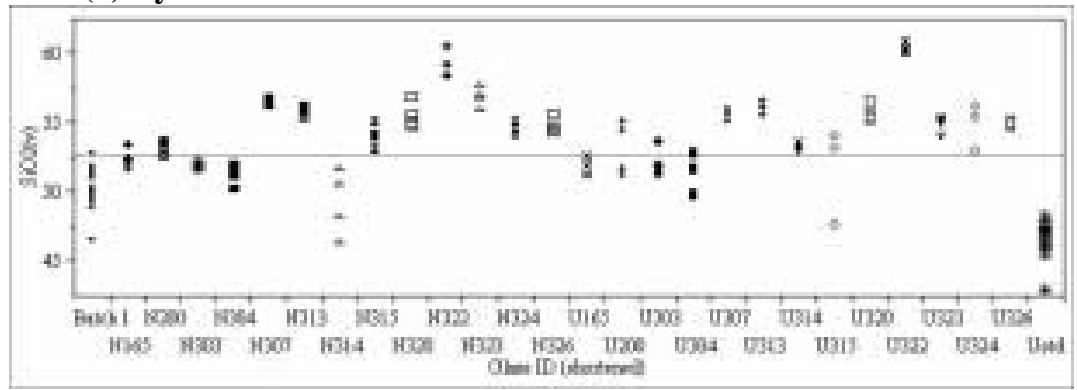

ZrO2(v) By Glass ID

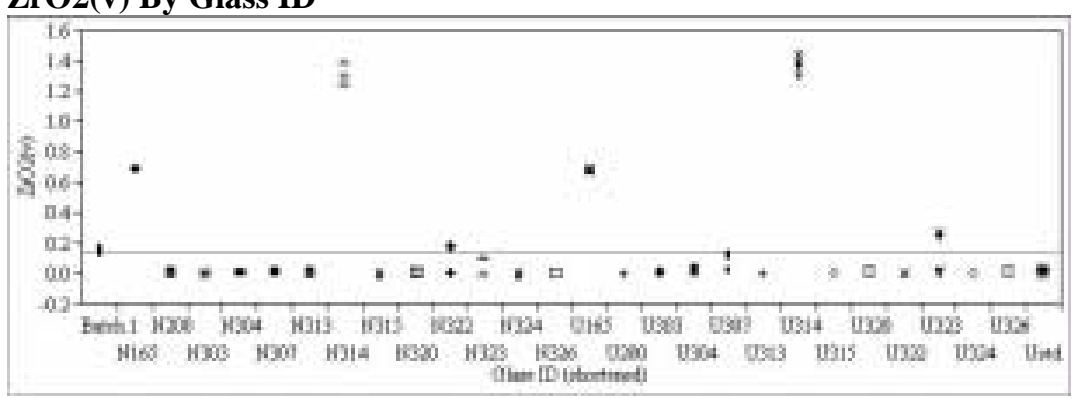

NiO be By Glass ID

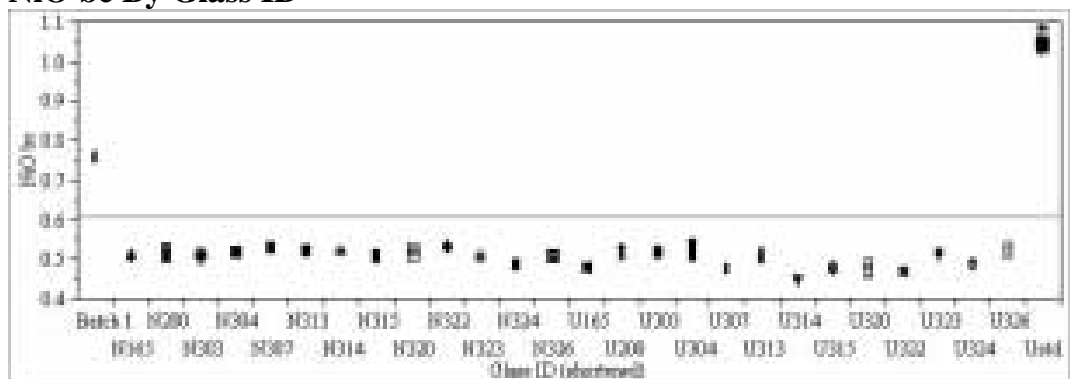

SiO2 bc By Glass ID

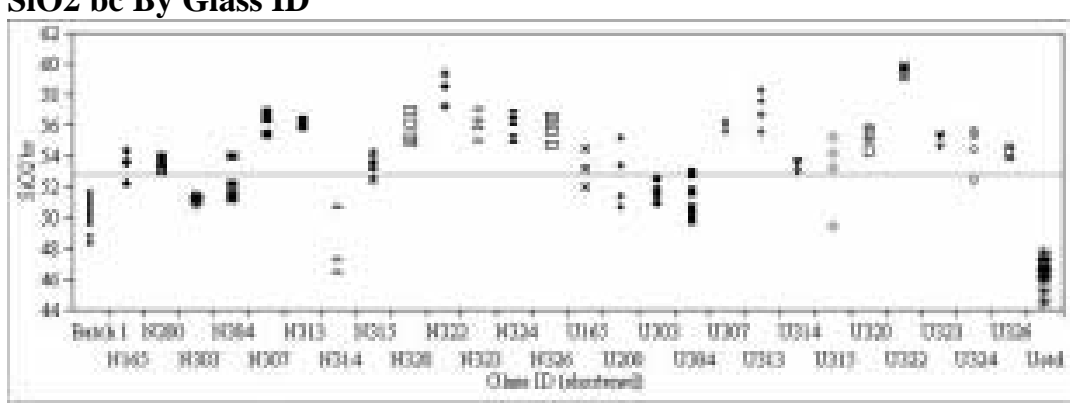

ZrO2 bc By Glass ID

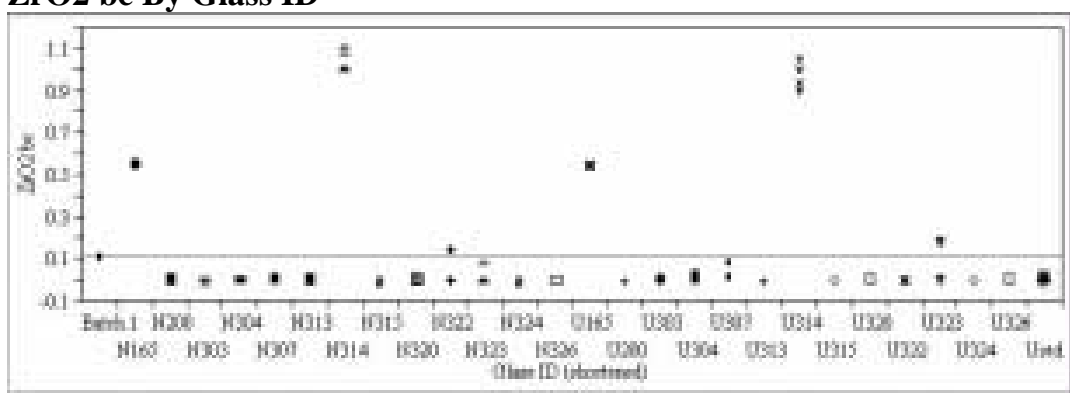


Exhibit E6. Plots of Oxide Concentrations by Shortened Glass ID for PF Method

(Concentrations in wt\%'s, Plots for both Measured and Measured Bias-Corrected (bc) are shown)

Oneway Analysis of Al2O3(v) By Glass ID

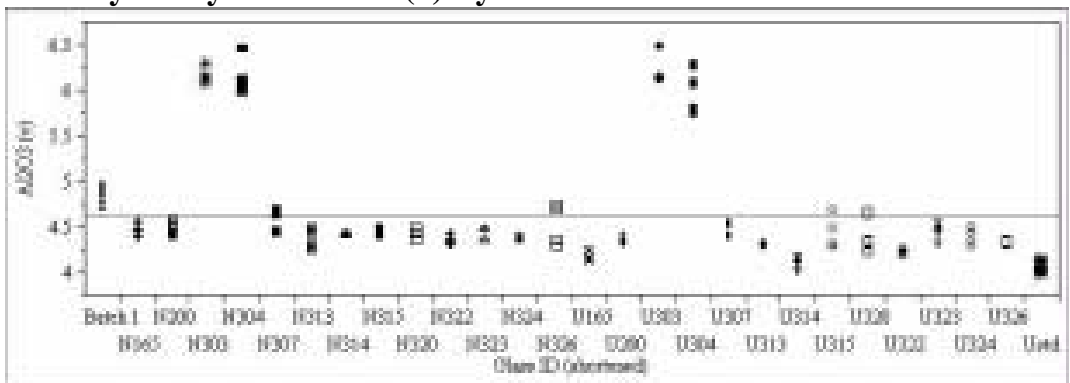

Oneway Analysis of B2O3(v) By Glass \#

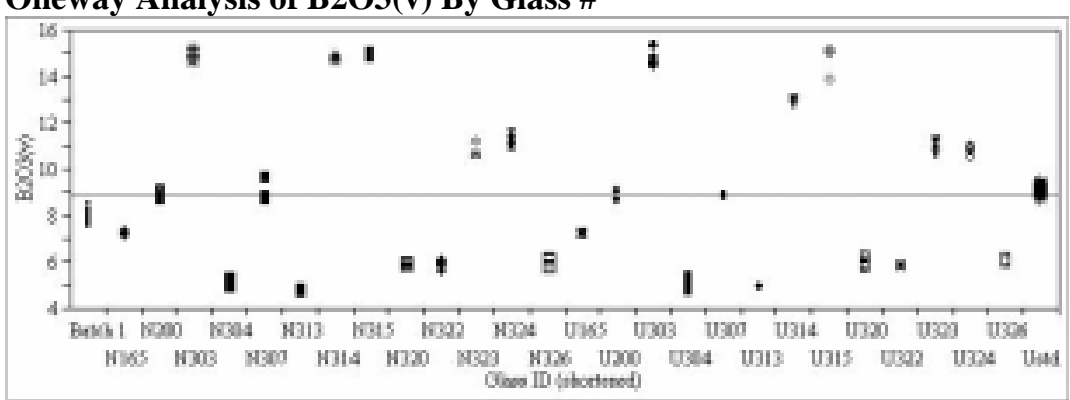

Oneway Analysis of LiO2(v) by Glass ID

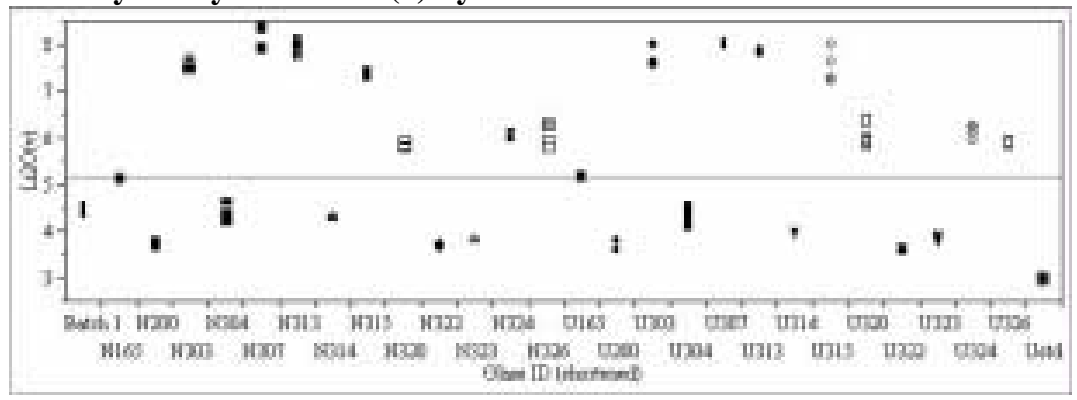

Oneway Analysis of Al2O3 bc By Glass ID

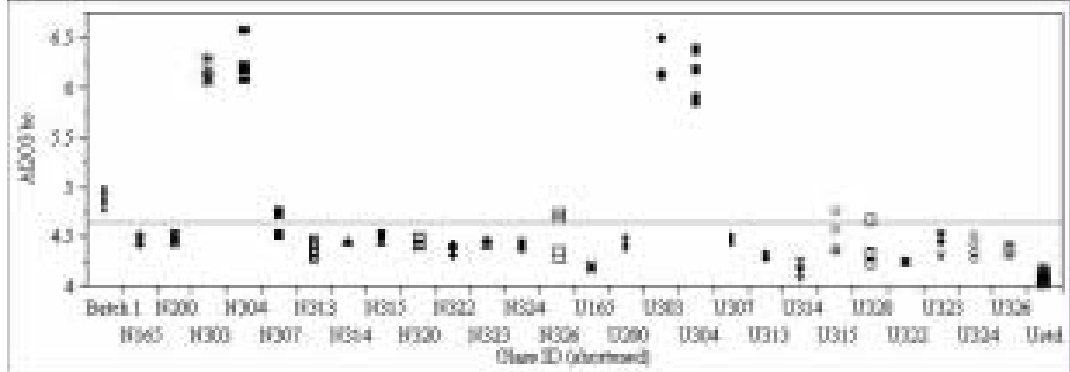

\section{Oneway Analysis of B2O3 bc By Glass \#}

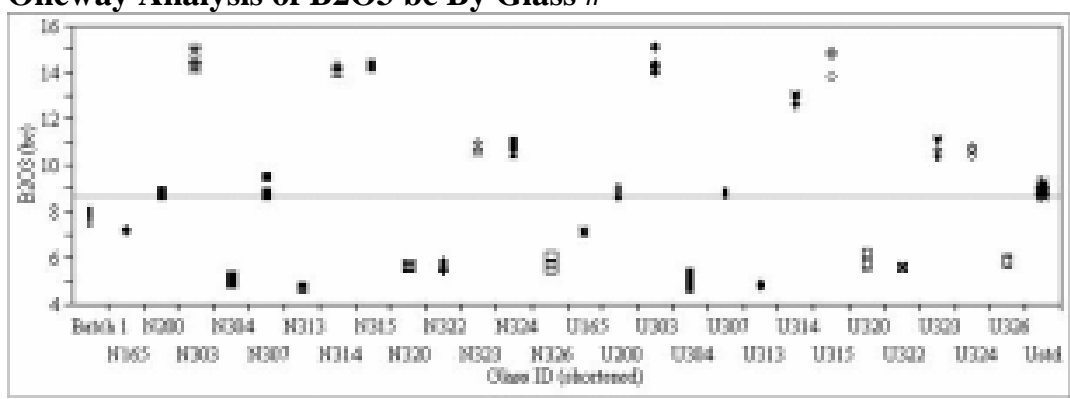

Oneway Analysis of LiO2 bc by Glass ID

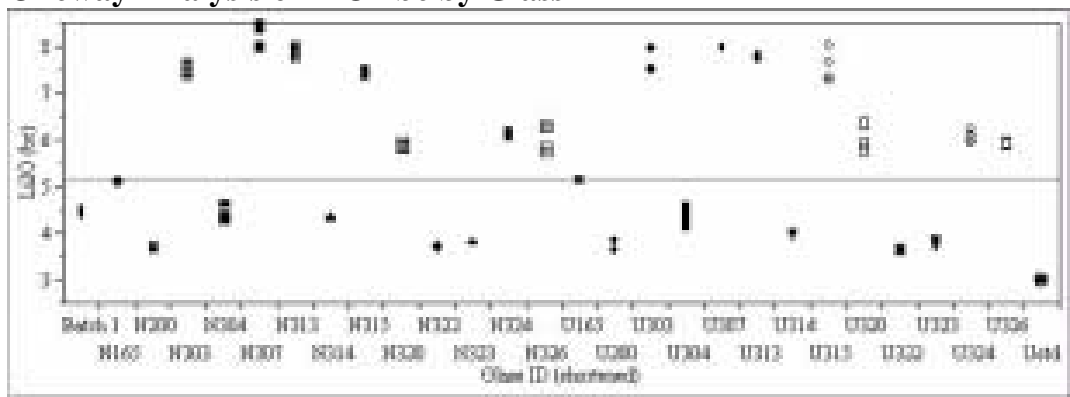


Exhibit E6. Plots of Oxide Concentrations by Shortened Glass ID for PF Method (continued) (Concentrations in wt\%'s, Plots for both Measured and Measured Bias-Corrected (bc) are shown)
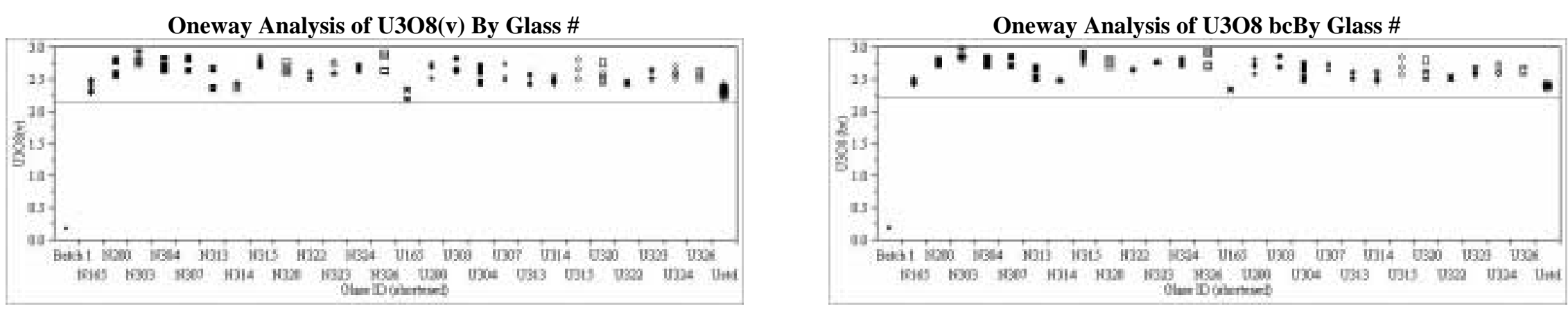
Exhibit E7. Comparisons of Measured versus Targeted Chemical Compositions (Concentrations in wt\%'s)
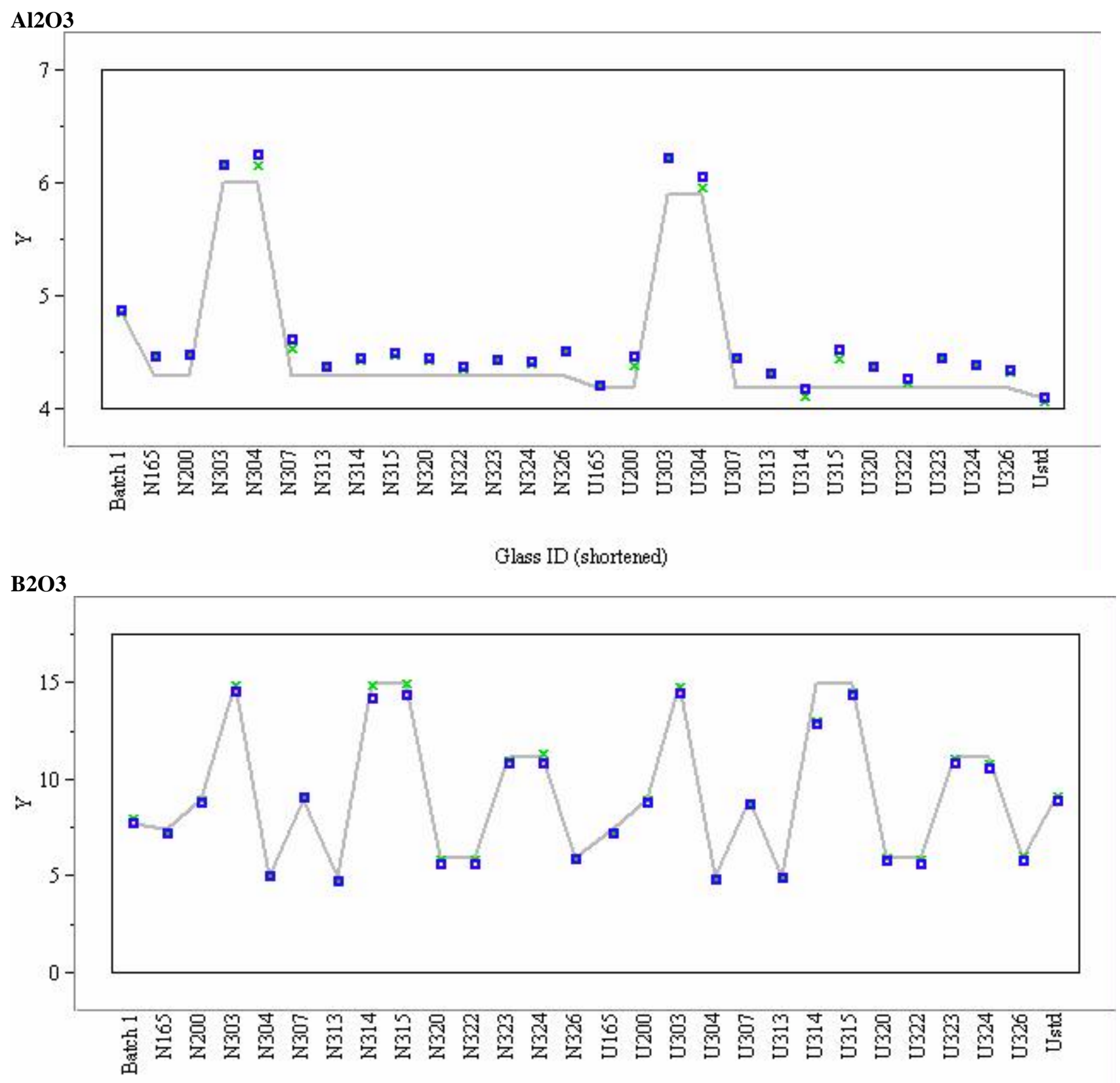

Glass ID (shortened)

$\mathrm{Y}$

- Tepos

* Merent

- Mranesta 
Exhibit E7. Comparisons of Measured versus Targeted Chemical Compositions

(Concentrations in wt\%'s)
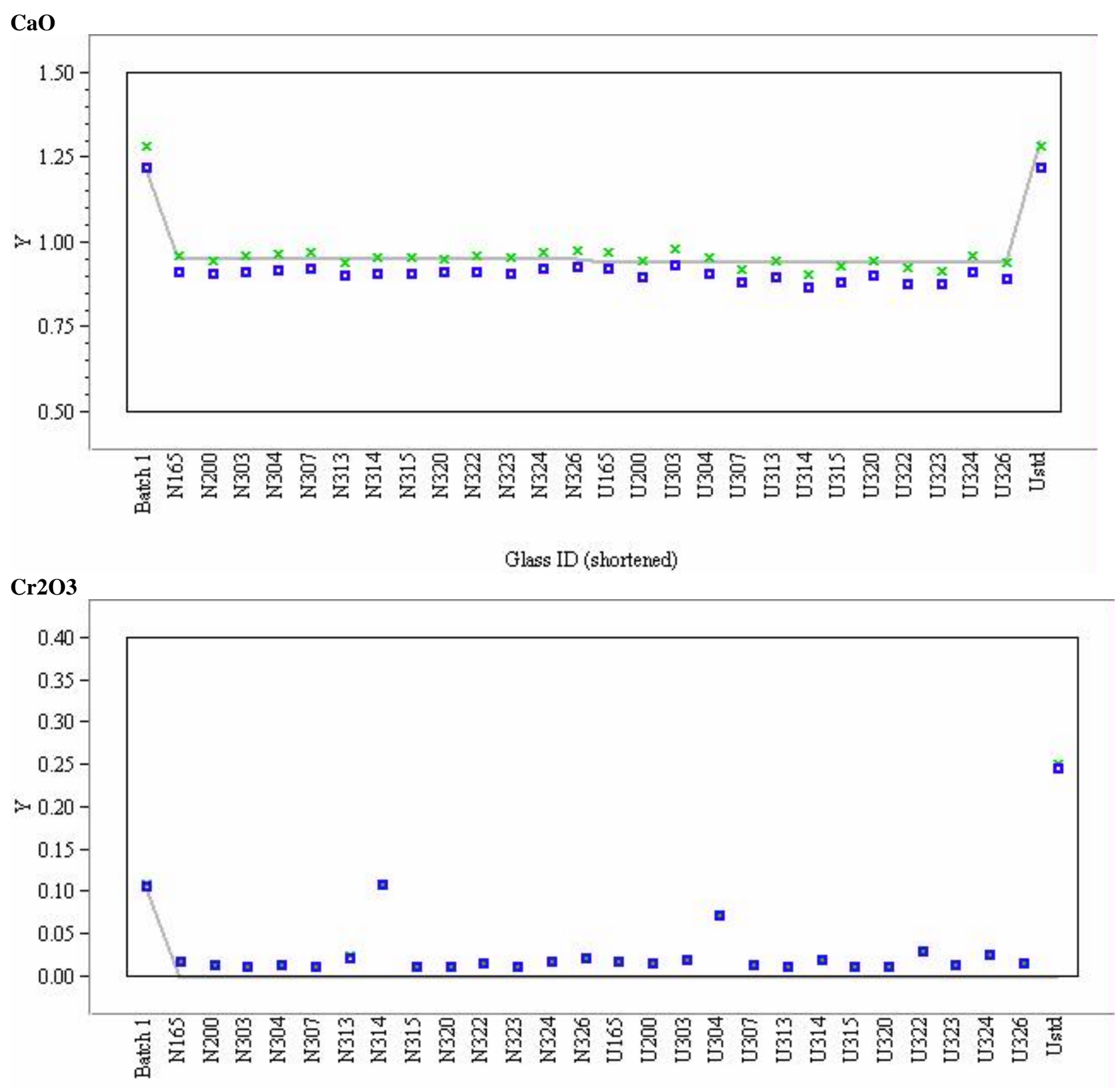

Glass ID (shortened) 
Immobilization Technology Section

WSRC-TR-2001-00131

Savannah River Technology Center

Rev. 0

Westinghouse Savannah River Company

Exhibit E7. Comparisons of Measured versus Targeted Chemical Compositions

(Concentrations in wt\%'s)
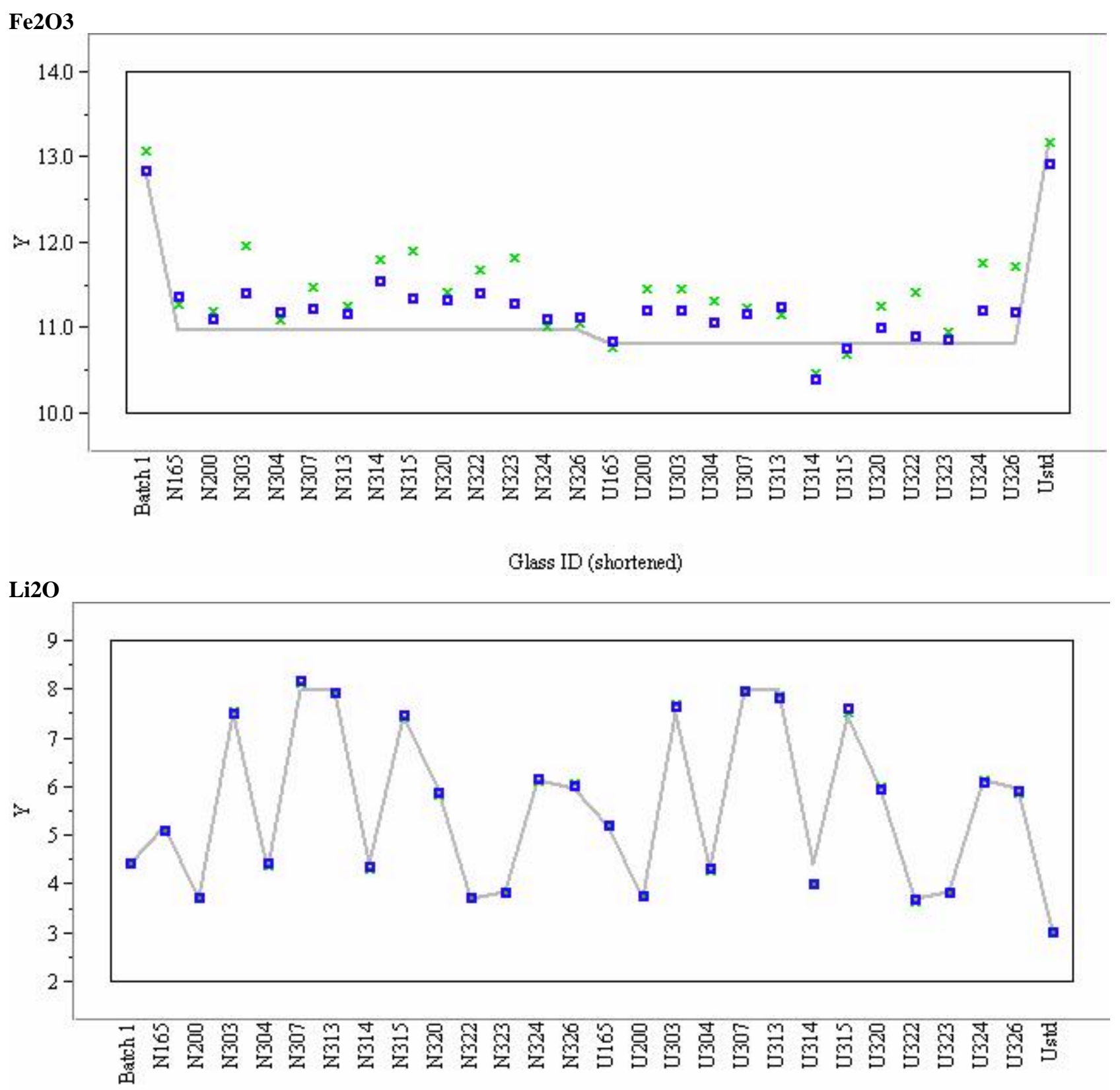

Glass ID (shortened)

$r$

-Tevered

* Moseral

- Mevaradbr 
Exhibit E7. Comparisons of Measured versus Targeted Chemical Compositions (Concentrations in wt\%'s)
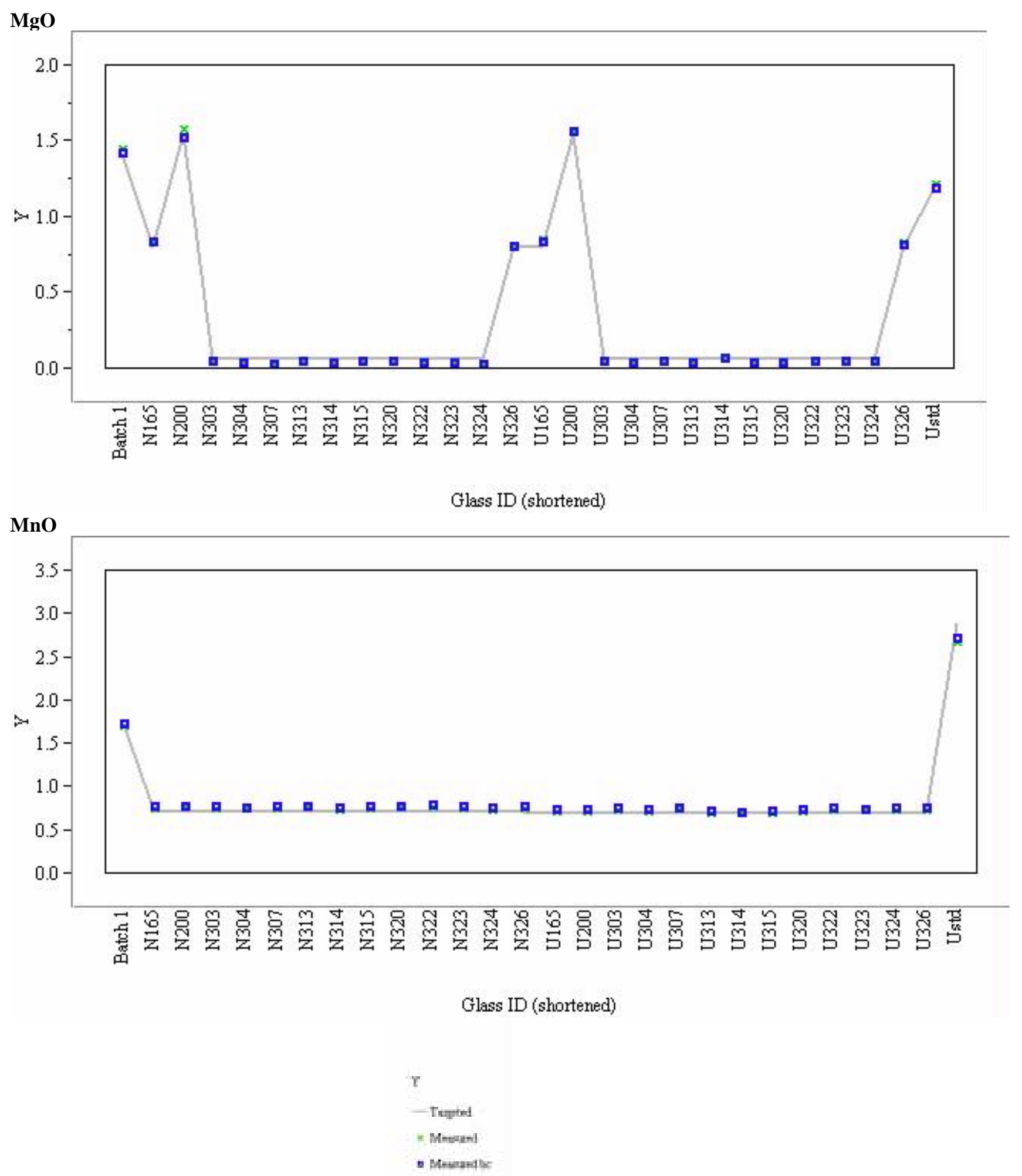
Exhibit E7. Comparisons of Measured versus Targeted Chemical Compositions

(Concentrations in wt\%'s)
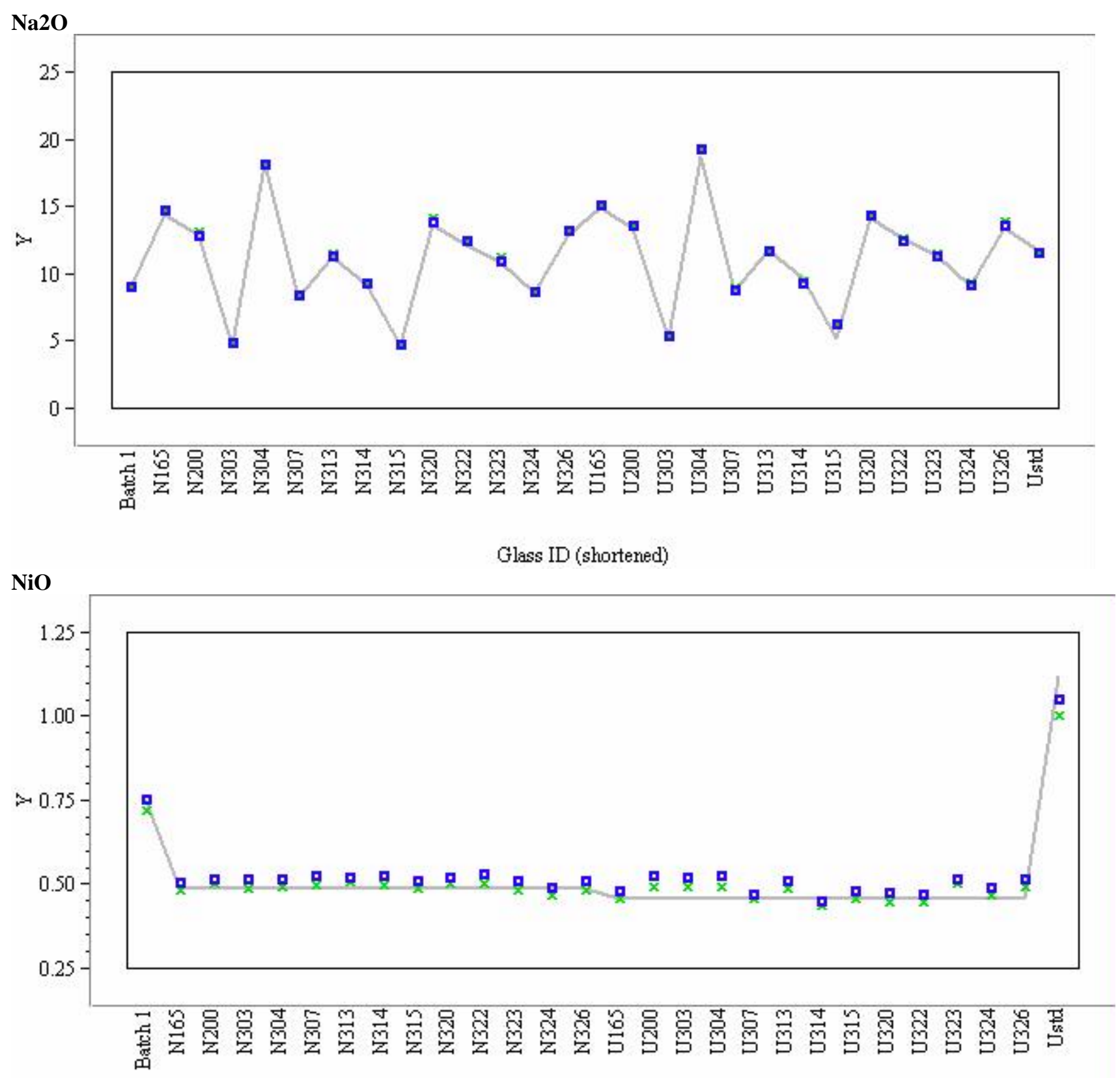

Glass ID (shortened)

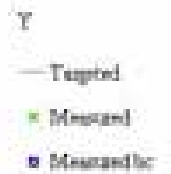


Exhibit E7. Comparisons of Measured versus Targeted Chemical Compositions

(Concentrations in wt\%'s)
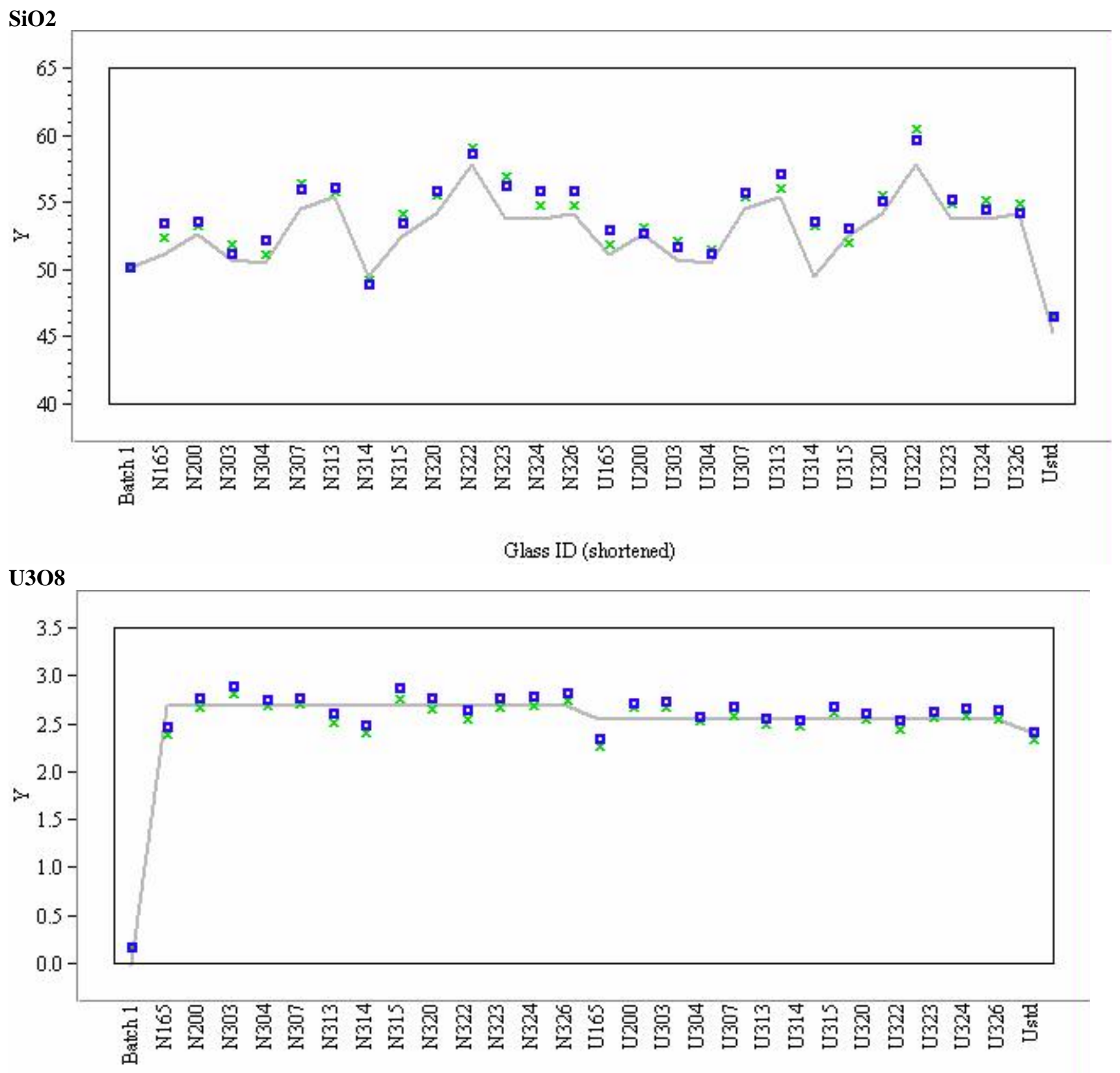

Glass ID (shortened)

$\checkmark$

-Tepost

* Musent

- Homanda 
Exhibit E7. Comparisons of Measured versus Targeted Chemical Compositions

$$
\text { (Concentrations in wt \%'s) }
$$

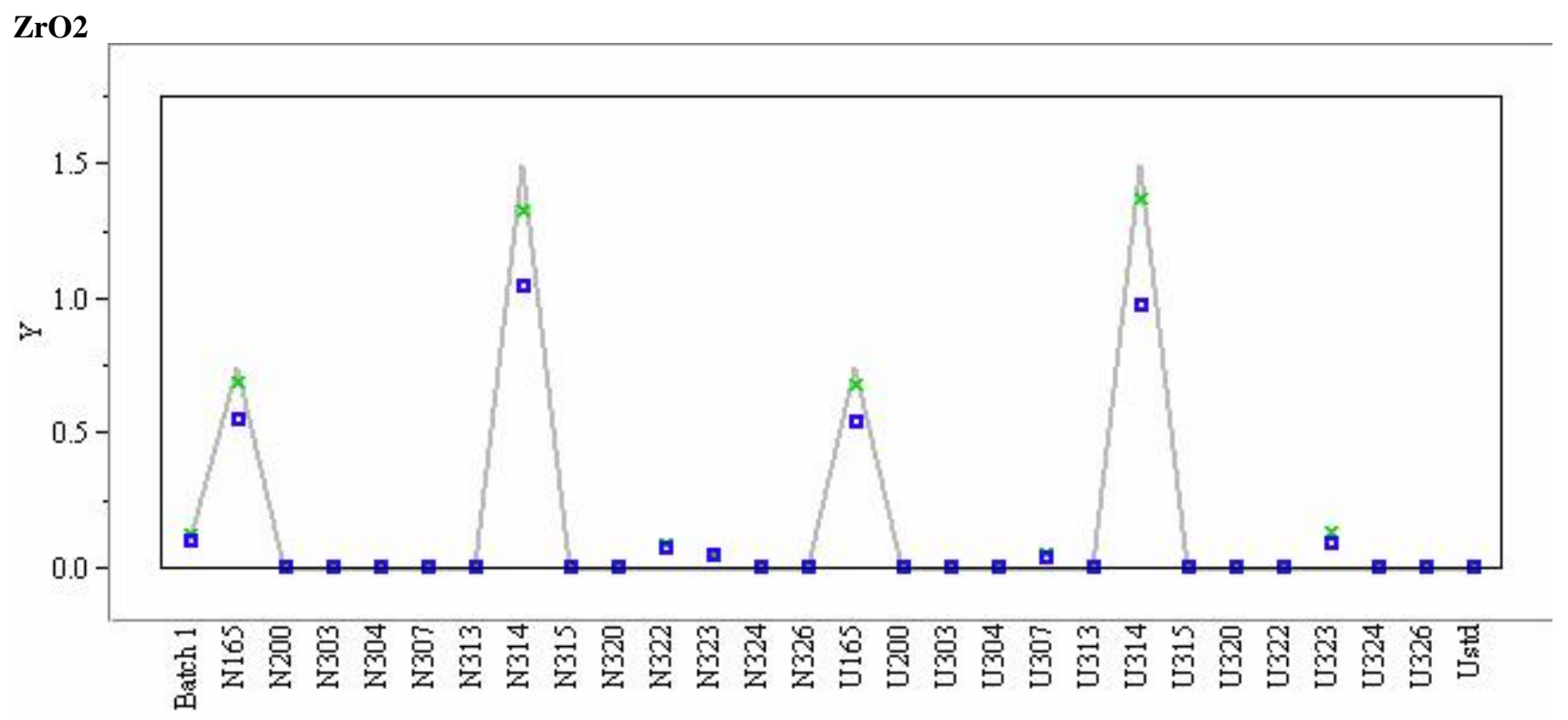

\section{Sum of Oxides}

Glass ID (shortened)

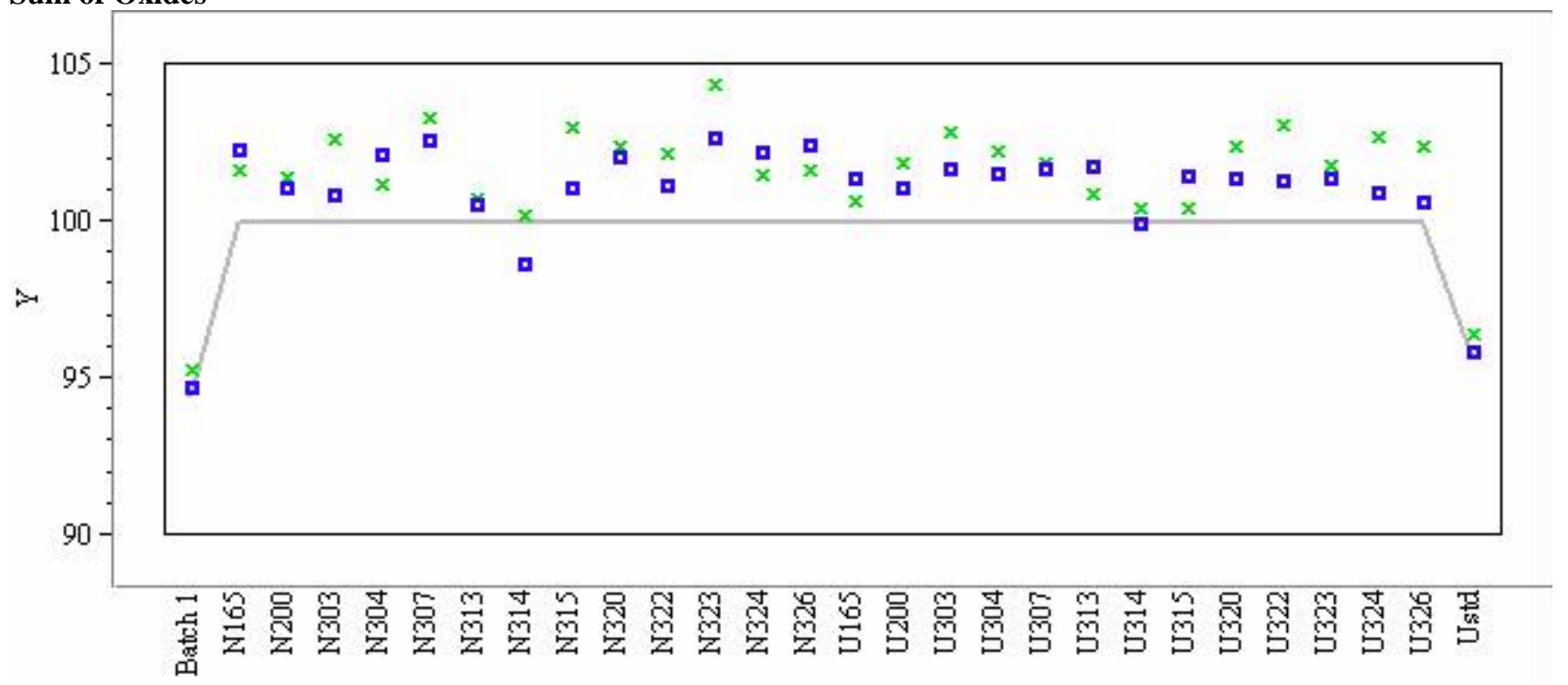

Glass ID (shortened)

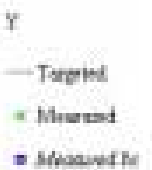


Exhibit E8. PCTs for Glasses from Nominally-Washed Sludge in Analytical Sequence (with results for EA and Blanks)

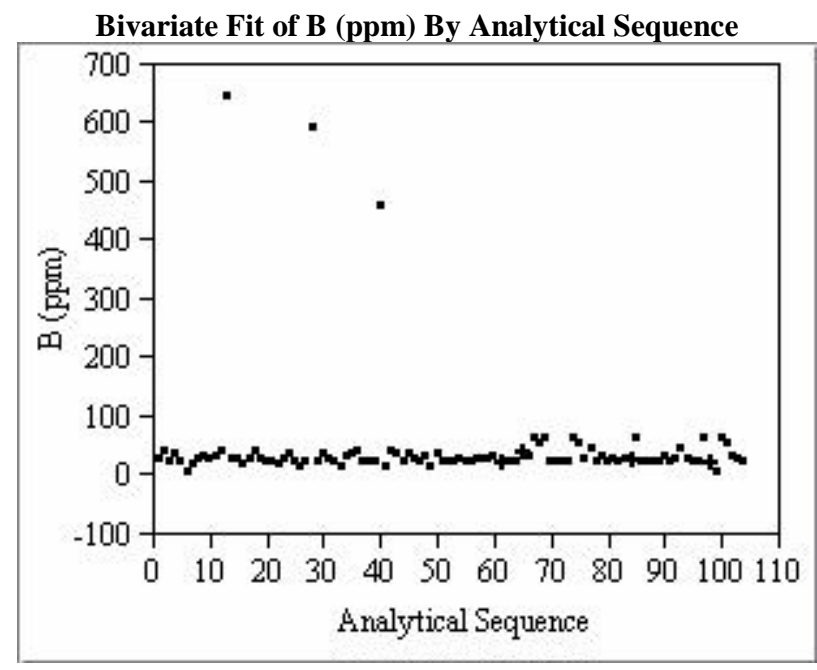

Bivariate Fit of Li (ppm) By Analytical Sequence

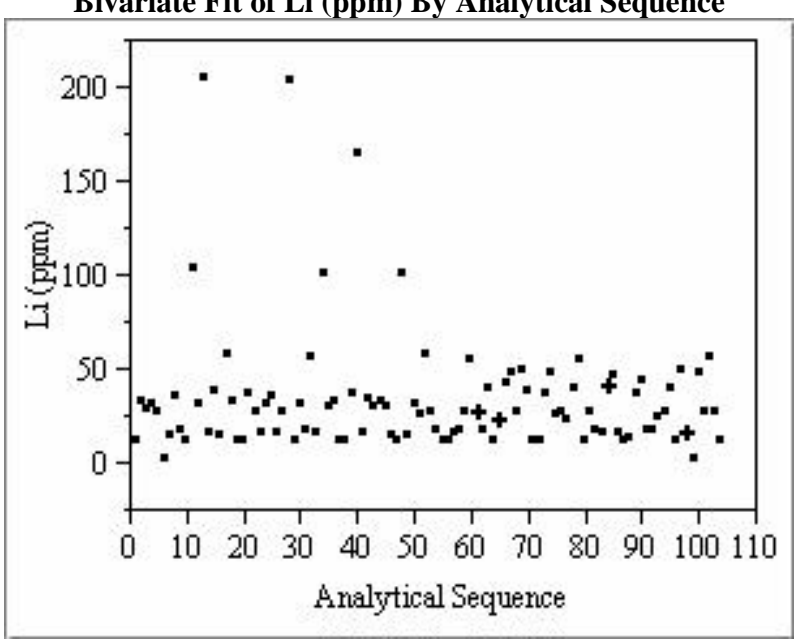

Bivariate Fit of Na (ppm) By Analytical Sequence

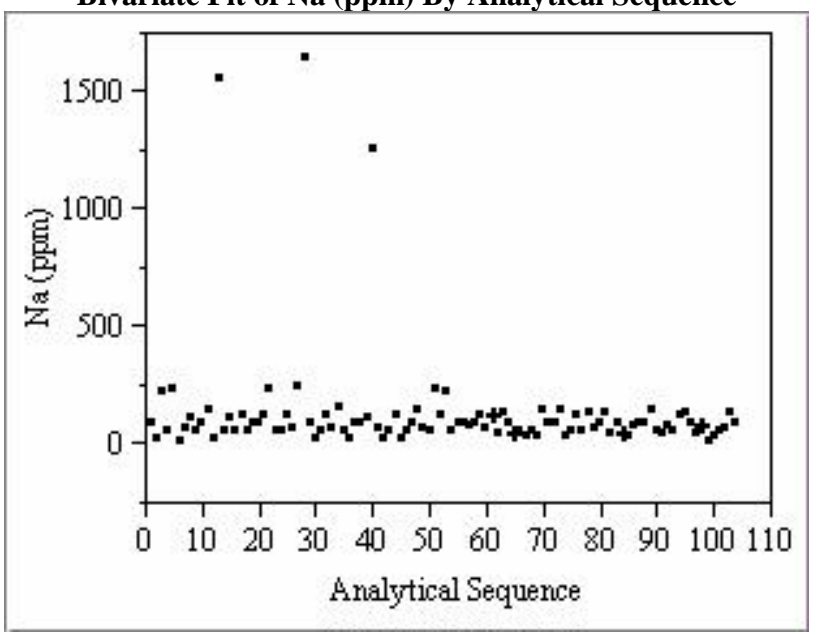

Bivariate Fit of Si (ppm) By Analytical Sequence

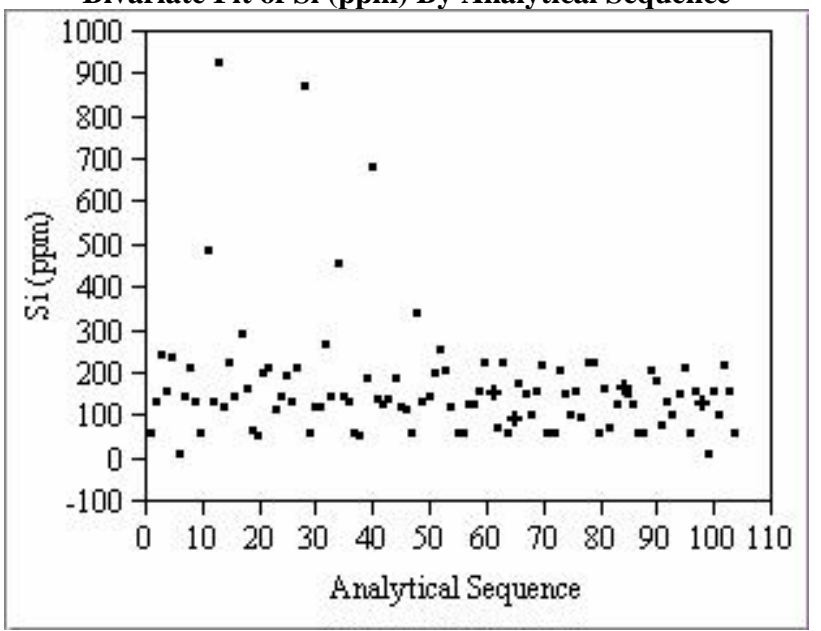


Exhibit E9. PCTs for Glasses from Nominally-Washed Sludge in Analytical Sequence (without results for EA \& Blanks)
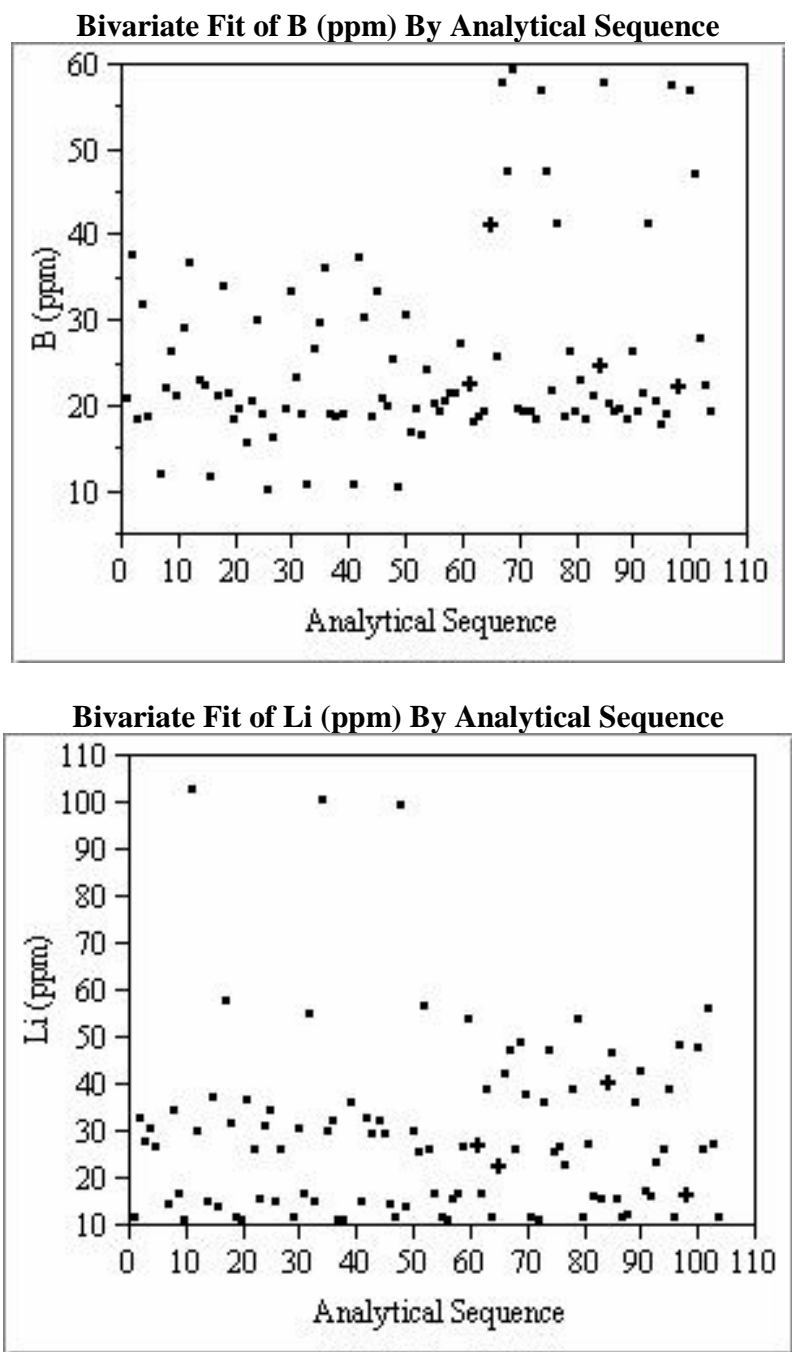

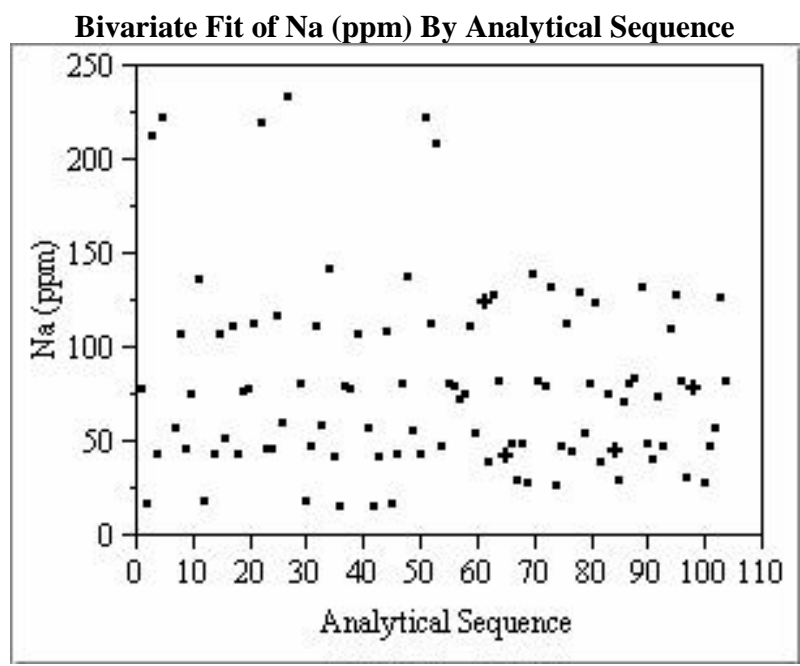

Bivariate Fit of Si (ppm) By Analytical Sequence

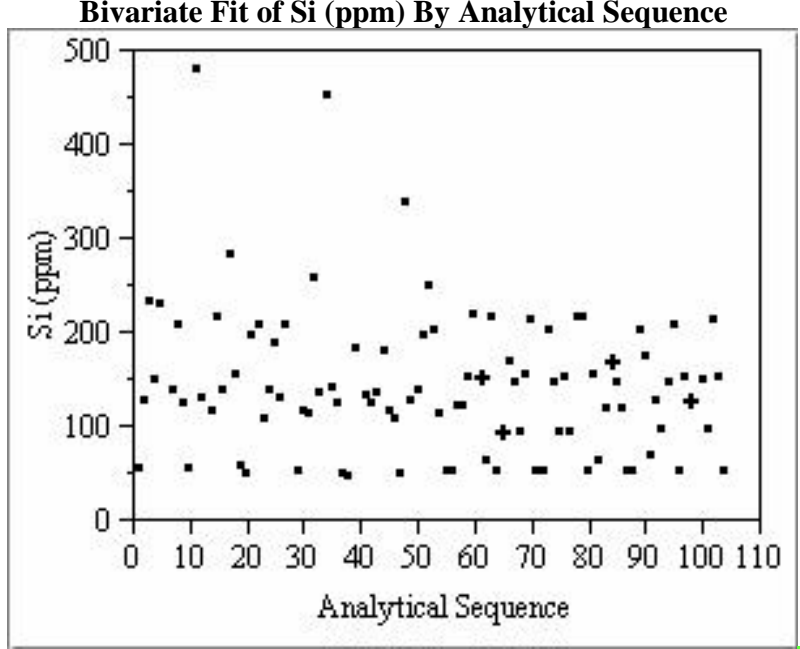


Exhibit E10. PCTs by Glass ID (Including EA and Blanks) for Nominally-Washed Sludge

Oneway Analysis of B (ppm) By Glass ID (shortened)

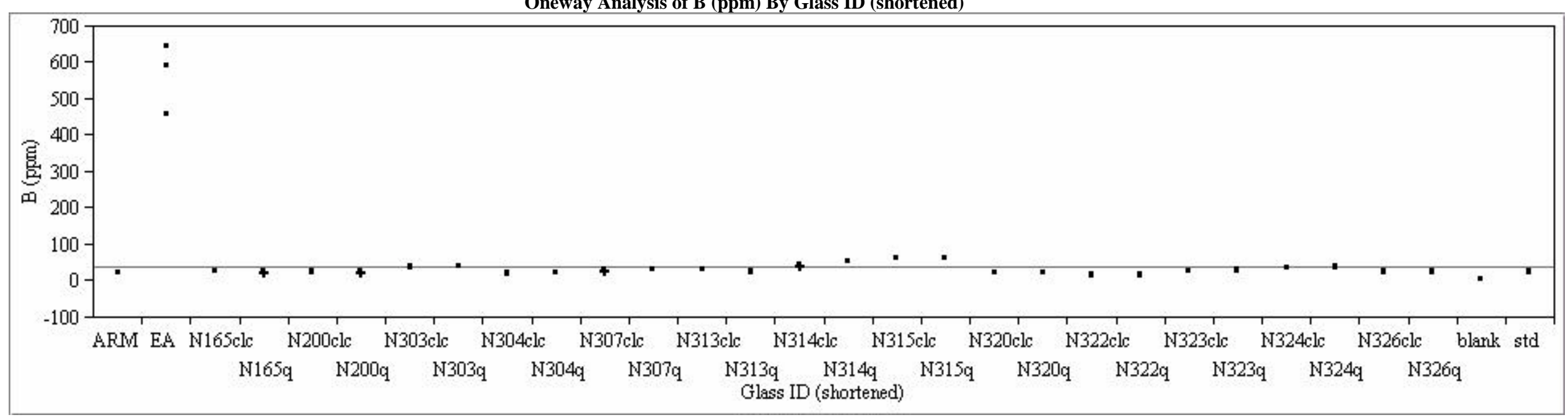

Oneway Analysis of Li (ppm) By Glass ID (shortened)

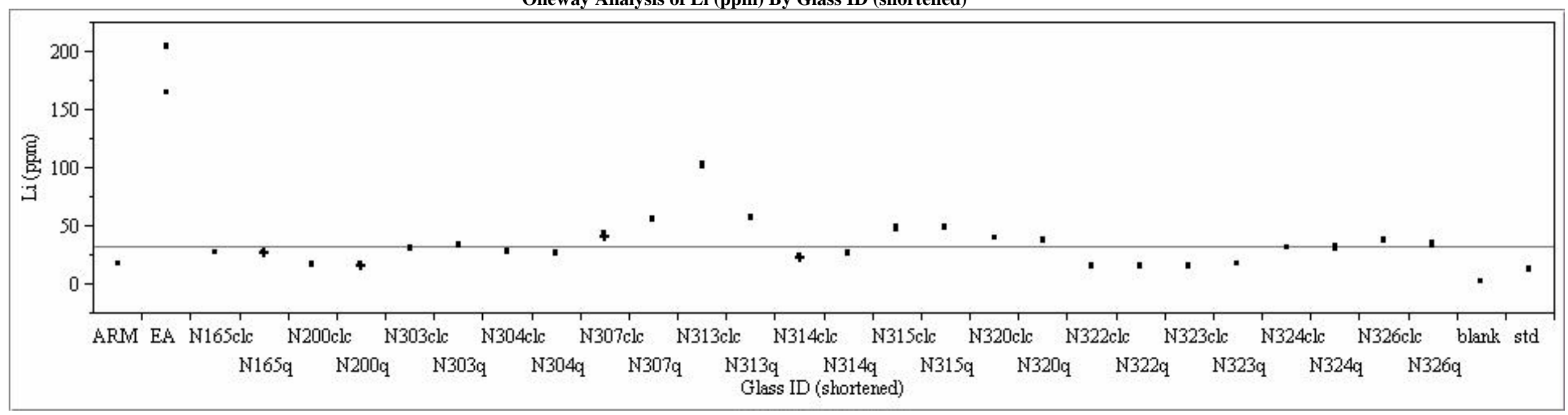


Exhibit E10. PCTs by Glass ID (Including EA and Blanks) for Nominally-Washed Sludge (continued)

Oneway Analysis of Na (ppm) By Glass ID (shortened)

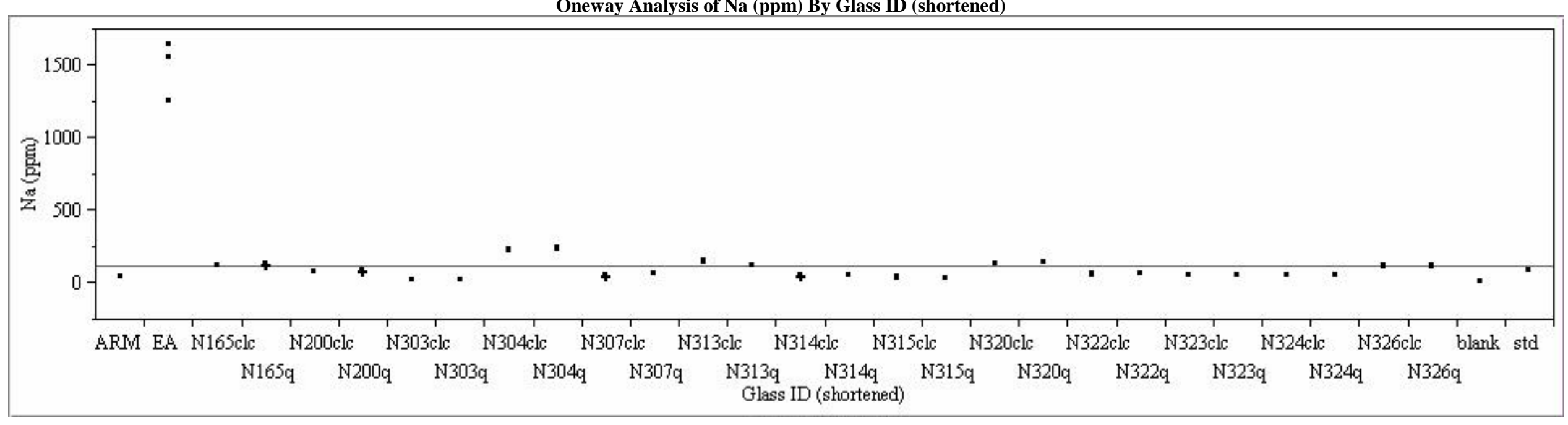

Oneway Analysis of Si (ppm) By Glass ID (shortened)

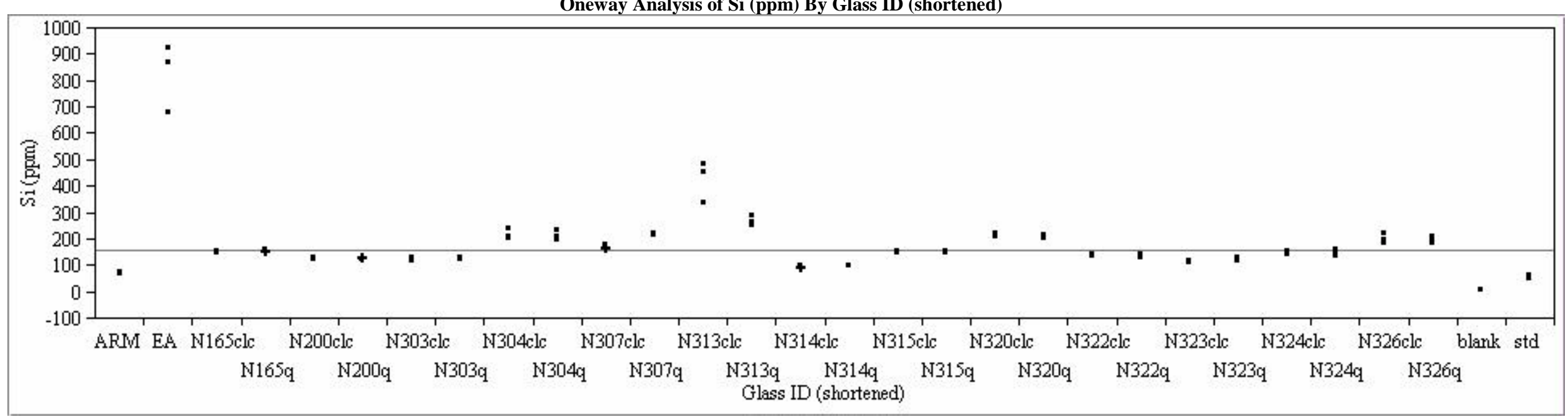


Exhibit E11. PCTs by Glass ID (Excluding EA and Blanks) for Nominally-Washed Sludge

Oneway Analysis of B (ppm) By Glass ID (shortened)

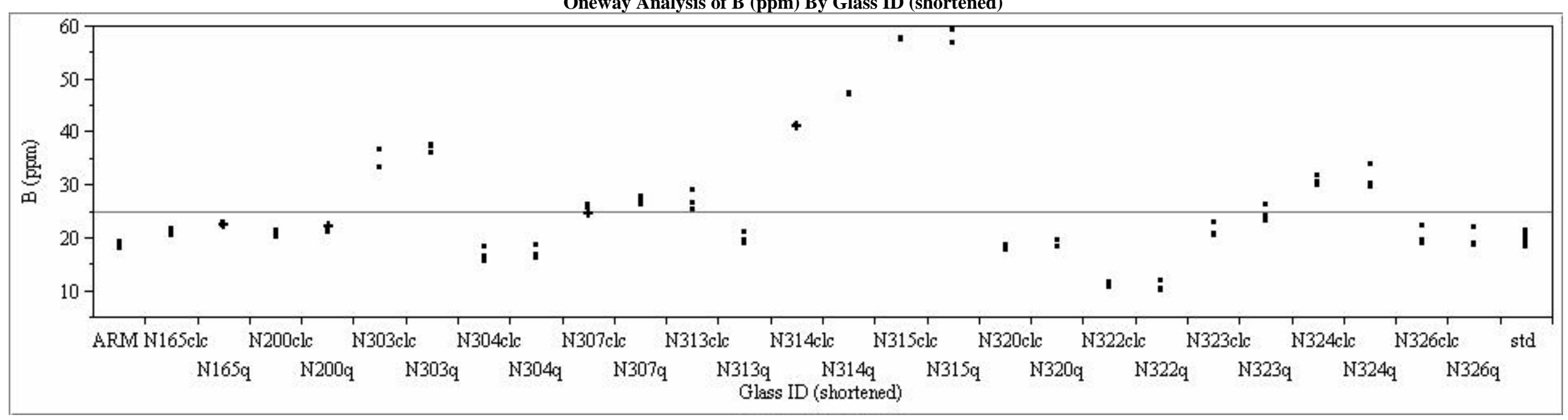

Oneway Analysis of Li (ppm) By Glass ID (shortened)

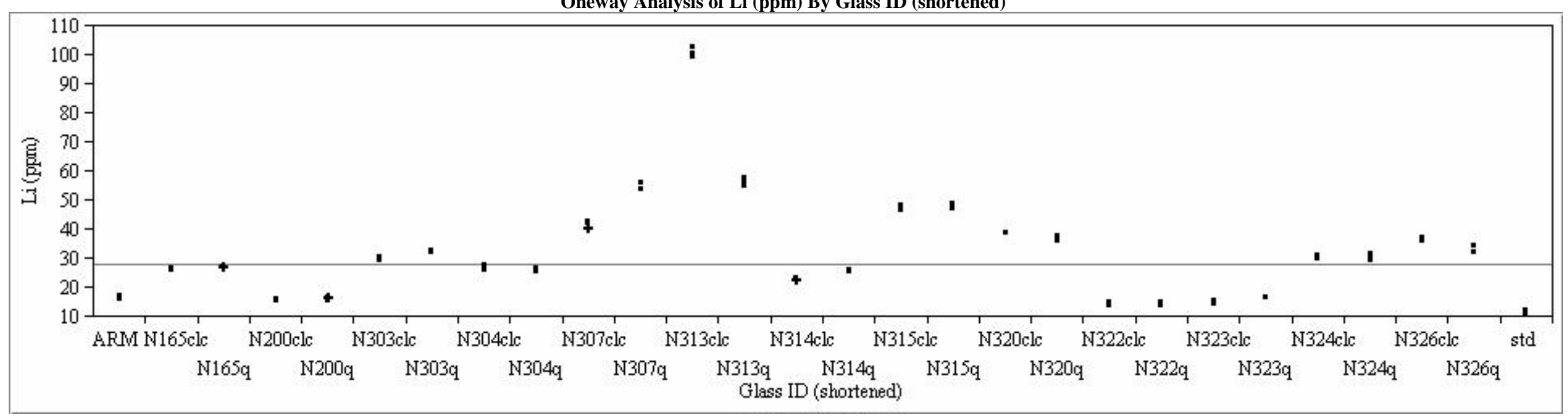


Exhibit E11. PCTs by Glass ID (Excluding EA and Blanks) for Nominally-Washed Sludge (continued)

Oneway Analysis of Na (ppm) By Glass ID (shortened)

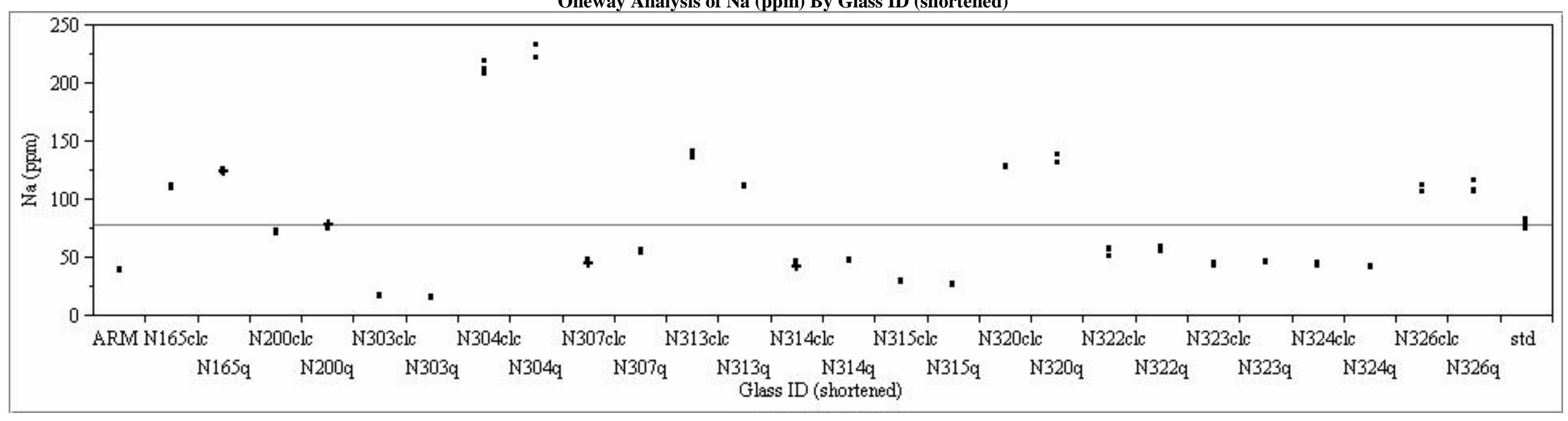

Oneway Analysis of Si (ppm) By Glass ID (shortened)

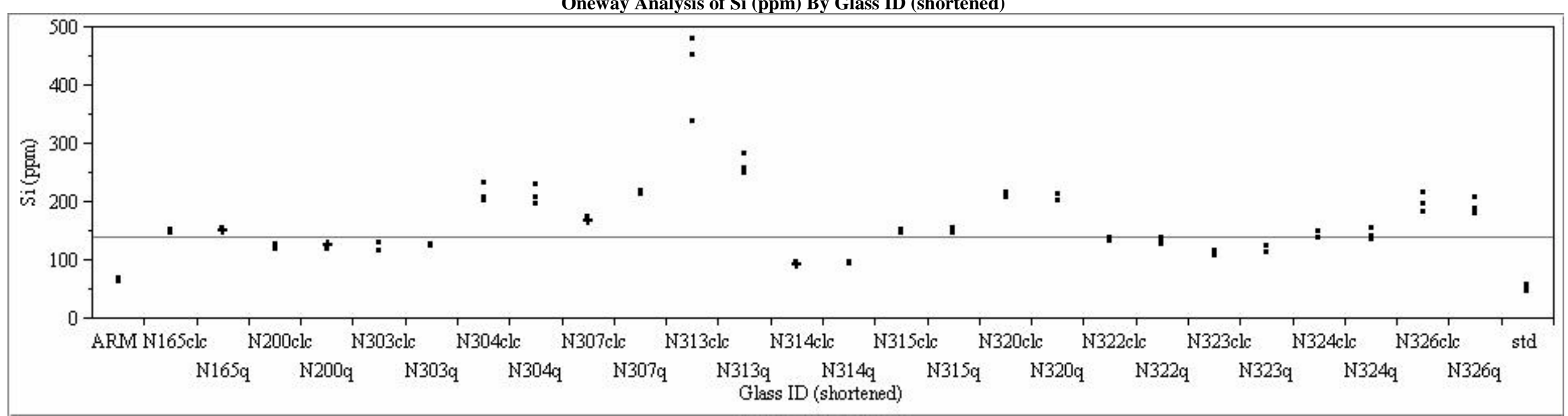




\section{Exhibit E12. Measurements of the Solutions Standards by ICP Block for Nominally-Washed Analyses}

Oneway Analysis of B (ppm) By Block

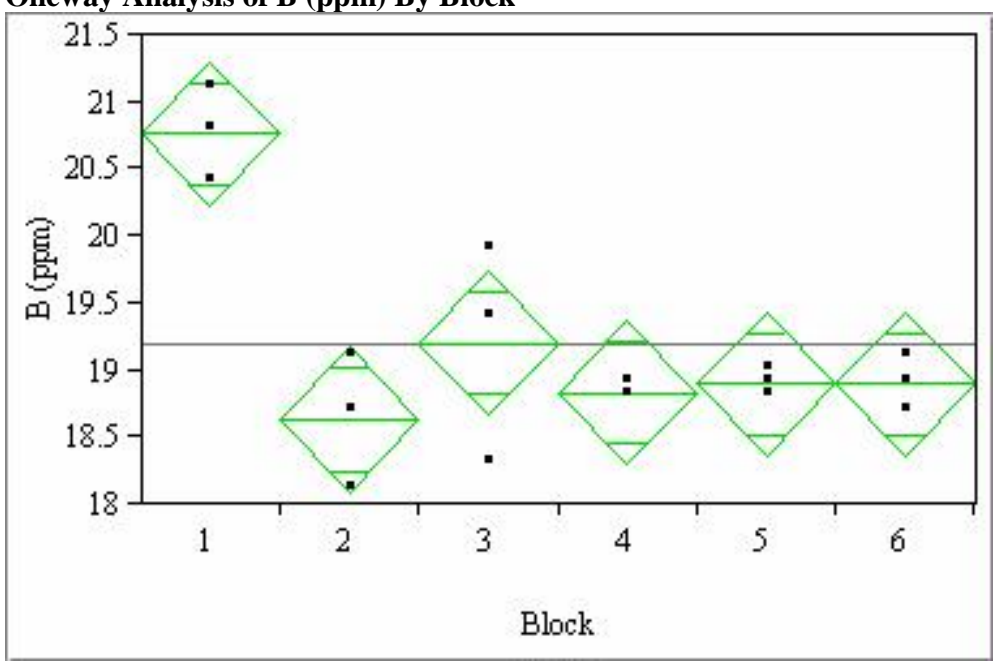

\section{Oneway Anova}

Summary of Fit

Rsquare

Adj Rsquare

Root Mean Square Erro

0.808186

0.728264

0.428174

Mean of Response

19.20556

Observations (or Sum Wgts)

18

Analysis of Variance

Source DF Sum of Squares Mean Square F Ratio Prob > F

$\begin{array}{lrrrrr}\text { Block } & 5 & 9.269444 & 1.85389 & 10.1121 & 0.0006\end{array}$

$\begin{array}{lrrr}\text { Block } & 5 & 9.269444 & 1.85389 \\ \text { Error } & 12 & 2.200000 & 0.18333\end{array}$

C. Total $17 \quad 11.469444$

Means for Oneway Anova

Level Number Mean Std Error Lower 95\% Upper 95\%

$\begin{array}{lllrrr}1 & 3 & 20.7667 & 0.24721 & 20.228 & 21.305\end{array}$

$\begin{array}{llllll}2 & 3 & 18.6333 & 0.24721 & 18.095 & 19.172\end{array}$

$\begin{array}{llllll}3 & 3 & 19.2000 & 0.24721 & 18.661 & 19.739 \\ 4 & 3 & 18.8333 & 0.24721 & 18.295 & 19.372\end{array}$

$\begin{array}{llllll}5 & 3 & 18.9000 & 0.24721 & 18.361 & 19.439\end{array}$

$\begin{array}{llllll}6 & 3 & 18.9000 & 0.24721 & 18.361 & 19.439\end{array}$

Std Error uses a pooled estimate of error variance
Oneway Analysis of Li (ppm) By Block

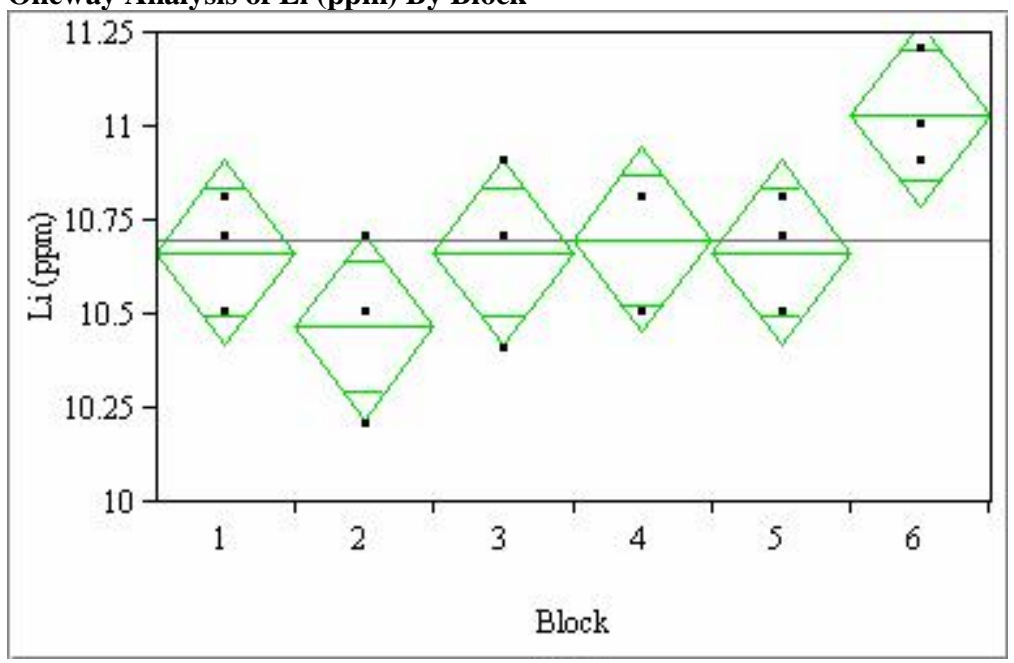

\section{Oneway Anova}

Summary of Fit

Rsquare

0.527778

Adj Rsquare

Root Mean Square Erro

0.194365

Mean of Response

10.7

Observations (or Sum Wgts)

Analysis of Variance

Source DF Sum of Squares Mean Square F Ratio Prob > F

$\begin{array}{lrrrrr}\text { Block } & 5 & 0.50666667 & 0.101333 & 2.6824 & 0.0749\end{array}$

$\begin{array}{lrrr}\text { Block } & 5 & 0.50666667 & 0.101333 \\ \text { Error } & 12 & 0.45333333 & 0.037778\end{array}$

C. Total $17 \quad 0.96000000$

Means for Oneway Anova

Level Number Mean Std Error Lower 95\% Upper 95\%

$\begin{array}{llllll}1 & 3 & 10.6667 & 0.11222 & 10.422 & 10.911\end{array}$

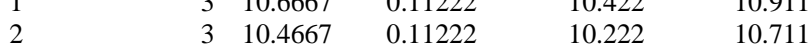

$\begin{array}{llllll}3 & 3 & 10.6667 & 0.11222 & 10.422 & 10.911\end{array}$

$\begin{array}{llllll}4 & 3 & 10.7000 & 0.11222 & 10.456 & 10.944 \\ 5 & 3 & 10.6667 & 0.1222 & 10.422 & 10.911\end{array}$

$\begin{array}{llllll}5 & 3 & 10.6667 & 0.11222 & 10.422 & 10.911 \\ 6 & 3 & 11.0333 & 0.11222 & 10.789 & 11.278\end{array}$

Std Error uses a pooled estimate of error variance 
Exhibit E12. Measurements of the Solutions Standards by ICP Block for Nominally-Washed Analyses (continued)

Oneway Analysis of $\mathrm{Na}(\mathrm{ppm})$ By Block

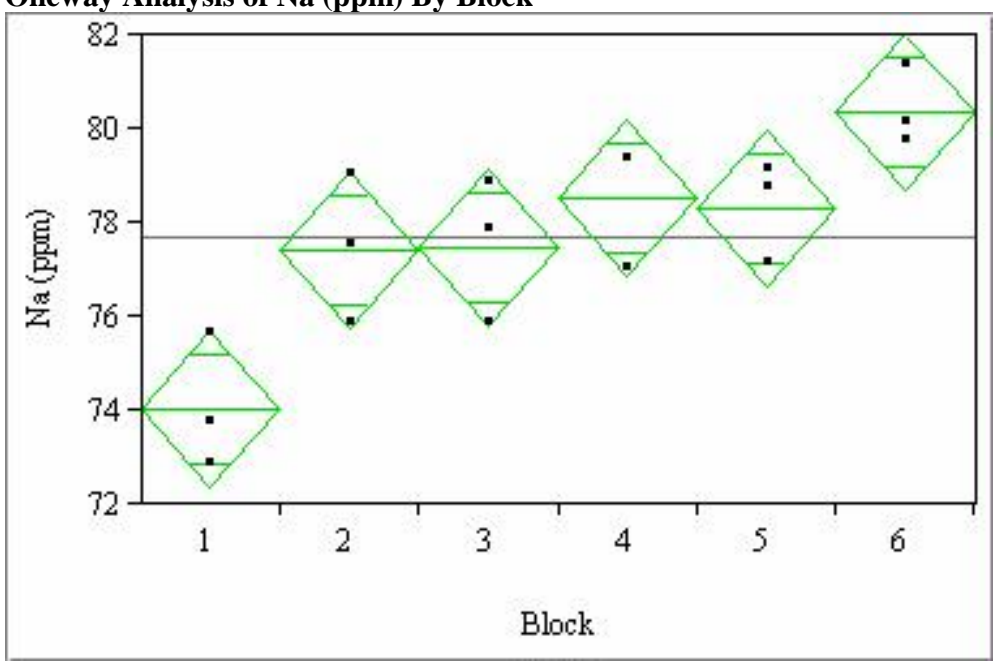

\section{Oneway Anova}

Summary of Fit

Rsquare

0.756101

Adj Rsquare

0.654476

Root Mean Sq

Mean of Response $\quad 77.68889$

Observations (or Sum Wgts)

Analysis of Variance

Source DF Sum of Squares Mean Square F Ratio Prob > F

$\begin{array}{lrrrrr}\text { Block } & 5 & 65.204444 & 13.0409 & 7.4401 & 0.0022\end{array}$

$\begin{array}{lrr}\text { Block } & 5 & 65.204444 \\ \text { Error } & 12 & 21.033333\end{array}$

$\begin{array}{lll}\text { C. Total } 17 & 17 & 86.237778\end{array}$

Means for Oneway Anova

Level Number Mean Std Error Lower 95\% Upper 95\%

$\begin{array}{llllll}1 & 3 & 74.0333 & 0.76437 & 72.368 & 75.699 \\ 2 & 3 & 77.4333 & 0.76437 & 75.768 & 79.099 \\ 3 & 3 & 77.4667 & 0.76437 & 75.801 & 79.132 \\ 4 & 3 & 78.5333 & 0.76437 & 76.868 & 80.199 \\ 5 & 3 & 78.3000 & 0.76437 & 76.635 & 79.965 \\ 6 & 3 & 80.3667 & 0.76437 & 78.701 & 82.032\end{array}$

Std Error uses a pooled estimate of error variance
Oneway Analysis of Si (ppm) By Block

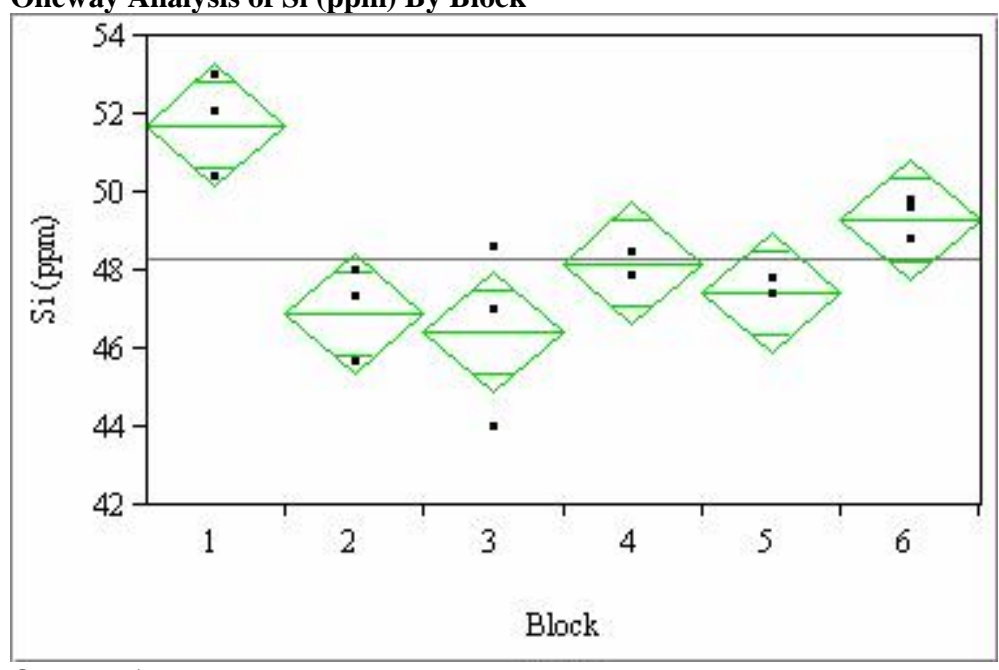

\section{Oneway Anova}

Summary of

Rsquare

0.759062

Adj Rsquare $\quad 0.658671$

Root Mean Square Error $\quad 1.227464$

Mean of Response

48.33333

Observations (or Sum Wgts)

Analysis of Variance

Source DF Sum of Squares Mean Square F Ratio Prob > F

$\begin{array}{lrrrrr}\text { Block } & 5 & 56.960000 & 11.3920 & 7.5611 & 0.0020\end{array}$

$\begin{array}{lrrr}\text { Block } & 5 & 56.960000 & 11.3920 \\ \text { Error } & 12 & 18.080000 & 1.5067\end{array}$

$\begin{array}{lll}\text { Error } & 12 & 18.080000 \\ \text { C. Total } & 17 & 75.040000\end{array}$

Means for Oneway Anova

Level Number Mean Std Error Lower 95\% Upper 95\%

$\begin{array}{llllll}1 & 3 & 51.7333 & 0.70868 & 50.189 & 53.277\end{array}$

$\begin{array}{llllll}1 & 3 & 46.9000 & 0.70868 & 45.356 & 48.444\end{array}$

$\begin{array}{llllll}3 & 3 & 46.4333 & 0.70868 & 44.889 & 47.977\end{array}$

$\begin{array}{llllll}4 & 3 & 48.2000 & 0.70868 & 46.656 & 49.744 \\ 5 & 3 & 47.4333 & 0.70868 & 45.889 & 48.977\end{array}$

$\begin{array}{llllll}5 & 3 & 47.4333 & 0.70868 & 45.889 & 48.977\end{array}$

$\begin{array}{lllll}3 & 49.3000 & 0.70868 & 47.756 & 50.844\end{array}$ 


\section{Exhibit E13. Correlations of PCTs for Nominally-Washed Case}

\section{Nominal-Measured Composition-clc}

\section{Correlations}

$\begin{array}{lrrrr} & \log \mathbf{N L}[\mathbf{B}(\mathrm{g} / \mathbf{L})] & \log \mathbf{N L}[\mathbf{L i}(\mathbf{g} / \mathbf{L})] & \log \mathbf{N L}[\mathbf{N a}(\mathbf{g} / \mathbf{L})] & \log \mathbf{N L}[\mathbf{S i}(\mathbf{g} / \mathbf{L})] \\ \log \mathbf{N L}[\mathbf{B}(\mathbf{g} / \mathbf{L})] & 1.0000 & 0.9478 & 0.7372 & 0.8286 \\ \log \mathbf{N L}[\mathbf{L i}(\mathbf{g} / \mathbf{L})] & 0.9478 & 1.0000 & 0.7495 & 0.9039 \\ \log \mathbf{N L}[\mathbf{N a}(\mathbf{g} / \mathbf{L})] & 0.7372 & 0.7495 & 1.0000 & 0.8453 \\ \log \mathbf{N L}[\mathbf{S i}(\mathbf{g} / \mathbf{L})] & 0.8286 & 0.9039 & 0.8453 & 1.0000\end{array}$

\section{Scattenplot Matrix}

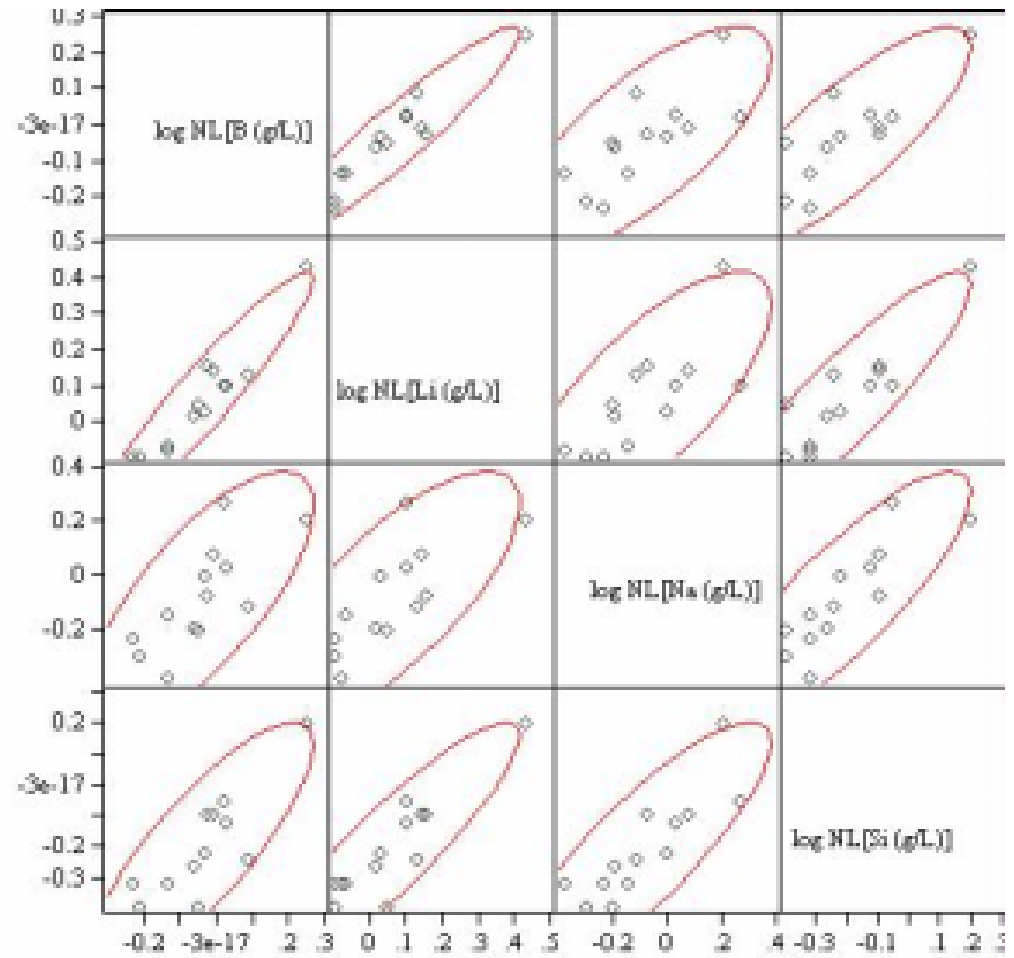

\section{Nominal-Measured Composition-quenched}

\section{Correlations}

$\begin{array}{rrrr}\log \mathrm{NL}[\mathrm{B}(\mathrm{g} / \mathrm{L})] & \log \mathrm{NL}[\mathrm{Li}(\mathrm{g} / \mathrm{L})] & \log \mathrm{NL}[\mathrm{Na}(\mathrm{g} / \mathrm{L})] \quad \log \mathrm{NL}[\mathrm{Si}(\mathrm{g} / \mathrm{L})]\end{array}$

$\begin{array}{lllll}\log \mathbf{N L}[\mathbf{B}(\mathbf{g} / \mathbf{L})] & 1.0000 & 0.9620 & 0.5915 & 0.6687 \\ \log \mathbf{N}[\mathbf{L i}(\mathbf{g} / \mathbf{L})] & 0.9620 & 1.0000 & 0.6407 & 0.6932 \\ \log \mathbf{N}[\mathbf{N a}(\mathbf{g} / \mathbf{L})] & 0.5915 & 0.6407 & 1.0000 & 0.8124 \\ \log \mathbf{N}[\mathbf{S i}(\mathbf{g} / \mathbf{L})] & 0.6687 & 0.6932 & 0.8124 & 1.0000\end{array}$

\section{Scattenplot Matnix}

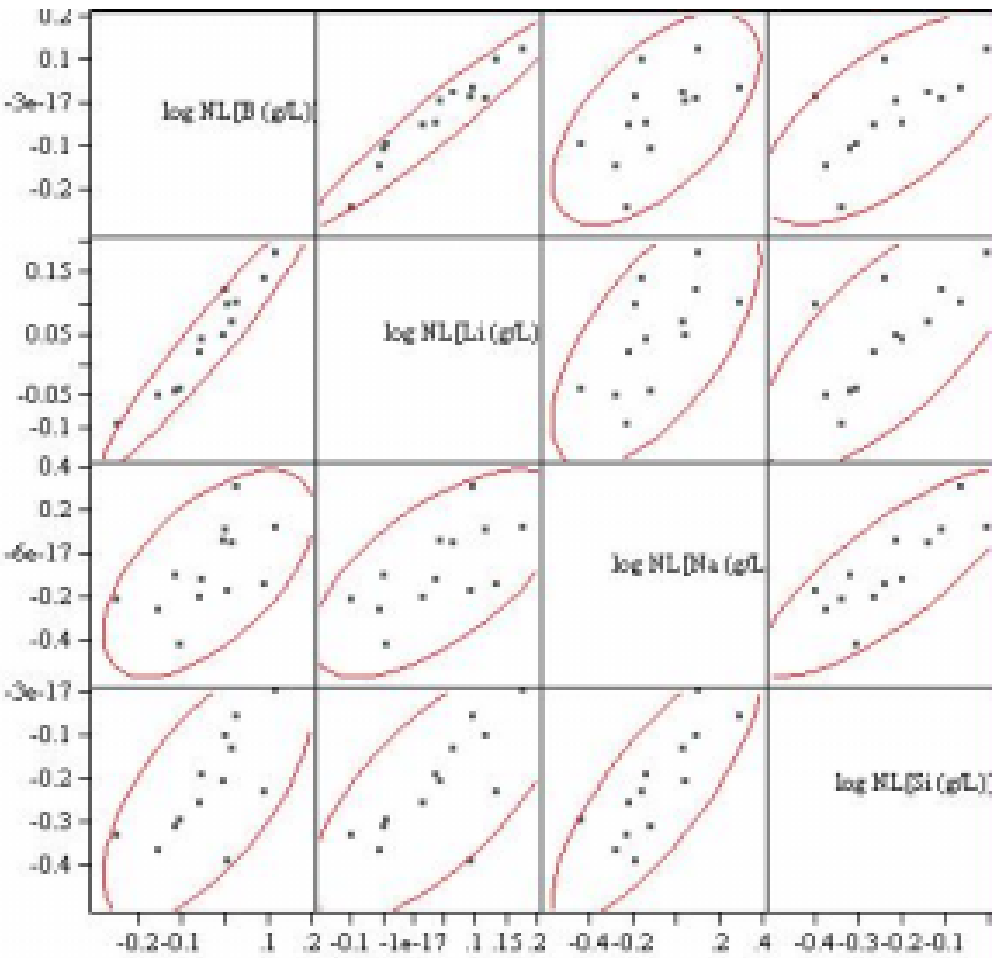


Exhibit E13. Correlations of PCTs for Nominally-Washed Case (Continued)

\section{Nominal-Measured bc-clc}

\section{Correlations}

$\begin{array}{lrrrr} & \log \mathbf{N L}[\mathbf{B}(\mathbf{g} / \mathbf{L})] & \log \mathbf{N L}[\mathbf{L i}(\mathbf{g} / \mathbf{L})] & \log \mathbf{N L}[\mathbf{N a}(\mathbf{g} / \mathbf{L})] & \log \mathbf{N L}[\mathbf{S i}(\mathbf{g} / \mathbf{L})] \\ \log \mathbf{N L}[\mathbf{B}(\mathbf{g} / \mathbf{L})] & 1.0000 & 0.9456 & 0.7282 & 0.8151 \\ \log \mathbf{N L} \mathbf{L i}(\mathbf{g} / \mathbf{L})] & 0.9456 & 1.0000 & 0.7519 & 0.9101 \\ \log \mathbf{N L}[\mathbf{N a}(\mathbf{g} / \mathbf{L})] & 0.7282 & 0.7519 & 1.0000 & 0.8380 \\ \log \mathbf{N L}[\mathbf{S i}(\mathbf{g} / \mathbf{L})] & 0.8151 & 0.9101 & 0.8380 & 1.0000\end{array}$

\section{Scattenplot Matrix}

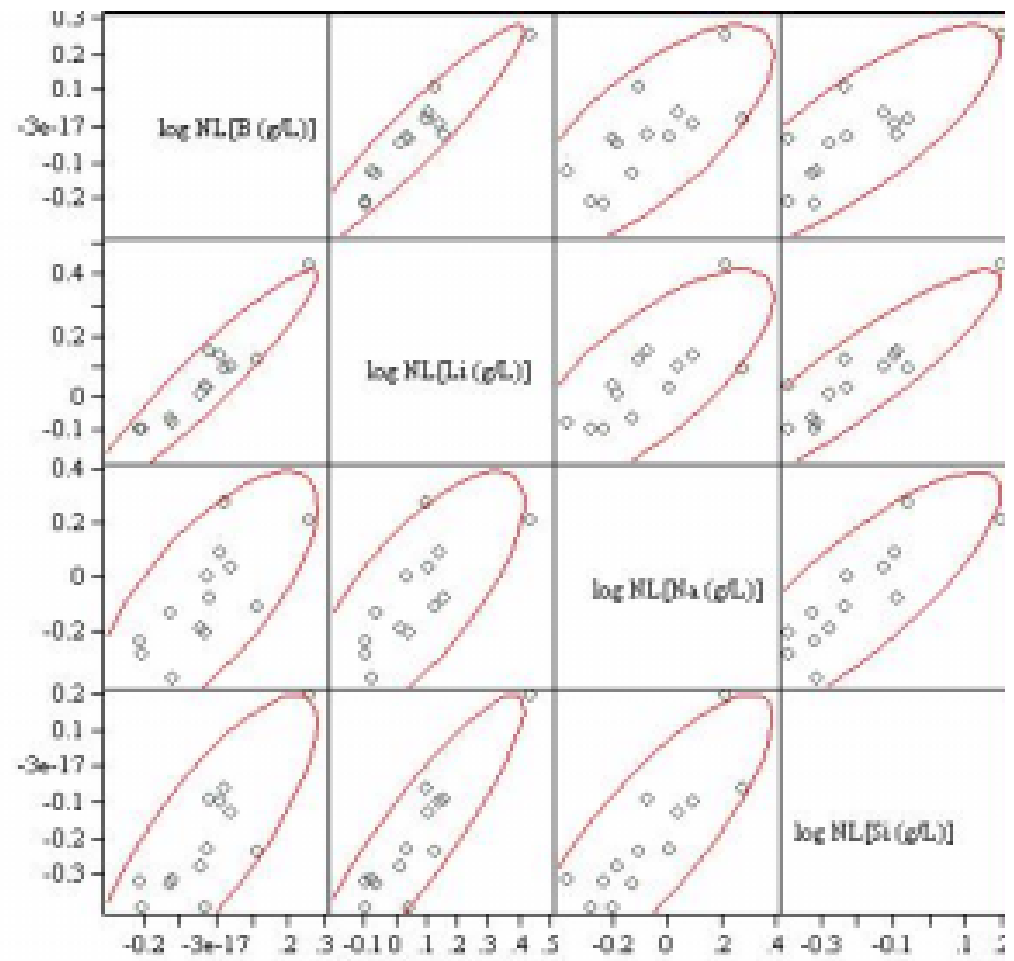

Nominal-Measured bc-quenched

\section{Correlations}

$\begin{array}{rrrr}\log \mathrm{NL}[\mathrm{B}(\mathrm{g} / \mathrm{L})] & \log \mathrm{NL}[\mathrm{Li}(\mathrm{g} / \mathrm{L})] & \log \mathrm{NL}[\mathrm{Na}(\mathrm{g} / \mathrm{L})] \quad \log \mathrm{NL}[\mathrm{Si}(\mathrm{g} / \mathrm{L})]\end{array}$

$\begin{array}{lrrrr}\log \mathbf{N L}[\mathbf{B}(\mathbf{g} / \mathbf{L})] & 1.0000 & 0.9717 & 0.5767 & 0.6501 \\ \log \mathbf{N L}[\mathbf{L i}(\mathbf{g} / \mathbf{L})] & 0.9717 & 1.0000 & 0.6443 & 0.7040 \\ \log \mathbf{N L}[\mathbf{N a}(\mathbf{g} / \mathbf{L})] & 0.5767 & 0.6443 & 1.0000 & 0.8040 \\ \log \mathbf{N L}[\mathbf{S i}(\mathbf{g} / \mathbf{L})] & 0.6501 & 0.7040 & 0.8040 & 1.0000\end{array}$

\section{Scattenplot Matnix}

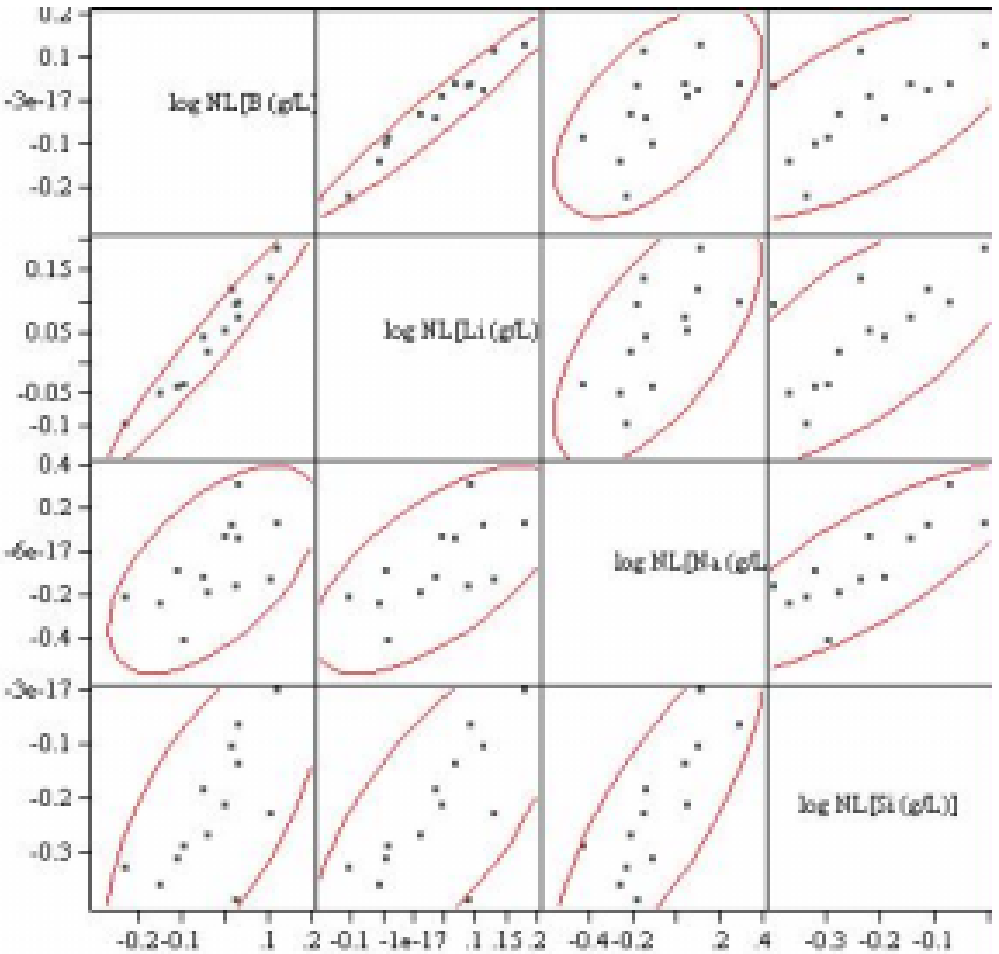


Exhibit E13. Correlations of PCTs for Nominally-Washed Case (Continued)

\section{Nominal-Targeted-clc \\ Correlations}

$\begin{array}{lrrrr} & \log \mathbf{N L}[\mathbf{B}(\mathbf{g} / \mathbf{L})] & \log \mathbf{N L}[\mathbf{L i}(\mathbf{g} / \mathbf{L})] & \log \mathbf{N L}[\mathbf{N a}(\mathbf{g} / \mathbf{L})] & \log \mathbf{N L}[\mathbf{S i}(\mathbf{g} / \mathbf{L})] \\ \log \mathbf{N L}[\mathbf{B}(\mathbf{g} / \mathbf{L})] & 1.0000 & 0.9444 & 0.7708 & 0.8194 \\ \log \mathbf{N L}[\mathbf{L i}(\mathbf{g} / \mathbf{L})] & 0.9444 & 1.0000 & 0.7941 & 0.9082 \\ \log \mathbf{N L}[\mathbf{N a}(\mathbf{g} / \mathbf{L})] & 0.7708 & 0.7941 & 1.0000 & 0.8678 \\ \log \mathbf{N L}[\mathbf{S i}(\mathbf{g} / \mathbf{L})] & 0.8194 & 0.9082 & 0.8678 & 1.0000\end{array}$

\section{Scattenplot Matrix}

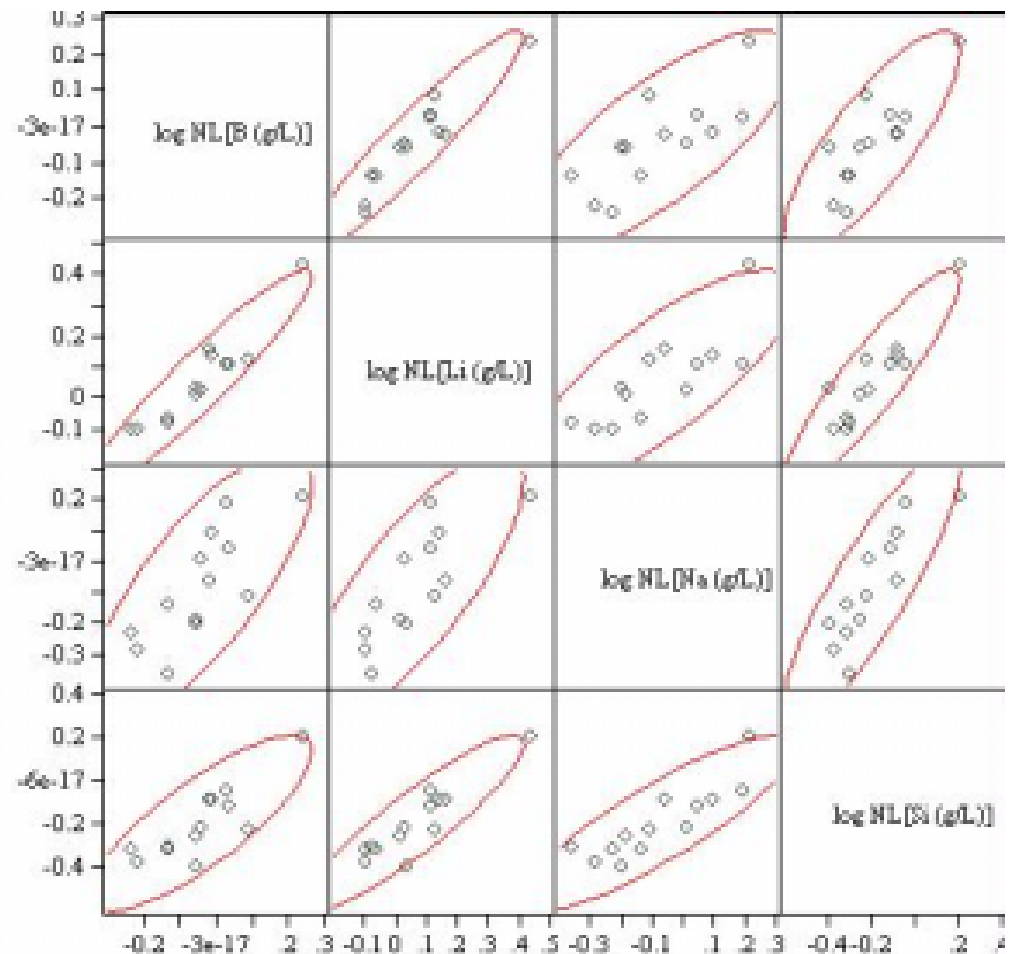

\section{Nominal-Targeted-quenched}

Correlations

$\begin{array}{lrrrr} & \log \mathbf{N L}[\mathbf{B}(\mathbf{g} / \mathbf{L})] & \log \mathbf{N L}[\mathbf{L i}(\mathbf{g} / \mathbf{L})] & \log \mathbf{N L}[\mathbf{N a}(\mathbf{g} / \mathbf{L})] & \log \mathbf{N L}[\mathbf{S i}(\mathbf{g} / \mathbf{L})] \\ \log \mathbf{N L}[\mathbf{B}(\mathbf{g} / \mathbf{L})] & 1.0000 & 0.9639 & 0.6058 & 0.6549 \\ \log \mathbf{N L} \mathbf{L i}(\mathbf{g} / \mathbf{L})] & 0.9639 & 1.0000 & 0.6712 & 0.7058 \\ \log \mathbf{N L}[\mathbf{N a}(\mathbf{g} / \mathbf{L})] & 0.6058 & 0.6712 & 1.0000 & 0.8185 \\ \log \mathbf{N L}[\mathbf{S i}(\mathbf{g} / \mathbf{L})] & 0.6549 & 0.7058 & 0.8185 & 1.0000\end{array}$

\section{Scattenplot Matrix}

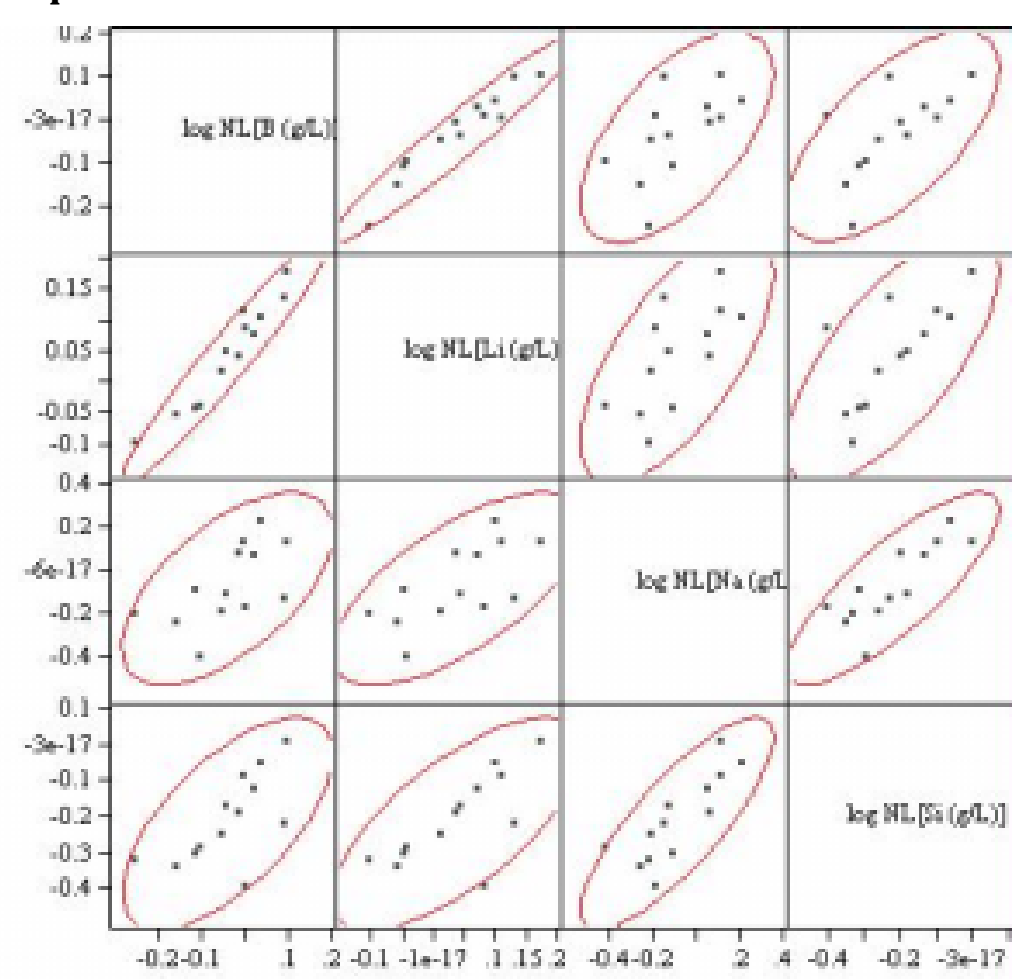


Exhibit E14. PCTs for Glasses from Underwashed Sludge in Analytical Sequence (with results for EA and Blanks)

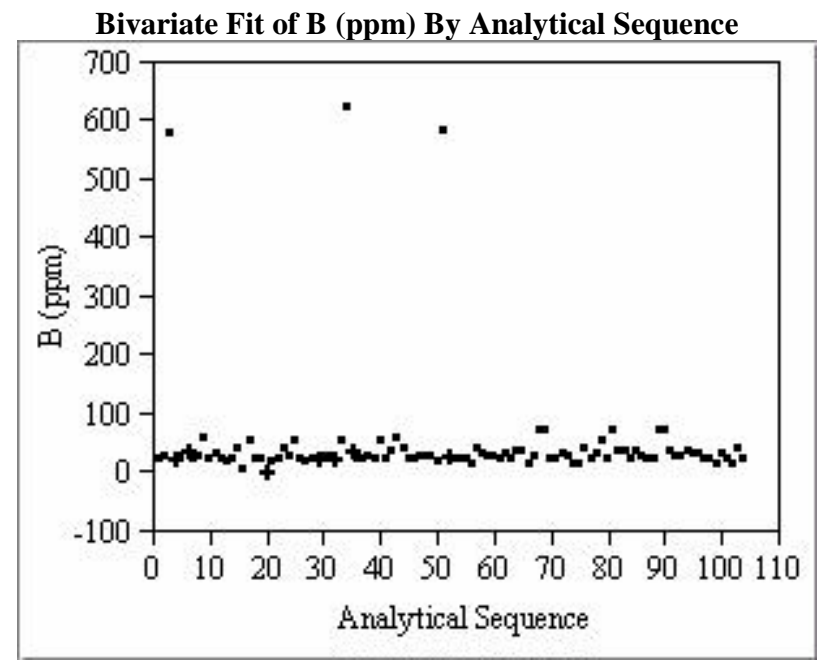

Bivariate Fit of Li (ppm) By Analytical Sequence

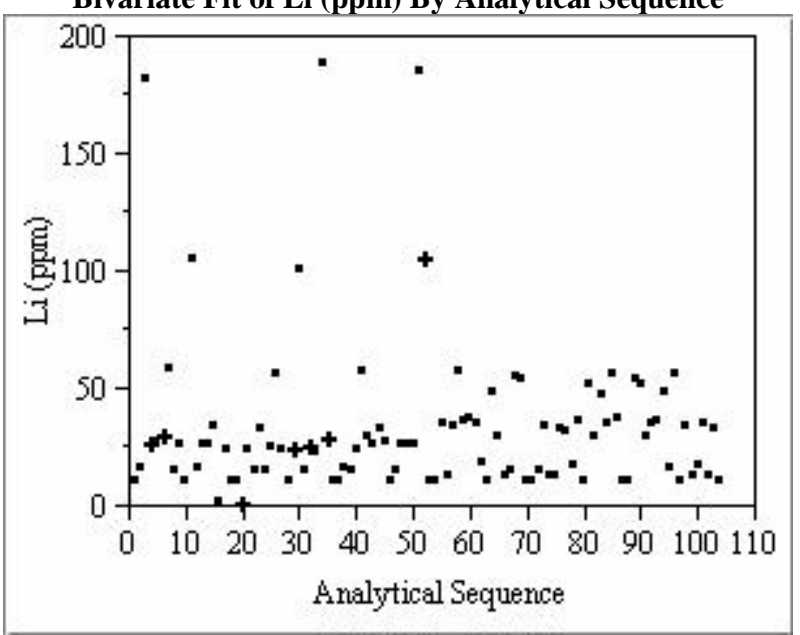

Bivariate Fit of Na (ppm) By Analytical Sequence

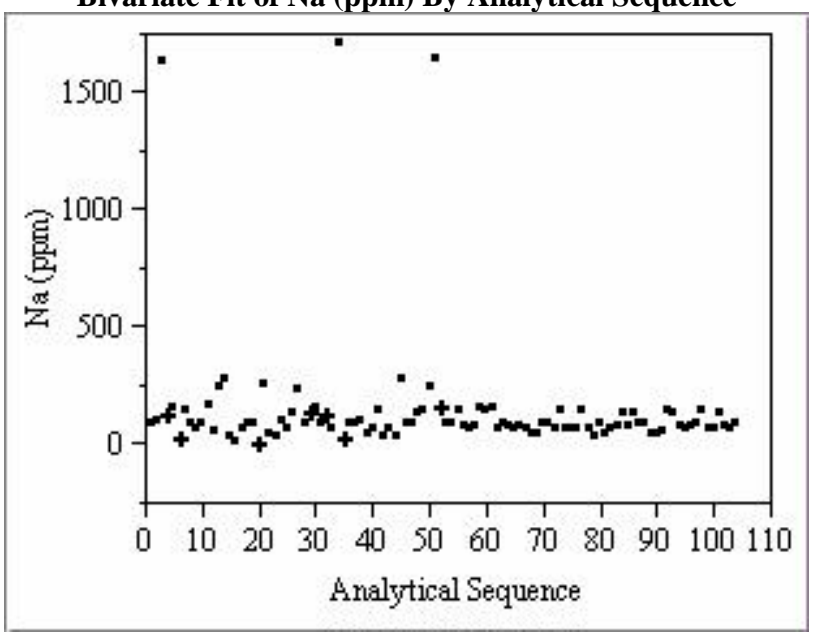

Bivariate Fit of Si (ppm) By Analytical Sequence

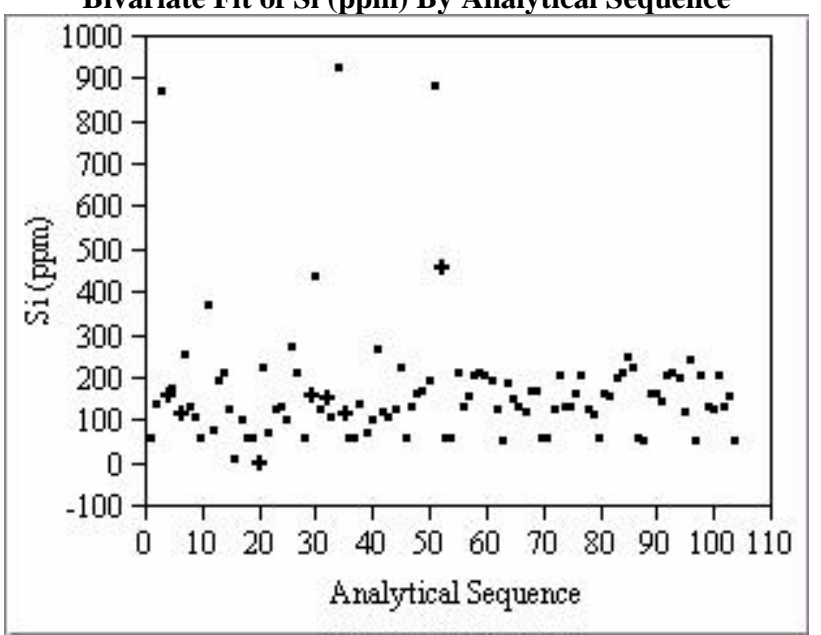


Exhibit E15. PCTs for Glasses from Underwashed Sludge in Analytical Sequence (without results for EA and Blanks)

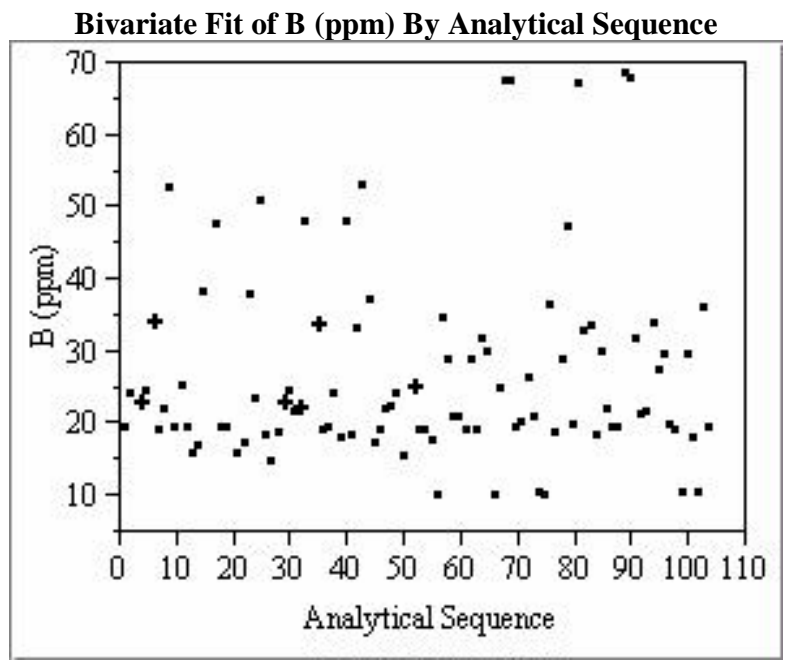

Bivariate Fit of Li (ppm) By Analytical Sequence

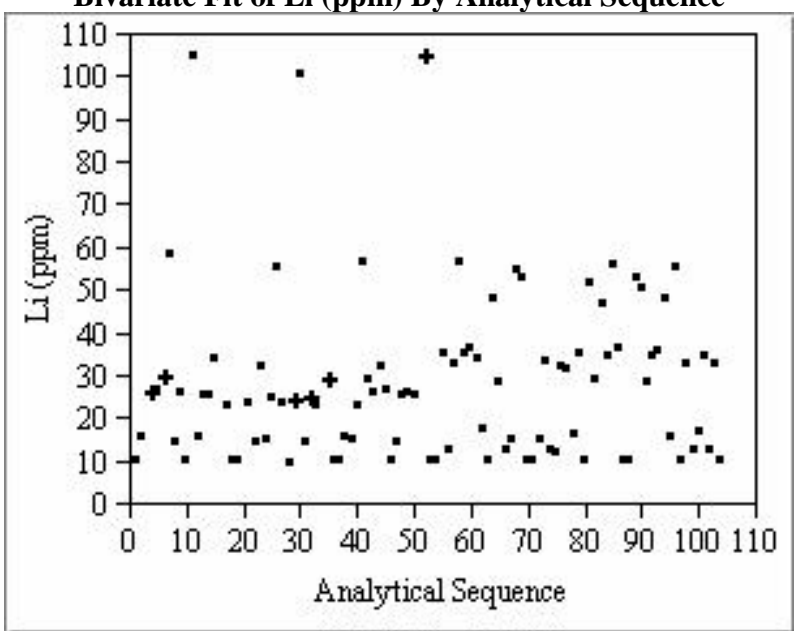

Bivariate Fit of Na (ppm) By Analytical Sequence

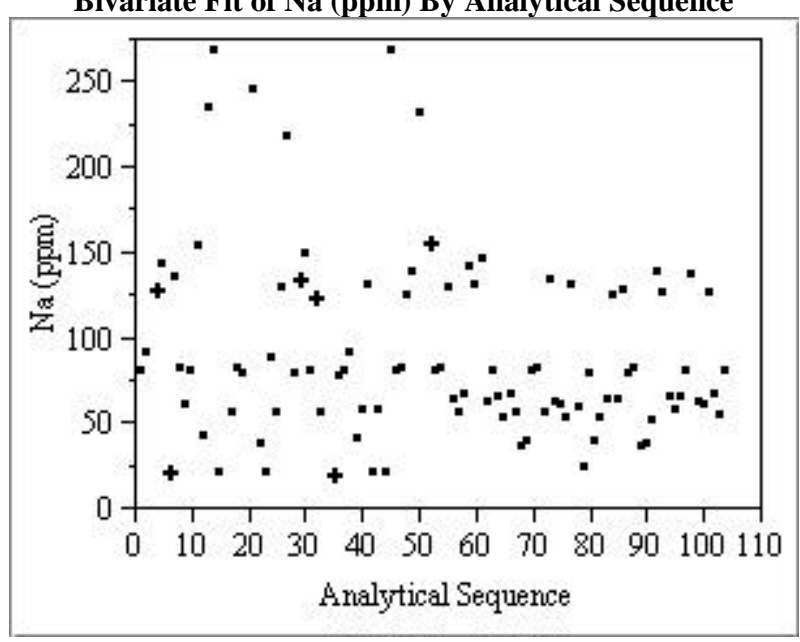

Bivariate Fit of Si (ppm) By Analytical Sequence

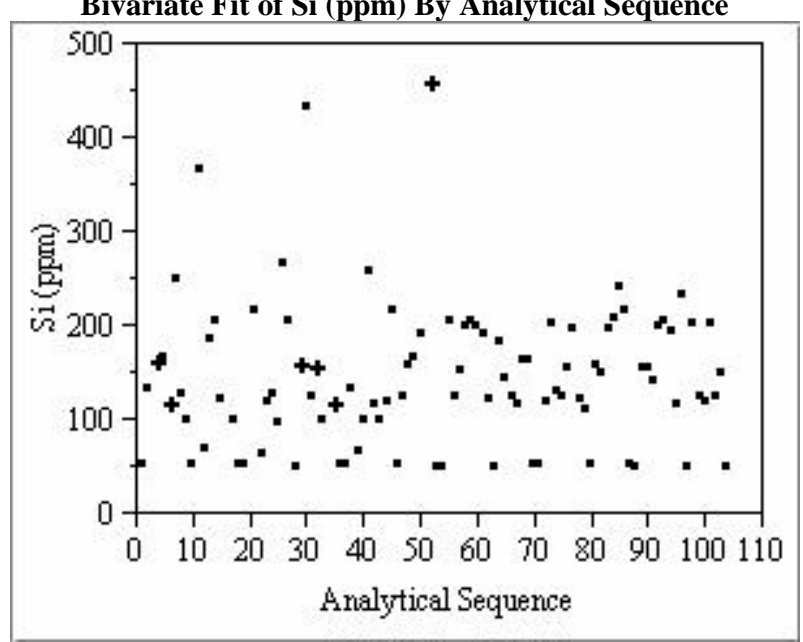




\section{Exhibit E16. PCTs by Glass ID (Including EA and Blanks) for Underwashed Sludge}

Oneway Analysis of B (ppm) By Glass ID (shortened)

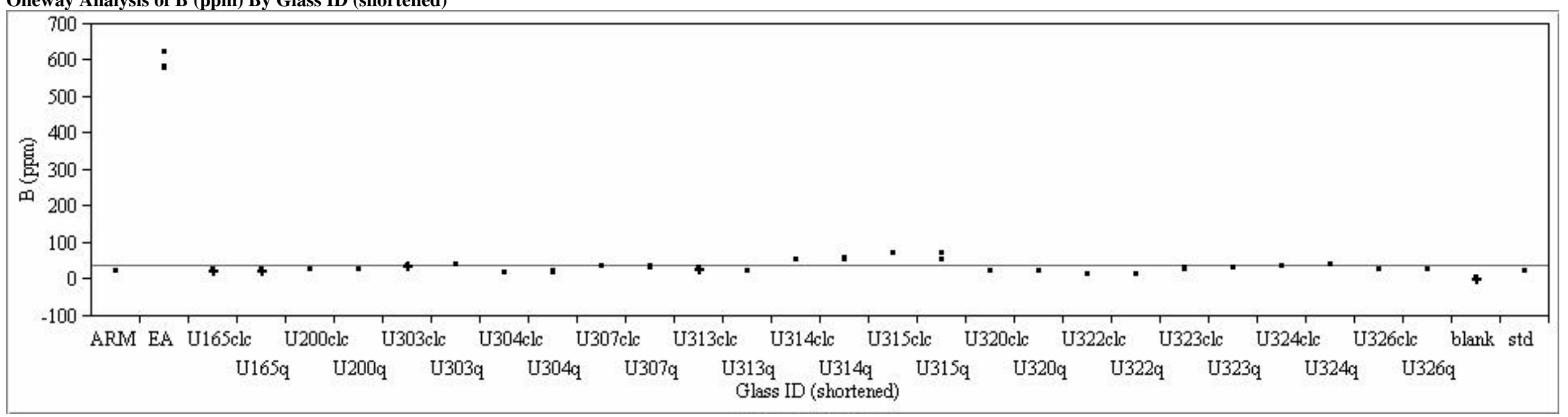

Oneway Analysis of Li (ppm) By Glass ID (shortened)

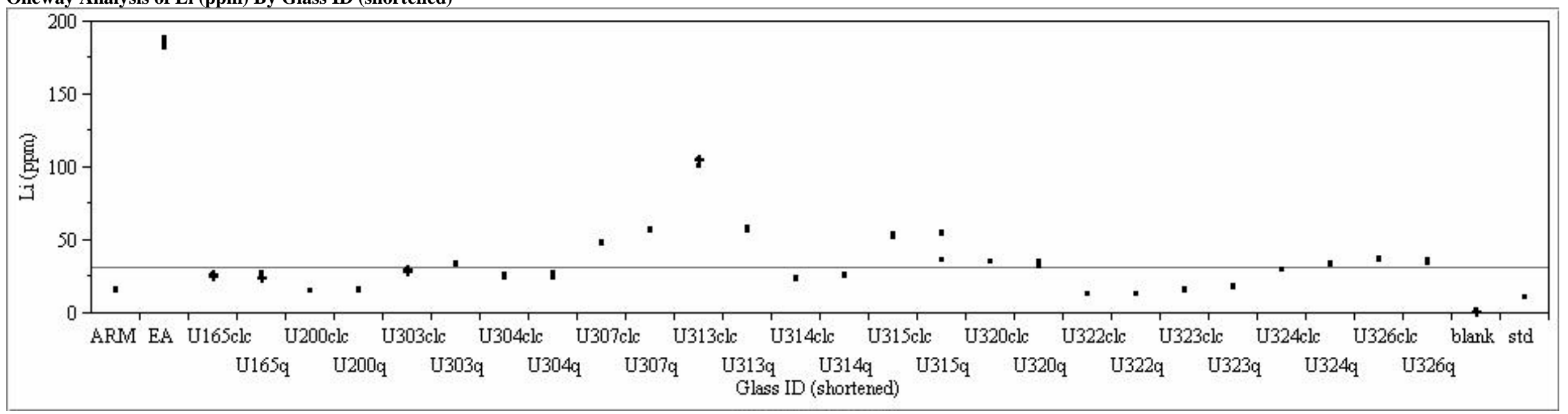




\section{Exhibit E16. PCTs by Glass ID (Including EA and Blanks) for Underwashed Sludge (continued)}

Oneway Analysis of Na (ppm) By Glass ID (shortened)

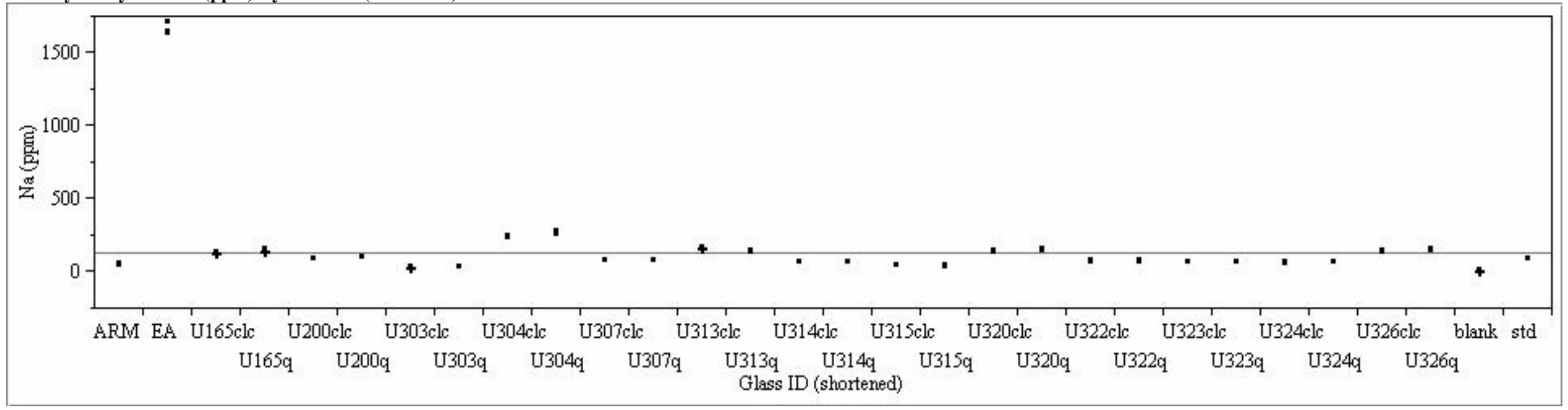

Oneway Analysis of Si (ppm) By Glass ID (shortened)

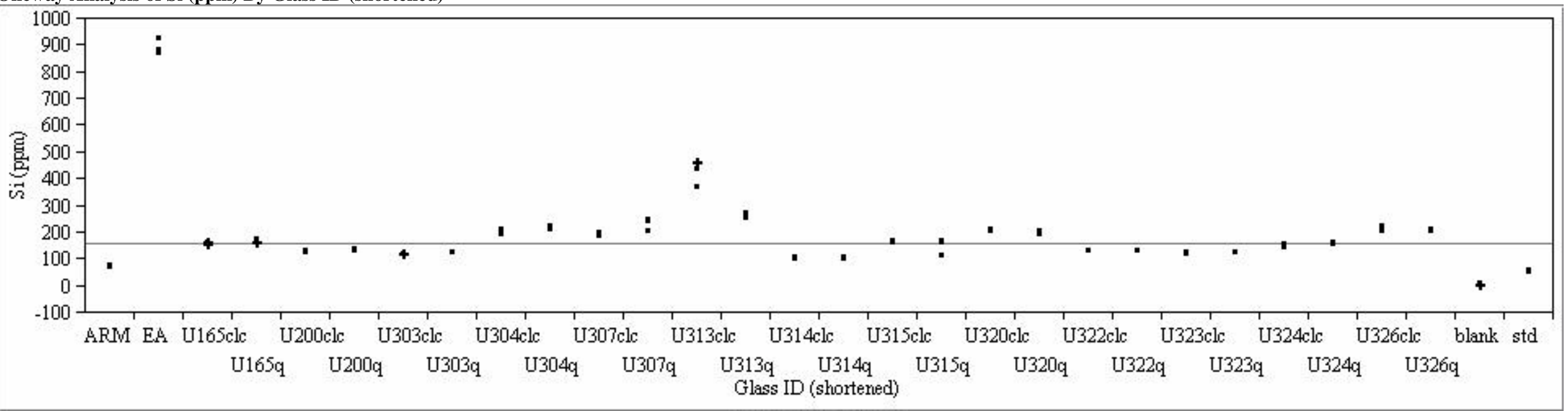




\section{Exhibit E17. PCTs by Glass ID (Excluding EA and Blanks) for Underwashed Sludge}

Oneway Analysis of B (ppm) By Glass ID (shortened)

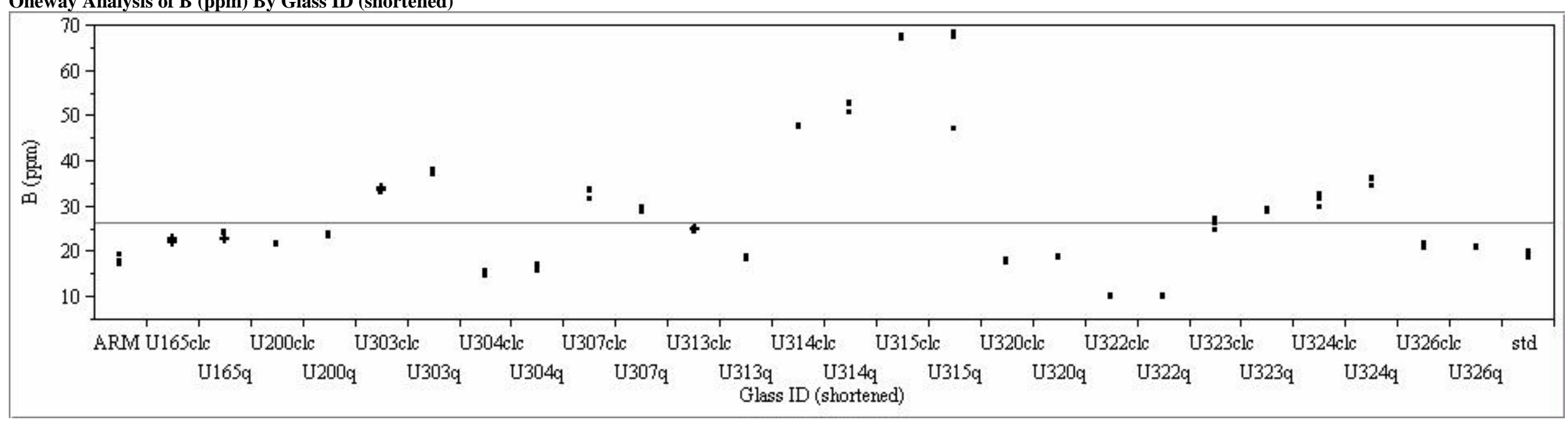

Oneway Analysis of Li (ppm) By Glass ID (shortened)

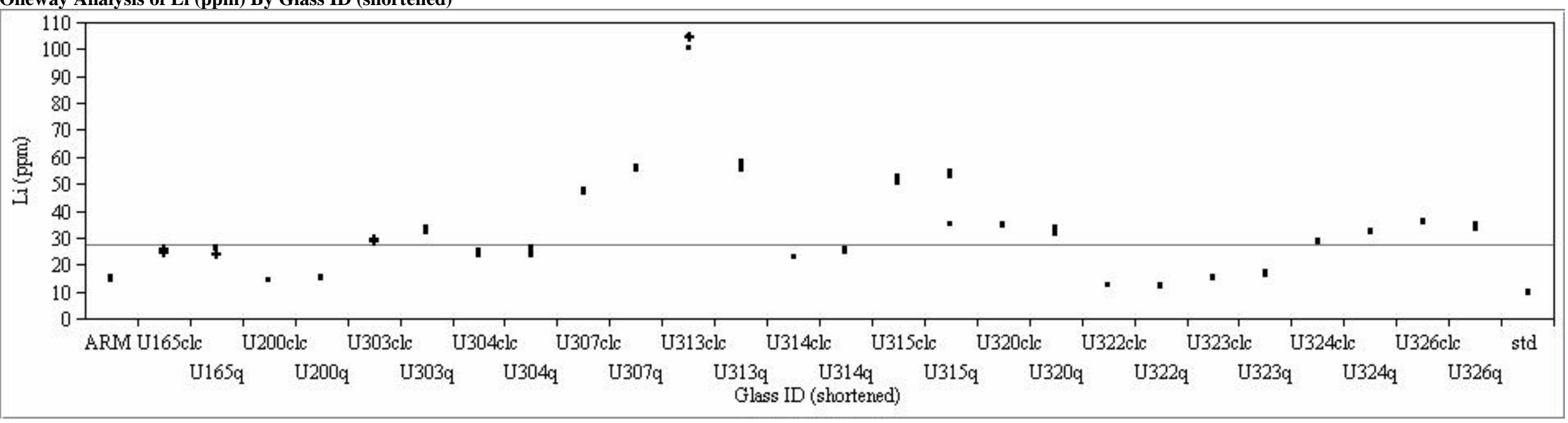


Exhibit E17. PCTs by Glass ID (Excluding EA and Blanks) for Underwashed Sludge (continued)

Oneway Analysis of Na (ppm) By Glass ID (shortened)

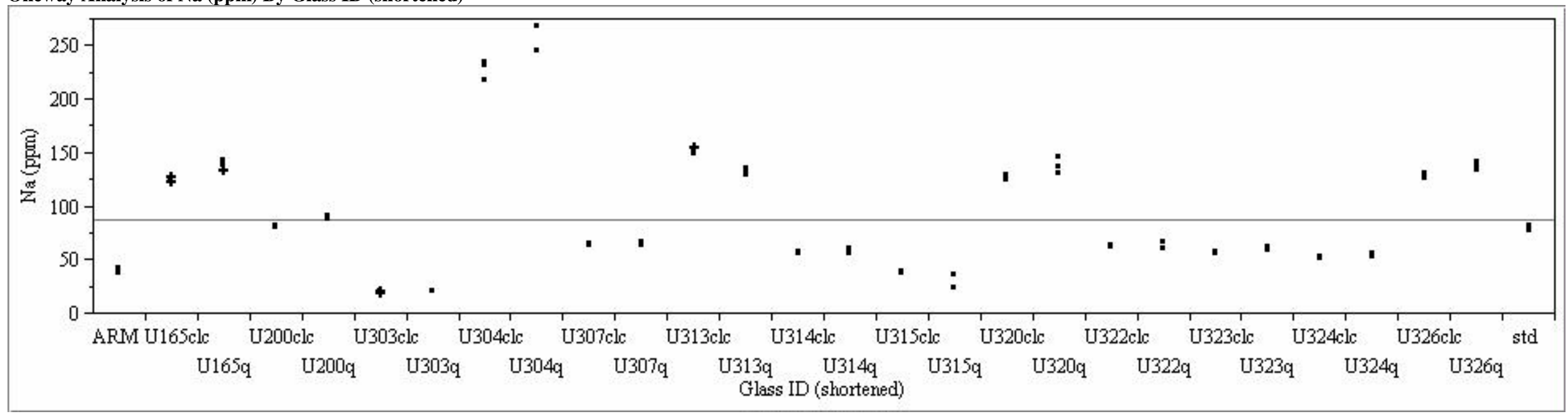

Oneway Analysis of Si (ppm) By Glass ID (shortened)

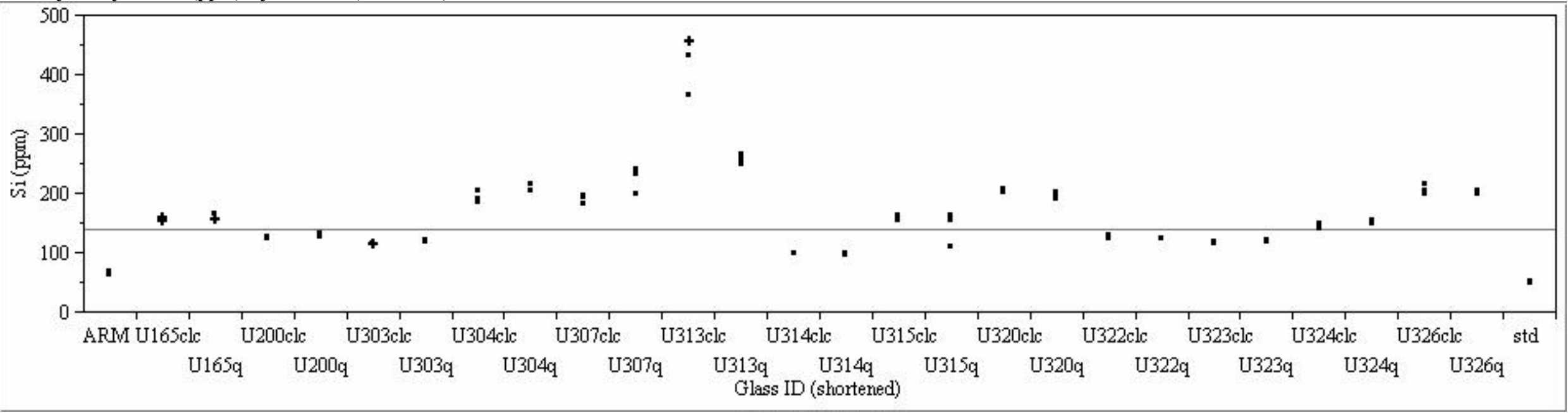




\section{Exhibit E18. Measurements of the Solutions Standards by ICP Block for Underwashed Sludge Case}

Oneway Analysis of B (ppm) By Block

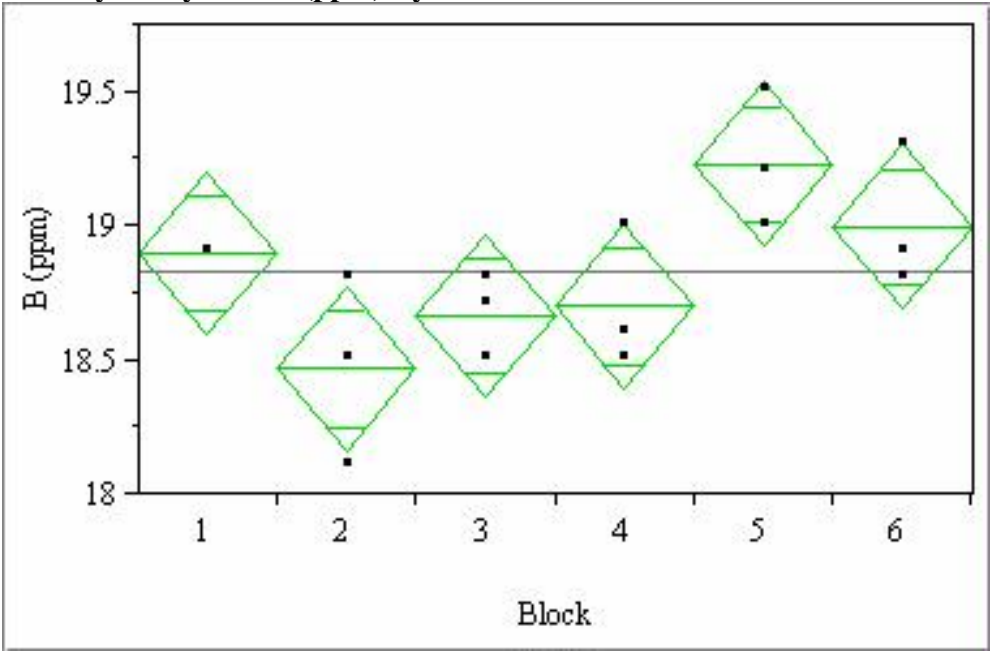

\section{Oneway Anova}

\section{Summary of Fit}

Rsquare

Adj Rsquare

0.614561

0.453961

Root Mean Square Error $\quad 0.241523$

Mean of Response 18.82778

Observations (or Sum Wgts)

$$
18
$$

Analysis of Variance

Source DF Sum of Squares Mean Square F Ratio Prob > F

$\begin{array}{lrllll}\text { Block } & 5 & 1.1161111 & 0.223222 & 3.8267 & 0.0264\end{array}$

$\begin{array}{llll}\text { Error } & 12 & 0.7000000 & 0.058333\end{array}$

C. Total $17 \quad 1.816111$

Means for Oneway Anova

$\begin{array}{lrrrrr}\text { Level } & \text { Number } & \text { Mean } & \text { Std Error } & \text { Lower 95\% } & \text { Upper 95\% } \\ 1 & 3 & 18.9000 & 0.13944 & 18.596 & 19.204 \\ 2 & 3 & 18.4667 & 0.13944 & 18.163 & 18.770 \\ 3 & 3 & 18.6667 & 0.13944 & 18.363 & 18.970 \\ 4 & 3 & 18.7000 & 0.13944 & 18.396 & 19.004 \\ 5 & 3 & 19.2333 & 0.13944 & 18.930 & 19.537 \\ 6 & 3 & 19.0000 & 0.13944 & 18.696 & 19.304\end{array}$

Std Error uses a pooled estimate of error variance
Oneway Analysis of $\mathrm{Li}$ (ppm) By Block

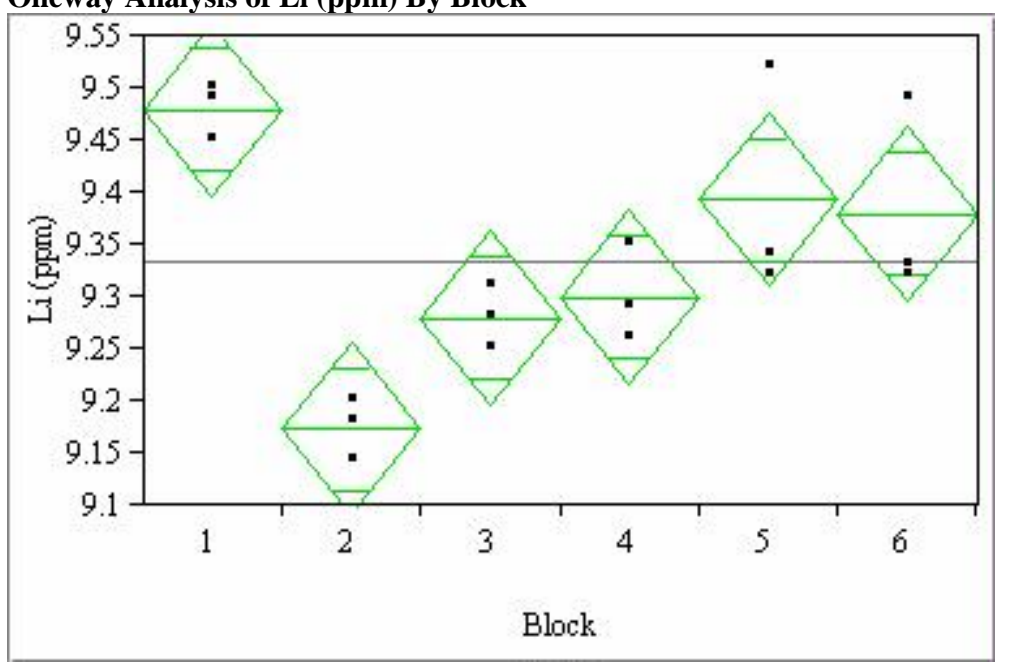

\section{Oneway Anova}

Summary of Fit

Rsquare

0.767223
0.670233

Adj Rsquare

\begin{tabular}{ll} 
Root Mean Square Error $\quad 0.065659$ \\
\hline
\end{tabular}

Mean of Response

9.334444

Observations (or Sum Wgts)

Analysis of Variance

Source DF Sum of Squares Mean Square F Ratio Prob > F

$\begin{array}{llllll}\text { Block } & 5 & 0.17051111 & 0.034102 & 7.9103 & 0.0017\end{array}$

$\begin{array}{lll}\text { Error } & 12 & 0.05173333\end{array}$

0.004311

C. Total $17 \quad 0.22224444$

Means for Oneway Anova

Level Number Mean Std Error Lower 95\% Upper 95\%

$\begin{array}{llllll}1 & 3 & 9.48000 & 0.03791 & 9.3974 & 9.5626\end{array}$

$\begin{array}{llllll}2 & 3 & 9.17333 & 0.03791 & 9.0907 & 9.2559\end{array}$

$\begin{array}{llllll}3 & 3 & 9.28000 & 0.03791 & 9.1974 & 9.3626\end{array}$

$\begin{array}{llllll}4 & 3 & 9.30000 & 0.03791 & 9.2174 & 9.3826 \\ 5 & 3 & 9.39333 & 0.03791 & 9.3107 & 9.4759\end{array}$

$\begin{array}{llllll}6 & 3 & 9.38000 & 0.03791 & 9.2974 & 9.4626\end{array}$

Std Error uses a pooled estimate of error variance 


\section{Exhibit E18. Measurements of the Solutions Standards by ICP Block for Underwashed Sludge Case (continued)}

\section{Oneway Analysis of Na (ppm) By Block}

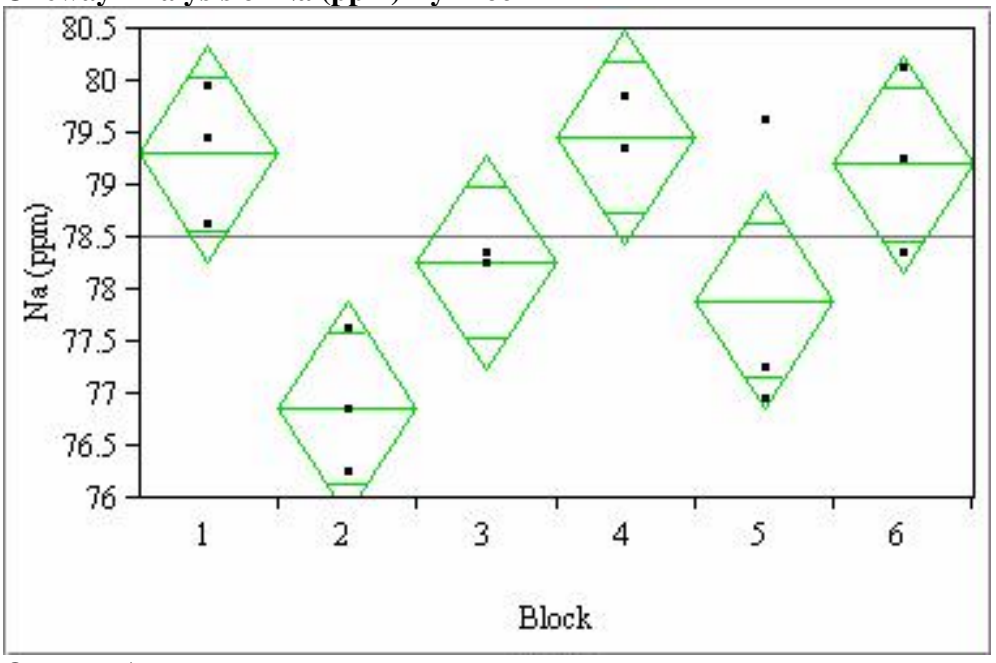

\section{Oneway Anova \\ Summary of Fit}

Rsquare

Adj Rsquare

Root Mean Square Error $\quad 0.817517$

Mean of Response $\quad 78.5$

Observations (or Sum Wgts)

Analysis of Variance

Source DF Sum of Squares Mean Square F Ratio Prob > F

$\begin{array}{lrrrrr}\text { Block } & 5 & 15.440000 & 3.08800 & 4.6204 & 0.0139\end{array}$

$\begin{array}{llll}\text { Error } & 12 & 8.020000 & 0.66833\end{array}$

$\begin{array}{lll}\text { C. Total } & 17 & 23.460000\end{array}$

Means for Oneway Anova

$\begin{array}{lrrrrr}\text { Level } & \text { Number } & \text { Mean } & \text { Std Error } & \text { Lower 95\% } & \text { Upper 95\% } \\ 1 & 3 & 79.3000 & 0.47199 & 78.272 & 80.328 \\ 2 & 3 & 76.8667 & 0.47199 & 75.838 & 77.895 \\ 3 & 3 & 78.2667 & 0.47199 & 77.238 & 79.295 \\ 4 & 3 & 79.4667 & 0.47199 & 78.438 & 80.495 \\ 5 & 3 & 77.9000 & 0.47199 & 76.872 & 78.928 \\ 6 & 3 & 79.2000 & 0.47199 & 78.172 & 80.228\end{array}$

Std Error uses a pooled estimate of error variance
Oneway Analysis of Si (ppm) By Block

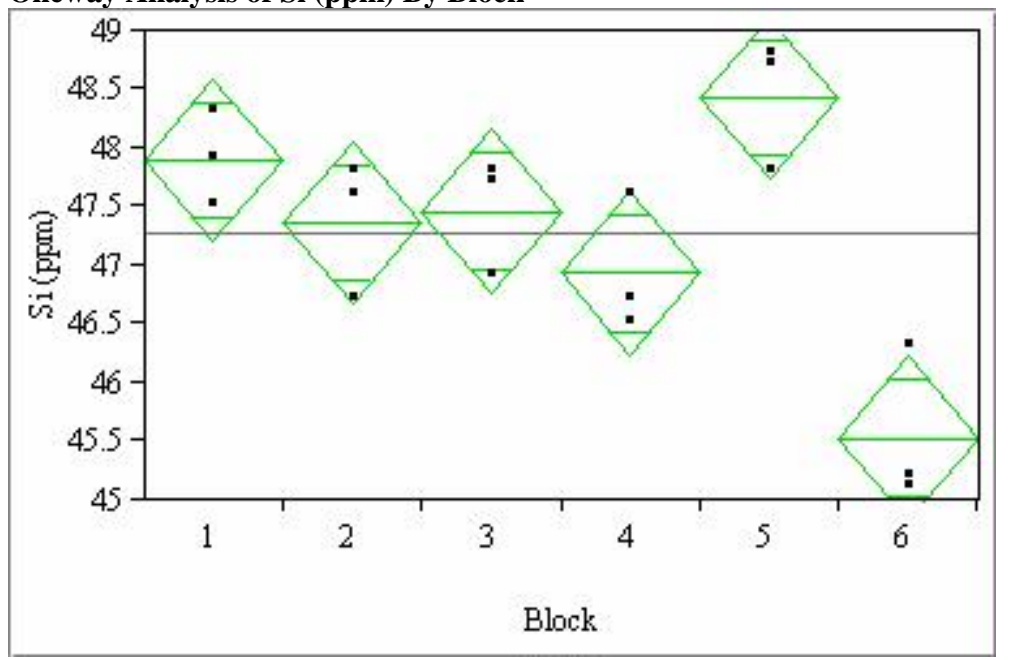

\section{Oneway Anova}

Summary of Fit

Rsquare

Adj Rsquare

0.800969

Root Mean Square

0.71804

Mean of Response

0.553273

Mean of Response

Observations (or Sum Wgts)

Analysis of Variance

Source DF Sum of Squares Mean Square F Ratio Prob > F

$\begin{array}{llllll}\text { Block } & 5 & 14.782778 & 2.95656 & 9.6584 & 0.0007\end{array}$

$\begin{array}{llll}\text { Error } & 12 & 3.673333 & 0.30611\end{array}$

C. Total $17 \quad 18.45611$

Means for Oneway Anova

Level Number Mean Std Error Lower 95\% Upper 95\%

$\begin{array}{llllll}1 & 3 & 47.9000 & 0.31943 & 47.204 & 48.596 \\ 2 & 3 & 47.3667 & 0.31943 & 46.671 & 48.063\end{array}$

$\begin{array}{llllll}2 & 3 & 47.4667 & 0.31943 & 46.671 & 48.063 \\ 3 & 3 & 46.9333 & 0.31943 & 46.771 & 48.163\end{array}$

$\begin{array}{llllll}3 & 3 & 47.4667 & 0.31943 & 46.771 & 48.163 \\ 4 & 3 & 46.9333 & 0.31943 & 46.237 & 47.629\end{array}$

$\begin{array}{llllll}5 & 3 & 48.4333 & 0.31943 & 47.737 & 49.129\end{array}$

$\begin{array}{llllll}6 & 3 & 45.5333 & 0.31943 & 44.837 & 46.229\end{array}$

Std Error uses a pooled estimate of error variance 


\section{Exhibit E19. Correlations of PCTs for Underwashed Case}

\section{Under-Measured-clc}

\section{Correlations}

$\log \mathrm{NL}[\mathrm{B}(\mathrm{g} / \mathrm{L})] \quad \log \mathrm{NL}[\mathrm{Li}(\mathrm{g} / \mathrm{L})] \quad \log \mathrm{NL}[\mathrm{Na}(\mathrm{g} / \mathrm{L})] \quad \log \mathrm{NL}[\mathrm{Si}(\mathrm{g} / \mathrm{L})]$

$\begin{array}{lrrrr}\log \mathbf{N L}[\mathbf{B}(\mathbf{g} / \mathbf{L})] & 1.0000 & 0.8999 & 0.5959 & 0.6516 \\ \log \mathbf{N L}[\mathbf{L i}(\mathbf{g} / \mathbf{L})] & 0.8999 & 1.0000 & 0.7558 & 0.8601 \\ \log \mathbf{N L}[\mathbf{N a}(\mathbf{g} / \mathbf{L})] & 0.5959 & 0.7558 & 1.0000 & 0.8566 \\ \log \mathbf{N L}[\mathbf{S i}(\mathbf{g} / \mathbf{L})] & 0.6516 & 0.8601 & 0.8566 & 1.0000\end{array}$

\section{Scattenplot Matrix}

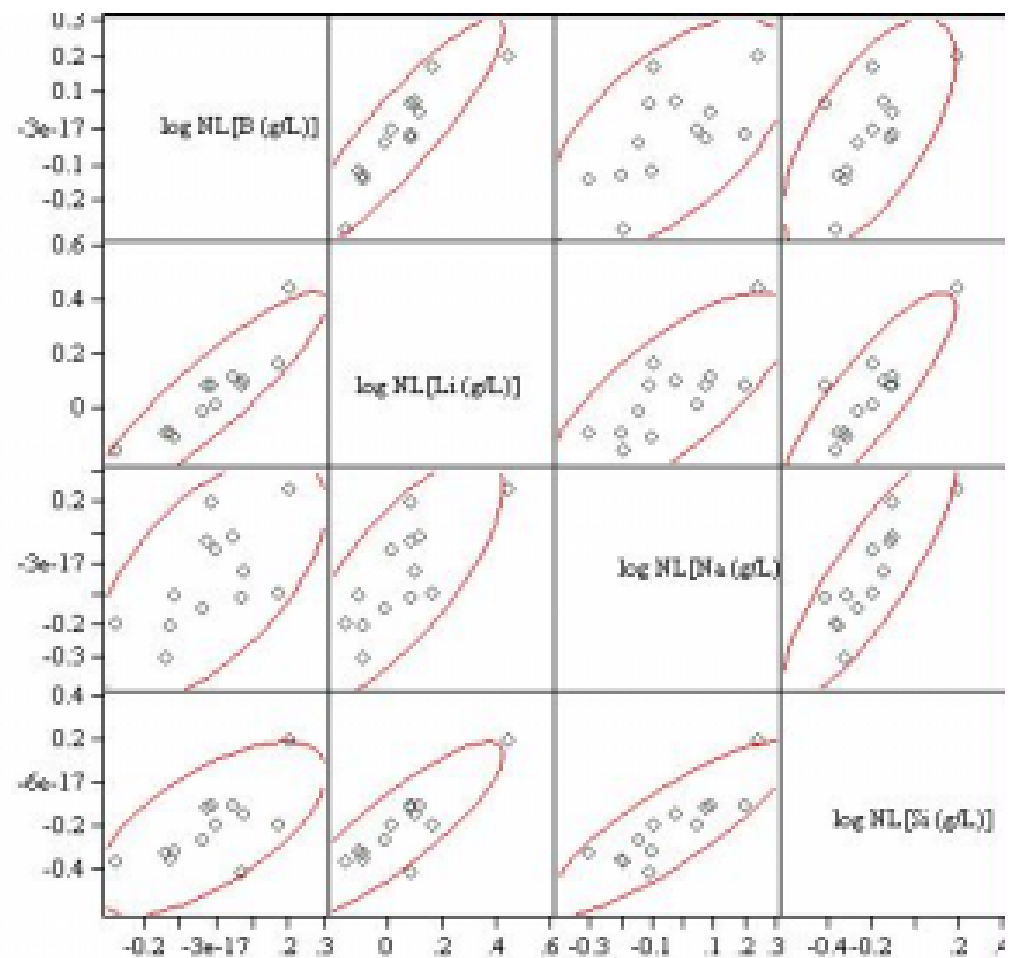

Under-Measured-quenched

\section{Correlations}

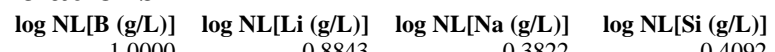

$\begin{array}{lllll}\log \mathbf{N} \mathbf{L}[\mathbf{B}(\mathrm{g} / \mathbf{L})] & 1.0000 & 0.8843 & 0.3822 & 0.4092 \\ \log \mathbf{N} \mathbf{L}[\mathbf{L i}(\mathbf{g} / \mathbf{L})] & 0.8843 & 1.0000 & 0.4988 & 0.6341 \\ \log & 0.3822 & 0.498 & 1.0000 & 0.7977\end{array}$

$\begin{array}{lllll}\log \mathbf{N L}[\mathbf{N a}(\mathbf{g} / \mathbf{L})] & 0.3822 & 0.4988 & 1.0000 & 0.7977\end{array}$

\section{Scatterplot Matnix}

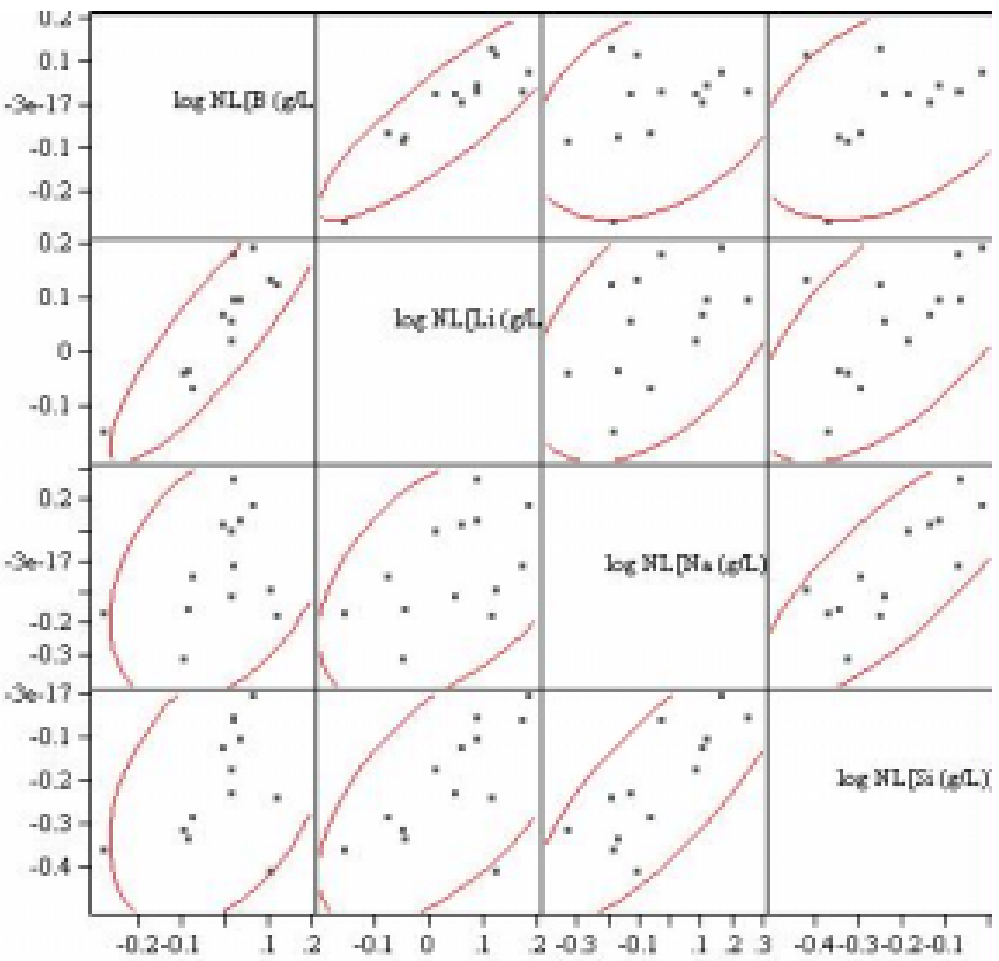


Exhibit E19. Correlations of PCTs for Underwashed Case (Continued)

\section{Under-Measured bc-clc}

\section{Correlations}

$\begin{array}{lrrrr} & \log \mathbf{N L}[\mathbf{B}(\mathbf{g} / \mathbf{L})] & \log \mathbf{N L}[\mathbf{L i}(\mathbf{g} / \mathbf{L})] & \log \mathbf{N L}[\mathbf{N a}(\mathbf{g} / \mathbf{L})] & \log \mathbf{N L}[\mathbf{S i}(\mathbf{g} / \mathbf{L})] \\ \log \mathbf{N L}[\mathbf{B}(\mathbf{g} / \mathbf{L})] & 1.0000 & 0.9056 & 0.6033 & 0.6490 \\ \log \mathbf{N L}[\mathbf{L i}(\mathbf{g} / \mathbf{L})] & 0.9056 & 1.0000 & 0.7535 & 0.8535 \\ \log \mathbf{L L}[\mathbf{N a}(\mathbf{g} / \mathbf{L})] & 0.6033 & 0.7535 & 1.0000 & 0.8551 \\ \log \mathbf{N L}[\mathbf{S i}(\mathrm{g} / \mathbf{L})] & 0.6490 & 0.8535 & 0.8551 & 1.0000\end{array}$

\section{Scattenplot Matrix}

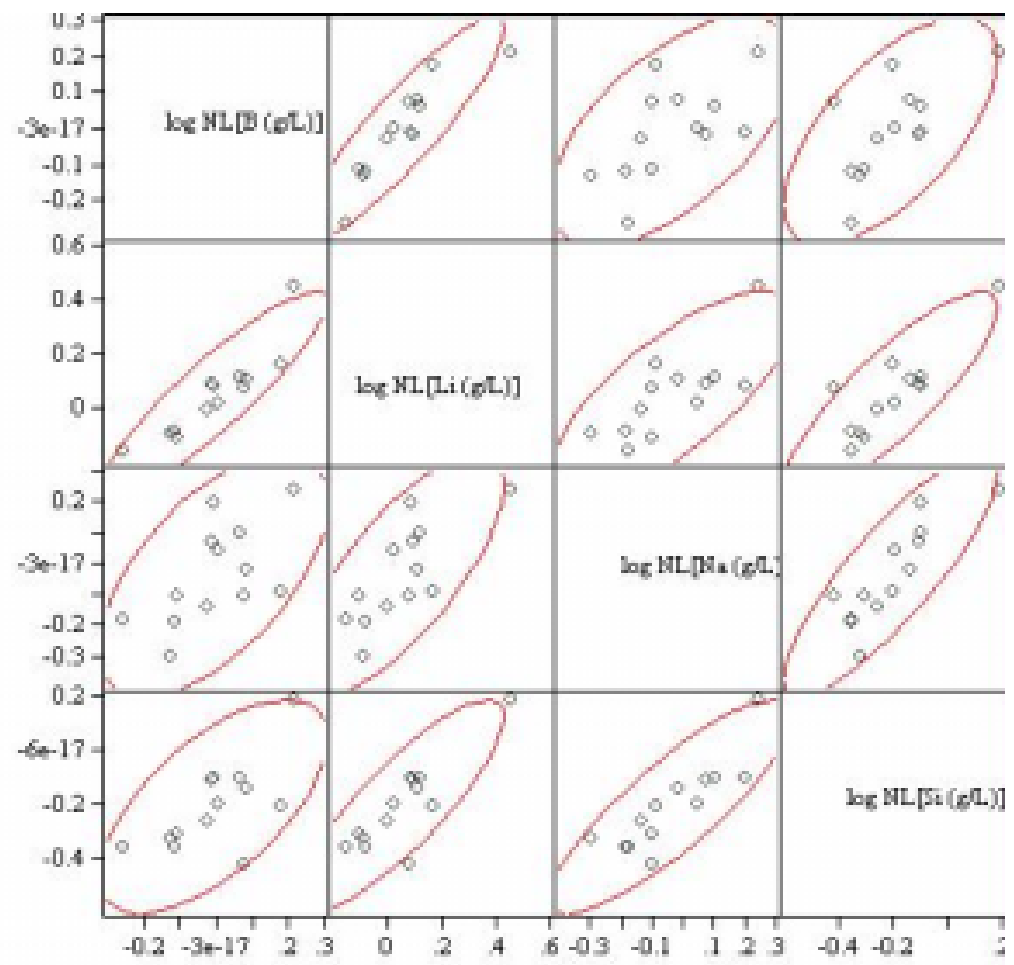

Under-Measured bc-quenched

\section{Correlations}

$\log \mathbf{N L}[\mathbf{B}(\mathrm{g} / \mathrm{L})] \quad \log \mathrm{NL}[\mathrm{Li}(\mathrm{g} / \mathrm{L})] \quad \log \mathrm{NL}[\mathrm{Na}(\mathrm{g} / \mathrm{L})] \quad \log \mathrm{NL}[\mathrm{Si}(\mathrm{g} / \mathrm{L})]$

$\begin{array}{lrrrr}\log \mathbf{N L}[\mathbf{B}(\mathbf{g} / \mathbf{L})] & 1.0000 & 0.8862 & 0.3887 & 0.4000 \\ \log \mathbf{N L} \mathbf{L i}(\mathbf{g} / \mathbf{L})] & 0.8862 & 1.0000 & 0.4977 & 0.6233 \\ \log \mathbf{N L}[\mathbf{N a}(\mathbf{g} / \mathbf{L})] & 0.3887 & 0.4977 & 1.0000 & 0.7964 \\ \log \mathbf{N L}[\mathbf{S i}(\mathbf{g} / \mathbf{L})] & 0.4000 & 0.6233 & 0.7964 & 1.0000\end{array}$

\section{Scattenplot Mahix}

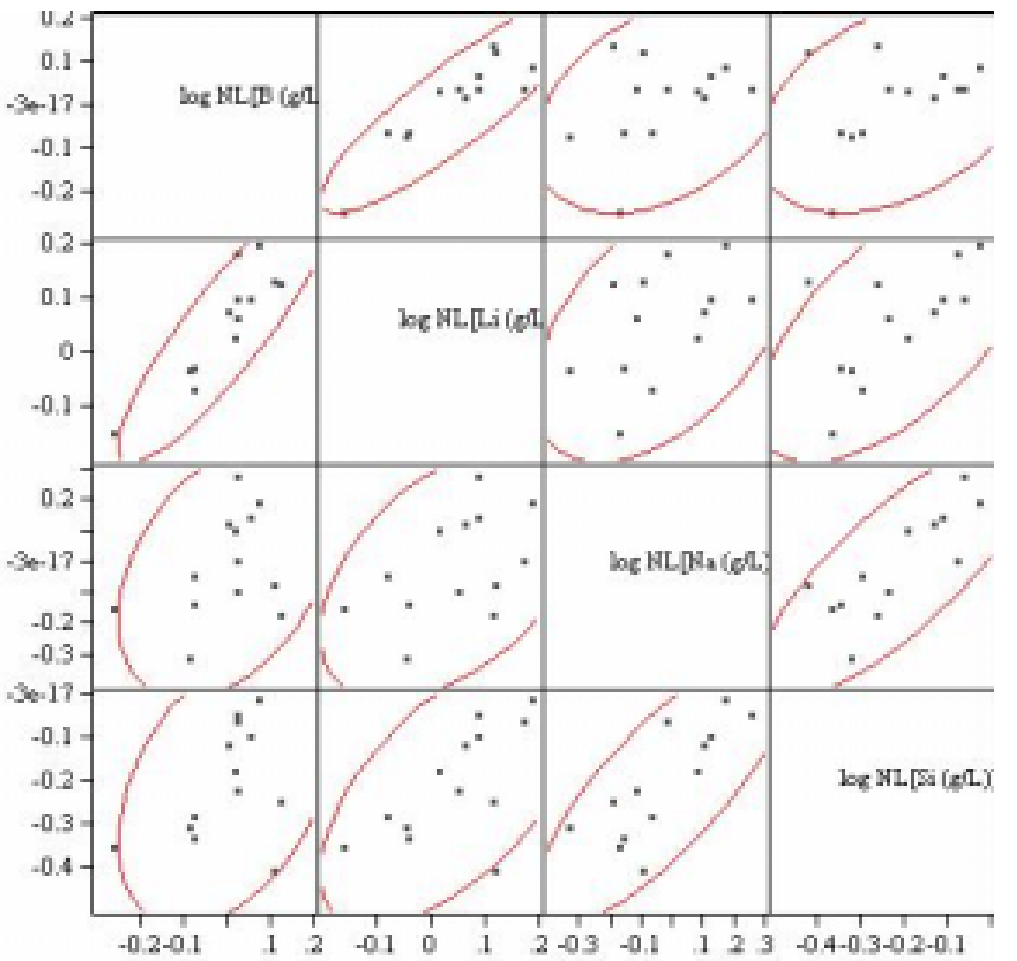


Exhibit AE19. Correlations of PCTs for Underwashed Case (Continued)

\section{Under-Targeted-clc}

\section{Correlations}

$\log \mathrm{NL}[\mathrm{B}(\mathrm{g} / \mathrm{L})] \quad \log \mathrm{NL}[\mathrm{Li}(\mathrm{g} / \mathrm{L})] \quad \log \mathrm{NL}[\mathrm{Na}(\mathrm{g} / \mathrm{L})] \quad \log \mathrm{NL}[\mathrm{Si}(\mathrm{g} / \mathrm{L})]$

$\begin{array}{lrrrr} & \log \mathbf{N L}[\mathbf{B}(\mathbf{g} / \mathbf{L})] & \log \mathbf{N L}[\mathbf{L i}(\mathbf{g} / \mathbf{L})] & \log \mathbf{N L}[\mathbf{N a}(\mathbf{g} / \mathbf{L})] & \log \mathbf{N L}[\mathbf{S i}(\mathbf{g} / \mathbf{L})] \\ \log \mathbf{N L}[\mathbf{B}(\mathbf{g} / \mathbf{L})] & 1.0000 & 0.9168 & 0.6828 & 0.7185 \\ \log \mathbf{N L}[\mathbf{L i}(\mathbf{g} / \mathbf{L})] & 0.9168 & 1.0000 & 0.7805 & 0.8932 \\ \log \mathbf{N L}[\mathbf{N a}(\mathbf{g} / \mathbf{L})] & 0.6828 & 0.7805 & 1.0000 & 0.8724 \\ \log \mathbf{N L}[\mathbf{S i}(\mathbf{g} / \mathbf{L})] & 0.7185 & 0.8932 & 0.8724 & 1.0000\end{array}$

\section{Scattemplot Matrix}

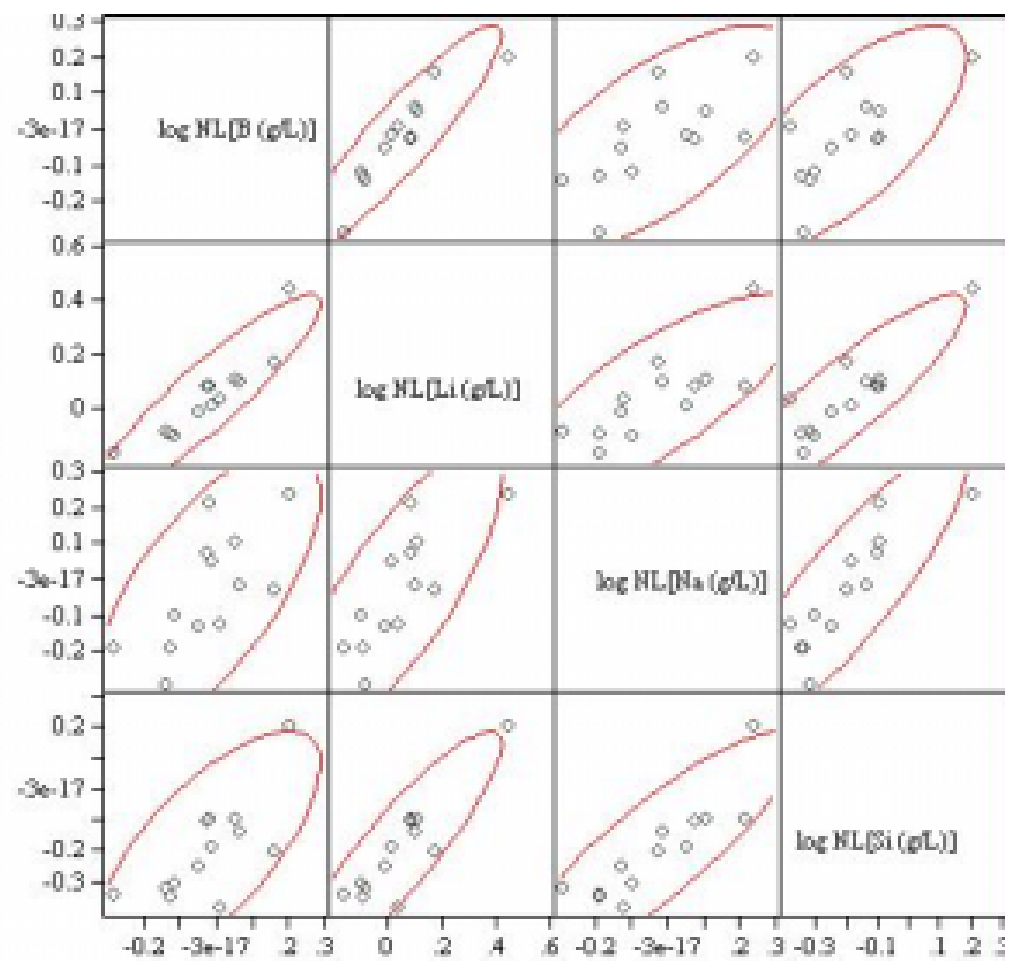

\section{Under-Targeted-quenched}

\section{Correlations}

$\log \mathrm{NL}[\mathrm{B}(\mathrm{g} / \mathrm{L})] \quad \log \mathrm{NL}[\mathrm{Li}(\mathrm{g} / \mathrm{L})] \quad \log \mathrm{NL}[\mathrm{Na}(\mathrm{g} / \mathrm{L})] \quad \log \mathrm{NL}[\mathrm{Si}(\mathrm{g} / \mathrm{L})]$

\begin{tabular}{|c|c|c|c|c|}
\hline $\log N L[B(g / L)]$ & 1.0000 & 0.8920 & 0.4913 & 0.5085 \\
\hline $\log \mathrm{NL}[\mathrm{Li}($ & 0.8920 & 1.0000 & 0.5402 & 0.7003 \\
\hline $\log \mathrm{NL}[\mathrm{Na}(\mathrm{g} / \mathrm{L})]$ & 0.4913 & 0.5402 & 1.0000 & 0.834 \\
\hline $\log \mathrm{NL}[\mathrm{Si}(\mathrm{g} / \mathrm{L})]$ & 0.5085 & 0.7003 & 0.8346 & 1.0000 \\
\hline
\end{tabular}

$\log \mathrm{NL}[\mathrm{Na}(\mathrm{g} / \mathrm{L})]$

0.5085

0.8346

0.8346

\section{Scatterplot Matnix}

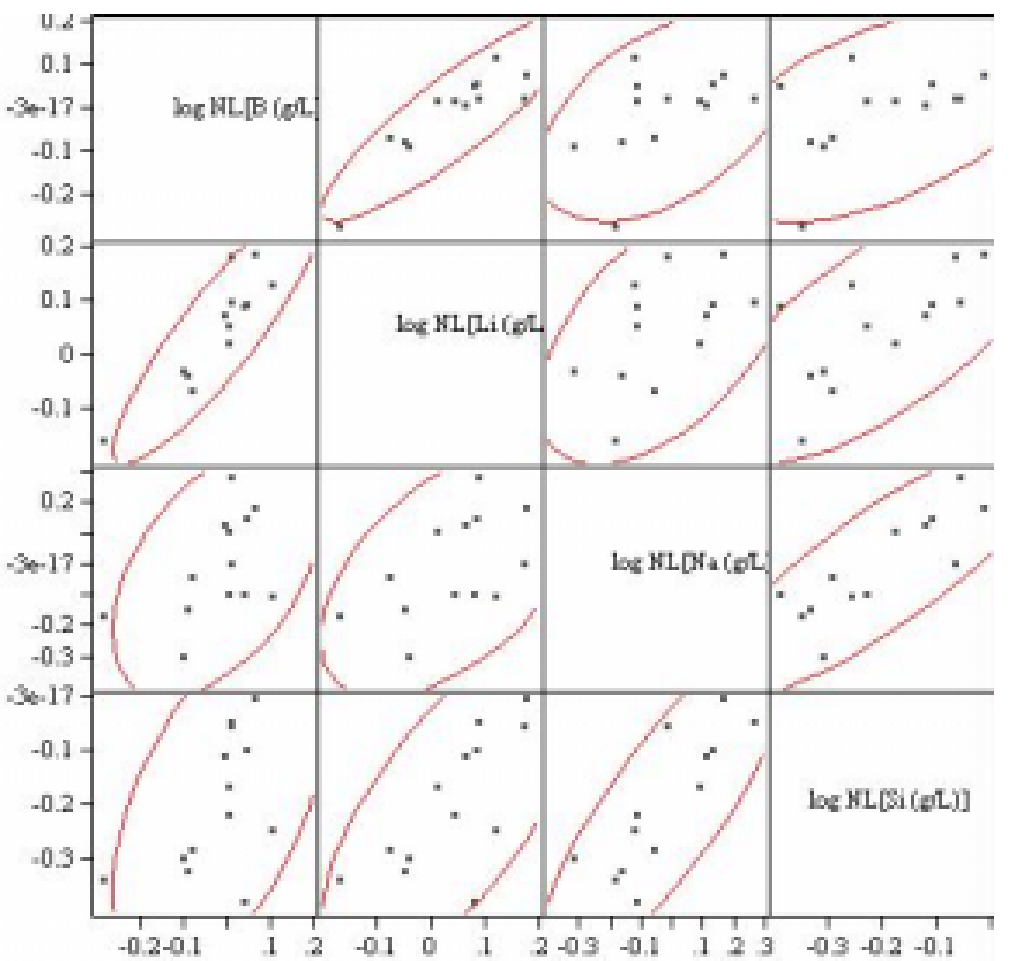


Exhibit E20. PCT vs Del Gp for Nominal-Washed Case; Measured Compositions
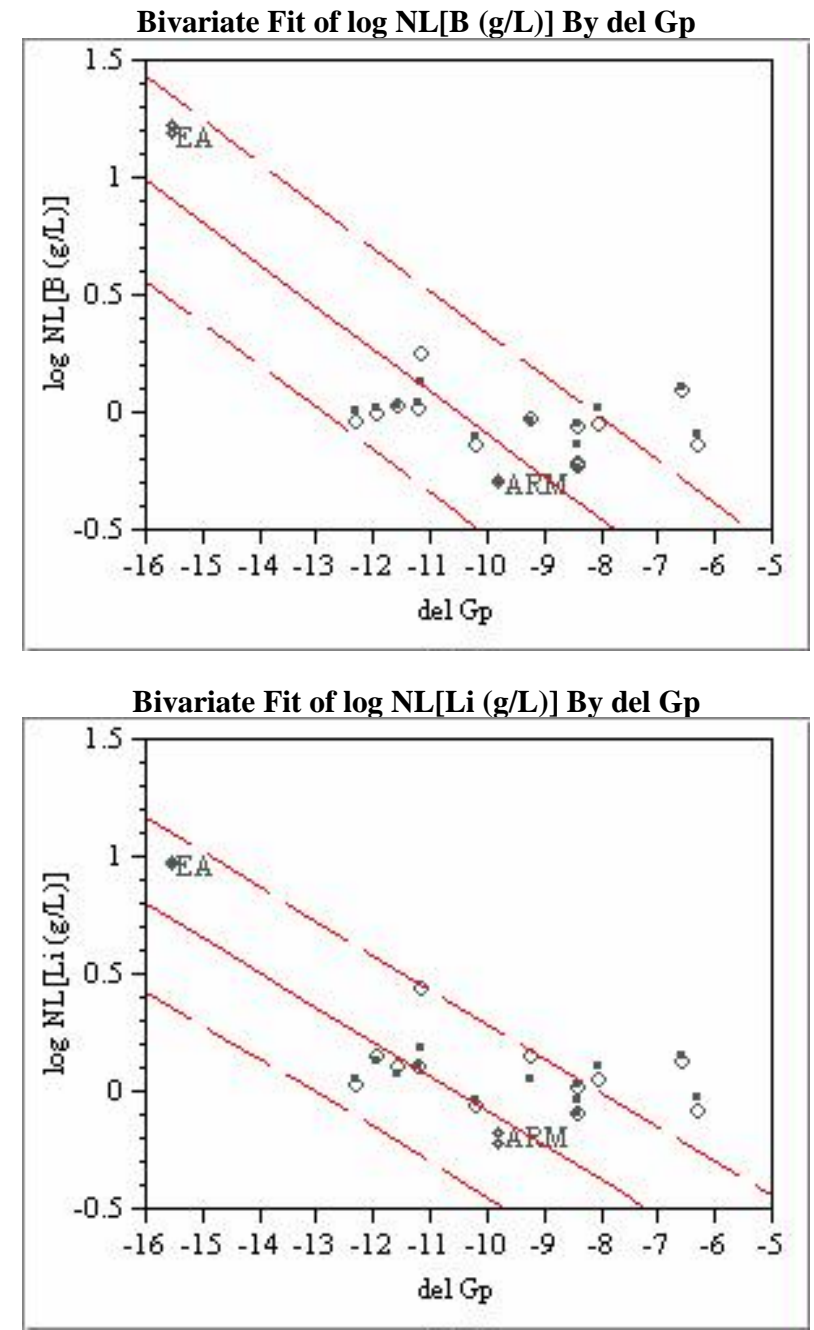

Bivariate Fit of $\log \mathrm{NL}[\mathrm{Na}(\mathrm{g} / \mathrm{L})]$ By del Gp

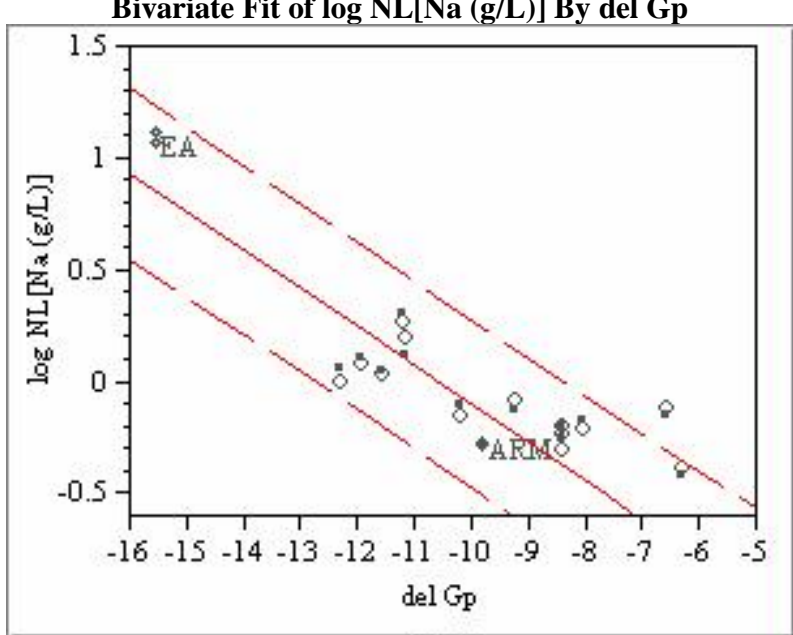

Bivariate Fit of $\log \mathrm{NL}[\mathrm{Si}(\mathrm{g} / \mathrm{L})]$ By del Gp

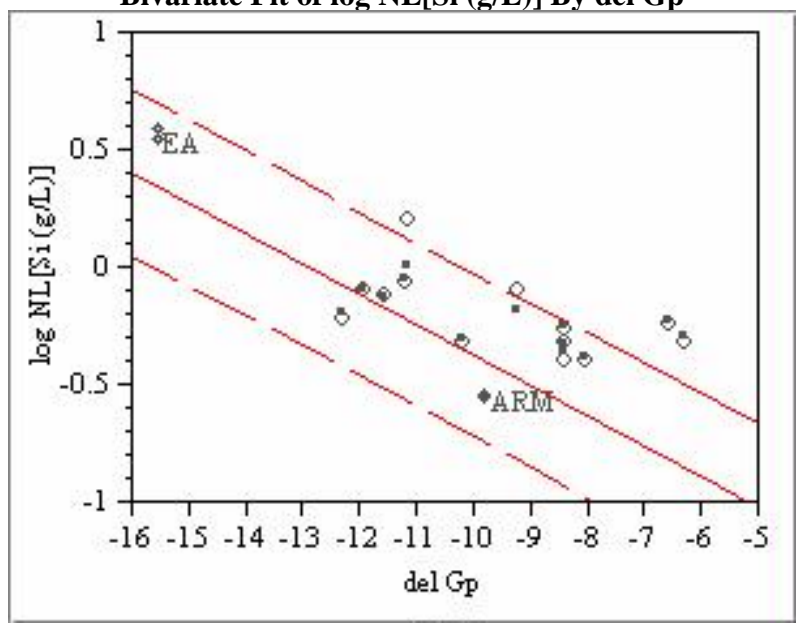


Exhibit E21. PCT vs Del Gp for Nominal-Washed Case; Measured Bias-Corrected Compositions

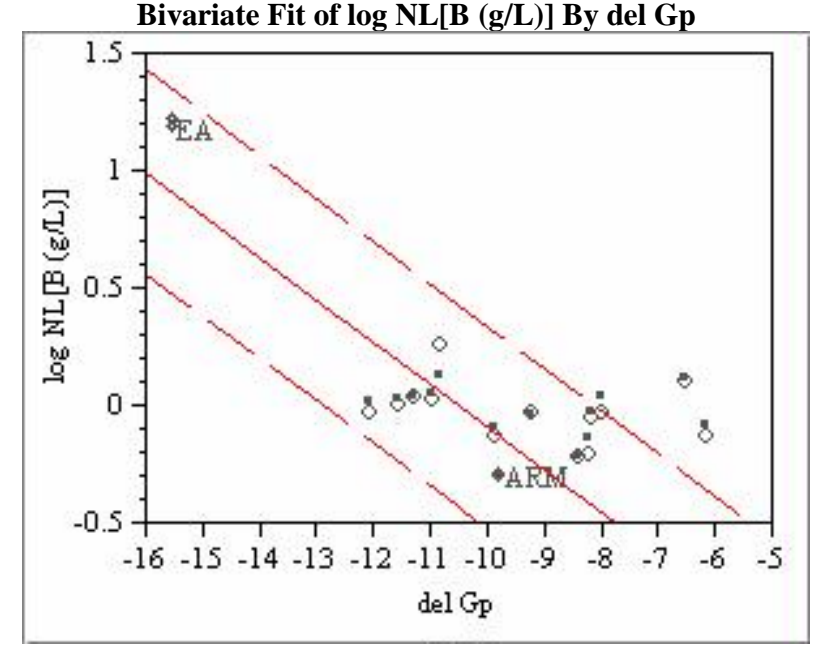

Bivariate Fit of $\log \mathrm{NL}[\mathrm{Li}(\mathrm{g} / \mathrm{L})]$ By del Gp

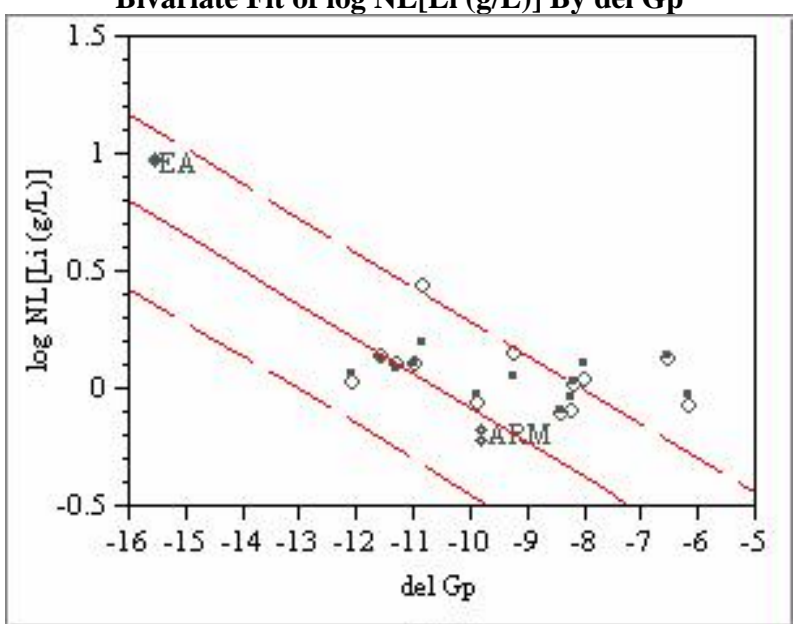

Bivariate Fit of $\log \mathrm{NL}[\mathrm{Na}(\mathrm{g} / \mathrm{L})]$ By del Gp

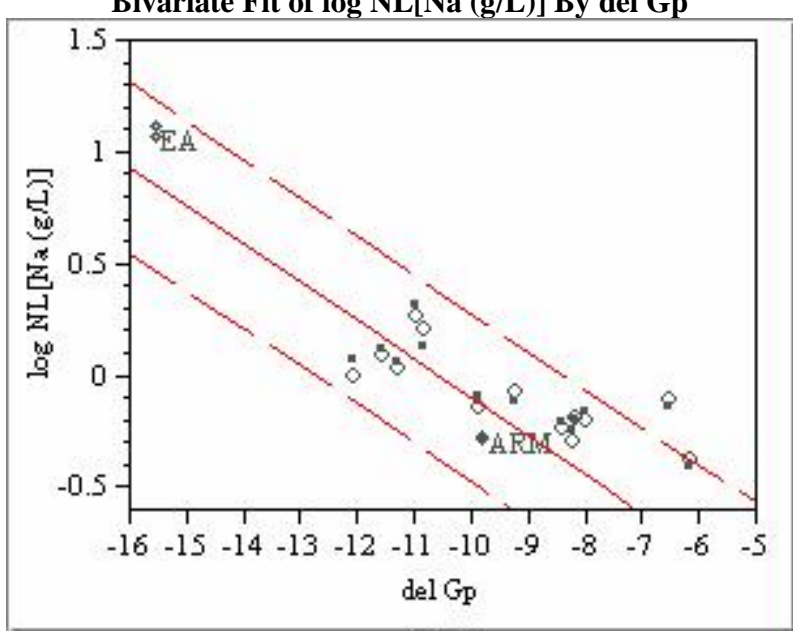

Bivariate Fit of $\log \mathrm{NL}[\mathrm{Si}(\mathrm{g} / \mathrm{L})]$ By del Gp

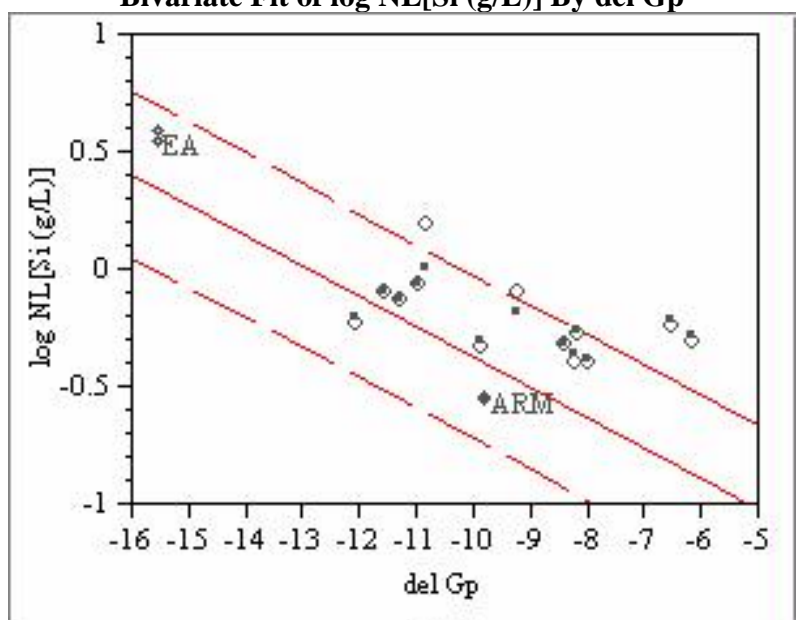


Exhibit E22. PCT vs Del Gp for Nominal-Washed Case; Targeted Compositions

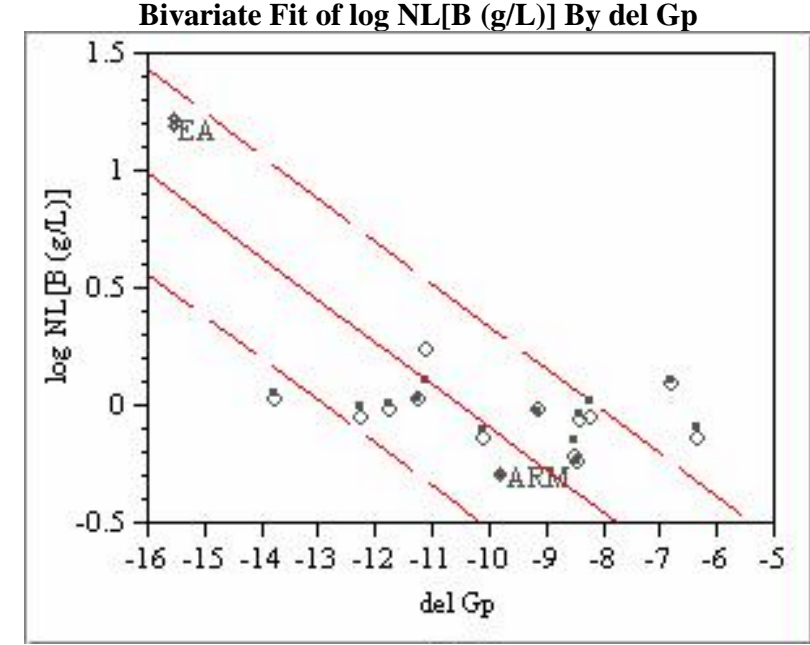

Bivariate Fit of $\log \mathrm{NL}[\mathrm{Li}(\mathrm{g} / \mathrm{L})]$ By del Gp

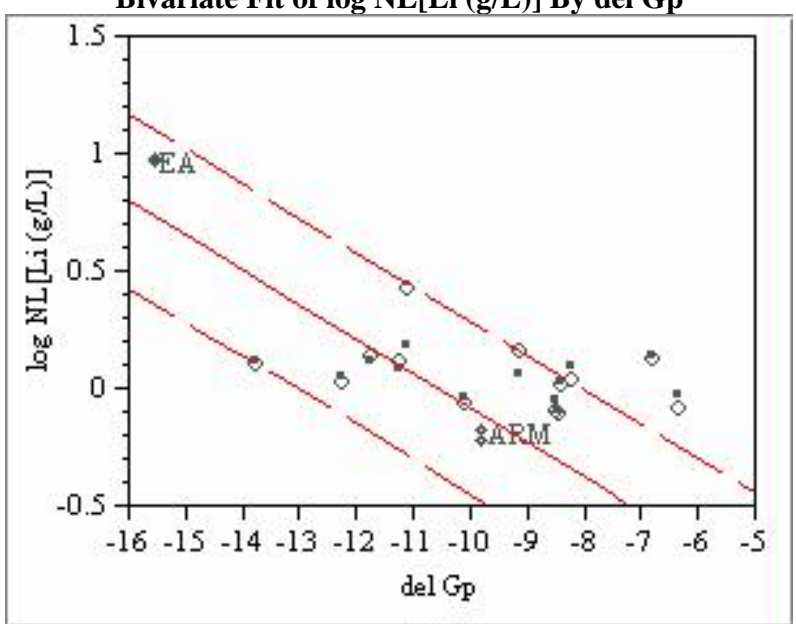

Bivariate Fit of $\log \mathrm{NL}[\mathrm{Na}(\mathrm{g} / \mathrm{L})]$ By del Gp

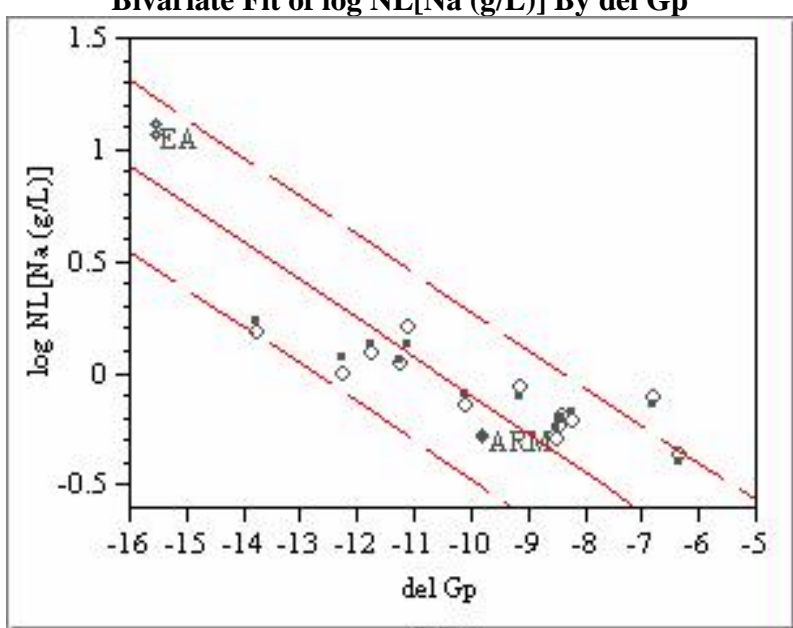

Bivariate Fit of $\log \mathrm{NL}[\mathrm{Si}(\mathrm{g} / \mathrm{L})]$ By del Gp

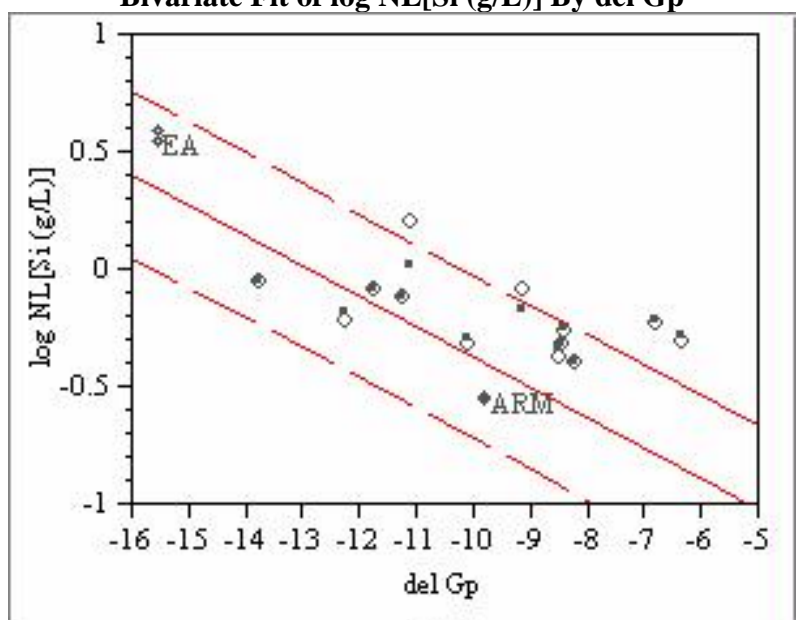


Exhibit E23. PCT vs Del Gp for Underwashed Case; Measured Compositions

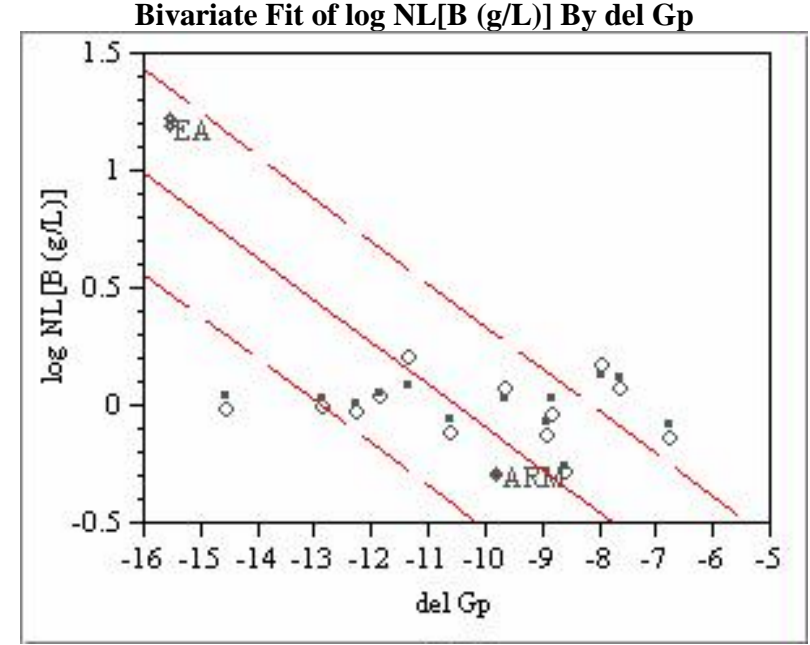

Bivariate Fit of $\log \mathrm{NL}[\mathrm{Li}(\mathrm{g} / \mathrm{L})]$ By del Gp

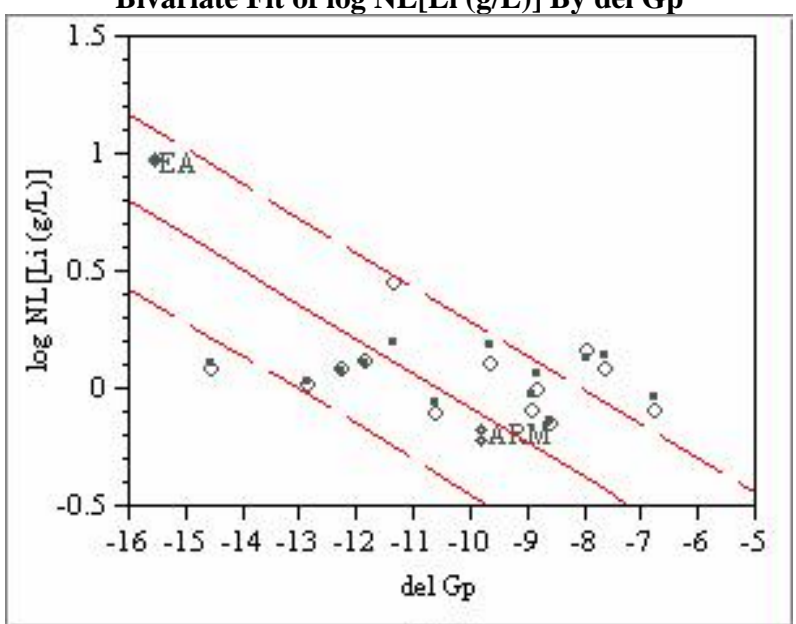

Bivariate Fit of $\log \mathrm{NL}[\mathrm{Na}(\mathrm{g} / \mathrm{L})]$ By del Gp

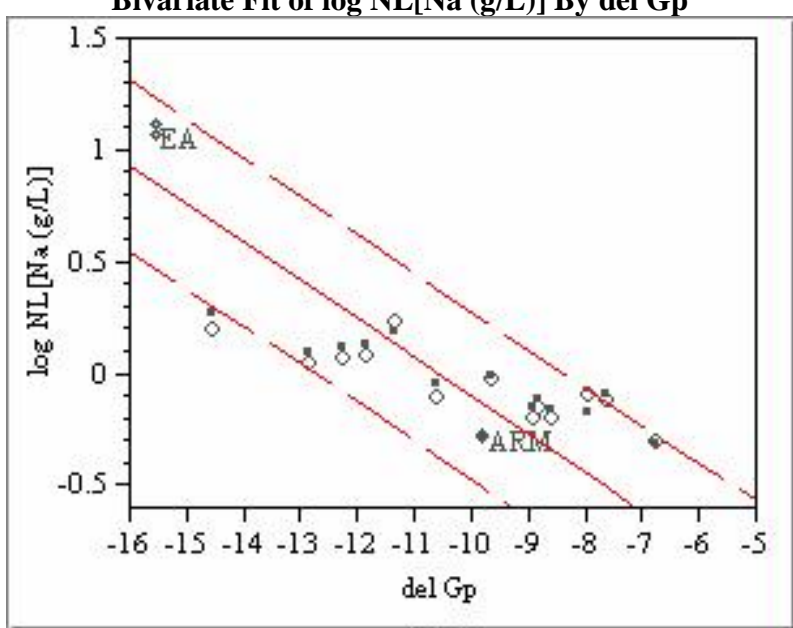

Bivariate Fit of $\log \mathrm{NL}[\mathrm{Si}(\mathrm{g} / \mathrm{L})]$ By del Gp

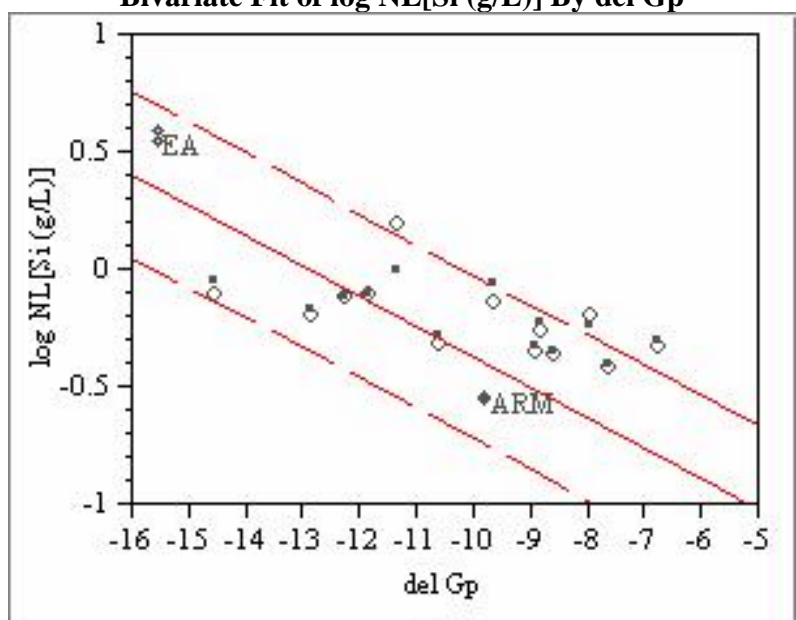


Exhibit E24. PCT vs Del Gp for Underwashed Case; Measured Bias-Corrected Compositions

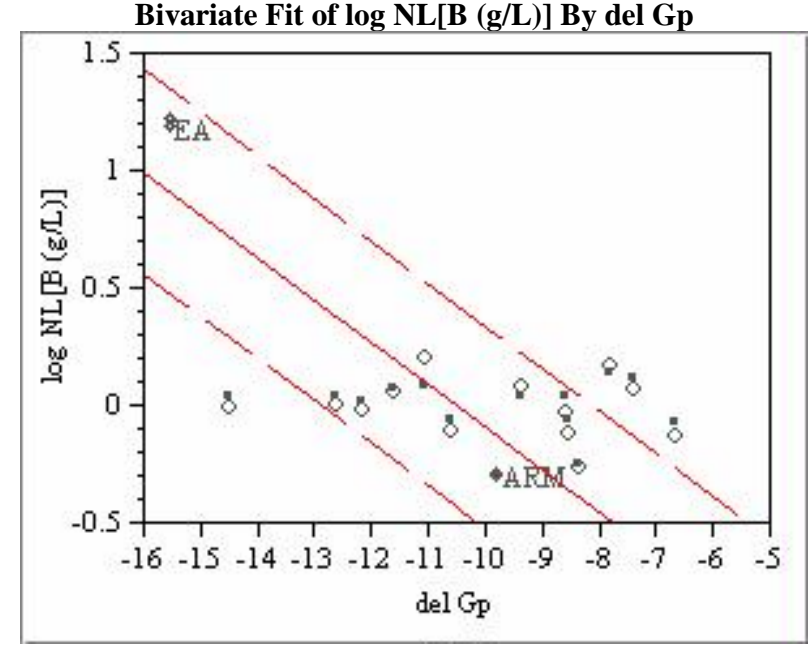

Bivariate Fit of $\log N L[L i(g / L)]$ By del Gp

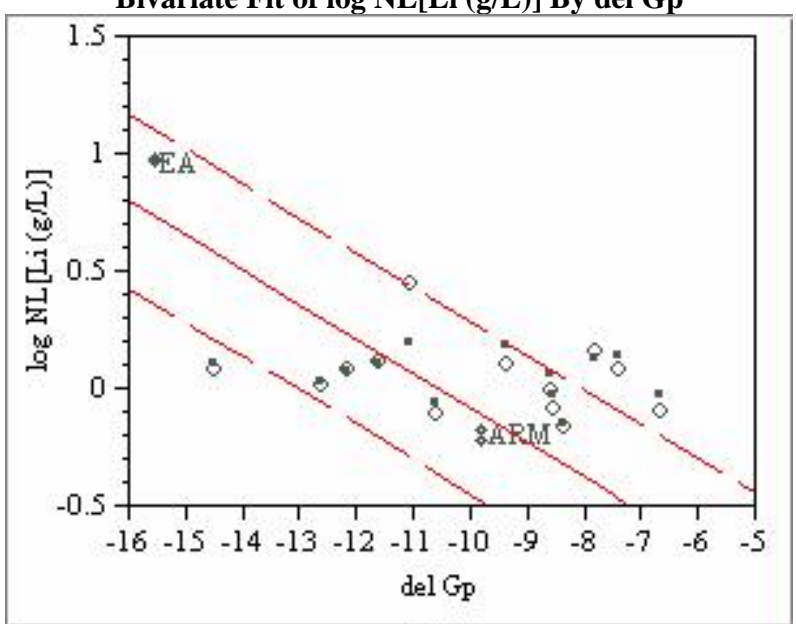

Bivariate Fit of $\log \mathrm{NL}[\mathrm{Na}(\mathrm{g} / \mathrm{L})]$ By del Gp

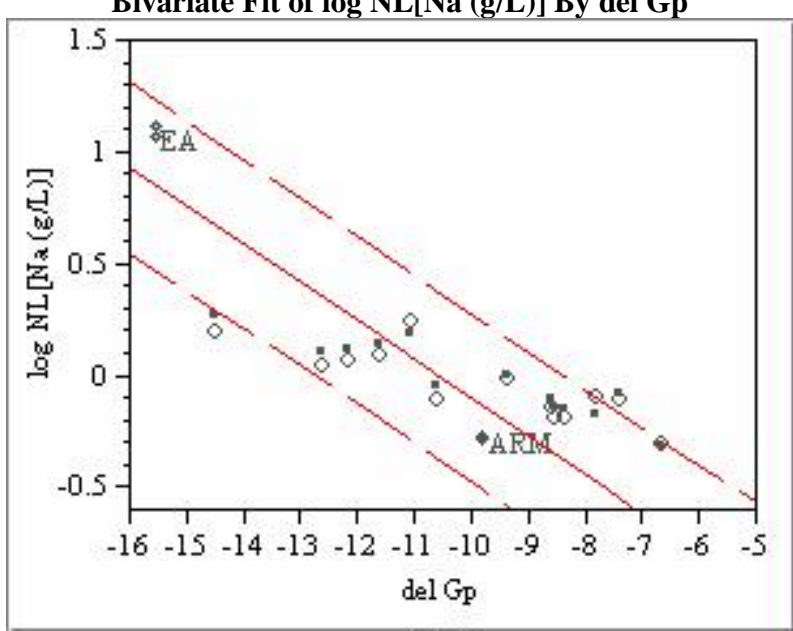

Bivariate Fit of $\log \mathrm{NL}[\mathrm{Si}(\mathrm{g} / \mathrm{L})]$ By del Gp

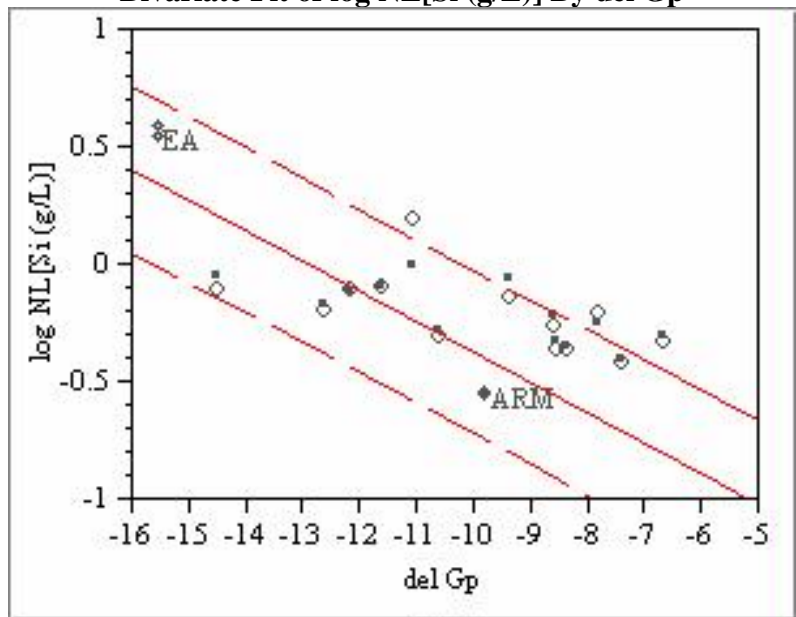


Exhibit E25. PCT vs Del Gp for Underwashed Case; Targeted Compositions

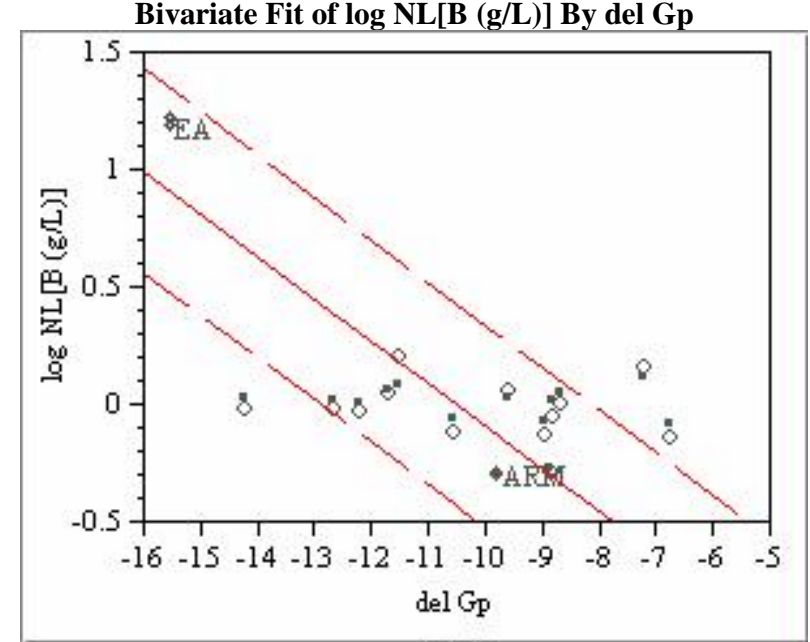

Bivariate Fit of $\log \mathrm{NL}[\mathrm{Li}(\mathrm{g} / \mathrm{L})]$ By del Gp

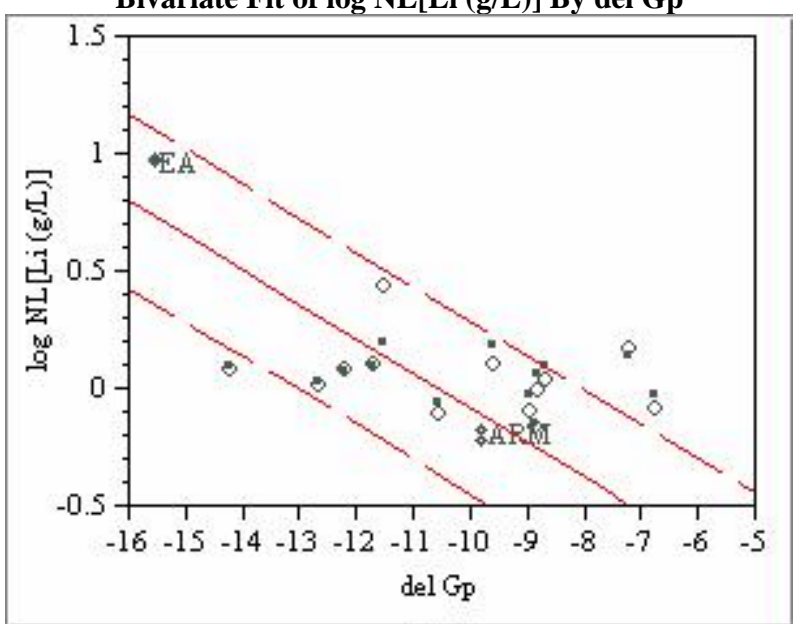

Bivariate Fit of $\log \mathrm{NL}[\mathrm{Na}(\mathrm{g} / \mathrm{L})]$ By del Gp

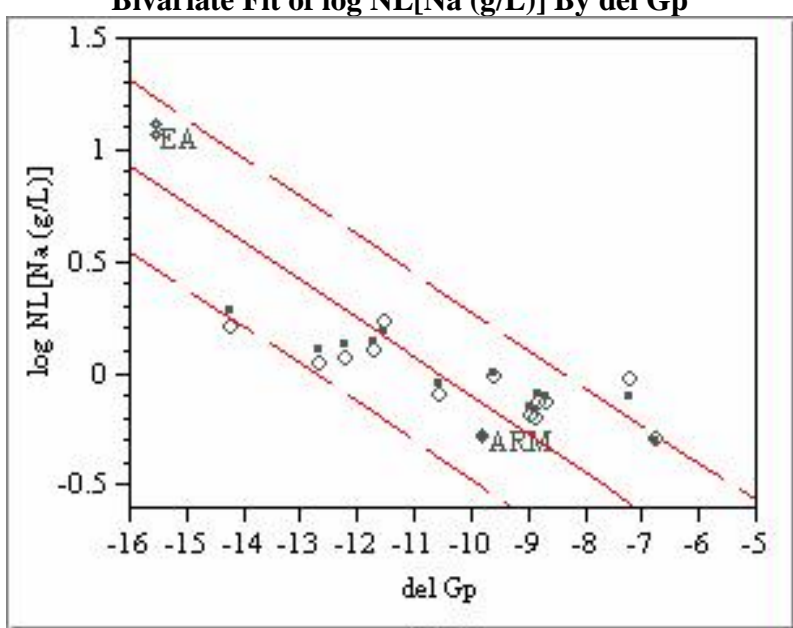

Bivariate Fit of $\log \mathrm{NL}[\mathrm{Si}(\mathrm{g} / \mathrm{L})]$ By del Gp

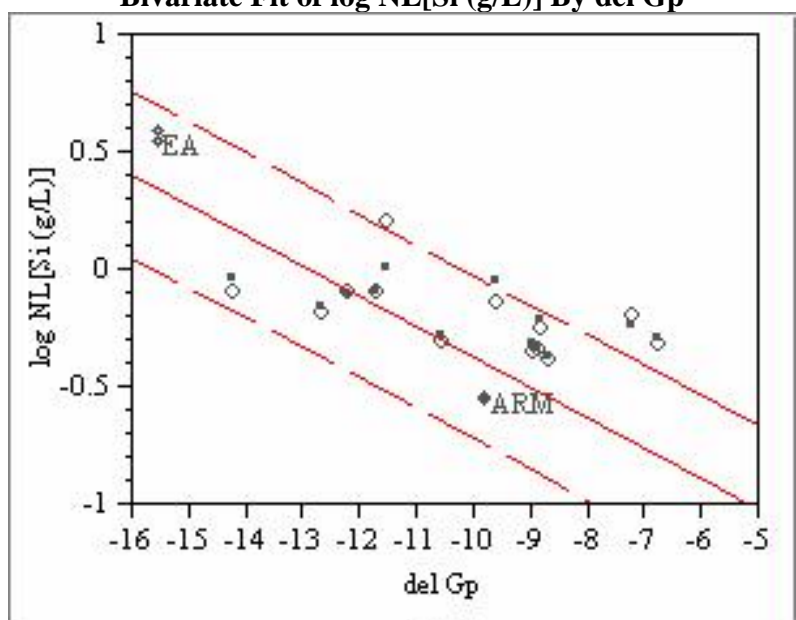




\section{Exhibit E 26. Q uenched versus Centerline Cooled PCTs For N ominally-Washed Case}

Difference:

$\log \mathrm{NL}[\mathrm{B}(\mathrm{g} / \mathrm{L})]$ quenched-log $\mathrm{NL}[\mathrm{B}(\mathrm{g} / \mathrm{L})]$ clc

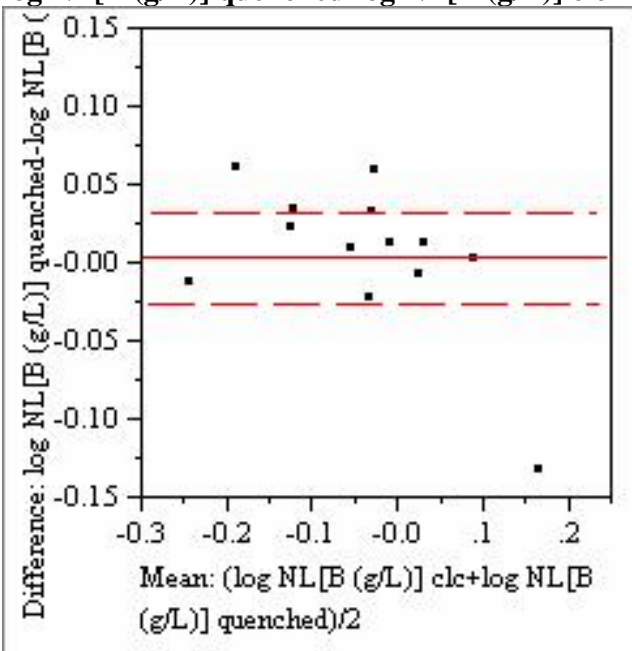

\begin{tabular}{lrlr}
\hline $\log$ NL[B $(\mathrm{g} / \mathrm{L})]$ quenched & -0.0377 & $\mathrm{t}-$ Ratio & 0.273934 \\
$\log$ NL[B $(\mathrm{g} / \mathrm{L})]$ clc & -0.0414 & $\mathrm{DF}$ & 12 \\
Mean Difference & 0.0037 & Prob $>|\mathrm{t}|$ & 0.7888 \\
Std Error & 0.0135 & Prob $>\mathrm{t}$ & 0.3944 \\
Upper95\% & 0.03312 & Prob $<\mathrm{t}$ & 0.6056 \\
Lower95\% & -0.0257 & & \\
$\mathrm{~N}$ & 13 & & \\
Correlation & 0.93479 & &
\end{tabular}

Difference:

$\log \mathrm{NL}[\mathrm{Li}(\mathrm{g} / \mathrm{L})]$ quenched-log NL[Li (g/L)] clc

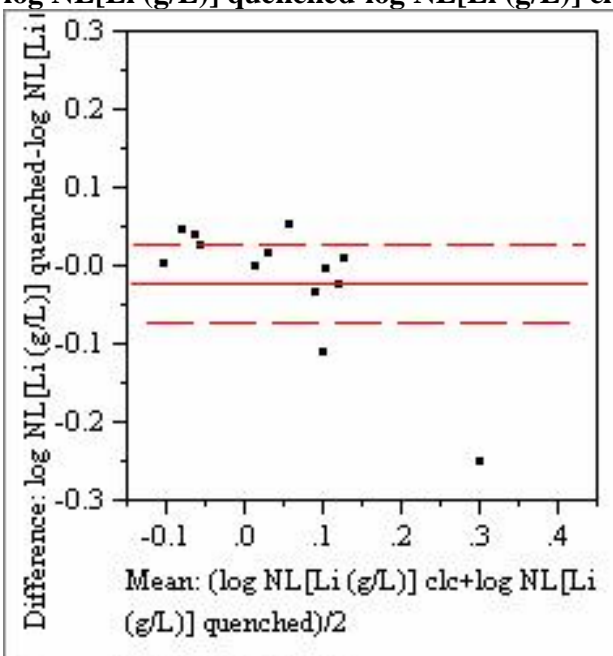

\begin{tabular}{lrlr}
\hline $\log$ NL[Li $(g / L)]$ quenched & 0.04012 & t-Ratio & -0.9532 \\
$\log$ NL $[\mathrm{Li}(\mathrm{g} / \mathrm{L})]$ clc & 0.06188 & DF & 12 \\
Mean Difference & -0.0218 & Prob > |t $\mid$ & 0.3593 \\
Std Error & 0.02282 & Prob > t & 0.8204 \\
Upper95\% & 0.02797 & Prob < t & 0.1796 \\
Lower95\% & -0.0715 & & \\
N & 13 & & \\
Correlation & 0.88012 & &
\end{tabular}

Difference:

$\log \mathrm{NL}[\mathrm{Na}(\mathrm{g} / \mathrm{L})]$ quenched-log $\mathrm{NL}[\mathrm{Na}(\mathrm{g} / \mathrm{L})]$ clc

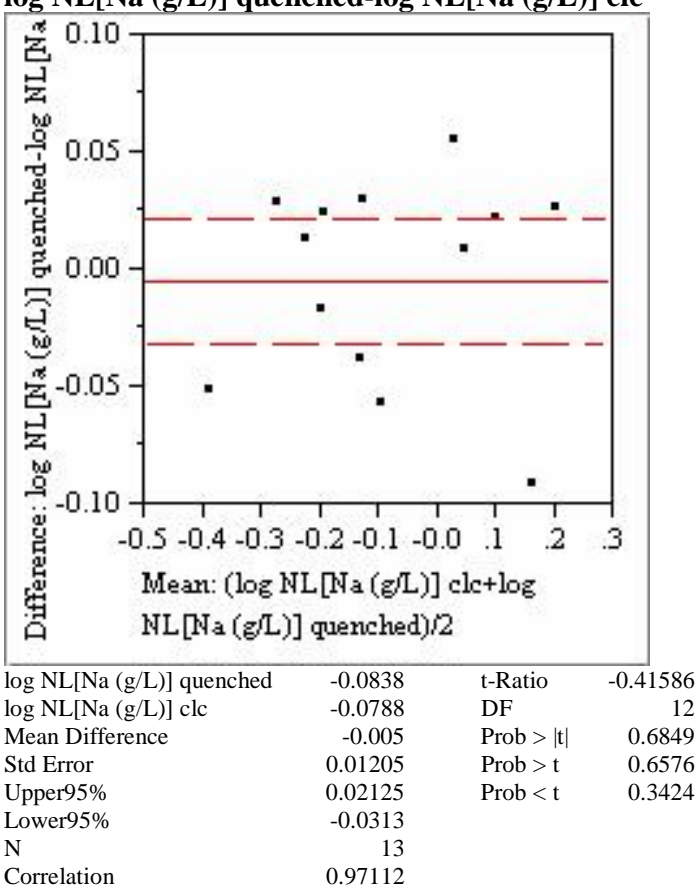

Difference:

$\log \mathrm{NL}[\mathrm{Si}(\mathrm{g} / \mathrm{L})]$ quenched-log NL[Si $(\mathrm{g} / \mathrm{L})]$ clc

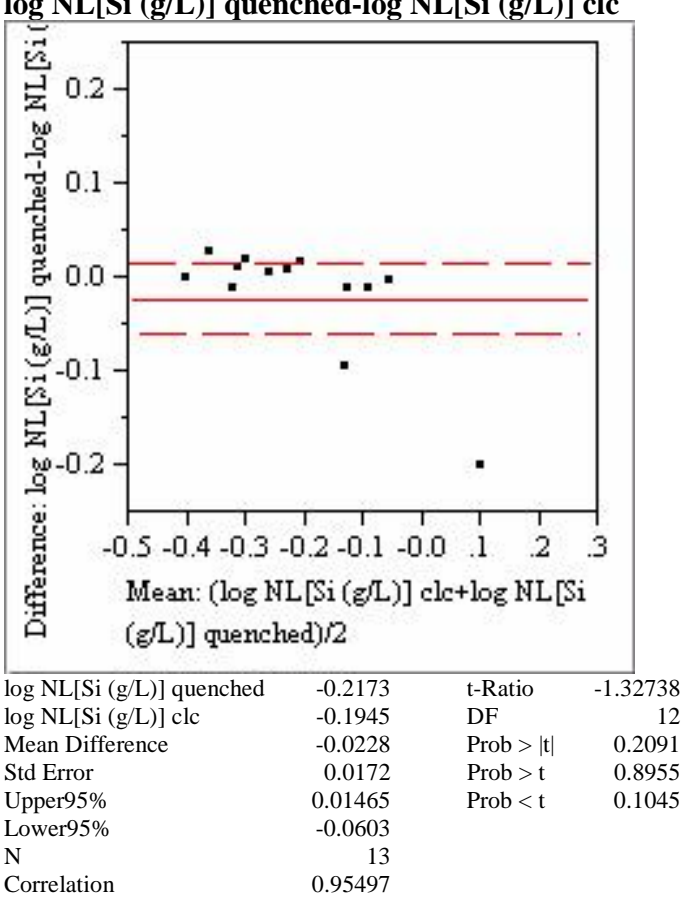




\section{Exhibit E 27. Quenched versus Centerline Cooled PCTs For Underwashed Case}

Difference:

$\log N \mathrm{~L}[\mathrm{~B}(\mathrm{~g} / \mathrm{L})]$ quenched-log $\mathrm{NL}[\mathrm{B}(\mathrm{g} / \mathrm{L})]$ clc

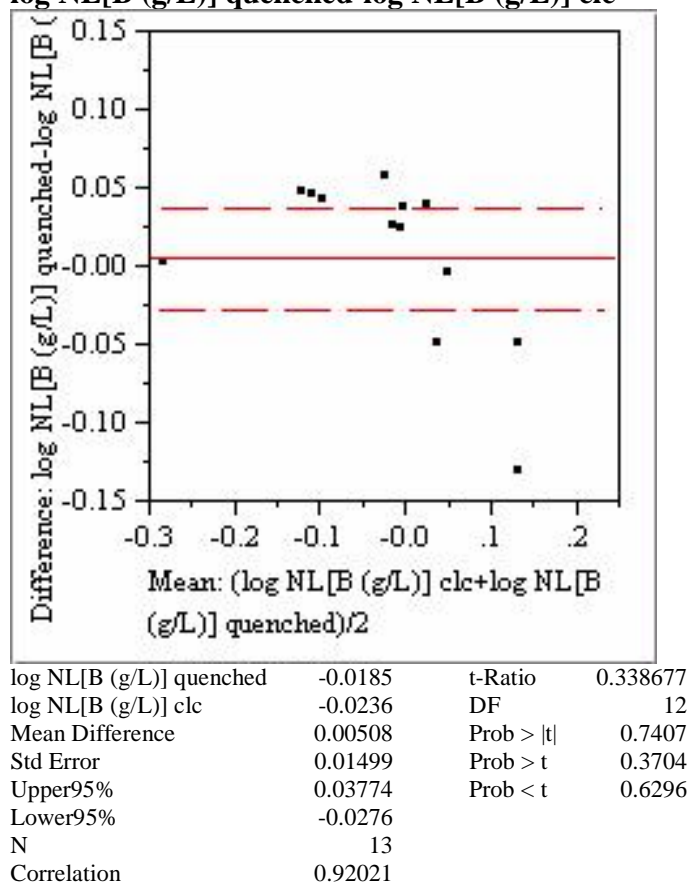

Difference:

$\log \mathrm{NL}[\mathrm{Li}(\mathrm{g} / \mathrm{L})]$ quenched-log $\mathrm{NL}[\mathrm{Li}(\mathrm{g} / \mathrm{L})]$ clc

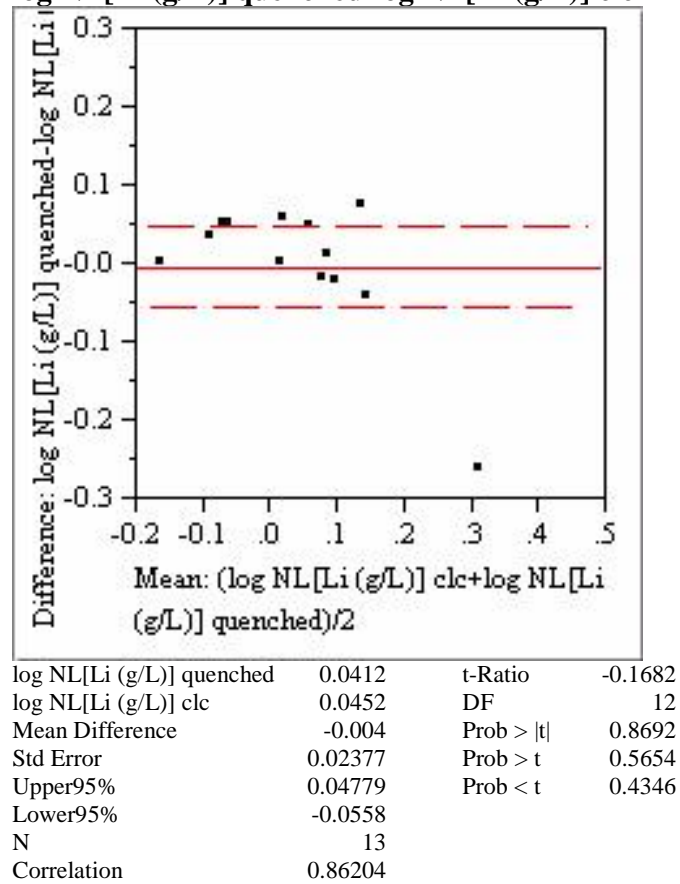

Difference:

$\log \mathrm{NL}[\mathrm{Na}(\mathrm{g} / \mathrm{L})]$ quenched-log $\mathrm{NL}[\mathrm{Na}(\mathrm{g} / \mathrm{L})]$ clc

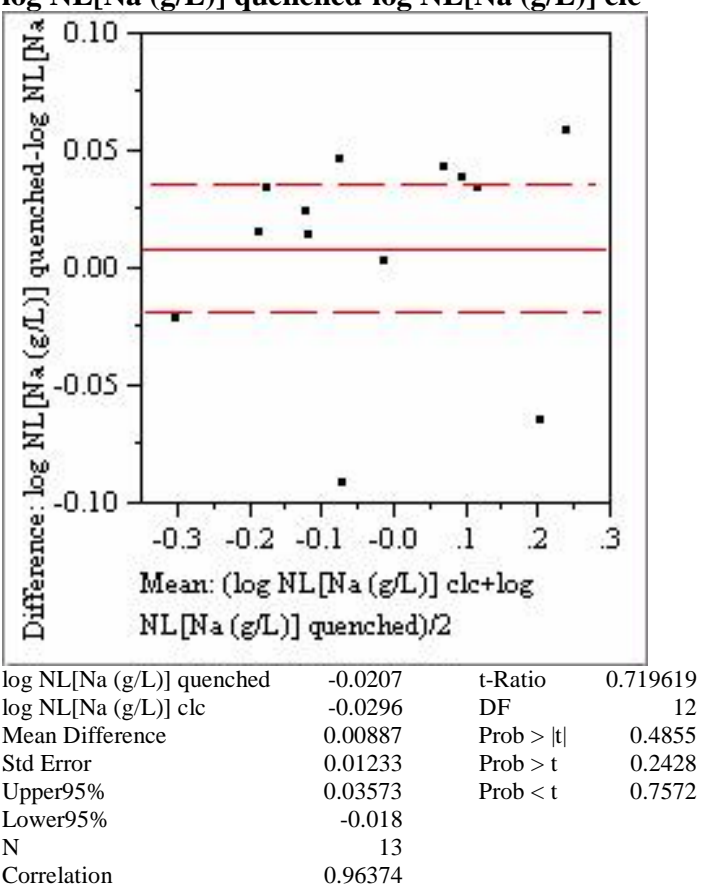

Difference:

$\log \mathrm{NL}[\mathrm{Si}(\mathrm{g} / \mathrm{L})]$ quenched-log NL[Si (g/L)] clc

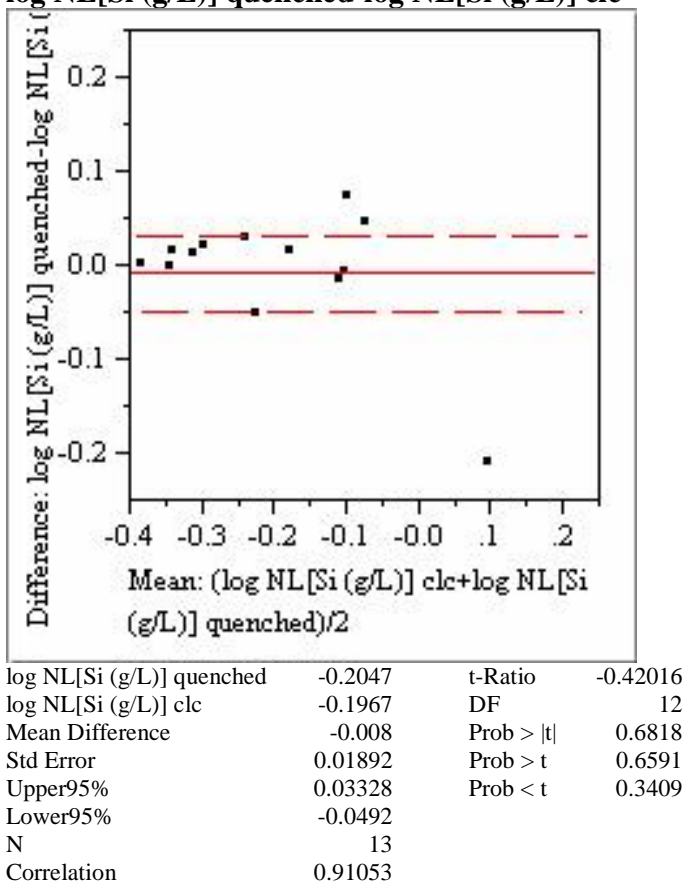




\section{Distribution}

W. D. Kerley, 704-S

D. C. Witt, 704-1T

J. F. Ortaldo, 704-S

M. E. Stone, 704-1T

R. E. Edwards, 704-3N

D. P. Lambert, 704-1T

M. R. Norton, 704-27S

M. F. Williams, 704-1T

J. E. Occhipinti, 704-27S

D. C. Koopman, 704-1T

J. F. Sproull, 704-30S

T. K. Snyder, 773-42A

D. C. Iverson, 704-30S

J. J. Connelly, 773-41A

R. J. O'Driscoll, 704-30S

K. G. Brown, 704-1T

L. M. Papouchado, 773-A

D. R. Best, 773-41A

E. W. Holtzscheiter, 773-A

D.K. Peeler, 773-43A

R. H. Spires, 773-A

T.H. Lorier, 773-23A

D. A. Crowley, 773-43A

D. H. Miller, 786-1A

S. L. Marra, 704-1T

T. B. Edwards, 773-42A

D. F. Bickford, 773-43A

C. M. Jantzen, 773-A

J.C. George, 773-43A

Records (4)

VT QA File 